NBS MONOGRAPH 99

\title{
Automatic Typographic-Quality
}

Typesetting Techniques:

A State-of-the-Art Review

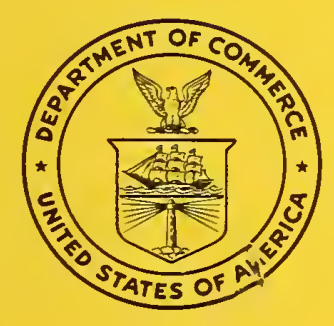




\section{THE NATIONAL BUREAU OF STANDARDS}

The National Bureau of Standards ${ }^{1}$ provides measurement and technical information services essential to the efficiency and effectiveness of the work of the Nation's scientists and engineers. The Bureau serves also as a focal point in the Federal Government for assuring maximum application of the physical and engineering sciences to the advancement of technology in industry and commerce. To accomplish this mission, the Bureau is organized into three institutes covering broad program areas of research and services:

THE INSTITUTE FOR BASIC STANDARDS ... provides the central basis within the United States for a complete and consistent system of physical measurements, coordinates that system with the measurement systems of other nations, and furnishes essential services leading to accurate and uniform physical measurements throughout the Nation's scientific community, industry, and commerce. This Institute comprises a series of divisions, each serving a classical subject matter area:

-Applied Mathematics-Electricity-Metrology-Mechanics-Heat-Atomic PhysicsPhysical Chemistry-Radiation Physics-Laboratory Astrophysics ${ }^{2}$ - Radio Standards Laboratory, ${ }^{2}$ which includes Radio Standards Physics and Radio Standards EngineeringOffice of Standard Reference Data.

THE INSTITUTE FOR MATERIALS RESEARCH ... conducts materials research and provides associated materials services including mainly reference materials and data on the properties of materials. Beyond its direct interest to the Nation's scientists and engineers, this Institute yields services which are essential to the advancement of technology in industry and commerce. This Institute is organized primarily by technical fields:

- Analytical Chemistry-Metallurgy-Reactor Radiations-Polymers-Inorganic Materials-Cryogenics ${ }^{2}$ - Materials Evaluation Laboratory-Office of Standard Reference Materials.

THE INSTITUTE FOR APPLIED TECHNOLOGY ... provides technical services to promote the use of available technology and to facilitate technological innovation in industry and government. The principal elements of this Institute are:

-Building Research-Electronic Instrumentation-Textile and Apparel Technology Center-Technical Analysis-Center for Computer Sciences and Technology-Office of Weights and Measures-Office of Engineering Standards Services--Office of Invention and Innovation-Clearinghouse for Federal Scientific and Technical Information. ${ }^{3}$

\footnotetext{
' Headquarters and Laboratories at Gaithersburg, Maryland, unless otherwise noted; mailing address Washington, D.C. 20234.

${ }^{2}$ Located at Boulder, Colorado 80302.

${ }^{3}$ Located at 5285 Port Royal Road, Springfield, Virginia 22151.
} 
UNITED STATES DEPARTMENT OF COMMERCE - Alexander B. Trowbridge, Acting Secretary NATIONAL BUREAU OF STANDARDS • A. V. Astin, Director

\title{
Automatic Typographic-Quality Typesetting Techniques: A State-of-the-Art Review
}

\author{
Mary Elizabeth Stevens and John L. Little \\ Center for Computer Sciences and Technology \\ Institute for Applied Technology \\ National Bureau of Standards \\ Washington, D.C.
}

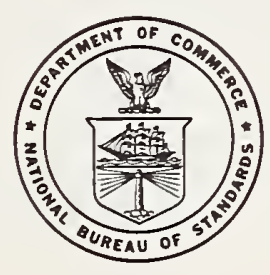

National Bureau of Standards Monograph 99 Issued Aprị 7, 1967 
Library of Congress Catalog Card No. 66-62198 


\section{Foreword}

The Research Information Center and Advisory Service on Information Processing, (RICASIP), which has been incorporated in the Technical Information Exchange, Center for Computer Sciences and Technology, National Bureau of Standards, has been engaged in a continuing program to collect information and maintain current awareness about research and development activities in the field of information processing and retrieval. An important responsibility of RICASIP which has been jointly supported by the National Science Foundation and NBS, has been the preparation of state-of-theart reviews on topics of current interest in various areas of this broad field.

This report is one of a series intended as contributions toward improved interchange of information among those engaged in research and development in this field. The report considers new techniques for typographic-quality composition based upon machine generation, manipulation, and processing of textual and other materials to be reproduced in multiple copies for distribution or publication.

It should be noted that names and descriptions of specific proprietary devices and equipment have been included for the convenience of the reader, but completeness in this respect is recognized to be impossible. It is also to be noted that certain important developments have remained proprietary or have not been reported in the open literature. Some of the major contributors to key developments in the field may thus have been inadvertently omitted.

The omission of any method or device does not necessarily imply that it is considered unsuitable or unsatisfactory. Conversely, inclusion of descriptive material on any proprietary instrument, product, or process does not constitute endorsement. In general, we shall be concerned with the techniques that offer reproduction copy of typographic quality, which may be defined in terms of a variety of f factors involving human engineering, aesthetic, economic, and productivity considerations.

It is to be noted that this report was prepared on the Linofilm equipment of the United States Government Printing Office.

A. V. Astin, Director. 



\section{Contents}

Foreword.....

1. Introduction

1.1 Purpose and scope of report.

1.2 Background.

2. Typographic quality and the compositor functions.

3. Hardware for typographic composition

3.1 Hot metal casting equipment.

3.1.1 Line casting equipment...

3.1.2 Tape-controlled hot metal typesetters...

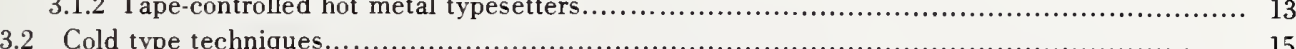

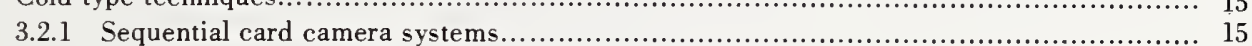

3.2.2 Ribbon impression machines without tape perforators.................................... 17

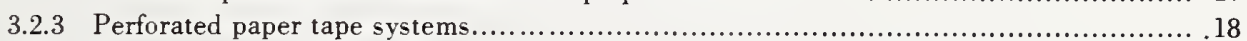

3.2.4 Tape perforators without printers....................................................... 18

3.2.5 Teletypesetter systems............................................................ 20

3.2.6 Tape perforators with ribbon impression printers........................................ 20

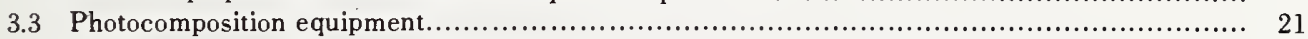

3.3.1 Techniques of photocomposition.................................................... 22

3.3.2 Classes of photocomposers........................................................ 22

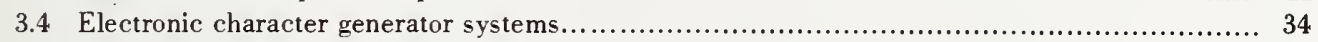

3.4.1 Techniques of electronic character generation........................................ 34

3.4 .2 Specific character generating equipment.............................................. 35

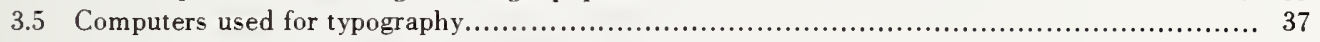

3.5.1 Small special-purpose typography computers...................................... 37

3.5.2 General purpose computers used for typography..................................... 38

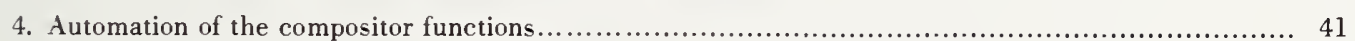

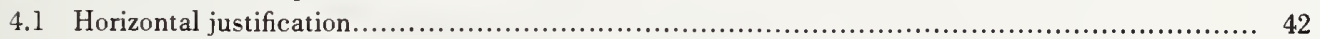

4.1.1 Equipment and techniques for horizontal justification................................. 43

4.1.2 Semi-automatic and automatic hyphenation.......................................... 44

4.2 Corrections, updating, and merging........................................................ 50

4.3 Vertical justification and page composition................................................ 53

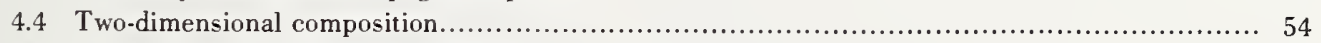

4.5 Specific photocomposing equipment....................................................... 56

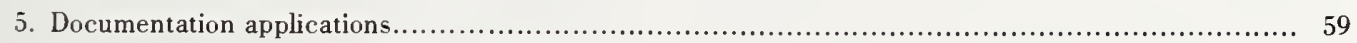

5.1 General purpose computer programs..................................................... 60

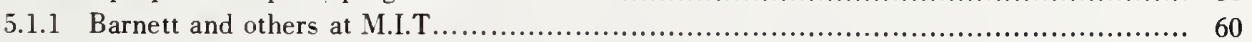

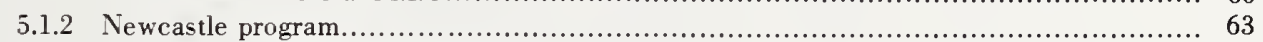

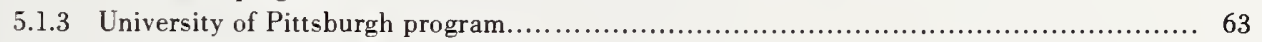

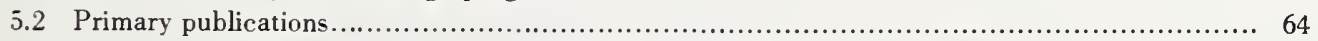

5.2.1 Computer-compiled and computer-generated primary publications....................... 64

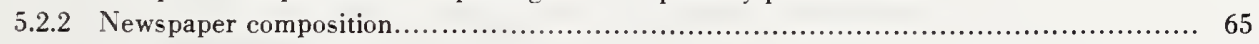

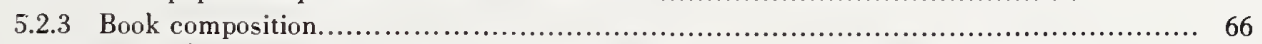

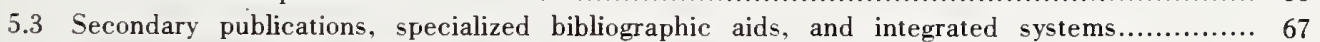

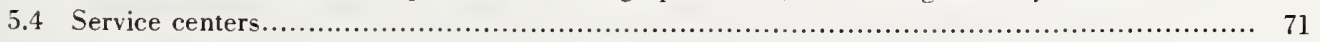

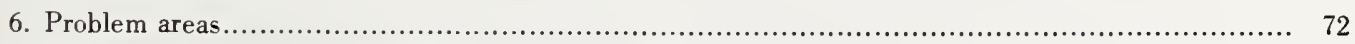

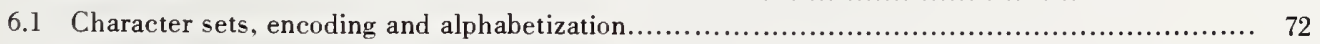

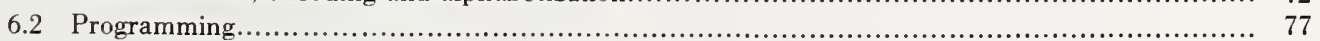

6.3 Implications for management planning. industry adjustment and systems design................ 79

6.3.1 Problems of author-editor-publisher relationships....................................... 79

6.3.2 The possible role of automatic character recognition.................................. 80

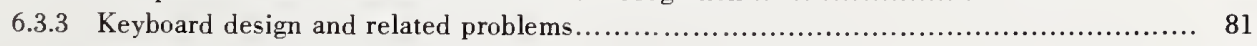

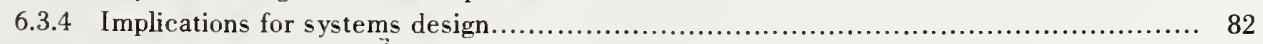

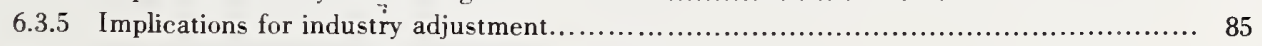

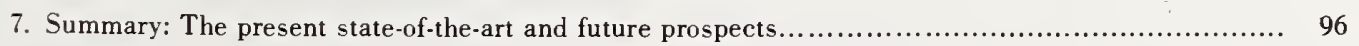

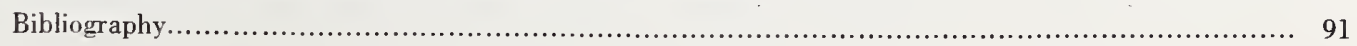




\section{List of Figures and Tables}

Figure 1, Example of computer-typeset tables of physical data........................

Figure 1. Example of computer-typeset tables of physical data...........................................6. 6

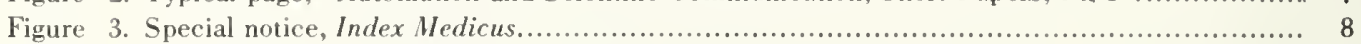

Figure 4a, 4b. Foundry type character matrices and example of cast line........................... 11

Figure 5. Linotype Comet........................................................................... 12

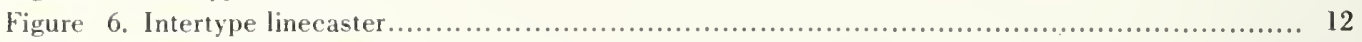

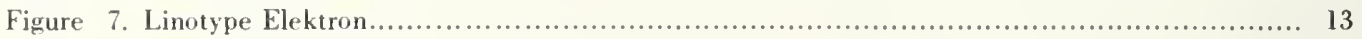

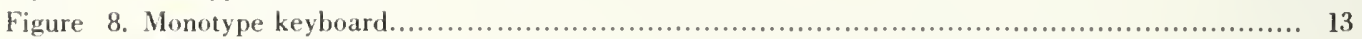

Figurc 9. Monotype caster.................................................................. 14

Figure 10. Monotype matrix case................................................................. 14

Figure 11. Recordak Listomatic ........................................................... 16

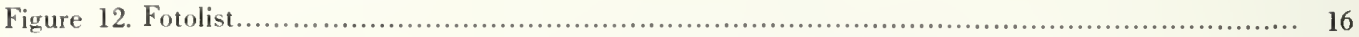

Figure 13. Compos-0-line......................................................................... 16

Figure 14. Fairchild Graphic Standard Teletypesetter perforator.................................. 18

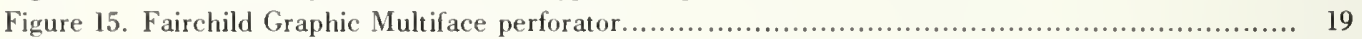

Figure 16. Mergenthaler Linomatic perforator....................................................... 19

Figure 17. ATF Typesetter....................................................................... 19

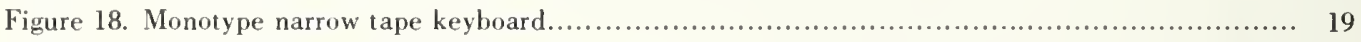

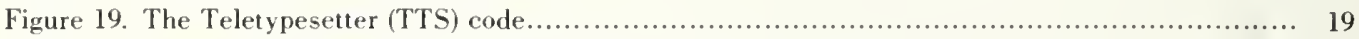

Figure 20. Perforated tapes used in typesetting................................................. 21

Figure 21. Sample output, Varityper Series 800 photocomposing machines.............................. 23

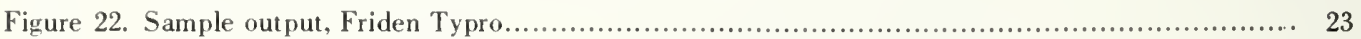

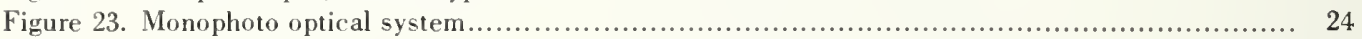

Figure 24. Intertype Fotosetter.................................................................. 25

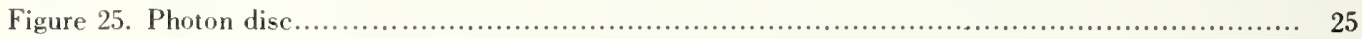

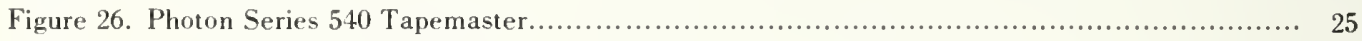

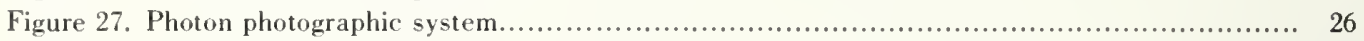

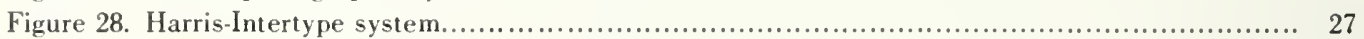

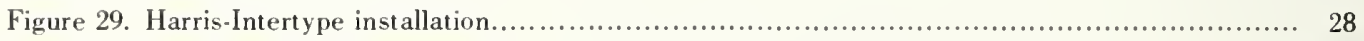

Figure 30. Harris-Interty pe equipment........................................................ 28

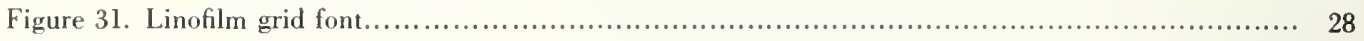

Figure 32. Linofilm grid font turret............................................................. 29

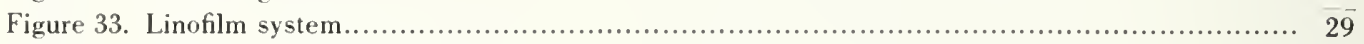

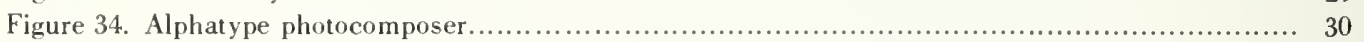

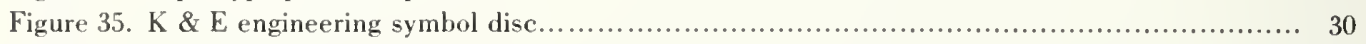

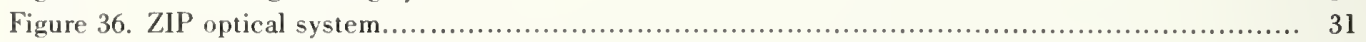

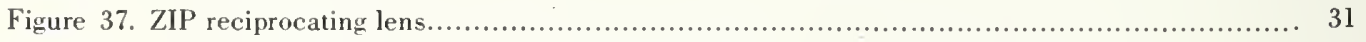

Figure 38. GRACE installation, National Library of Medicine........................................... 32

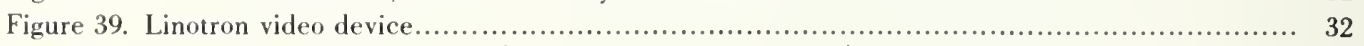

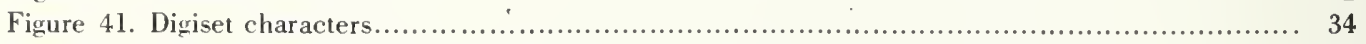

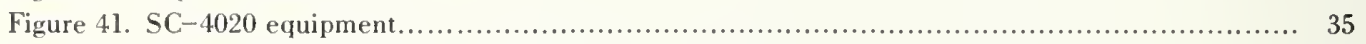

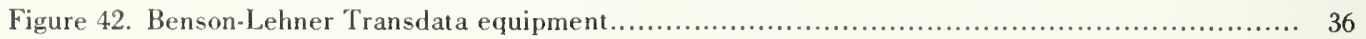

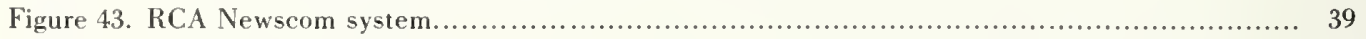

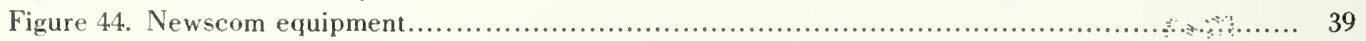

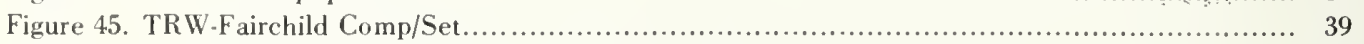

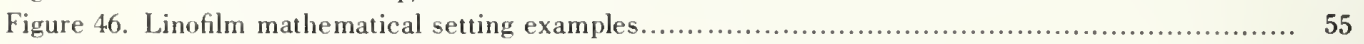

Figure 47. Output of Technical Text Compiler...................................................... 57

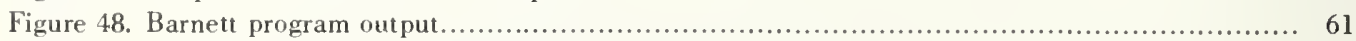

Figure 49. Sample page, Chemical-Biological Activities............................................. 74

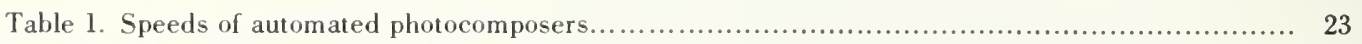

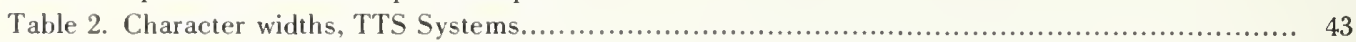

Table 3. Companies represented at a photocomposer bidders conference, Government Printing Office, August 12, 1963 . 


\title{
Automatic Typographic-Quality Typesetting Techniques: A State-of-the-Art Review
}

\author{
Mary Elizabeth Stevens and John L. Little
}

\begin{abstract}
This report describes the current state-of-the-art in automation of graphic arts composition, starting from either of two sources: (1) keyboard entry of manuscript material, or (2) mechanized input in the form of available perforated tapes or magnetic tapes. The gamut is covered from one extreme in which a skilled keyboard operator performs all of the compositor functions required to operate a typesetting machine, to the other extreme in which the input merely provides text whether or not including designation of desired font changes, followed by a high degree of automation through all operations leading to type set for printing. Intermediate automation aids for the compositor functions, including characteristics of special-purpose digital computers and functions performed by typography programs for general-purpose digital computers, are reviewed.

Characteristics of automatically operated typesetting mechanisms, including hot metal casting machines and photocomposers, slow, medium and high speed, are outlined. Applications of new techniques for typographic-quality automated composition that are of interest in scientific and technical information centers, libraries and other documentation operations include sequential card camera listings, computer-generated KWIC indexes, photocomposition of technical journals, automatic composition of books containing both computer-produced tabular data and natural language texts, and the incorporation of mechanized processes throughout the publication cycle from the author's original manuscript preparation to the final printing. A bibliography of 363 references is included.
\end{abstract}

Key words: Automated type composition, computerized typesetting, graphic arts, photocomposition, printing and publication.

\section{Introduction}

\subsection{Purpose and Scope of Report}

This report is one of a series prepared for the Research Information Center and Advisory Service on Information Processing (RICASIP), and intended as a contribution to improved cooperation in the fields of information selection systems development, information retrieval research and mechanized translation. In each of these areas, increased use of automation has led to increased concern both for high speed and for high quality of machine output.

Methods range from manual keyboard entry of textual material to computer printouts at high speed but low quality (uppercase, fixed spacing and poor paper utilization). There is a third class of methods involving machine and computer-generated tapes to control automatic typesetting to batteries of low speed composing-printing devices. Finally there is a new class involving computer control via magnetic tape of high-speed composition systems. At one extreme, a skilled keyboard operator performs all of the compositor functions required to operate a typesetting machine; at the other, the input merely provides text copy, which may or may not include designations of desired font, style, size, and format changes, to be processed with a high degree of automation through all operations necessary to produce type or plates set for printing.
The practical realization of several major technological developments affecting both printing and data processing operations has so recently been achieved that a state-of-the-art report on this topic may appear to be premature. Nevertheless, with the initiation of planning for either high-speed photocomposition or direct computer output of typographic quality, or both, these developments herald wide-spread adoption in many areas of documentation. There is, in addition, considerable advantage to be gained in systems planning and inter-system cooperation if users not now contemplating application in their own operations are nevertheless familiar with such applications as those of STAR (Scientific and Technical $A$ erospace Reports of the "National Aeronautics and Space Administration) and MEDLARS (Medical Literature $A$ nalysis and Retrieval System of the National Library of Medicine) and with the general potentialities of these new techniques.

Anticipating the probable impact of these developments upon many organizations active in the handling of scientific and technical information, RICASIP in 1962 contracted with Information Dynamics, Inc., for a review of the situation. The present report is based in part on this earlier study. Where appropriate, portions of the text of the IDC report prepared by Lawrence $\mathrm{H}$. Berul have been incorporated in this report. 
The Information Dynamics report to RICASIP was followed by an updated and condensed version prepared for the conference on "Libraries and Automation" held at Airlie House in May of 1963, (Sparks et al., 1964)." Since the later version incorporates much of the material originally prepared for RICASIP, it will also be quoted extensively as appropriate.

Where exceptionally good reviews about a particular system or piece of equipment have been published earlier, these will be referenced and only brief mention will be given in this report. In particular, nearly all of the pioneering exponents of either photocomposition or computer-driven composition techniques (whether linecasting or film setting or both in combination) have published at least brief accounts of their efforts and proposals. In alphabetical order, for illustrative example, we note: Bafour, Barnett, Bozman, Duncan, the two IBM-Mergenthaler teams, Kuney-American Chemical Society, Luhn, the Medlars system, Moyroud, the NASA system, and others.

In a supplement to the November 1964 edition of Print in Britain, there was set out a check list of machines, materials, and methods for both filmsetting and cold type methods which was characterized by Robert E. Rossell, Managing Director of the Research and Engineering Council of the Graphic Arts Industry, Inc., in a letter covering distribution of reprints to members of the council, as "the most comprehensive checklist of machines, materials, and methods on the subject that we have uncovered to date."

This report attempts to describe the principal automation techniques and the ingenious interweaving of these techniques into a multitude of configurations each attempting in its own particular manner to accomplish the compositor functions by mechanized aids to human effort. Because the report is one of a series intended as contributions toward inproved interchange of information among those engaged in research and development in the fields of information processing, information selection, and information retrieval, the applications stressed are generally in the area of scientific and technical information handling and related documentation operations.

Applications of these techniques that are of interest in scientific and technical information centers, abstracting and indexing services, publishing houses, libraries, and other documentation operations include sequential card camera listings of bibliographic information, computer-generated Key $W$ ord-in-Context (KWIC) indexes, photo-composition of technical journals, and announcement bulletins, automatic composition of books or reports containing both computer-produced tabular data and natural language text, and the incorporation of mechanized processes throughout the pub-

Note: Bibliographic citations to references are arranged in alphabetical order by author or title in the appendix, pp. $91-98$. lication cycle from the author's original preparation of manuscript to the final printing.

The emphasis will be on devices and techniques which provide typographic quality in the outputproportional spacing, upper and lower case, large character sets, variety of fonts and type sizes, margin and column justification, and the like. Generally, the definitions of typographic quality are subjective and not objective. That is, while type designers and typographers have followed precise traditions of craftsmanship through the centuries, the bases for their specifications have been aesthetic or artistic rather than scientific findings as to legibility or readability.

\subsection{Background}

The use of machines and of mechanizable processes to improve the utilization of scientific and technical information has a first and obvious area of application in the preparation of copies of text and tabular data for distribution or publication. Mechanized printing began with the invention of movable type as applied to this purpose since the 15th century, the first scientific journal began publication in $1665,{ }^{2}$ the first abstract journal appeared in $1807 .^{3}$

Today, the increasing demands for timeliness and economy of both primary and secondary publications and the emergence of the computer itself as the generator of data tabulations, composite test report compilations, extracted portions of prestored text, index and citation listings (and even automatically prepared "abstracts" or translations) impose new requirements for speed and costeffectiveness of production and for customer acceptance of the machine-prepared products.

A special problem has been that of currency of coverage and prompt dissemination by secondary publications (catalogues of holdings, announcement of acquisition bulletins, index lists or abstract journals, and cumulative indexes to all of these). In particular, the question of revision and updating of existing catalogs and files, for re-issue, has been an important factor in many of the existing deficiencies of both current awareness and retrospective search systems.

The importance of improved techniques of typesetting and composition to the whole area of generating, processing, disseminating and using scientific and technical information manifests itself in at least three major ways. First, improved techniques offer great promise of speed and increased economy in the editing, preparation for printing, and proofreading of primary publications. Secondly, they offer new potentialities of economy and timeliness in the preparation of announcement 
bulletins, indexes, abstract journals, printed catalogues, demand bibliographies, and other vital aids to maintenance of current awareness and to literature search. Finally, they offer the prospects of restoring greater legibility and readability to materials compiled or produced by computer, thus enhancing the utility of machine output, again offering significant proofreading advantages for computed material such as extensive numerical tables.

The art of printing, originating with the Chinese who made wood engravings for printing on silk, has an interesting history of development. About the 12 th century, B.C., the Chinese first introduced wooden blocks of movable type. The development of this technique which led to modern printing is generally attributed to Johannes Gutenberg, a German stonemason, who in the decade of 1440 demonstrated the practical use of movable metal type.

Production of printed pages thus dates back at least 500 years. Gradual mechanization of typesetting operations occurred during the following four centuries, including the introduction of keyboards for type selection and the development of devices for the automatic sorting of used type slugs. An interesting early example of possible mechanical processing for composition of revised and updated bibliographic listings was reported by Charles C. Jewett to a convention of librarians held in New York in 1853.

"The title of every book and of each distinct edition is stereotyped upon a separate plate. The author's name also stands by itself. Each plate shows at a glance the heading to which it belongs. It is obvious that these plates may be placed together in alphabetical order, as may be desired. They are mounted on blocks, for printing like other stereotype plates. It is proposed to preserve the plates or blocks in alphabetical order of the titles, so as to be able readily to insert additional titles, in their proper places, and then to reprint the whole catalogue. By these means, the chief cost of republication (that of composition) together with the trouble of revision and correction of the press. would, except for new titles, be avoided." 4

Jewett went on to claim such advantages as the following: ". . . The great ends to be gained are:

1. To avoid the necessity of preparing, composing, and correcting anew the titles once printed, when the Library has received acces. sions, or the alternative of printing the titles of these accessions in supplements, which are very inconvenient appendages.

2. To prevent the repetition of the work of preparation of titles, composition and correction of press, for copies of the same book in different libraries. The title once prepared and stereotyped remains at the Smithsonian Institution, to be used by any library having the same book.

3. To secure uniformity in the construction of catalogues, thus greatly facilitating the researches of the student . .

"There will also be great saving of the expense of paper and presswork under this system. It is customary now to print off a larger number of copies of every catalogue than are immediately wanted, because it cannot be known how many may be required before the catalogue may be reprinted. On this plan. when a new edition, with all additions incorporated, can be had at any time, it will not be thought necessary to print more copies than enough to meet the immediate demand." 5

As Clapp reports, "a sample catalog, printed in 1854 for the Library of Congress from these plates, still exists and constitutes one of the handsomest pieces of printing produced in the 19th century. Unfortunately, however, Jewett was betrayed by the imperfect technology of the time. The plates warped in storage; the information was lost and with it the success of the venture." ${ }^{6}$ Not until nearly a century later, with both manual and sequential card camera shingling techniques, were some of these advantages realized.

In the late 19th century, a great leap forward had been made in typographic composition by the invention of two ingenious and powerful machinesthe Linotype by Mergenthaler and the Monotype by Lanston. The Linotype, first used by the New York Herald Tribune in the late 1800's, is a keyboard-operated machine which casts a hot metal slug for each complete line of type. The Monotype equipment involves two separate machines. First, a large keyboard is used to operate a paper tape perforator. The 31 channels of this tape store information with respect to designated characters, fonts, type widths, and space widths. This perforated paper tape then controls the operation of the second machine, a hot metal type slug caster, which casts each symbol on a separate type slug and which uses controlled dimensioning to fill out a complete line by insertion of additional type slugs to provide the required spacing.

Thus, equipment to accomplish in standing type the full range of functions covered by this report, from keyboard, perforated tape and typesetting for printing, was already available in the 19th century. However, the complete range of compositor functions was then concentrated in the keyboard operator, who needed to be a highly skilled person with a production capability sharply limited by the necessity to keyboard all composition details, and who was restricted especially by the requirements for manual page make-up.

During the last few decades, and particularly during the most recent decade, there has been a notable increase in the availability of mechanical aids in composition operations. Chronologically, some of the key developments have been as follows: 
The first patent applications for what is now known as the Photon (the Lumitype in France) were filed in 1945. Since that time the inventors, Messrs. Higonnet and Moyroud, have taken out over 40 U.S. patents as well as numerous foreign patents on various inventions in the field of photocomposition. Further impetus was given to this type of photocomposition technology by the Graphic Arts Research Foundation Inc., from about 19481949 onward (Higonnet and Moyroud, 1957, 1960).

In 1946, Corrado reports, "Mergenthaler was experimenting with a cathode ray tube, closed loop, television system to produce type on film. The state-of-the-art of cathode ray tubes was such that the tubes did not have the resolving power or the illumination capability to produce the quality of type that most people wanted . . "7 Instead of pursuing this approach further, therefore, work was begun on the photocomposer Linofilm, the first prototype production model having been tested in the period 1955-1956.

At about this time, or earlier, the Intertype Corporation also was experimenting on phototypesetting techniques in cooperation with the Govern. ment Printing Office, leading to development of the Fotosetter.

Eastman Kodak was active in the field of electronic character generation in the middle-to-late 1940 's, leading to demonstrations of DACOM (Datascope $C$ omputer $O$ utput $M$ icrofilmer) equipment in in the early 1950's. As early as 1948, this company filed patent applications with respect to means for displaying characters on the face of a cathode ray tube, using monoscope generating techniques similar to the test pattern techniques used in television systems. These patents were then cross-licensed to RCA, to IBM and to at least one British organization.

Reporting in 1959 on then current progress in photocomposition, Winkler said: "The Fotosetter, developed in 1947 by the Intertype Corporation, was the first commercially available phototypesetting machine. The Photon, of Photon, Inc., was the second phototypesetter placed on the market. In succession, the Monophoto of the Lanston Monotype Company and the Linofilm machine of the Mergenthaler Linotype Company were introduced. The American Type Founders, Inc., has recently made the ATF Typesetter available." 8

Winkler also describes the Research \& Engineering Council of the Graphic Arts Industry, Inc., which was started informally in 1948, and formally incorporated in $19500^{9}$

A special case with respect to automatic aids to type composition, relating to problems of twodimensional or graphic material composition, is that of machine composing of Chinese ideographs. The University of Pittsburgh reports, for example,

\footnotetext{
${ }^{7}$ Corrado, 1964, p. 109

8 Winkler, 1959 , p. 70.

Ibid.. pp. 75-76.
}

that "in 1953-1954 a study of the Chinese language to determine the feasibility of developing a machine that would economically and effectively compose Chinese ideographs in the field was begun." 10

The year 1954, however, marks the official date of disclosure of the first comprehensive approach to the use of modern machine techniques to typographic quality composition generally involving both computer processing and automatically controlled composition devices (Bafour, 1965).

As Duncan reports: "The proposal to apply the power of modern digital computers to text processing originated with Bafour, Blanchard, and Raymond. Their patent application was made in France in March 1954, and must have been preceded by a great deal of work. Their system as originally conceived embodied a special-purpose tape-typewriter keyboard (at least one prototype was built by Barriquand and Marre) with additional keys for function codes, and means for producing correction tapes and merging them before processing in a special-purpose computer, details of whic's were also specified in the patent ... They clearly envisaged that the output would control both automatically operated linecasters and other more advanced photo-composing machines." 11

The year 1958 saw the first publicly available production of KWIC ( $K$ ey $W$ ord-in- $C$ ontext) indexes, those for the Preprints of the International Conference on Scientific Information (machine-produced but not of typographic quality), ${ }^{12}$ the initiation of projects to explore the potentialities of photocomposition at the American Chemical Society ${ }^{13}$ and, in France, "experiments ... carried out using a computer of the Societe d'Electronique et d'Automatisme. The output tapes were fed into the Intertype casters of the Imprimere Nationale, equipped with Fairchild readers and control units." 14

Duncan comments with respect to the latter experiments as follows: "The tragedy is that after the brilliant demonstration of November 1958, the French Syndicate did nothing to exploit the system. The latest information from France is that a large computer firm (SCF) and the typewriter firm of Japy together with Barriquand and Marre are about to carry on the work, having lost a lead of six to ten years." 15

The lead thus lost in France was to be picked up both in the United States and in Great Britain in the period 1959-1962 with respect to photocomposition equipment as such and also with respect to computer control of various compositor functions. Hoffman, in a 1959 survey of composing room materials and machines, reported as follows: "Photog-

\footnotetext{
${ }^{10}$ University of Pittsburgh. 1964, p. 1.

${ }^{11}$ Duncan, 1964, p. 152.

12 Citron, Hart, and Ohiman. 1958, Luhn, 1959

13 Kuney, 1963, p. 249; Skolnick, 1962, p. i.

${ }^{14}$ Bafour, 1965, p. 194

${ }^{15}$ Duncan, 1964, p. 152.
} 
raphy.... is an industrial phenomenon creating a major upheaval in the graphic arts industry. . . . Electronic control of the photographic composing process is already here . . While not at present available commercially, machines such as Monophoto and ATF Typesetter indicate that photographic composition in justified lines of straight matter can be expected soon from several machines." 16

From 1959 to 1961, an investigation and study of "graphic-semantic" composing techniques was conducted by the Syracuse University Research Institute for the Rome Air Development Center (Buck et al.. 1961), which concluded that suitable techniques were available or could be provided under short-range development for good quality page composition combining machine-translated text with original graphic material. IBNl, which was developing the concurrent machine translation efforts, subsequently demonstrated output of text via computer program and Linofilm. Then, the Mergenthaler company conducted a further study of the feasibility of the proposed combined lexicalgraphical composition system.

By 1961. developments at IBM under Rome Air Development Center sponsorship led to the driving of a Linofilm from computer output of translated Russian text. ${ }^{17}$ Thus, "when a translated magnetic tape has been prepared. it is used as input to a 704 digital computer program ... [that] removes all characters which are meant only for the flexo writer. It computes justification information as well as information needed to center a line, or to print a line on the right hand side of the page. It converts the flexowriter codes into the code needed by the Linofilm photo unit. It also converts the edit information which will change the character fonts, the point size and the distance between successive lines." 18

Appropriately, a part of the final report on Mergenthaler's study for RADC of lexical-graphical composer-printer systems design (1963) was itself automatically typeset by computer-Linofilm combination, using the Autoset computer program developed by Mitchell Saxon.

A two-year program for the investigation of the use of photocomposition techniques in the setting of mathematical text, such as the journal Mathematical Revieus, was initiated at the American Mathematical Society in January 1962.

In 1960, as a result of the American Chemical Society's graphic arts research program, production of the quarterly Journal of Chemical and Engineering Data by photocomposition techniques was begun, utilizing manually keyboarded Photon equipment. In the project summary prepared for Current Research and Development in Scientific Documentation, No. 7 (for November 1960). it is stated that results with respect to the economics of the process were not conclusive as of that time, but "there are

\footnotetext{
${ }_{18}$ Hoffman, 1959 , p. 194

17 See Blondeau, 1963, p. 84.

is International Business Machines Corp., "Graphic Composing Techniques", 1962, p. 1 .
}

indications that the process is at the very least competitive with hot metal costs." 19

Concurrently, at M.I.T., Barnett and his associates were engaged in work relating to "the photocomposition of the output of one type of computer (the IBIl 709), on one type of photocomposing unit (the Photon), which is used essentially as a papertape driven off-line printing device." 20 Their early efforts aroused such comments as the following:

"Exciting and promising is the work of Professor 11. P. Barnett at MIT on the use of computers. He has successfully programmed a computer to punch and correct a tape suitable for operating Photon equipment. These are the beginnings of what is visualized as a computer-photocomposition set-up for producing a quality product suitable for reproduction of scientific papers." 21

"As a by-product of their computerized research in theoretical chemistry, these two professors [Barnett and Kelley, MIIT. July 1961] unknowingly had lit one of the major fuses that subsequently erupted into this new era in typesetting technology." 22

For example, as of late 1961. plans were already well under way to use the M.I.T. computer-Photon combination "to produce a Union List of Communist Chinese Serials, under the direction of Ryburn M. Moss, Associate Director of Libraries for M.I.T." ${ }_{23}$ In a 1963 report, presented at the Spring Joint Computer Conference, Barnett et al. provide as Appendix Vill a part of this listing.

The period 1961-1962 was also marked by Mlitra's re-programming of the Bafour-Blanchard-Raymond hyphenation rules for the English language to be processed on Ferranti computers, ${ }^{24}$ by the American Chemical Society's use of the Photon for the Journal of Chemical and Engineering Data and for the production of the Journal of Chemical Documentation beginning with its first issue (January, 1962), ${ }^{25}$ and by the installation by the Compugraphic Corporation of the first Directory Tape Processor at the General Telephone Directory Company on Mlay 1, $1961 .^{26}$

In 1962, the Joint Committee on Printing of the U.S. Congress initiated action which led to the establishment of the Federal Electronic Printing Committee, to the setting up of the post of Electronic Printing Officer at the U.S. Government Printing Office and eventually to the ordering of Linotron equipment for the Government Printing Office. (Nlergenthaler had already entered into a contractual arrangement with the Columbia Broadcasting System Laboratories for cooperation in the graphic arts field, resulting in the eventual Linotron design. $)^{27}$

\footnotetext{
19 Vational Science Foundation, 1960, p. 3.

20 Barnett et al., 1962, p. 58.

"Skolnick, 1962, p. i.

23 Markus, 1962, p. 18; see Shih and Snyder, 1963.

*4 Duncan, 1964, p. 153.

${ }^{25}$ Skolnick. 1962, p. i.

${ }^{26}$ Swanson, 1963 , p. 92

2a Corrado. 1964. p. 110
} 


\section{Wavelength Spectrum A}

$\begin{array}{ll}2289.98 & \text { Ni I } \\ 2300.78 & \text { Ni I } \\ 2310.96 & \text { Ni I } \\ 2312.34 & \text { Ni I } \\ 2313.98 & \text { Ni I } \\ 2317.16 & \text { Ni I } \\ 2320.03 & \text { Ni I } \\ 2321.38 & \text { Ni I } \\ 2325.79 & \text { Ni I } \\ 2329.96 & \text { Ni I } \\ 2337.49 & \text { Ni I } \\ 2337.82 & \text { Ni I } \\ 2345.54 & \text { Ni I } \\ 2346.63 & \text { Ni I } \\ 2347.52 & \text { Ni I }\end{array}$

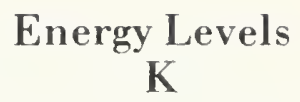

$\underset{10^{8} / \text { sec }}{\text { gA }}$

gf

Log gf
5.0
0.39
$-0.40$
2.2
0.18
$-0.75$
7.6
0.60
$-0.22$
9.5
0.76
$-0.12$
10.
0.82
$-0.09$

$2217-45419$

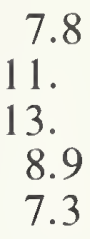

0.63

$-0.20$

$1332-44475$

$0-43090$

0.86

$-0.07$

$2217-45281$

$1332-44315$

1.1

0.04

$2217-45122$

0.72

$-0.14$

$-0.23$

7.3

0.59

$-0.79$

2.0

0.16

$-1.05$

1.1

0.088

$-0.34$

5.5

0.46

$-0.99$

$1332-43933$

1. 2

0.10

0.16

$-0.80$

FigurE 1. Example of computer-typeset tables of physical data.

By this time, also, the Russian All-Union Institute for Scientific Information (VINITI) was evidencing specific interest in computers and high speed composition techniques. Thus, in 1962, Mikhailov reported "development of new highly-productive printing equipment including photo-setting and photo-engraving machines, high-speed mono- and lino-types, composing typewriters", 28 and the like. He also emphasized that: "A comprehensive approach in the advancement of information services presents entirely new objectives to copying and reproduction as compared to those expected of conventional printing practices. There should be developed rapid printing devices transforming into visible characters the pulses received from the information logical machines, ${ }^{29}$

In July 1962, occurred what is probably the first finished book of tables involving the combination of computer processing and automatically controlled photocomposition, NBS Monograph 53, Experimental Transition Probabilities for Spectral Lines of Seventy Elements (Corliss and Bozman, 1962; Bozman, 1963). The introduction to these computer-generated tables of values states in part:

"At the beginning of the preparation of this table it was realized that equipment was available which would permit essentially automatic preparation of the finished book. It was therefore decided to attempt to produce this publication by completely

In Wikhailov, 1962, p. 55

* Ibid. p. 51 . automatic methods. An electronic computer could be used for the computation, then the magnetic tape output from the computer could be used to operate an automatic phototypesetting machine which would produce film ready for making the printing plates." 30

Programs were written for computation of the table values on the IBM 7090 computer and for preparation of an output tape in proper format for the phototypesetter. "The pages were photographically typeset with a Mergenthaler Linofilm machine onto 8 -inch wide film in 50 -foot rolls. About $8 \mathrm{~min}$. were required to set each page. Printing plates were made from the film positives which are actual page size." 31 Figure 1 shows a typical page. It is to be noted, of course, that this first formal book publication employs only a single tabular format throughout.

Also occurring in 1962 were the initiation of the automatic composition program at the University of Newcastle, ${ }^{32}$ preliminary demonstrations of typesetting by Computaprint Ltd. for the British Institute of Printing ${ }^{33}$ and delivery of the prototype Linasec to the Alden Press., ${ }^{34}$ The ROCAPPI (R esearch on $C$ omputer $A$ pplications in the Printing and Publishing Industry) organization initiated a program for automatic control of hot metal line-

\footnotetext{
${ }^{30}$ Corliss and Bozman, 1962, p. xvi.

"lbid.

${ }_{32}$ Duncan, 1964, p. 154.

23 Statement of C. T. Ross, Computer Typesetting Conference "Proceedings", 1965. p. $98-99$.

34 Morton. 1963, p. 96.
} 
casters in October 1962 and went into limited production in January $19633^{35}$

In December 1962, "RCA and the Los Angeles Times jointly announced their computerized newspaper typesetting system. Within the next few weeks, the Palm Beach Post-Times had thrown their 'tape' into the ring." 36

These two newspaper composition applications followed an earlier and less successful venture"the first 'composing room computer' to go on the air was also the first and only to suspend operations." 37 This was the case of the Arizona Journal which, in November 1961, had announced plans to use a GE 225 computer for both typesetting operations and customer billing of classified ads, to begin with the paper's first issue scheduled for February 1962.

Yasaki describes some of the difficulties encountered as follows: "Two weeks before publication date, the rate structure for classified ads had not been firmed-much less programmed. . . . There was cause for concern. A new metropolitan daily ... was beginning with non-union labor to operate newly-introduced offset presses and a new tape perforator, the ATF typesetter. Both were the first installations anywhere. Without experience in the manual processing of advertising copy and billing, the Journal was trying to run a parallel operation with the computer system . . .

"The 225 did not justify lines or hyphenate at ends of lines, these being coded by tape perforators at the Journal office. The information ... . was then transmitted by Bell System's Data Speed to GE's information processing center.

"It was here that some of the trouble lay. Experienced ATF typesetter operators were not available and ... the punched tapes were not proofread before transmission as had been planned. Also, the Typesetter tapes lacked a parity bit. Result: garbage in, garbage out. In the early stages, about 50 percent of the input was unusable. . . ." 38

Although this first combination of perforated tape, computer, cold-type and photo-offset for newspaper composition was in actual use only briefly, the following year, 1963, may be said to mark the major beginnings of automatic composition. As reported in "The Penrose Survey" for 1964: "A number of firms had intalled the Intertype Fotosetter and the Monotype Corporation's Monophoto, but the more elaborate machines still awaited major acceptance. This came in 1963 when a number of installations of the Linofilm and Photon-Lumitype were made . . . the decline of printing from movable type can definitely be associated with 1963 1963 also saw the development and acceptance of setting by computer." 39 In the same Penrose Annual volume in which the survey appeared,

\footnotetext{
${ }^{35}$ Statement of C. Barber, Computer Typesetting Conference "Proceedings", 1965, p. 105.

36 "Computerized Typesetting", 1964, p. 2.

${ }^{37}$ Yasaki, 1963, p. 27.

3k Ibid., pp. 27-28.

: "The Penruse Survey," 1964. pp. 2901-291.
}

Duncan's article 'Look! No hands!' was itself set in accordance with the Newcastle computer program.

In October 1963, the registrants at the annual meeting of the American Documentation Institute received two volumes of short technical articles, claimed to be the first of its kind to be typeset by computer. Figure 2 shows a typical page for a
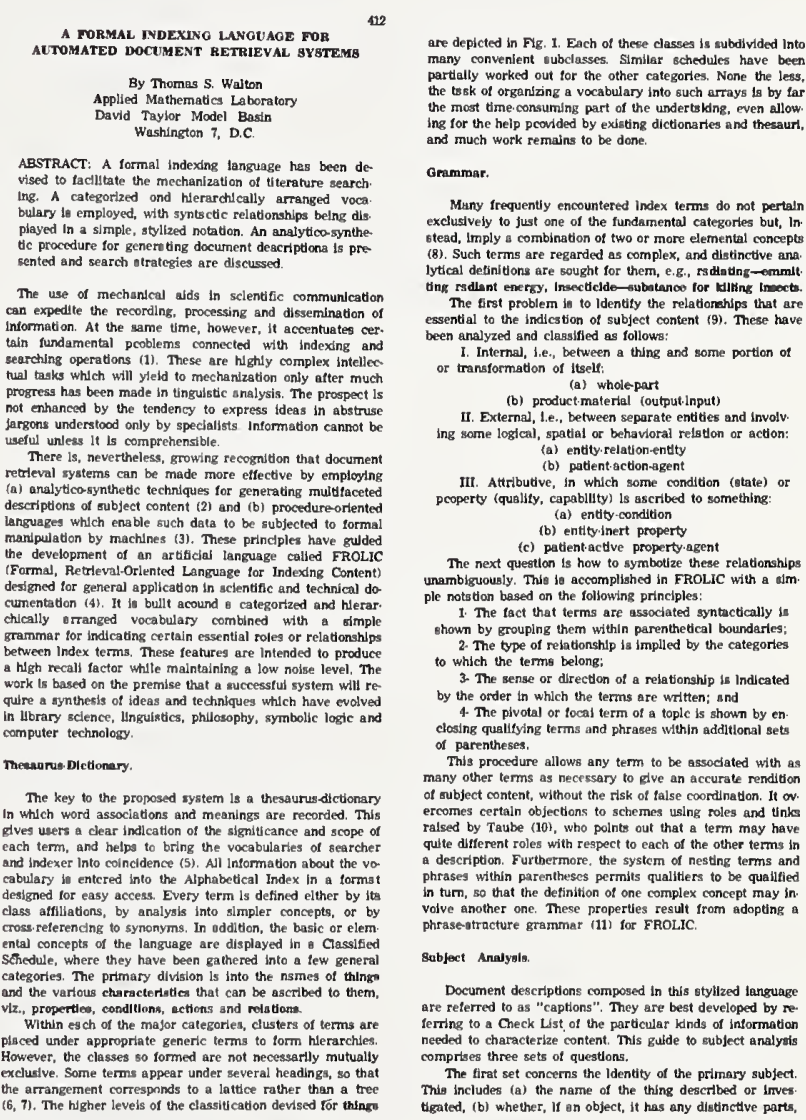

Gammar.

Muny Irequentiy encountered index terms do not pertaln tead, Imply a combination of two or more elementht but, (8). Such terms are regarded as complex, and distinctive ana. lytical definitons are sought for them, e.g, rs ratathg - ominal

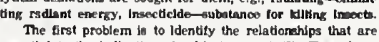
The first problem in to Identify the relationships that are
sisential to the indicstion of subject content 99 . These have

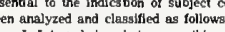

1. Internal, t.e., between a thing and some portion of
I. or transformation of Itseel:

(b) productmaterial (outputinput) II. External, 1.e., between separate entibes and Involy

(a) entity-relation-entty (b) patient-rection-agent III. Attributive, in whlch some condition (itate) Jerty (quality, capabillty) is ascribed to something (b) entity-condilition

(c) patient:active property agent
The next questlon is how to symbotize these relationships nambiguously. This to accomplished in FROLIC with a wim notston based on the following principles:

1. The lact that terms are associated syntactically in hown by grouping them within parenthetical boundares
2. The type of retationship is implied by the categortes so which the terma belong:

3. The sense or direction of a relationship is Indicated the order in which the terms are written; and 4. The plvotal or tocal term of a tople is shown by en ciosing qualitying

This procedure allows any term to be associated with as many other tems as necrssary to glve an accurate rendition of rubject content, without the risk of talse coordination. It ow. ralsed by Taube (10), who polnts out that a term may have quite different roles with respect to each of the other terms in phrases within parentheses permits qualitiers to be qualified
in turn, 30 that the definition of one complex concept may in. volve another one. These properties result from adopting a

Document descriptions composed in this styilized language are referred to as "captions". They are best developed by $x$ ferring to a Checc List of the par ucular kends of intormation comprises three sets of questlons. The firat set concerns the Identity of the primary subject. This includes (a) the name of the thing descrbed or lives:
tigated, (b) whether, if an object, it has any distinctive parts,

Automation and Selentific Communication, Topic 4. Symbolization, Transtormation and Translation of Scientific Information

21

Figure 2. Typical page, "Automation and Scientific Communication, Short Papers, Pt. 1."

special edition of Volume 1. In addition to computer-controlled setting of the text, computer processing was used to produce author, KWIC

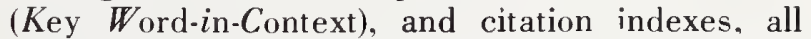
of these operations having been accomplished within three weeks or less from the receipt of author's manuscripts (Luhn, 1963).

Finally, in August 1964, appeared the first issue of the National Library of Medicine's Index Medicus to be computer compiled and automatically typeset at high speed directly from magnetic tape. Figure 3 reproduces the special notice appearing in that issue. 


\section{SPECLAL NOTICE}

This issue of Index Medicus was printed on the Photon 900 Computer Phototypesetter referred to by the Library staff as GRACE (Graphic Arts Composing Equipment).

GRACE transfers data received from the NLM compurer dlrectly onto positive photographic film. It operates at a speed of approximately 300 characters per second, and employs three fonts of type including 6-, 10-, and 14-point sizes in both upper and lower case. The three fonts contain a total of 226 characters, includlng special characters such as dlacritical marks.

The Library wishes to thank the users of the Index Medicus for their patience wlth earlier issues printed by standard computer printer. All subsequent ls sues, including the Cumulated Index Medicus, will be prepared by GRACE.

CHARLES J. AUSTIN

Chief, Data Processing Division

National Library of Medicine

Bethesda, Maryland 20014

August, 1964

Figure 3. Special notice, Index Medicus.

Meanwhile, ignoring the Arizona Journal failure, other news publishing organizations had moved ahead with the installation both of photocomposers and of computer control systems to operate either hot metal casting or photocomposition equipment. ${ }^{40}$ Thus, by 1964. it could be reported that "a recently conducted survey... reveated more than 75 installations involving the computing equipment of some ten different manufacturers.... During the past few years, we have seen the movement of typesetting technology from a relatively stable mechanical craft into a rapidly changing and expanding science engaging principles of electronics, optics, photography, data processing and computers." 11

Additional evidence of the rapidly emerging state of the art is that of labor relation reactions. In July 1964, union printers went on strike in Toronto. ${ }^{42}$ As recently as July 16, 1965. Time reported on the current situation, in part, as follows: "The major obstacles to the on'ush of automation are almost all human. On the one hand, newspaper management has been slow to grasp the importance of the new technology; on the other the labor unions fear that automation will cost them their jobs.... Most papers using computers .. have generally satisfied the union by guaranteeing that no one will lose his job except through attrition. .. Still, some papers already report

\footnotetext{
*Hagan, 1964. p. Ho.

" "Computerized Typeseltin." 1964, p. 3

42 "Signs of the Future?", 1964, p. 88.
}

substantial savings from a reduced work force... At some papers, no printers have lost jobs, not even through attrition... Publishers are eager to take advantage of increased printer productivity." 43

Two distinct technical advances in type composition have thus emerod over the past two decades and especially in the past two years: first, the substitution of photocomposition and/or cathode ray tube character generation coupled with photographic, xerographic, thermographic and other copying processes, and secondly, the use of computer techniques or devices up to and including general purpose high speed digital computers to control either hot metal casting or film-set printing processes. In addition, both types of technological development may be and have been combined in a single application.

A third technological development, that of tie-in to communication networks, has come to practical fruition, from 1963 onward, for stock market exchange quotation and newspaper special edition applications at remote locations, such as the RCA-TTS Dow Jones system, the $W$ all Street Journal system and that of the New York Times. Even more intriguing perhaps, were demonstrations of transmission via communication satellite of news copy. "On the occasion of the American Newspaper Producers Association conference in June 1963, the eleventh and twelfth successful transmissions via Relay I took place between Chicago (the ANPA conference) and several newspapers in Britain, and a similar transmission from Rio de Janeiro to Chicago on 10 June. For these transmissions the RCA 301 computer was employed to justify the setting and to transmit the correct TTS operating codes." 44

Before leaving this area of chronological background we should also note another interesting historical precedent for much later development using more recent technologies which was that of the Thompson patent for an electromagnet typesetting device. As reported in The Inland Printer for April 1899, a number of magnets would be connected in parallel, each corresponding to a specific key of a sending station keyboard by virtue of requiring successively smaller electric currents for energizing them and successively longer periods of time to be energized. Keystrokes would produce in a single electrical circuit currents of different strengths which when the appropriate magnet reactivity is reached cause appropriate typesetter action either directly (keyboard line casting) or indirectly (keyboards producing perforated tape to activate secondary casting equipment). At the same time, the circuit would be broken so that all of the slower-reacting magnets beyond the one to be energized are cut off.

Several advantages were foreseen, but apparently never actually realized. Since a number of machines could be interconnected with a single

\footnotetext{
4a "The Troubled Tide of Autumation." 196.5. p. 42.

44 Duncan. 1964, p. 150.
} 
sending station, it was envisioned that: "Associated Press dispatches could be set up in type simultaneously in various cities by one man operating a keyboard in Washington or any other point. ... If that class of typesetting machines which assemble the type in a continuous line, the justification being subsequently done, were used, the matter of differing measures of newspapers would cut no figure; nor, with that or any other style of.typesetting machine, would the fact that the various newspapers use different fonts in their offices, for with a fat nonpareil a normal minion and a lean brevier, for instance, the same matter could be set in varying fonts in the same measure. . . .

"By having two or more machines in each office equipped with Mr. Thompson's apparatus ... when a breakdown occurs in the receiving machine it could be 'shunted' and another thrown into circuit instantly. ...."45

More than 60 years later, can it be that the spirit of the ingenious John S. Thompson of Chicago, who was himself an "expert linotype operatormachinist," may rest content in latter-day achievements of his dream?

\section{Typographic Quality and the Com- positor Functions}

The history of typography since Gutenberg has been marked by the development of a graphic arts emphasis and by craftsmanship in typeface design based predominantly upon subjective and aesthetic judgments rather than upon objective determinations of comparative readability-legibility.

Nevertheless, as these arts of typographic composition have evolved over the last few centuries, the necessary compromises between quality and printing economy have been centered in the human compositor functions. These reflect, in various balancings, contradictory requirements for aesthetic pleasure, legibility, readability, subject content differentiation and emphasis on the one hand, and for ease, speed, and economy of preparation, processing and publication on the other. In general, "typographic quality is defined by several considerations which are a combination of technical and psychological factors. Most important of these are the choice of typeface, the point size, the use of upper and lower case, the alternation of type faces, and skillful use of the ratio of text to white space surrounding it." 1

More specifically, those compositor functions which enter into the preparation of copy and the setting of type for a printed page of high quality include some or all of the following considerations:

1. Type faces. Variations are used for text, headings, footnotes, captions and the like, and

\footnotetext{
ws "Setting Type by Electricity," 1879, p. 1.

'Sparks et al., 1964, p. 161.
}

detail design reflects such artistic choices as serif embellishments, relative weights (degree of inking or boldness) and spacing of characters and the availability of ligatures (overlapping character pairs). The diversity of type faces that have been developed over the years is underlined by the fact that 325 different designs are provided in the Mergenthaler specimen book. ${ }^{2}$

2. Point sizes - the relative sizes of the characters, from those too small to read without magnification to those used for newspaper banner headlines, for display advertising, and for book jackets. The Mergenthaler specimen book includes in its 325 designs "well over 1500 point sizes, with a range of 4 to 144 points." 3

3. Leading (space between lines). "Variation of interlinear space in type composition is called 'leading,' from the insertion of thin strips of lead between lines of type in the manual typesetting operation." 4 Leading with respect to even column length and placement when two or more columns are to be set on the same page involves vertical justification or column balancing.

4. Line and column widths, in terms of a fixed number of characters and spaces allowed.

5. Intralinear spacing and horizontal justification. This involves left and right margin alignments (whether for pages or columns or both), requiring alterations in the amount of space provided between words and/or that "space is added before and after hyphens inside the line; space is added before and after dashes; space is added between the letters of a word." 5

6. Hyphenation. If all procedures for line justification have failed, it may be required that the 'overset' word (that word exceeding the available line width adjustments) be hyphenated. If so, traditional practice dictates that the hy. phenation occur at a 'proper' point, i.e., one enabling the reader to anticipate that successor part of the word which is carried over to the next line.

7. Page formatting. This typically includes allocations for illustrations, positioning and style of title and other captions, footnotes and bibliographic citations.

8. Treatment of special symbols, equations, chenical structure diagrams, and other interpolations of graphic material not adaptable to line-by-line composition. (This is often regarded as a special case of the page or area composition function.)

9. Line positioning or "quadding," defined as: "positioning of the line with respect to the allocated space, e.g., flush left, flush right, centered, or justified." 6

10. Patterning. ". . . The patterning of typeface ... is a technique of emphasizing words

\footnotetext{
2 Mergenthaler, 1964, p. 3

3 Ibid.

Sparks et al., 1964, p. 168

Sparks et al., 1964.
5 Pay, 1963, p. 330.

5 Pay, 1963, p. 330.
"Sparks et al., 1964, p. 174
} 
by the use of bold or italic faces so that they are easily visible in text. Patterning could well be used in computer composed text as a means for emphasizing search points without resorting to extensive reformatting." ?

More generally, these factors are summarized in the dictum that: "The absolute and relative dimensions of symbols, spaces, and blocks of printing are of prime concern in composition." 8

Further, a number of these factors may be combined in the single criterion of type density. "[It] is measured in the number of characters per square inch of the printed page and is a function of the point size of the type, the characteristics of the font, and the boldness of the typeface. Other factors affecting type density are the intercharacter spaces required for right-hand margin justification and interlinear spacing or leading. Typc density is a measure of the efficiency of typesetting in making use of printing space." 9

The single criterion may also be of value with respect to the introduction and evaluation of computer devices and techniques to be used as aids to the composition operation itself, involving the recognition of necessary difficulties, because of subjectivity, in wbtaining human agreement as to what constitutes "high" typographic quality.

As Duncan emphasizes with respect to typesetting under the control of a general purpose computer: "The writing of a programme presupposes a knowledge of the precise styles that will have to be accommodated by it. . . There is no doubt that programmes would be simple if we had a relatively uniform style. It would, however, be sufficient if we had a consistency in our different styles. At present there is none, and I have yet to find any one feature except perhaps close, even spacing which all agree is a desirable component of style." 10

Garfield remarks: "the proper selection of typrographical style is an important ingredient for consumer acceptance of printed publications. Unfortunately there is no quantitative measure of this extremely subjective consideration - the esthetic and psychological reasons why we accept one format and reject the other." 11

However, although there are no known accurate objective measures of the effects of type density on either effective design or user acceptance, it is recognized, from the human factors point of view, that: "Type density raises psychological considerations. A text which is printed in densely packed type may be difficult to read: on the other hand, text which is dispersed by too much white space between characters and by too much leading between lines can also be difficult to read. Optimum type density is a matter of balancing the

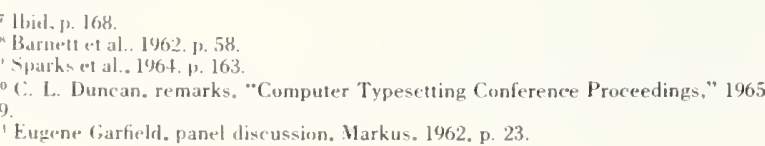

demand of the text to be set with measures of readability." 12

Type density, however, is also related to productivity on the economic side of the typographic quality compromises available. Thus, "obviously, the productivity or density of the type setting will influence the total quantity of the text, considered in units of output pages, and thus the cost of the printing run. Where large quantities of text are involved, close attention to type density may result in savings of many thousands of dollars." 13

The question of quality vs. economy is a crucial one today, especially in terms of the increasingly vast quantity of machine-compiled or machinegenerated output. For example, as Forbes remarked at the 1964 London automatic typesetting conference, ". . . The reason the Government Printing Office got into this business was because we had a great many millions of dollars of composition copy, essentially computer listings, which we are putting on the camera. They are very bulky and they are wasteful. We had to have some means of compressing the information on a tape so we need print only half as many pages." 14

An even more dramatic example than that currently afforded by typical documentation activities, and yet presaging the future, is the following: "One of the services has a stocklist catalog problem involving about $I^{1 / 2}$ billion pages per year of the 19 billion pages of total production. You can see that if we can improve the density by a factor of three, the $1 \frac{1 / 2}{}$ billion pages will be reduced to $1 / 2$ billion pages. Just in terms of paper and ink, using a budget of $\$ 2.00$ per thousand pages, the savings would be $\$ 2$ million per year." 15

Thus, with the advent of high speed automatic data processing equipment capable of compiling numeric tables and listings of alphanumeric material at rates of thousands of characters per second, the dilemma has become more acute-very high speed with poor quality; good quality at speeds uneconomic unless separate devices can be driven in parallel off-line.

Still another factor is the expanding volume of the scientific and technical literature, straining the composition facilities, adding to the cost of technical publications (both books and journals). and increasing the time lag between discovery and disclosure. The problems of the secondary publications, particularly abstracting and indexing journals, have been especially severe. The solution to a part of the overall problem-the emergence of the unpublished report as a major means of scientific and technical communication merely aggravates the difficulties of the abstracting and indexing services with respect both to coverage and timeliness of announcement.

\footnotetext{
1: Sparks et al., 1964. p. 163

${ }^{13} 1$ bid., p. 163

"E. J. Forbes. remarks, "Computer Typesetting Conference Proceedings," 1965 p. 236 .

${ }^{3}$ Corrado. 1964, p. 115
} 
Before considering the machine aids to typographic-quality composition now available we will first review briefly the principal characteristics of hot metal composition and printing, selected cold type systems, photocomposition, and electronic character generation.

\section{Hardware for Typographic Composition}

As noted previously, two different technologies are involved in the present-day advances in high quality type composition. The first is with respect to cold-type and photocomposition methods of reproduction; the second is with respect to specialor general-purpose computer applications in the compositor processes. The two developments are not necessarily congruent.

In this section we shall be concerned primarily with the hardware generally available in the printing and reproduction industries, whether manually, semi-automatically or automatically controlled.

This includes hot-metal casting equipment, certain "cold-type" devices ranging from the office typewriter and sequential card cameras through photocomposition to computer-driven photocompositors and high-speed character generators.

\subsection{Hot Metal Casting Equipment}

The 19th century developments of hot metal casting equipment involved, on the one hand, machines capable of casting a complete line of type at one time and, on the other hand, the Monotype machines that cast a single piece of type at a time and that are operated by a unique 31-channel paper tape. "The hot-lead composing machines developed by Mergenthaler and Lanston in the latter part of the 19th century were the first practical mechanized composing systems."1

\subsubsection{Line Casting Equipment}

In general, the principles of operation of machines that cast a complete line of hot metal type slugs are as follows. First, in line casting equipment, the composing machines are typically equipped with multiple storage units or containers (magazines) which hold supplies of brass casting molds (matrices) for each individual character in the type font or type fonts desired. (Examples of matrices and a cast line are shown in figs. $4 \mathrm{a}$ and $4 \mathrm{~b}$.) As the appropriate key is struck on the keyboard or is actuated by a sensing mechanism which reads the character code from perforated paper tape, the corresponding character matrix is released from its magazine into an assembler. Whenever a full line is completed in this assembler, molten metal is poured into the assembled line of matrices.
A complete, solid line or "slug" of type is then cast and ejected from the mold. After use, the character matrices are automatically returned to their proper places in the storage magazines so that they may be used again.

This operation typically takes less than a minute. High speed line casting machines are typically capable of casting from 375 to 840 lines an hour, when operated under tape control.

Each different face and size of type requires a separate storage container or magazine. However, present-day machines are built to hold several magazines at any one time, so that a variety of type sizes and faces can be available for a given application.
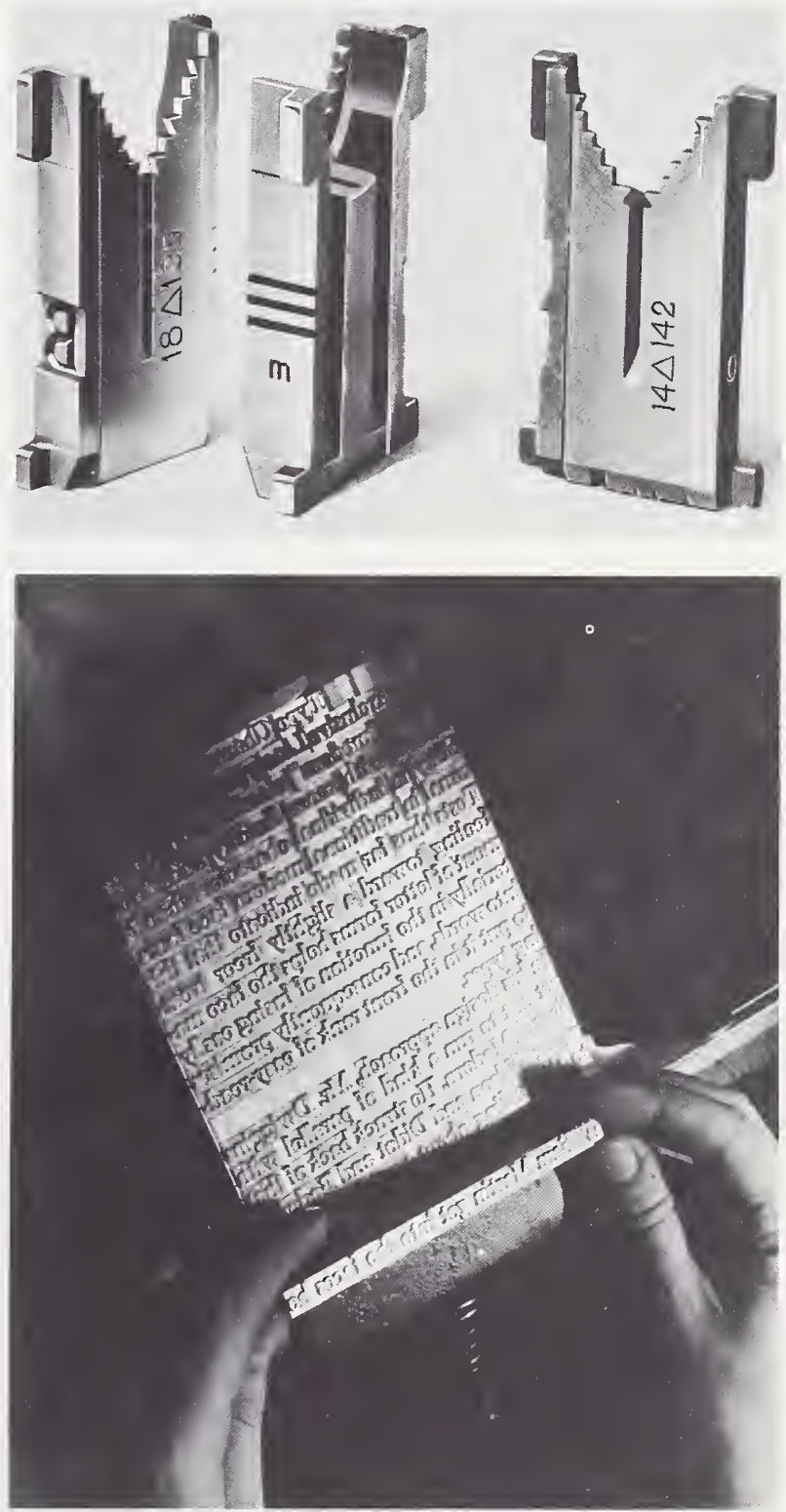
There are basically two kinds of line casting machines. They are, respectively, mixer (multifont) or nonmixer machines. The number of magazines which can be held on a mixer machine is typically eight. Thus, there are four main magazines with 90 channels each and four side magazines with 34 characters each. The total number of channels, therefore, is 496. If dual letter (upper and lower rail) matrices are used, the maximum number of characters that can be mixed is typically 988. (While this is a considerable number, it is still small in comparison to the number available with photocomposition techniques because of the availability in the latter systems of variable magnification which can be used to achieve a variety of point sizes for any of 1400-1500 characters.)

Since hot lead line casting machines require a separate matrix for each character in each size and in each font, it is necessary that there be a reasonably large quantity of each individual matrix stored so that the supply will not run out before the matrices are returned and redistributed after use. The supplies of the various matrices must eventually be replenished due to normal wear and breakage. When standing lead must be stored for repetitive use in frequently updated and reprinted publications, the volume and weight in lead and in other supplies becomes a considerable problem.

Manually controlled hot-metal line-casting has been generally available for over 70 years and tape operation of linecasting machines has been available since 1927. As a result, a wealth of technical and descriptive material is available that adequately describes this composition technique.

There are currently two major manufacturers of line casting equipment: the Mergenthaler Lino-

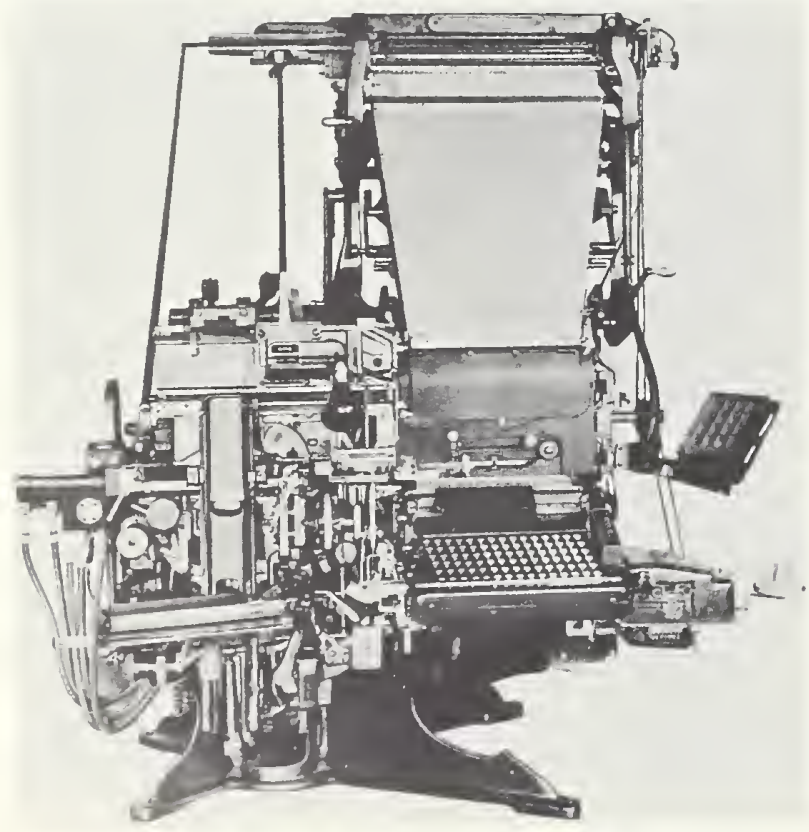

Figure 5. Linotype Comet. type Company and the Harris-Intertype Company. In the late 1920's and the early 1930's, tape-operation of line-casting equipment was successfully introduced for line-casting equipment. The present Linotype line has several tape-operated line casters including the Comet (Fig. 5), the Meteor, the 31 Race Horse, the Rangemaster 33 and 35, the Model 29 Blue Streak, and the Elektron. Intertype offers five machines (see Fig. 6) for automatic operation including the Monarch, the Model C, the Model V, the Model F, and the Model G.

Automatically controlled line-casting involves the use of a tape-interpreting unit attached directly to the composing machine. As the perforated tape runs through this unit, a sensing mechanism reads the code perforations which have been punched for each character and automatically translates them into key-release movements performing the same functions that would be carried out under manual keyboard operation. The operating interpretation unit is thus basically a paper tape reader that actuates the keys to release matrices. It also functions to control the upper or the lower rail in the assembling elevator (e.g., to select italic or boldface rather than Roman versions of a character), and to operate an automatic "quadder" which computes the space values necessary to center or justify a line.

The better known tape-operating units are:

Fairchild Graphic Teletypesetter

Star Parts Autosetter

RCA Electrosetter

Mergenthaler Linomatic Operating Unit

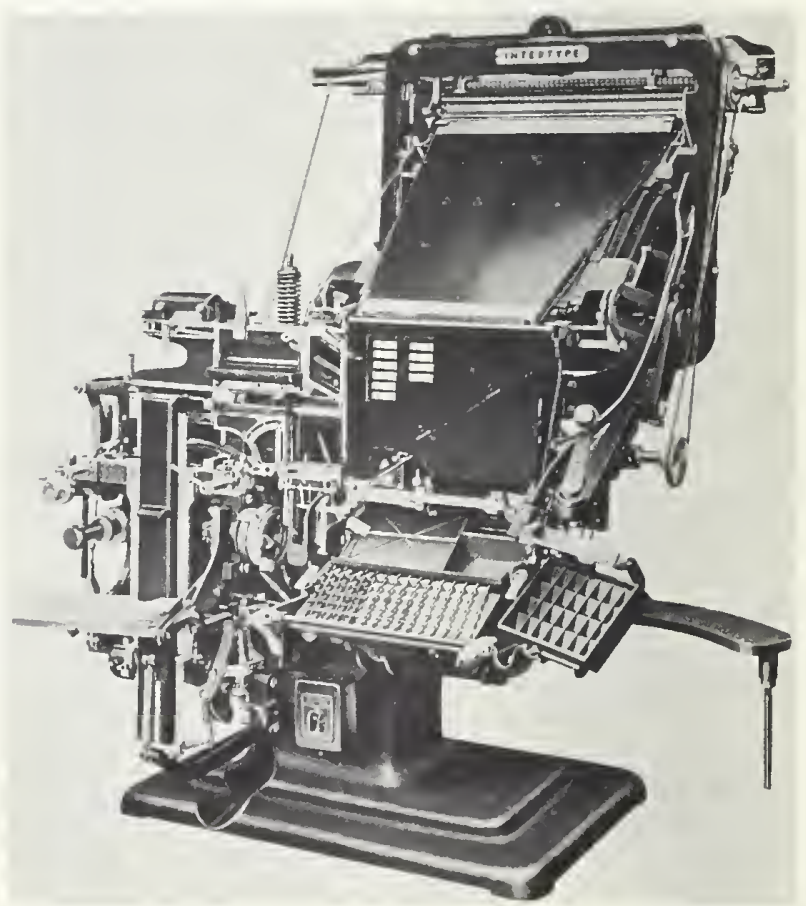

Ficure 6. Intertype Linecaster. 


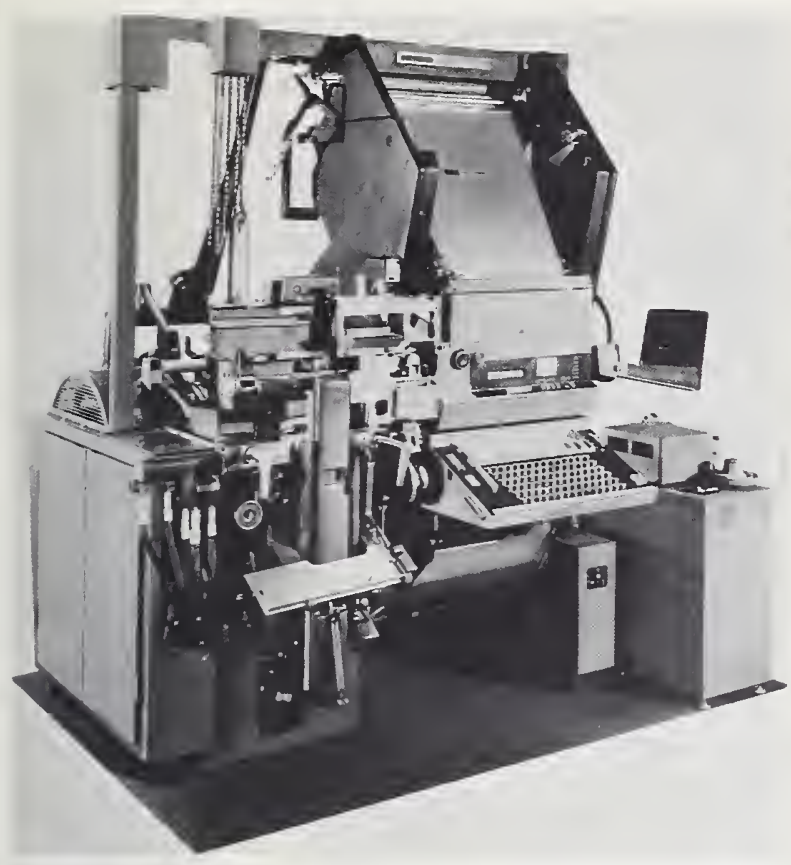

Figure 7. Linotype Elektron.

The Linotype Elektron (shown in Fig. 7) is the fastest slug casting machine currently made and it is available in mixer models. It operates at a maximum speed of 15 lines of single column newspaper copy per minute. Prior to the introduction of the Elektron in April 1962, the Intertype Monarch, which can deliver up to 14 lines per minute, was the fastest and most advanced line casting machine. The Monarch can carry two, three, or four magazines at one time. Converted into characters per second, the tape operating speeds of hot lead line casting machines are still a very moderate 7 to 10 characters per second in contrast to the new computer output graphic arts composers which are capable of operating at speeds above 500 characters per second.

Moreover, as Yasaki comments: "Reputedly the fastest typesetter is the Linotype Elektron, which casts 15 lines per minute from computer tapes. This contrasts with seven to eight lines from manually-operated machines. But if an hour of computer time will produce sufficient tape to keep a battery of linecasters running all day, even the Elektron becomes a weak link." 2

\subsubsection{Tape-Controlled Hot Metal Typesetters}

Returning now to the other 19th century hot metal casting developments, we note first that the Monotype $^{3}$ is probably the oldest tape-operated composing machine in existence, followed in time by

${ }^{2}$ Yasaki, 1963, p. 31.

3 "Monotype Composition Caster," 1961

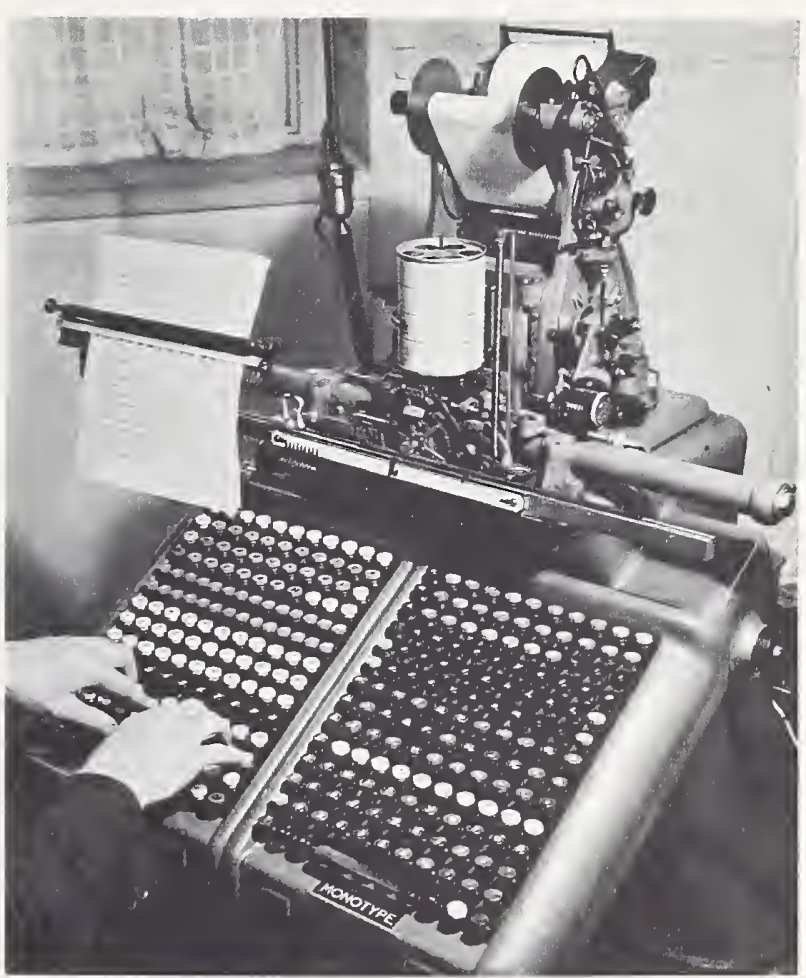

Figure 8. Monotype keyboard.

tape-controlled accessories for the Linotype ${ }^{4}$ and Intertype ${ }^{5}$ line casters.

The most significant feature of the Monotype by comparison with its linecasting competitors is that it composes by casting single pieces of type rather than entire, solid lines. Characters and spaces must be assembled to provide justified lines of equal width for the column or page. The single matrix casting principle makes it easier to make simple corrections in type as it is cast (such as reversing the order of accidentally transposed characters) than is the case with the linecasting processes. It is also relatively easy to insert special characters as required in the hand-setting of difficult composition work involving mathematical equations or other special symbols.

The Monotype keyboard is a unit that is separate from the lead caster proper. As shown in Figure 8, it resembles a very large typewriter carrying a vertical graduated drum and a horizontal scale which progressively indicates the total amount of space left in the line as the characters and spaces being set are accumulated. When the full line has been completed, the keyboard operator presses special control buttons in accordance with the indication of a rising pointer on the drum, which has rotated in the meantime. The perforated paper tape ribbon is then punched with the line justifica-

"Mergenthaler Linotype Co., "The Linotype Line", n.d

sIntertype Co., "Intertype Linecasting Machines", n.d 


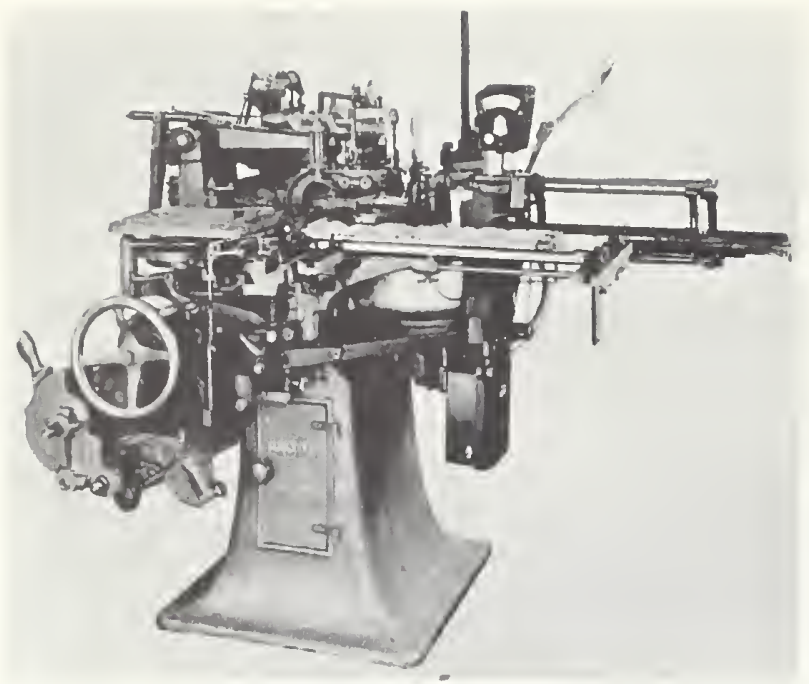

Figure 9. Monotype caster.

tion signals required by the lead caster (shown in Fig. 9).

The Monotype caster must receive these line justification signals before it can commence casting the line. For this reason, the caster reads the punched paper tape ribbon in a reverse direction. That is, the last operator action made on the keyboard for a given line results in the first signal used to control the operation of the caster as it composes that line.

The character matrices for Monotype (as in the case of slug-casting techniques these are small bronze molds for each character) are mounted in a matrix case small enough to be held in the palm of the hand (Fig. 10). For type sizes up to 14 point, there is room in this case for 255 individual matrices which are arranged in 17 rows of 15 matrices each. A typical piece of final printed text may thus involve a choice of up to 255 different characters consisting, for example, of upper and lower case alphabetics, numerics, punctuation marks and selected special symbols, which are available in Roman, bold face, and italic versions. With the Monotype composition caster, up to seven different alphabets are available in one typesetting operation.

The Monotype perforated paper tape which operates the caster, however, "is incompatible with most computing equipment" 6 since it uses a 31-bit code and a tape width of $45 / 16$ inches. Difficulties in terms of accepting Monotype tape sources of potentially computer-usable text are obvious, even aside from the more difficult problems of handwritten indications to compositors to change fonts and to insert corrections where there is a high incidence of hand-corrected typographical errors. It is also obviously difficult to automate, or to provide computer-prepared input for, a Monotype printing plant.

"Sparks et al., 1964, p. 171.

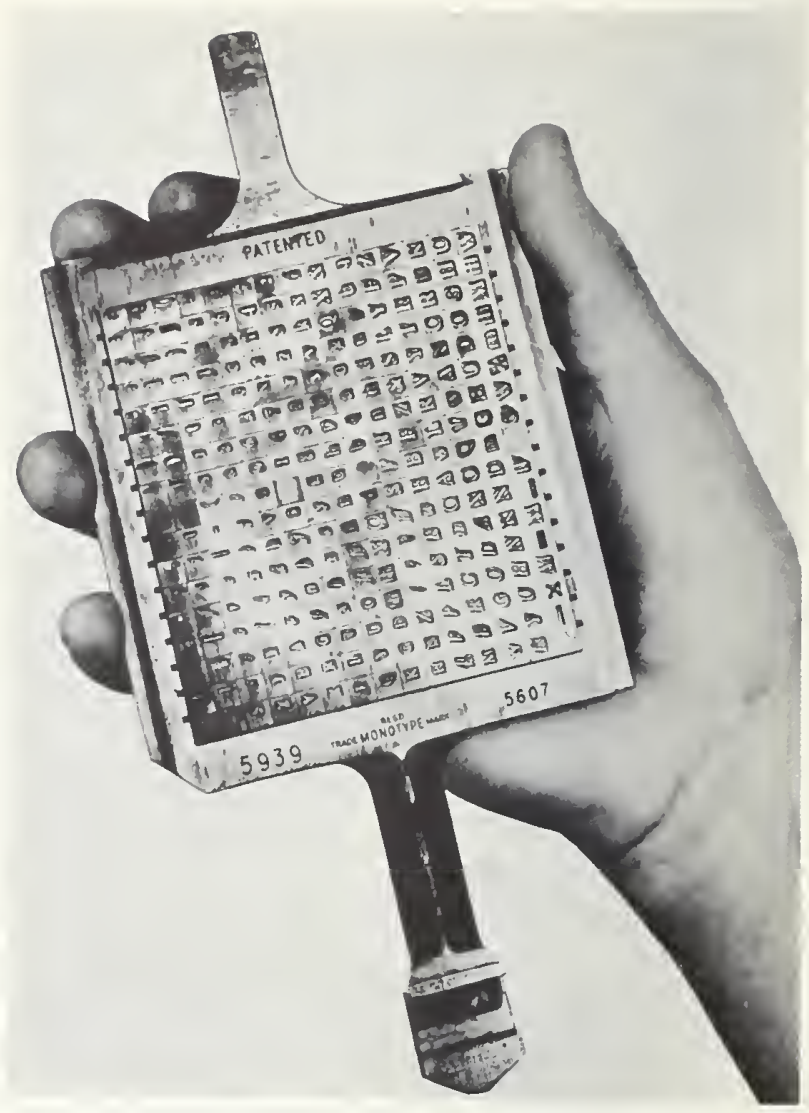

Figure 10. Monotype matrix case.

Duncan discusses three possible methods of automating such a plant as follows:

"The first is to replace the keyboard by a typewriter input, calculator, and thirty-one-hole punch. This is available as a commercial system, "Monotron', developed by Walter Bally from Guttinger in Switzerland. It aims at typewriter input speed with simultaneous hard copy and smoothed continuous caster output with accurately spaced and justified lines. Word splitting is decided at the input stage by the typist compositor but a number of timesaving automatic display instructions can be keyed in from the console. . . The second method is to convert the eight-hole tape produced by computers into thirty-one-hole 'Monotype' control tape - this has only been done experimentally so far at Newcastle. ... The third method, attempted so far only by Bafour, is to control the caster or setter directly from five- or eight-hole tape without the intervention of the unnecessary thirty-one-hole roll." 7

It is interesting to note that the Photon Corporation has introduced a tape-operated Photon photo unit which will operate directly from Monotype tapes. 'This development resulted from the recognition on the part of the Photon Corporation that a large number of Monotype keyboards are in use

${ }^{7}$ Duncan, 1964, p, 141 
today and represent substantial capital investments. The new Photon photo units will make it possible for composition houses to replace their Monotype casters without having to dispose of the Monotype keyboard units. Likewise, the Monotype keyboard operators will not need special training in photocomposition techniques.

In the case of line-casting equipment, by contrast with Monotype, the perforated tape control systems are compatible to a greater or lesser extent with 6-, 7 - and 8-channel tapes used in other machine processing or in communication systems. In particular, many of them use, or can be made compatible with, the 6-bit Teletypesetter (TTS) system and code format. In order to utilize standard perforators for automatic tape operation of these slug casting machines, Mr. Walter Morey, the inventor of the TTS system, with the cooperation of the Linotype and Intertype Companies, has developed a unit counting system similar to that used by Monotype. Each character is assigned a unit value regardless of its size or face. This is a relative width value, and the system usually involves 18 units of width to the em quad. An em is usually defined as the area occupied by the capital letter $\mathrm{M}$ in a given font of type. Each unit therefore is $1 / 18$ of an em. The Linotype and Intertype Companies have developed whole families of unit cut fonts which were designed on the 18 unit relative width system. These unit cut fonts can therefore be composed at a standard TTS keyboard because the upper magazine and lower magazine fonts are compatible.

\subsection{Cold Type Techniques}

From the combining of office typewriter paper sheet output with relatively inexpensive, officeoperatable reproduction devices (Thermofax, Ultrafax, Xerox, Multigraph, Duplimat, and a dozen or more others) there has been developed a wide variety of non-hot-metal techniques available for multiple copy preparation of material for distribution or publication. ${ }^{8}$

The first practical alternative to hot metal casting and printing of telephone books. directories, catalogs, bibliographies. announcement journals, indexes, and similar materials requiring re-assembly and up-dating before re-issue in large editions, was the coupling of photographic techniques with either manual or semiautomatic "shingling" techniques. For example. in the preparation of the Bibliography of Agriculture. "each of the author slips is pasted onto the Master Sheet. one on top of the other so as to expose only the top line of print containing the author's name and citation number. This process is called shingling". 9

\footnotetext{
"See fur example. "Office Cupying - A Technology in Flux", Chemical and Engineer ing lews for July 13 and July 20.1964.

"L.S. Department of Agriculture. "ABLE". 1965. p. 5.
}

\subsubsection{Sequential Card Camera Systems}

The need for updating and recomposition of lists, catalogs, indexes, directories, and the like led to the development of the sequential card camera technique. The manual shingling technique employed in producing cumulative indexes served to eliminate the need for retyping the entire index at the end of each period of cumulation; however, shingling is notoriously time consuming.

The sequential card camera, in effect, automatically shingles the cards and photographs the composite listing on a galley of film. The use of tabulating cards and punched card equipment provides the added flexibility of sorting and merging by machine.

An excellent writeup of the sequential card camera method formerly used for producing Index Medicus was published in the January 1961 issue of the Bulletin of the Medical Library Association. That article describes the equipment and processing, the method of punched card preparation, and the operational history, and it provides a detailed cost analysis and a general appraisal of the system.

The sequential card camera systems typically involve three basic steps prior to printing. These are:

(1) Composing the copy on camera-ready cards that can be manually interfiled with the cards stored for previous copy.

(2) Page makeup (specification of column lengths and pagination, and insertion of header and space cards).

(3) Automatic assembly onto film (listing the cards on film).

Individual lines of copy are composed on cards by ordinary office typewriter, by Varityper equipment, or even by a Linotype and proof press for bold headings. and the like. In some systems, several multiple lines of information can be composed on and simultaneously copied from the same card. Accurate placement or registration of the copy on the card is a critical factor. Consequently, special card-holding platens have been designed for various typewriters. The Addressograph-IIultigraph Corporation's subsidiary. Varityper Corporation, manufactures a special model known as the Line Composer for use with its Fotolist system. The Fotolist has a "pin gripper" method of registration. That is, the cards have pre-punched round and square holes which are used for accurate alignment both by the Line Composer and by the Fotolist camera. Standard punched cards are frequently used because of the increased flexibility which can be achieved by machine sorting and collating when special sequencing codes are punched into the card in a non-image area. Keypunching makes it possible to produce cumulative lists by automatically merging additions to the file or to produce special geographical or other classified lists by sorting out the appropriate cards on punched card equipment. 
There are, of course, some corresponding disadvantages. Typically, "the actual text printed on the EAM card is not punched on the card, and the use of text on the card eliminates some of the columns for punching of data." 10

The page makeup step involves inserting heading cards and page number cards and, if required, column spacers to prevent splitting an entry in the front of each deck or column of cards, and then counting the appropriate number of lines of copy which are to appear on the column or page. This is usually done on a Pitney Bowes Tickometer. Once the cards have been counted, they are refiled with separator tabs indicating the proper page number, ready for automatic listing by the sequential card camera.

At publication time, the cards are removed from their files and placed in the hopper of the sequential card cameras. The camera automatically photographs the appropriate area of the cards (one, two, or more lines depending on the special device) at rates ranging up to 14,000 cards per hour. The product of the camera operation is an exposed photographic negative which contains the information appearing graphically on the cards in the desired sequence. The negative can then be processed in a darkroom or, more typically, in an automatic film processor. All of the sequential card cameras available can produce a paper positive for proofing as well as a film negative.

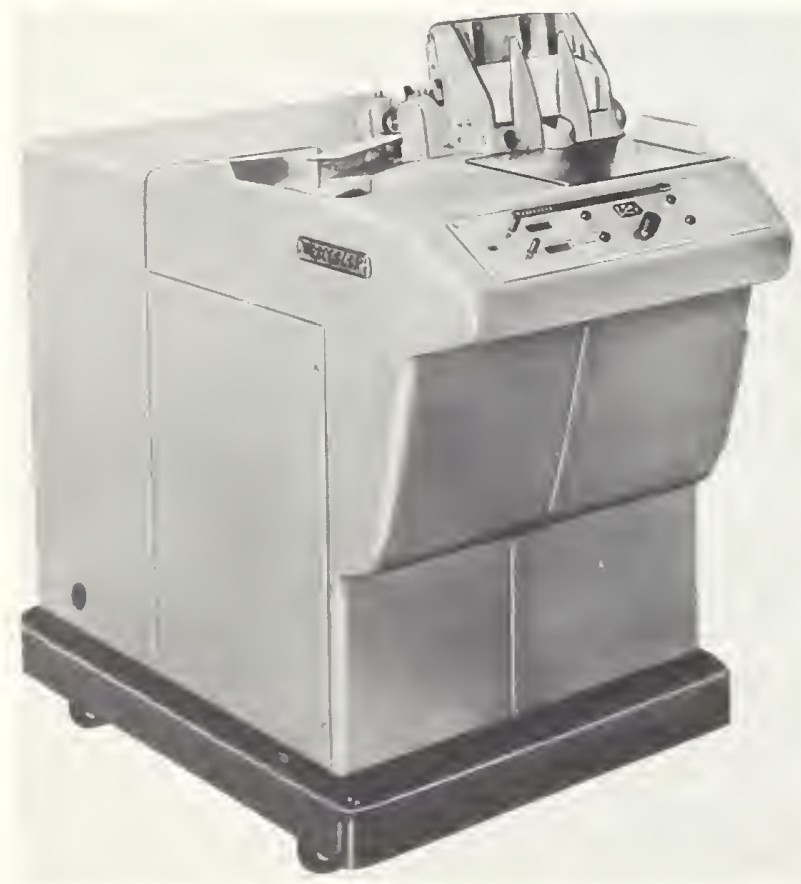

Figure 11. Recordak Listomatic.
The sequential card camera can photographically reduce or enlarge the size of the copy on the card. In some applications, special anamorphic lenses are employed to reduce the copy horizontally rather than proportionally in all directions, thereby producing the effect of squeezing the type together. This is particularly useful in reducing the column width proportionately more than the character height when photographing punched cards that are self-interpreted (printed), with one character per punched column, so that characters are spaced relatively far apart.

There are at least three commercially available series of cameras. These are the Recordak Listomatic as shown in Figure 11, the Varityper Fotolist as shown in Figure 12, and the Friden Compos-oline as shown in Figure 13. Each of these will be described briefly and some of their similarities and differences pointed out.

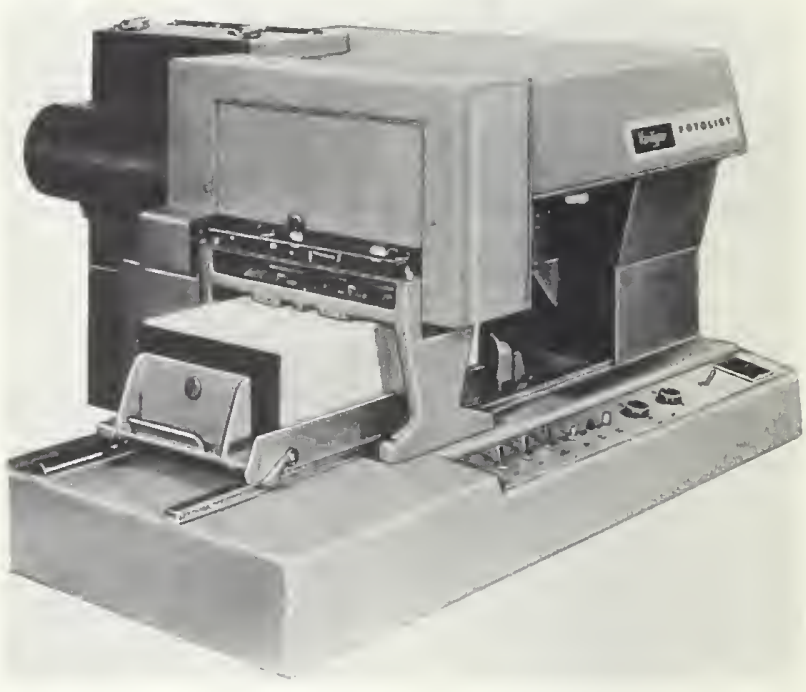

FIGURE 12. Fotolist.

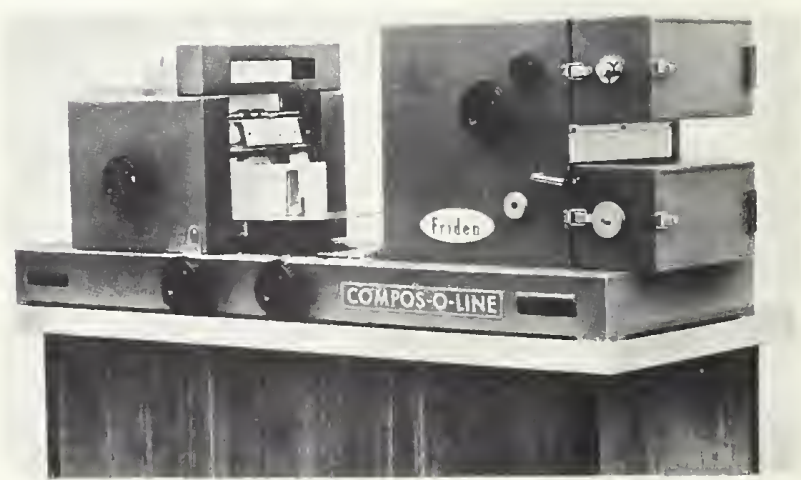

Fil.URe 13. Compos-o-line. 
The most distinctive feature of the Listomatic camera is its ability to intermix one to three lines of copy per card at a rated speed of up to 13,800 cards per hour. This is in response to "an appropriately positioned keypunch". 11 The Fotolist camera has a system for accurately positioning the text on the card. This involves a pair of round and square holes in each card which are engaged by pin gripping mechanisms in both the keyboard and the camera. The Compos-o-line's chief virtues are its ability to handle many sizes of cards and to photograph a larger area of the card than its competitor devices. "The area of the card to be photographed may be selected for any given run." 12

It should be noted, however, that "with the Fotolist and Compos-o-line cameras, the aperture can be set for a desired number of lines beforehand but cannot be changed automatically during a run." 13

Several automatic film processors are available including an automatic developer for the Fotolist, a Recordak Listomatic processor, and a high quality continuous processor by Oscar Fischer, Inc.

Applications of Fotolist, Listomatic and Composo-list systems have included, in addition to Index Medicus from 1961 to 1964 (when GRACE highspeed photocomposition techniques were introduced), the Index Handbook of Cardiovascular Agents, produced by IBM typewriter with modified card-holding platen and by Listomatic camera, ${ }^{14}$ Nuclear Science Abstracts, and International Aerospace Abstracts.

Duncan reports: "In 1961 the British National Bibliography introduced the Fotolist camera using eighty-column cards with entries typed on Vari. typers (one line to each card) to produce the cumulative annual volumes of the BNB from intercalated copy from the separate issues. It was so satisfactory as compared to hot metal, that the unit now handles the British Technology Index, the British Humanities Index, the British Education Index, and the British National Film Catalogue, as well as an increasing number of directories and membership lists of all kinds." 15

Coates cites a special advantage from the standpoint of timeliness of secondary publications. "The non-conventional method by which the British Technology Index is produced probably varies little in cost from the conventional processes with single keyboarding ... However, the peculiar virtue of this process, to us, is that is saves time. This is all-important in our case because the utility of our publication is very much bound up with the shortness of time-lag between the date upon which an article appears in the technical press and the date of the B.T.I. issue which records it." 16

\footnotetext{
"Markus, 1962, p. 17.

12 Rogers, 1964, p. 191

13 Markus, 1962, p. 17.

${ }^{13}$ Markus, 1962,1
is Ibid., p. 18.

is Duncan, 1964, p. 148

${ }^{16}$ Coates, 1965 , p. 45.
}

With respect to other British and West European developments Duncan notes several simple 'cardshuffling' systems such as the Remington-Flexoprint and the Kalamazoo-Photostrip. He describes operation and application of the Dutch NHM (NoHot-Metal) system as follows: "The NHM system proper was intended for web offset newspaper production and originally comprised a special version of the IBM Executive typewriter with a special register platen to allow the typed camera copy produced to be spliced together accurately for make up, a special justifying camera using anamorphic optical stretching and headliner device and an editing table with a cathode ray tube display which seemed to foreshadow the devices reported from America for newspaper mark-up.

"[The] NHM Cartoprint . . . has been used in Denmark for telephone directories (a similar system has also been used in Holland) and part of it in this country to print the Woking Telephone Directory (December 1961) and the gazetteer to Philips G. B. Road Atlas of Great Britain (six miles to the inch1962). The cards in this case are typed in a continuous web which is subsequently cut and punched with registration tags, assembled on metal storage boards, and photographed column by column in pre-set page array by a special camera at five columns a minute." 17

Many commercial composition houses offer sequential card camera services on a service bureau basis. Running charges usually average about 1 to $1 \frac{1}{2}$ cents per card, plus the cost of film. An advantage of service bureau operation is that it eliminates the necessity for hiring specialized personnel to operate and maintain the camera and processor. The customer supplies the camera-ready cards and receives back either columns of film or completely stripped up galleys.

\subsubsection{Ribbon Impression Composing Machines (Without Tape Perforators)}

Typewriters and other ribbon impression composing devices may be divided into two major groups; those that utilize perforated tapes or cards, and those that do not. The standard office electric typewriters are monospacing devices and are therefore not included in this report. Computer line printers are likewise monospacing and are not included, with the exception of certain chain printer applications which involve simulation of boldface and more versatile character sets than the uppercase-only limitation found on most high speed printers.

The IBM Executive is distinguished from other electric typewriters in that it is a proportional spacing machine. There are a maximum of only five different spacing values, however, whereas in quality printing there are usually 18 units of width, and sometimes 36 or more. (On the IBM Executive, for example, the capital letter "M" would

\footnotetext{
${ }^{17}$ Duncan, 1964, p. 148.
} 
occupy the largest of the five relative width values whereas the small "i" would occupy the smallest width value.) The significance of this feature for typescript, however, is two-fold: (1) it improves the appearance of the printed page; and (2) it will pack $10-15 \%$ more characters onto the page.

The IBM Executive is not equipped for perforating paper tapes. It is, however, suitable for typing cards for sequential card camera systems, or for typing text to be photographed for reproduction, offering the obvious advantage of proportional spacing over monospacing typewriters or tabulator interpretation of keypunched characters.

Another machine which offers proportional spacing, but not tape perforation, is the $V$ arityper. The Varityper is perhaps the most versatile of the cold type machines. Its primary disadvantage is that it cannot be operated by paper tape. It is quite suitable, however, as a partner to the sequential card camera. The Varityper has easily changeable type fonts, two of which can be mounted on the machine at any one time. This feature makes it possible to intermix light and bold face with Roman and italies plus many special symbols, superscripts, subscripts, and the like.

Compared to an electric typewriter the Varityper keyboard has a rather heavy touch, and the composing speed is somewhat slower because of the need to make font changes and the requirements for the manual insertion of each card individually. Horizontal spacing of characters is variable in four increments up to an escapement of $1 / 54$ inch. A variety of contensed type faces are available which can produce more characters of copy per page than any electric typewriter available. Justification is computed automatically (but a retyping is required to justify) and spacing is variable from 0 to 18 points. The Model 100 Varityper has a specially designed card holder which accepts a tabulating card prepunched with round and square alignment holes in columns 1 and 80 of the card to provide good registration.

\subsubsection{Perforated Paper Tape Systems}

Turning now to the perforated tape equipment for control of casting machines, it is to be noted that keyboard operated devices producing the perforated paper tape are sometimes combined with a typewriter to provide proof copy. In other cases, a contrary opinion is advanced that the proof copy is considered to be a distraction to the keyboard operator and no printout mechanism is included. Such nonprinting perforators, however, usually provide right column justification information to aid the operator in the preparation of the copy.

Punched card perforators are not included in this report, because until 1965 conventional punched cards had such a restricted repertoire that they could not represent both upper and lower case letters as unique punching patterns in a single column.
The Alphatype photocomposer employs a special magnetic tape. It is omitted in this section, but described in the section on photocomposing machines.

Perforated paper tape output systems are commonly used:

(a) to drive a hot metal or phototypesetting machine.

(b) as input to a special purpose hyphenationjustification computer.

(c) as input to a general purpose digital computer designed or programmed for some degree of typography computation.

\subsubsection{Tape Perfolators Without Printers}

Composition tape perforators which do not have cold type printers include the following:

Fairchild Graphic Standard Teletypesetter Perforator

Fairchild Graphic Multiface Perforator

Mergenthaler Linomatic Perforator

ATF Typesetter Keyboard

Star Parts Perforator

Lanston Monotype Narrow Keyboard

Some of these keyboards are shown in Figures 14-18.

The Monotype wide tape keyboard produces a perforated paper tape $4 \frac{5}{16}$ inches wide containing 31 information hole positions. This tape contains information on 255 selectable characters, and their proportional spacing to provide right column justification. These tapes can drive a Monotype hot metal type casting machine, or a Photon photocomposing machine adapted to read these tapes. In England, the Ferranti Pegasus computer has been equipped to handle these 31 channel Monotype tapes, and in the United States at least one attempt has been made to use these tapes as computer in-

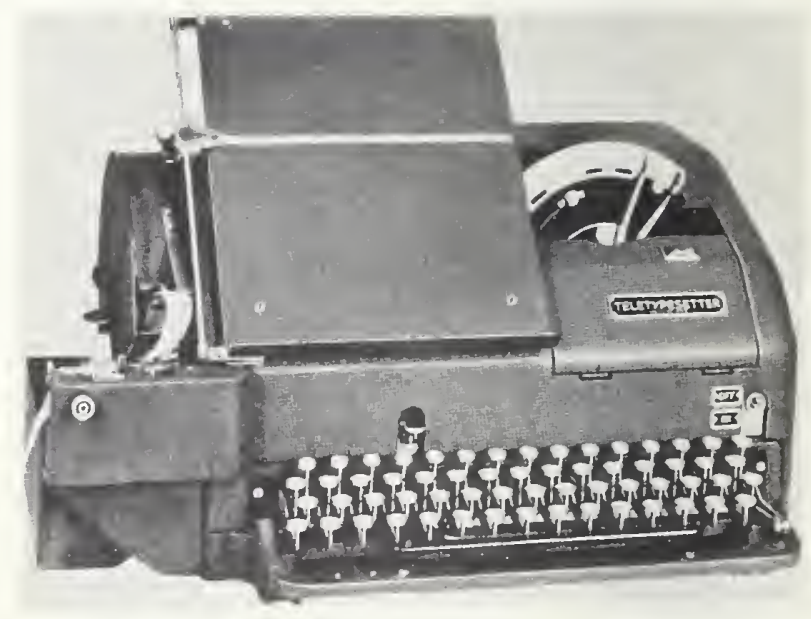

Figure 14. Fairchild (iraphic Standard Teletypesetter perforator. 


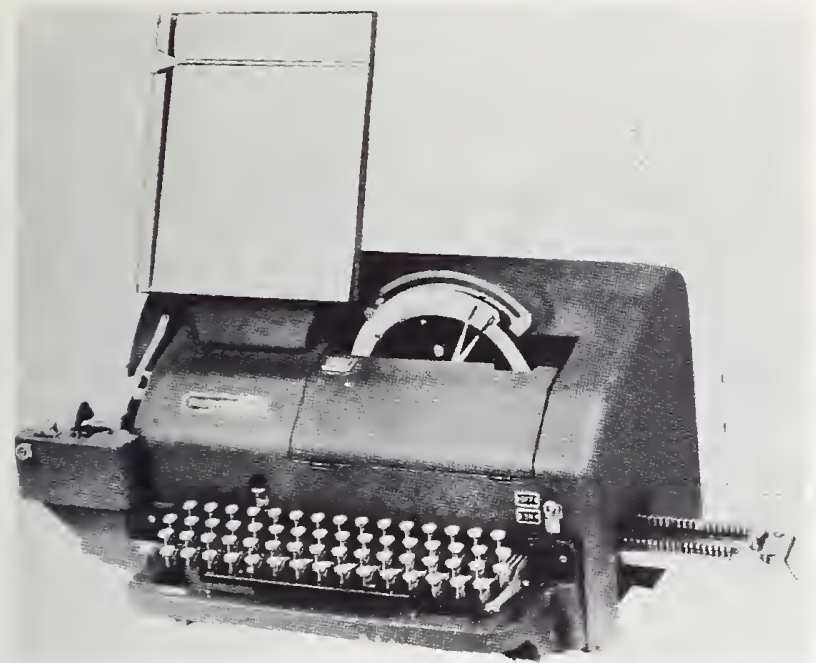

Figure 15. Fairchild Graphic Multiface perforator.

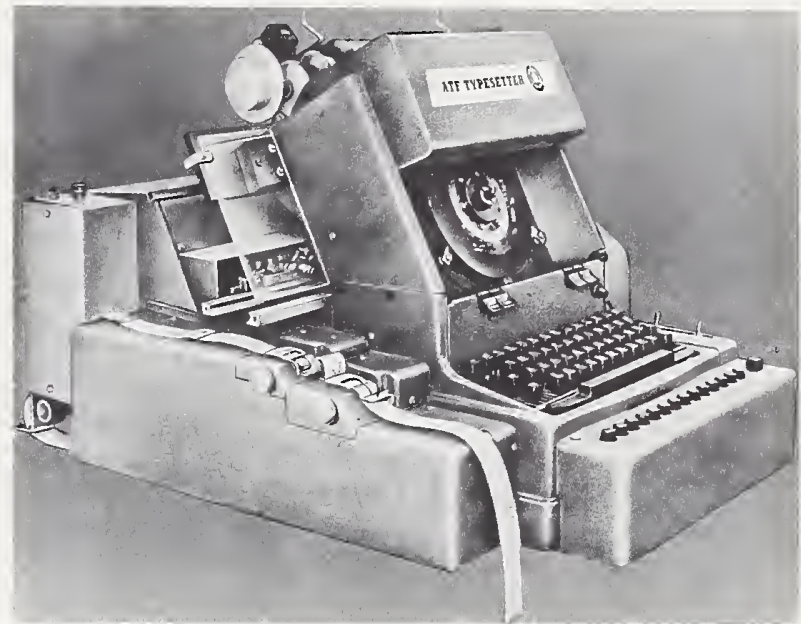

Figure 17. ATF Typesetter.

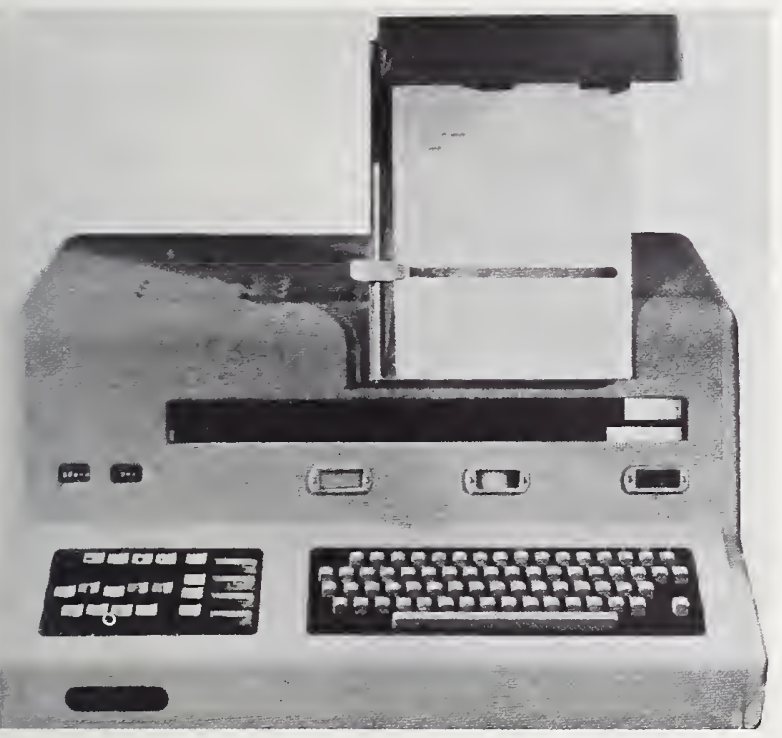

FiguRE 16. Mergenthaler Linomatic perforator.

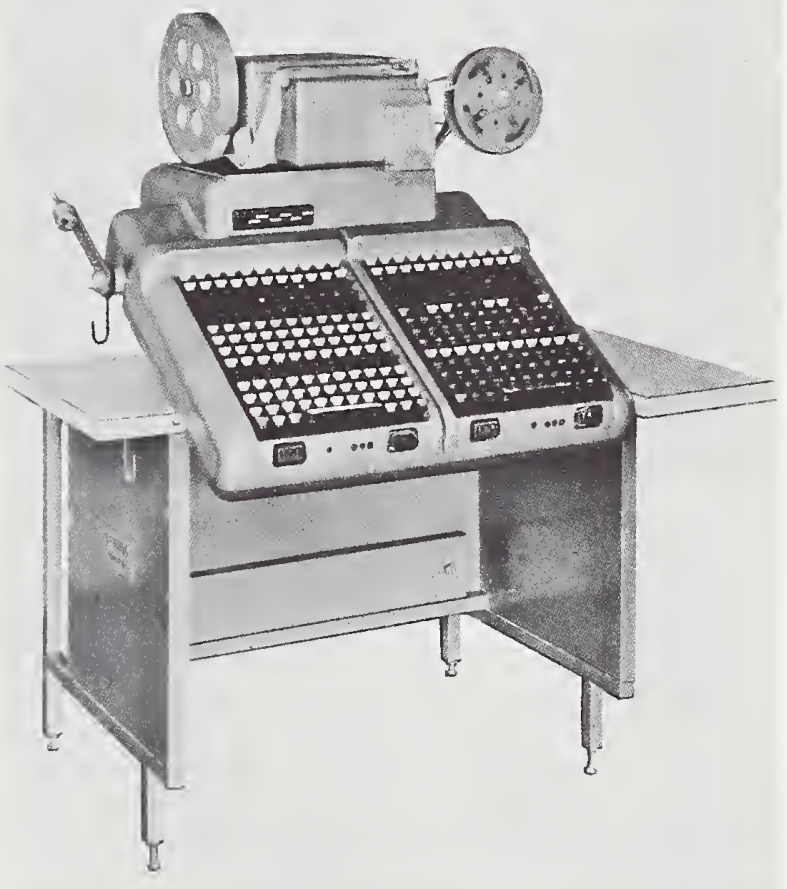

FIGURE 18. Monotype narrow tape keyboard.

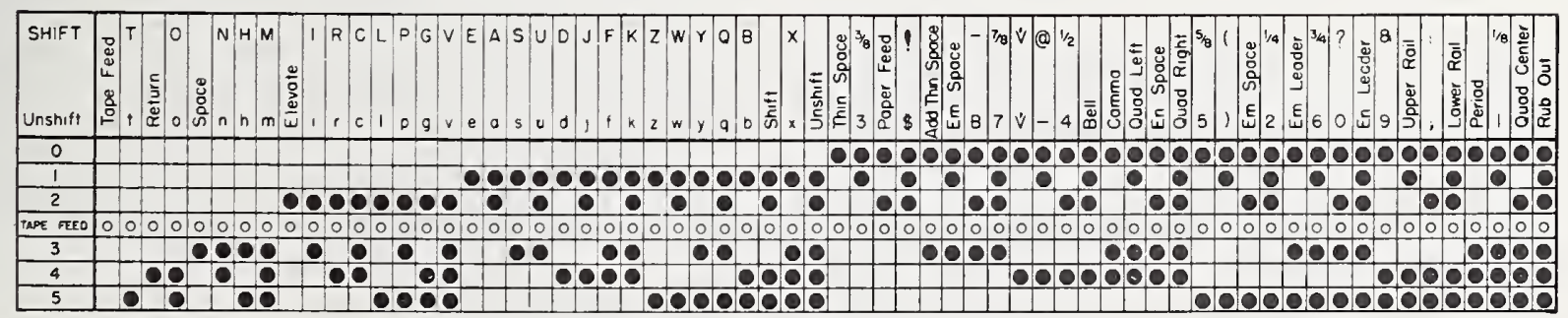

FIGURE 19. The Teletypesetter (TTS) code. 
put. The Monotype narrow tape keyboard shown, however, is compatible with the standard teletypesetter system.

\subsubsection{Teletypesetter (TTS) Systems}

All of the tape perforators above except the Monotype wide tape unit can generate a 6-hole Teletypesetter tape $7 / 8$ inch wide, with 64 unique code patterns. The Teletypesetter (TTS) system was introduced in 1932 by the Teletype Corporation, which later divested its interests to the Fairchild Graphic Equipment Division of the Fairchild Camera \& Instrument Corporation.

Teletypesetter tapes are widely used to transmit newspaper copy via communication networks, including news stories and such material as stock market tables. Tapes can be reproduced at distant newspaper locations, and these tapes can be fed directly into hot metal or phototypesetting machines that have been adapted for this purpose to produce final copy, fully justified and formatted. Suitable type styles and point sizes can be selected to match the desired column widths, which need not be the same for the different newspapers receiving the same initial copy.

Unlike most other perforated paper tapes. Teletypesetter tapes employ an advanced-feed sprocket hole so that the tapes cannot be read if they are introduced into the reader with the trailing end first. Ironically, however, the tapes are symmetrical, with three code hole positions on either side of the sprocket holes, so that they can be introduced upside down, generating nonsense.

The Teletypesetter code is an adaptation of the 5-level Baudot code, which has been used throughout the world in automatic telegraph and teleprinter (e.g., Teletype) equipment for many decades. Before 1970 the Baudot code is expected to give way to an international standard 7-bit code, represented in a standard 1-inch 8-level perforated tape, as recommended by the Research and Engineering Council of the Graphic Arts Industry.

Of the 64 unique codes in Teletypesetter tape, four codes are generally used as "precedence codes" to affect the operational meaning of codes that succeed them, as follows:

Shift (for upper case)

Unshift (for lower case)

Upper Rail (boldface or italic)

Lower Rail (Roman)

There are 19 other spacing, printing. format and machine control codes which are unaffected by the precedence codes, so that the available set of characters which can be set in type is 166 .

Some systems, used in local service, or by prearranged agreement between the sender and the recipient of transmitted tapes, sacrifice the use of two available codes (paper feed and quad right) in order to gain two additional precedence codes, called Upper Magazine and Lower Magazine. The
Mergenthaler Linomatic is an example. This allows a hot metal machine, such as Linotype, to mix two different fonts. Quite complex counting logic is required to keep track of the different character widths in the two fonts, and still provide justified copy, unless the two fonts are compatible in widths.

The complete TTS code is shown in Figure 19. It includes upper and lower case alphabetic characters, numerals, punctuation symbols, and all of the control functions necessary for hot metal linecasting machines. Where three symbols are shown for a given code, this means that a choice can be made between two different standard TTS codes in common use. Either fractions $1 / 8$ to $7 / 8$ are available for printing stock market tables, or the ligatures (ff. fi. fl. ffi. and ffl) are available for printing of text. The question mark and ampersand are available in both sets, but with different code patterns.

lt has been claimed that the introduction of tapeoperated line-casting has driven the "first significant wedge between the mental and mechanical functions involved in typesetting." 18 In addition, this type of equipment offers production advantages in the printing shop itself. Thus, "the impulses actuating the keyboard are uniformly timed causing the mats to be assembled with less chance of piling up, thereby enabling the machine to be operated at a faster number of slugs per minute." 19

\subsubsection{Tape Perforators With Rilbon Impression Printers}

Tape perforators without associated printing devices for producing hard copy, and intended primarily for the mechanical control of hot-metal casting machines, have been noted. However, keyboarding devices for the simultaneous produc. tion of both readily legible copy and machine-usable paper tape serve several major purposes: These include:

(1) Since the tape is machine-usable, it is also machine-re-usable, e.g., for the merging of variable address information with common message or text corpus and especially for the insertion of editing instructions and corrections to previously composed text.

(2) Humanly-legible copy is desired for on-line error-correction control and for proofreading generally: "The original 'Monotype' system, and teletypesetter systems, all placed reliance on the operator being able to 'read' the codes on the tape, i.e., to examine and understand them, but as codes become more complex and systems of alteration and merging faster and more automatic it is better to provide a "plain' language output." 20 Furthermore, in photocomposition systems, problems of page revi-

\footnotetext{
1x "Computerized Typesetting." 1964. p. 1.

${ }^{14}$ Heffman. 1959. p. 192

a) Duncan. 1964. p. 124.
} 
sion and insertion of corrections are severe, therefore, ". . . hard copy is becoming more essential in order to produce clean tape to put into a phototypesetting unit. ..."21

(3) A single keyboarding operation provides both control information and material for multipurpose use. Examples involving paper-tape production of library catalog card information and of even more comprehensive systems envisioning the complete cycle from original manuscript preparation through publication to automatic derivation of related bibliographic data will be discussed in a later section of this report.

Ribbon impression typewriters that can operate tape perforators include the following:

Friden Flexowriter

Friden LCC-S Justowriter

Friden Justowriter

Remington Rand Synchro-Tape

Smith Corona Typetronic

Mergenthaler Linofilm Keyboard

IBM 870 Document writing system

IBM 1050 Data Communication Terminal

Dura Mach-10

Invac TMP 200

Invac TMP 100

Greene Data Tape Letteriter

Soroban Tape Key Punch

Crosfield Roboset 18

Photon Keyboard

The machines listed above are basically electric office typewriters combined with paper tape perforators. In some cases the tape perforator is built into the frame of the typewriter and is driven by the same motor. In other cases, the tape perforator is external to the typewriter.

Communication teleprinter tape perforators are not included here because these machines have historically been monocase, printing upper case in the United States and England, and lower case in Germany and other parts of Europe. In a year or two communication teleprinters should be available in dual case versions with internationally standardized 7-bit codes and 8-level perforated tapes, the extra level allowing a parity bit to be included for each tape character.

The printer-tape perforators listed above can generally perforate the $7 / 8$ inch 6-level teletypesetter (TTS) code. They are also used with $7 / 8$ inch 7-level tape or 1 inch 8-level tape, but the tape codes are far from standardized, being computer codes or unique codes. The Letteriter is a pneumatically operated machine having a code not used in any other equipment. The Linofilm perforates a 15-level tape, for typesetting purposes only, with each tape character containing information on the character and its font, size and width, or a machine control function.

\footnotetext{
?t H. P. Whittaker, remarks, Computer Typesetting Conference Proceedings. 1965, p. 171.
}

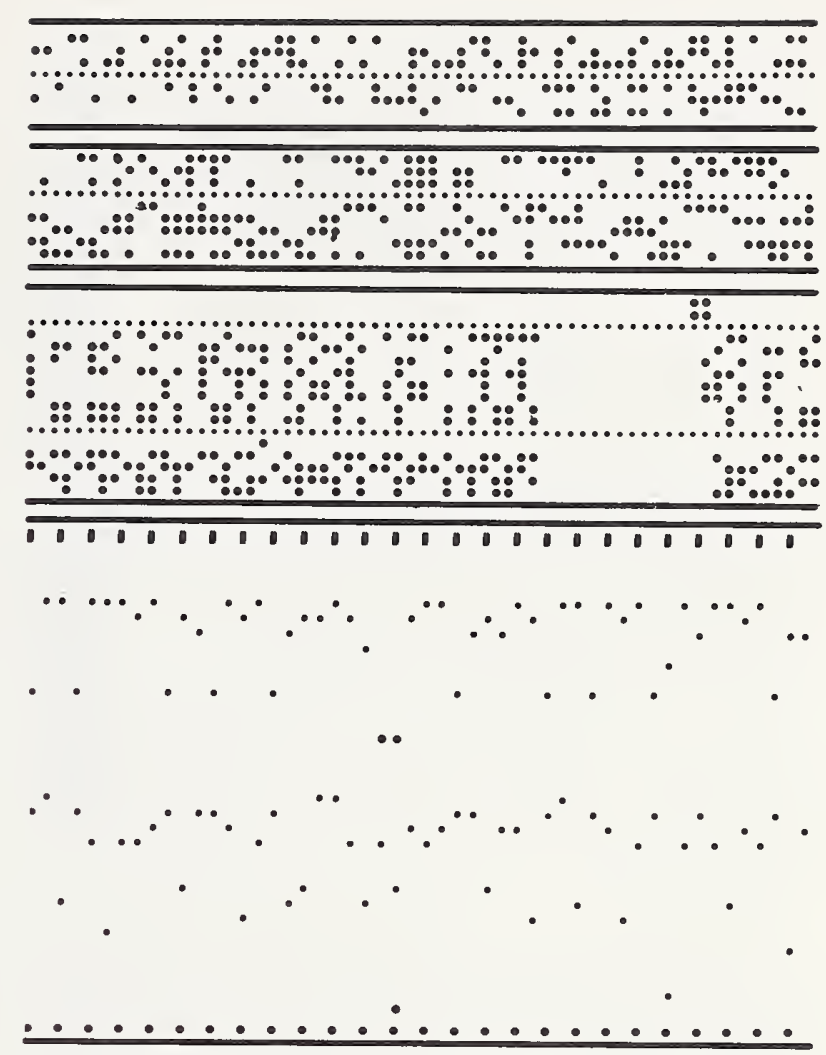

Figure 20. Perforated tapes used in typesetting.

Figure 20 illustrates some of the perforated paper tapes currently used for typesetting, taken from production samples, including an example of at least one keystroking error.

\subsection{Photocomposition Equipment}

The introduction of photocomposition techniques has been described as the third major development, following movable type and hot metal casting, to change the structure of the printing industry. ${ }^{22}$ "The classical method of 'setting type' as a preliminary to actual printing operations has been supplemented during the last two decades by "photocomposition.' In this latter process, images of the successive characters that are to appear on a printed page are projected by an optical system onto successive portions of a sheet of photosensitive material. This is developed and used as the basis of offset or letterpress reproduction." 23

As we have seen, in the chronological outline given earlier, from the Photon (Lumitype) work of about 1945 onward and the introduction of computer programs to drive photocomposers (IBMMergenthaler and Bozman for the Linofilm, Barnett for Photon), a number of techniques and devices

\footnotetext{
22."Photutypesetting Picks Up the Pace," Business Week, Dec, 14, 1963, p. 158.

${ }^{2}$ Barnett et al., 1962, p. 59.
} 
have occurred up to the more recent developments involving the use of electronic character generators in photocomposition systems. Deferring discussion of the latter developments, we will first consider briefly the principles underlying photocomposition techniques and then discuss classes of available and proposed photocomposer devices and systems.

\subsubsection{Techniques of Photocomposition}

Photocomposition involves the transfer of optical characters from a negative master onto a photowaphic emulsion, with provision for proper illumination. image formation, magnification or reduction, and positioning of each image. Many techniques and configurations of mechanical, optical and electronic equipment have been devised to accomplish photocomposition.

These are "machines, which, typically, photograph letters and numbers and produce pictures of columns of type. These pictures can then be pasted into a page layout, which is photographed again. The resulting negative is used to make a photoengraving of the page that is used in the actual printing." ${ }^{24}$

Typographic quality output is achieved in photocomposition by automatic selection of the right one of relatively large sets of characters, by point size adjustments including those necessary for sub. scripts and superscripts, by controlled positioning, and by insertion of special symbols and characters.

Thus, for example, as described by Barnett, Kelly, and Bailey: "In photocomposition . . . successive characters along a line are displayed with collinear base lines, regardless of their magnification (i.e.) point size. . . The same character is displayed in different point sizes, in photocomposition, by the use of different lenses. ... Special characters can appear anywhere in a text, and can be magnified and spaced in the same ways as ordinary alphabetic characters." 25

The generation, accurate placement, and permanent storage of hundreds or thousands of highquality symbols per second demand that opticalphotographic techniques be employed, since the speeds are too great to permit mechanical symbol selection. Photography on film or paper seems to offer the preferred recording method, although other graphic storage techniques, such as electrostatic, thermographic, thermoplastic, photochromic or video signals on magnetic tape offer alternate recording possibilities. However, sincc all photocomposers existing or under development appear to use photographic recording, we can start at the light-sensitive emulsion, and explore the various methods used to form and position the optical images.

High-speed photocomposers may be classed broadly into six principal categories:

a. Those which have a stationary master glass

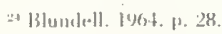

2s Barnett et al. 1962. p. 60)-633. grid from which the symbols are selected, sized and positioned onto the photographic emulsion by purely optical-mechanical means. An example of this is the Mergenthaler Linofilm System.

b. Those which have the symbols on a spinning master glass grid and a single stroboscopic flash tube that can be flashed at the proper instant to select any. desired character, with size control, imaging and positioning by optical-mechanical means. Examples of this are the Photon 200, 500 , and 700 series machines and the HarrisIntertype spinning disc photocomposer.

c. Those which have the symbols on a stationary master glass grid, with a separate flash tube for each symbol, a reciprocating lens which positions all symbols across a complete line through an optical tunnel, with the various flash tubes being fired as each symbol is imaged in each of its desired positions in the line, permitting a complete line to be composed in one pass of the lens. The only machine of this type is the Photon 900 (Zip or GRACE) machine, the first of which was delivered to the National Library of Medicine in 1964.

d. Those which have the symbols on a stationary master glass grid, steadily illuminated and optically projected onto a light-sensitive cathode of an image-dissecting tube, which simultaneously produces scanned video electron beams from all symbols, one of which is allowed to pass through an electrostatic wire grid selection matrix to produce a video brightening signal for a high-resolution cathode ray display tube, with positioning and size on the display tube being controlled electronically. The only such machine of this type is the Mergenthaler-CBS Linotron system being developed for delivery to the Government Printing Office and the Air Force in 1967, employing the CBS "Reconotron" image dissector-scanner, and the CBS "Microspot" high-resolution cathode-ray display tube.

e. A special cathode-ray tube employing a beam-shaping metal plate perforated with the symbol shapes, with means to cause the electron beam to impinge on any symbol area, emerging as a shaped electron beam, which can be deflected to display the symbol at any position on the face of the cathode-ray tube. An example is the Charactron tube, employed in photocomposers built by the Stromberg Carlson Division of the General Dynamics Corp., such as the S-C 4020. The repertoire of symbols is usually severely limited to less than 100.

f. Methods employing a conventional or highresolution cathode-ray tube to display the symbols as they are generated by some means which will produce suitable beam deflections and video brightening to form the symbols.

\subsubsection{Classes of Photocomposers}

There are many simple keyboard or manual dial operated photocomposers for making heading and 


\begin{tabular}{|c|c|c|c|}
\hline & & & \\
\hline $101 / 1011100$ & 19 Typography for & 30 typogRaph & 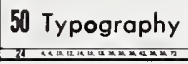 \\
\hline 11 Typog & 20 Typography $\mathrm{f}$ & 31 Iypagrap & 51 Typography f \\
\hline 13в Ц॥" 21:ヨ & 21 Typograpby fo & 33 Typograp & 52 Typography \\
\hline 13 TYPOGRAP & 23 Typography $f$ & 34 Typography $f$ & 53 Typography \\
\hline 16 Typograph & 24 Iyposeaphy forens : & 36 Typography & 54 Typograp \\
\hline 17 Typography & 25 Typography for & 37 Typography $f$ & 55 Typog \\
\hline \multirow[t]{4}{*}{ 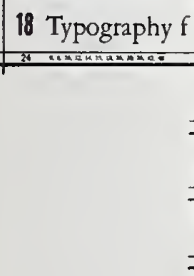 } & 26 TYPOGRAPHY FO & 38 7ypagraphy 6 & 56 Iypogrophy to \\
\hline & 27 TYPOGRAPH & 45 Peas....6 $6 \mathrm{~ms}=\mathrm{s} 100$ & 60 Typography for \\
\hline & 28 Iypagraphy to & $\begin{array}{l}48 \text { Typogrophy forms } \\
10 \times 10\end{array}$ & 61 Typography f \\
\hline & 29 Typography f & 49 Typography fo & 62 Typograp \\
\hline
\end{tabular}

FIt,URE 21. Sample Output, Varityper Series 800 Photocomposing machines.

title strips. Examples of such machines are the Filmotype, Headliner, Typro, Hadego, Foto-Riter, Protype, Photo Typositor, and Strip Printer. These are machines which "use the principle of exposing individual characters one at a time from a master pattern on a strip of photosensitive paper which can then be simply developed." 26 For example, the Dutch phototypesetting machine, Hadego, involves manual assembly of character matrices (white on a black plastic material) into a special composing stick and setting of the desired enlargement factor with a range of sizes from 4 through 82 point. $^{27}$ Samples of some of the character set, type size, makeup and display advantages of such devices are illustrated in Figures 21 and 22.

Traditional hot metal typecasting machines have also been modified into photocomposing machines. Examples are the Fotosetter, Fotomatic and Monophoto. Concurrently with these evolutionary modifications of hot metal machines into photocomposers, there has been a steady development of freshly-designed photocomposing machines, permitting greater flexibility and a breakthrough in the composing speed barrier imposed by the mechanical symbol selection necessary in typecasting.

Table 1 shows the range in speed attainable from various American-built photocomposers.

In 1964, the Lanston Monotype Company introduced the "Monophoto," an adaptation of the Monotype hot metal typecaster. Monophoto utilizes a separate punched paper tape keyboard, which is a slightly modified version of the Monotype keyboard. The output from the keyboard is a 31

${ }^{26}$ Hoffman, 1959 , p. 196.

27 "Filmsetting and Cold Type Methods," 1964, p. 8.

\begin{tabular}{|c|c|c|c|c|}
\hline SCRIPTS & a. & $d_{a}$ & $t_{a}$ & Ota \\
\hline CALLIGRAPHIC STYLES & $\mathrm{Aa}$ & Ala & $\mathrm{Aa}$ & $c A a$ \\
\hline CONTEMPORARY CASUALS & $\mathrm{Aa}$ & $\mathrm{Aa}$ & Aa & $\mathrm{Aa}$ \\
\hline FREE STYLES \& NOVELTIES & A $\mathbf{A}$ & $A a$ & Aa & $A a$ \\
\hline ROMANS & $\mathrm{Aa}$ & Aa & $A a$ & Aa \\
\hline SANS SERIFS & Aa & $\mathrm{Aa}$ & Aa & Ad \\
\hline SQUARE SERIFS & $\AA_{8}$ & Aa & $\overline{A a}$ & Aa \\
\hline EXTENDEDS & $5 a$ & Aa & Aa & 4 \\
\hline
\end{tabular}

$\begin{array}{ll}18 \text { Point } & \text { ABCDEFGHIJKLMNOPQRSTUVWXYZ., } \\ \text { FUTURA abcdefghijkImnopqrsfurwxyz \& I? } \\ \text { Demibold } 12345678901234567890 \$ \$ \% \% / ., i^{\prime \prime \prime \prime}-() \ldots{ }^{* . .}\end{array}$

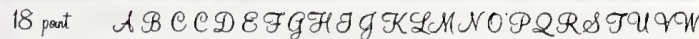

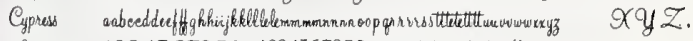
Seript $12345678901234567890 \mathrm{sc \%} / \ldots \cdots, \ldots$ ()..." \&!?

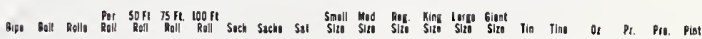

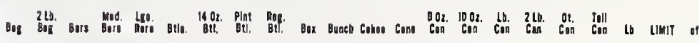

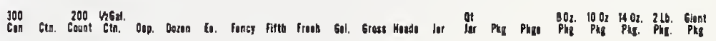

Figure 22. Sample Output, Friden Typro.

TABLE 1. Speeds of Automated Photocomposers

\begin{tabular}{|c|c|}
\hline & $\begin{array}{l}\text { Theoretical Text } \\
\text { Composing Speed } \\
\text { Characters/Secon }\end{array}$ \\
\hline \multicolumn{2}{|l|}{ Photocomposer } \\
\hline Lanston Monophoto.. & 3 \\
\hline ATF Typesetter.................... & $5-6$ \\
\hline 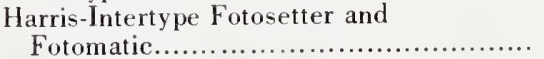 & $6-8$ \\
\hline Photon Spinning Disc Series 200 & \\
\hline 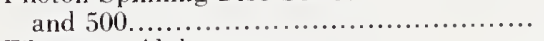 & $8-10$ \\
\hline Filmotype Alphatype................ & $9-11$ \\
\hline Mergenthaler Linofilm............... & $10-18$ \\
\hline Harris-Intertype Spinning Disc............... & 20 \\
\hline Photon Spinning Disc Series $700 \ldots \ldots \ldots \ldots . . .$. & 22 \\
\hline Photon Series 900 (ZIP or GRACE)....... & $300-500$ \\
\hline 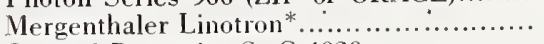 & $1,000-10,000$ \\
\hline General Dynamics S-C $4020 \ldots \ldots \ldots \ldots \ldots$ & $12,500-17.400 * *$ \\
\hline Benson Lehner Transdata.... & 62,500 \\
\hline
\end{tabular}

*First delivery to the GPO in late 1967.

**Repertoire limited to 64 or 88 symbols.

channel paper tape which is used to drive the photo unit.

The photo unit is similar in physical design and in operating characteristics to the Monotype leadcaster. The film negatives are mounted in individual single character matrices and are stored in a matrix case with room for 255 individual matrices (characters) as in standard Monotype systems.

The optical system (diagramed in Fig. 23) utilizes a 40-watt light source which is directed through a condensing lens, through the die case (particular character), through two right-angle prisms, through a magnification lens and then onto a single piece of sheet film which has been taped onto a drum. 


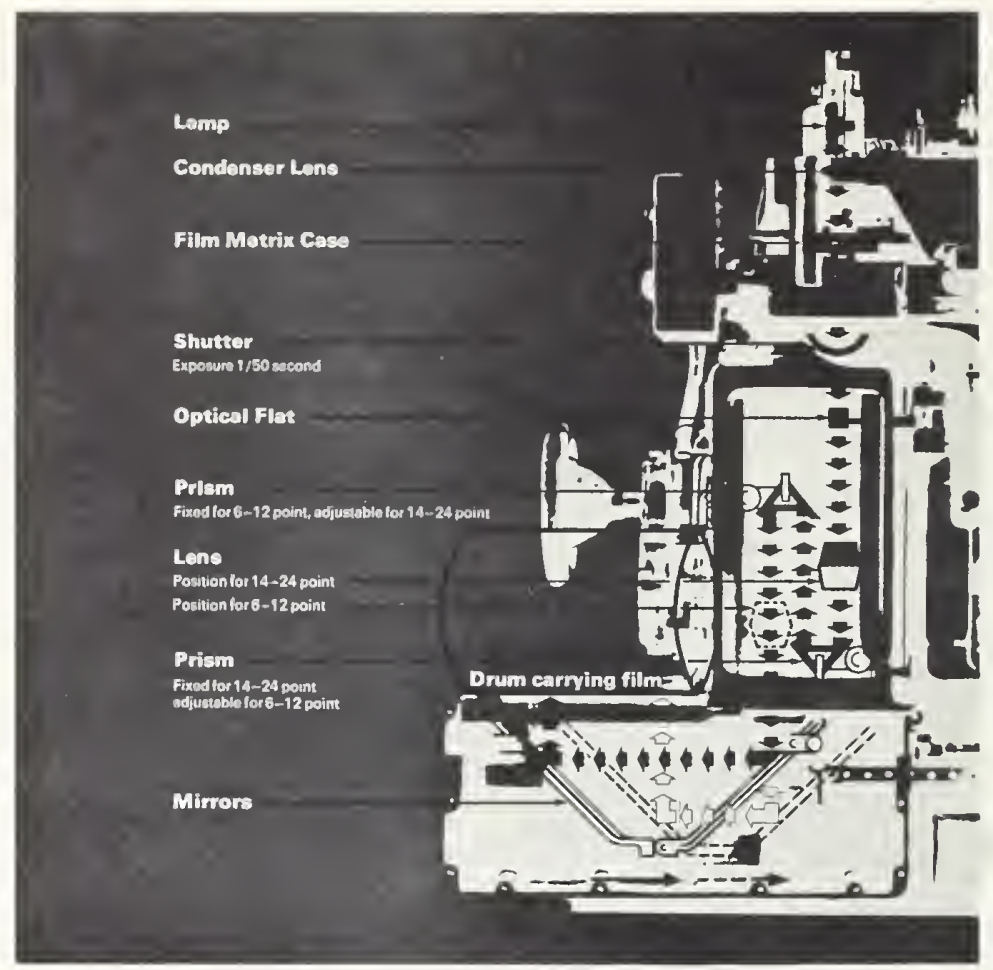

Figure 23. Monophoto Optical System.

Changes in point size are accomplished by changing the position of the lens. Corresponding changes in spacings between letters and set sizes are accomplished by moving a gear shift lever. Leading (vertical spacing between lines) is variable within a limited range. The effective speed of the tape operated photo unit is three characters per second.

Although this machine is apparently the slowest of the available photocomposers, it has been pointed out that "the chief virtue of the "Monophoto" has been that it has acted as a bridge between hot metal casting and photocomposition and has given works managers sufficient experience of film correction techniques to know that they are better avoided." 28

The ATF Typesetter (Figure 17), made by the American Type Founders, Inc., consists of two units, a keyboard unit and a photo unit. Both units have a keyboard and perforated tape reader. The keyboard unit is basically a Flexowriter which has been modified by the inclusion of width card circuits for indication of justification range plus the addition of special functions. The keyboard does not punch the width information for each character into the tape. The photographic unit has identical width cards from which the width information is obtained. The photo unit has an optical matrix disc that holds 168 characters. limiting the type mixing ability to two fonts. Different point sizes are obtained by using different discs, but this requires manual changing of discs. The dise is held stationary for each exposure, and is controlled by an 8-level perforated tape from a computer or from the keyboard unit, or it can be controlled by the keyboard on the photo unit without the use of perforated tape.

The speed of the basic model is about 5-6 characters per second. Duncan notes that the ATF typesetter (Model B) uses a disc with 3 sets of 168 characters and that these models retain "the justowriter's ability to edit on the job by stopping composition at any time and overriding the tape with a manual keyboarding ... [and] to read the justification and instruction codes before setting the line even though these codes occur later in the tape than the character codes being set." 29

In 1949, a modification of the Intertype linecasting machine was made commercially available. The modification was such that each matrix had its casting mold replaced by a window surrounding an optical master of the symbol represented by that matrix. The matrices are selected one-by-one and assembled into a line, as in line-casting, but instead of casting a line, the line of symbols is photographed. Then the matrices are automatically returned to their storage magazines, as in line-casting, and a new line is composed. This machine is known as the "Fotosetter" (Figure 24). The Fotosetter fonts are contained in individual 


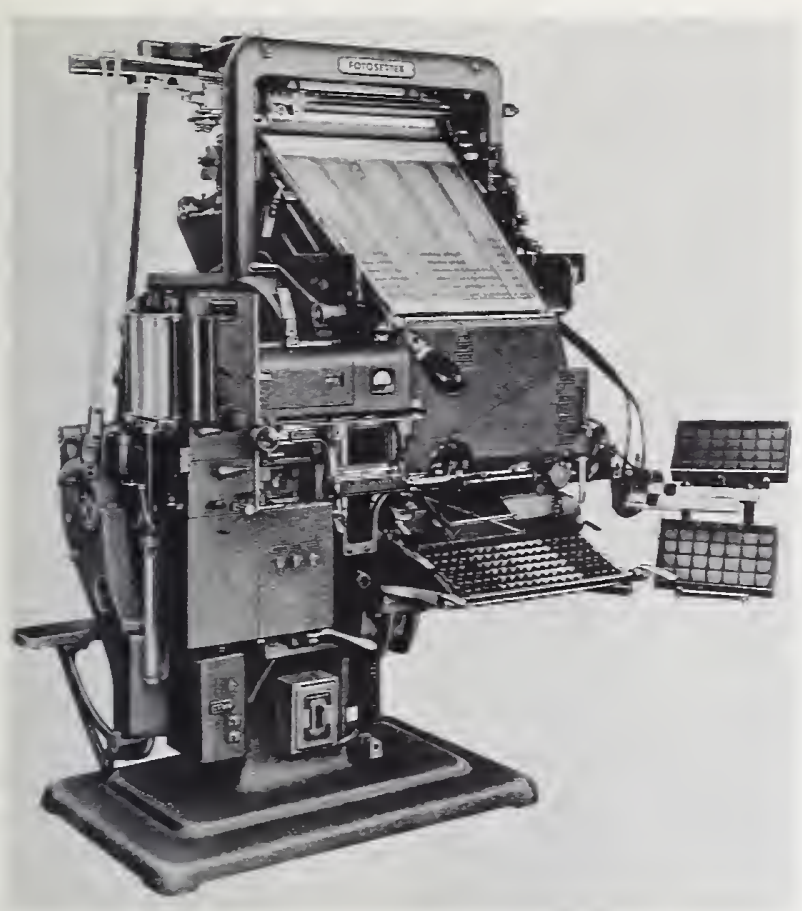

FiglRE 24. Intertype Fotosetter.

single-character matrices in the form of the trans. parent images and are stored in four magazines having 117 channels each. It is necessary to maintain a supply of each character. The Fotosetter does have a lens system. however, which eliminates the need for matrices in different point sizes. That is, "by exposure to a turret-type camera the images are photographed in a number of possible sizes: the result is a film that is extremely sharp." 30 The Fotosetter is widely used in book publishing and operates at $6-8$ characters per second.

In 1964 Intertype introduced the "Fotomatic" machine which can use upper and lower rail dualletter matrices, or the single-letter matrices of the Fotosetter.

Photon, Inc., of Wilmington, Massachusetts, originated a new class of photocomposer (following the inventions of Moyroud and Higgonet). having the master matrix of characters on a spinning disc, as shown in Figure 25, with an intense strobe light accurately timed to flash as the desired character comes into position. A mechanically-operated lens and prism system controls the size and placement of each character onto a roll of photographic film.

The matrix discs of Photon machines provide for sets of 1440 characters which are etched onto eight concentric annuli on a disc of opaque material and standard size. Many different Photon discs have been designed and, in addition, as in use by the American Mathematical Society, Pi-mat attach-

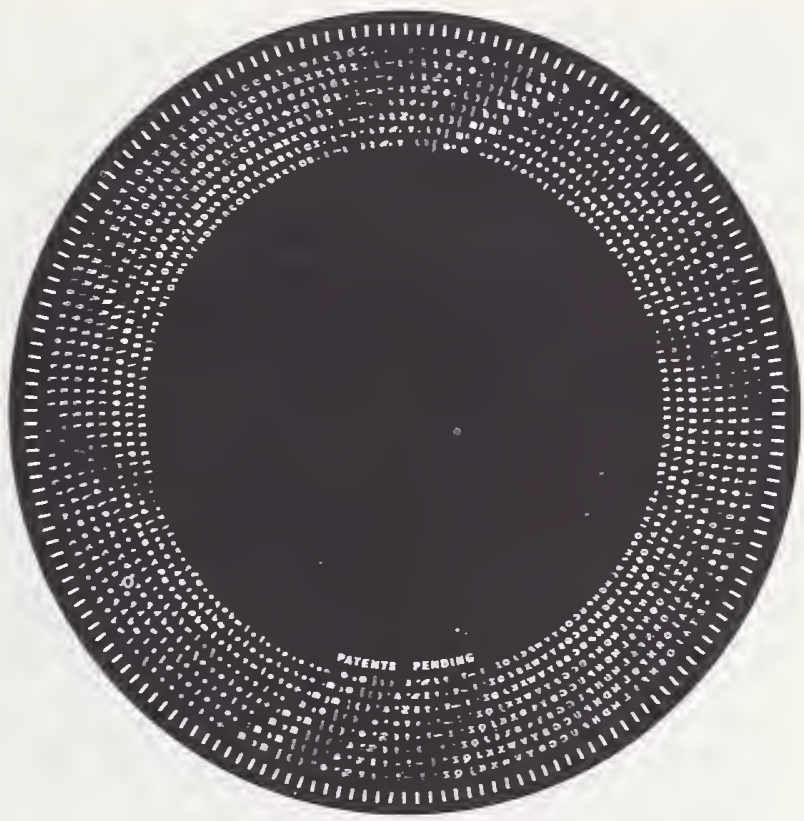

Figure 25. Photon disc.

\section{SERIES 540 TAPEMASTER}
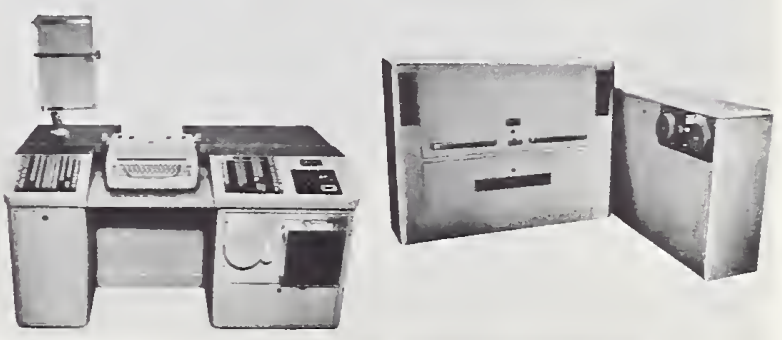

JUST A FEW ADVANTAGES OF THE PHOTON 540 TAPEMASTER

PHOTON 540 FEATURES

Here for the first time is a typesetting technique that enables you to produce galley after galley of letter-perfect typography - with little more effort than it takes to type a page of copy!

Here is a type composing technique that permits you to mix type sizes and faces instantaneously, 10 correct errors without "killing" lines... to turn out razor sharp area composition.

Here is a completely self-contained "composing room" ... a compact task force of typesetting equipment that offers and delivers unlimited versatility ... unparalleled economies.

The 540 Tapemaster-for peak efficiency - consists of a number of tape-perforating keyboards, a single photographic unit and a control unit with tape reader. This system allows full eapacity operation and permies the printer to double or even triple his output with a minimum outlay of capital.

$$
\begin{aligned}
& \text { - } 10 \text { characters-a-second output } \\
& \text { from tape } \\
& \text { - Unlimited mixing of style and size } \\
& \text { in the same line } \\
& \text { - } 16 \text { typefaces - } 12 \text { sizes } 5 \text { point } \\
& \text { to } 72 \text { point } \\
& \text { - Fingertip access to at least } 192 \\
& \text { type fonts } \\
& \text { - Length of all line serting in pica } \\
& \text { measures } \\
& \text { - Line lengths up to } 42 \text { picas } \\
& \text { - Corrections wrthout "killing" whole } \\
& \text { lines } \\
& \text { - Automatic line justrication of mixed } \\
& \text { type } \\
& \text { - Automatic quadding, leaders. } \\
& \text { centering }
\end{aligned}
$$

CONSOLIDATION OF THE COMPOSING ROOM OPERATION

PHOTON Wilmington, Massachusetts

Ficure 26. Photon Series 540 Tapemaster. 


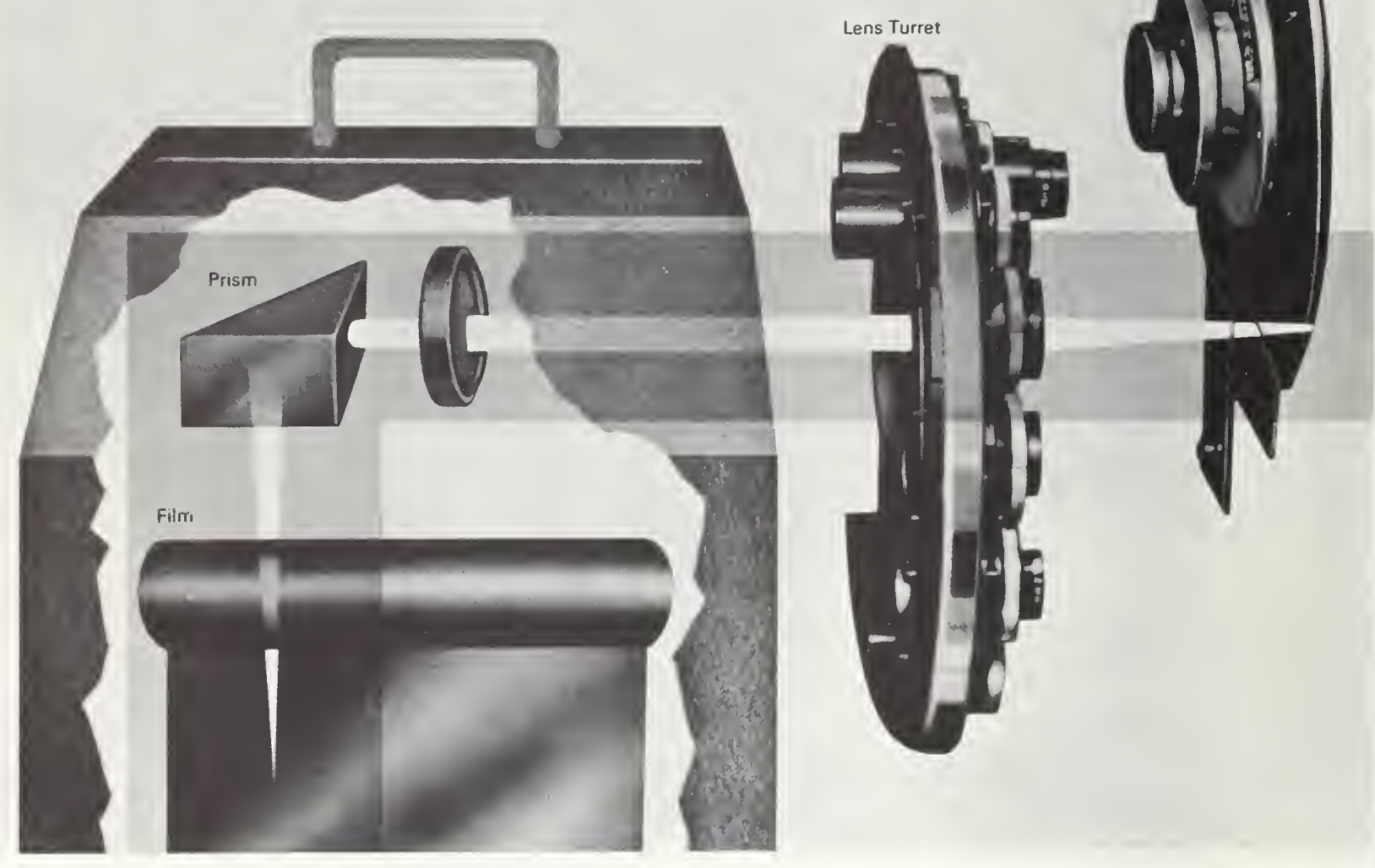

Figure 27. Photon photowraphic system.

ments can be provided, consisting of an auxiliary disc which contains up to 8 interchangeable characters. The optics arrangement is such that any one of these $\mathrm{Pi}$-mat positions can be reached by keyboarding.

"When the machine is in operation, the disc rotates at a high speed about a fixed axis. During each revolution of the disc, a light beam may be projected along a fixed path, through one of the annuli on the disc that is etched with characters. The illumination persists for a time interval that is very short compared with the period of rotation of the disc. An almost static image of any single character on the disc thus can be produced for a very short time interval during each rotation. The timing of the burst of illumination and the choice of annulus determine which character is displayed during any rotation." 31 Switching between annuli, as required, is accomplished by mechanical displacement of the disc axis.

The Photon Model 200 is operable either by keyboard or from paper tape. The members of the

\footnotetext{
3 Barnetl el al., 1962, p. 59.
}

newer 500 series such as the 540 , shown in Figure 26, were designed specifically for tape operation and therefore separate the keyboard from the photo unit.

The Photon can mix 16 different type styles of 90 characters each in 12 different point sizes ranging from 5 point to 92 point for a total of 17,280 characters available at the keyboard without changing magazines or fonts. When a character to be photographed is selected, a stroboscopic flash of light (four millionths of a second) optically floods the corresponding character at the proper instant and exposes its image through appropriate lenses onto photographic film or paper. One character can be exposed at each revolution of the disc. The speed of the disc which is 8 to 10 revolutions per second is therefore a determining factor in the composing speed of these Photon models.

The variable escapement unit carries the prism shown in Figure 27 which directs the beam of light onto the film. This is a mechanical movement which could not be too effectively speeded up without creating mechanical problems due to mass 
and vibration. The leading unit drives the film take-up magazine thus providing the vertical spacing between lines. It can provide spacing from .1 point to 49.9 points for each leading code and will operate in the forward or reverse direction.

The perforated tape that drives Photon models constructed prior to the magnetic tape machines (GRACE and ZIP) contains in coded form the specifications for the appropriate track (half an annulus) on which the desired character is located, the sequence number of the position of the desired character along the track, the lens setting which determines the magnification or point size factor and, in most models, a precise width or escapement code which sets the distance required between the leftmost edge of the desired character and that of the character to follow.

An exception to the latter case is the Photon 513 (as used, for example, with the RCA 301 computer at Perry publications). The 513 uses only a single code for each character together with information as to line space deficit and hence the word spaces required for justification. "The 513 accepts a 6-level code without information as to widths of the characters. Therefore, the width has to be re-generated in the machine and justification has to be re-calculated." 32

The Photon 500 series, in general, provides various tape-driven units which operate from 6-, 7-, or 8-bit (channel) perforated tape. The model

32 R. A. Higgonet, remarks, Computer Typesetting Conference "Proceedings," 1965 , p. 223.
560 (as used by Barnett, for example) operates with 8-channel tape. The character code for 560 operation has two components: the character identification code itself and the precise escapement code which is the relative width value for the character. Operating speed for actual typesetting in 560 systems is at 8 characters per second.

The total number of bits required is 16 , however the Photon accepts this as two successive 8 bit codes. The keyboard unit produces the character identification in standard 8-level paper tape. (Photon will provide a special tape reader and translator for any of the various codes which employ 8-level tape.) The relative width identification is added by the photo unit by the use of printed circuit width cards for each type font. It should be noted that Photon is now offering photo units which will accept standard Monotype tapes (3l channel) and standard TTS tapes (6 channel).

In 1964 the Harris-Intertype Corporation introduced a spinning disc photocomposer, similar in principle to the Photon spinning disc machines, having a strobe flash of one microsecond, and a disc speed capable of yielding 20 characters per second (Figures 28-30).

At the "Westprint 1964" exposition held in Los Angeles June 7-13, 1964, this equipment was first publicly demonstrated in operation along with the Intertype Monarch typesetting machine. The Intertype disc is of glass inscribed with 480 different characters. Nineteen different point sizes, from 5 to 72 , can be accommodated. Output is to either film or photographic paper.

\section{HARRIS-INTERTYPE ELECTRONIC PHOTOGRAPHIC TYPESETTING SYSTEM}

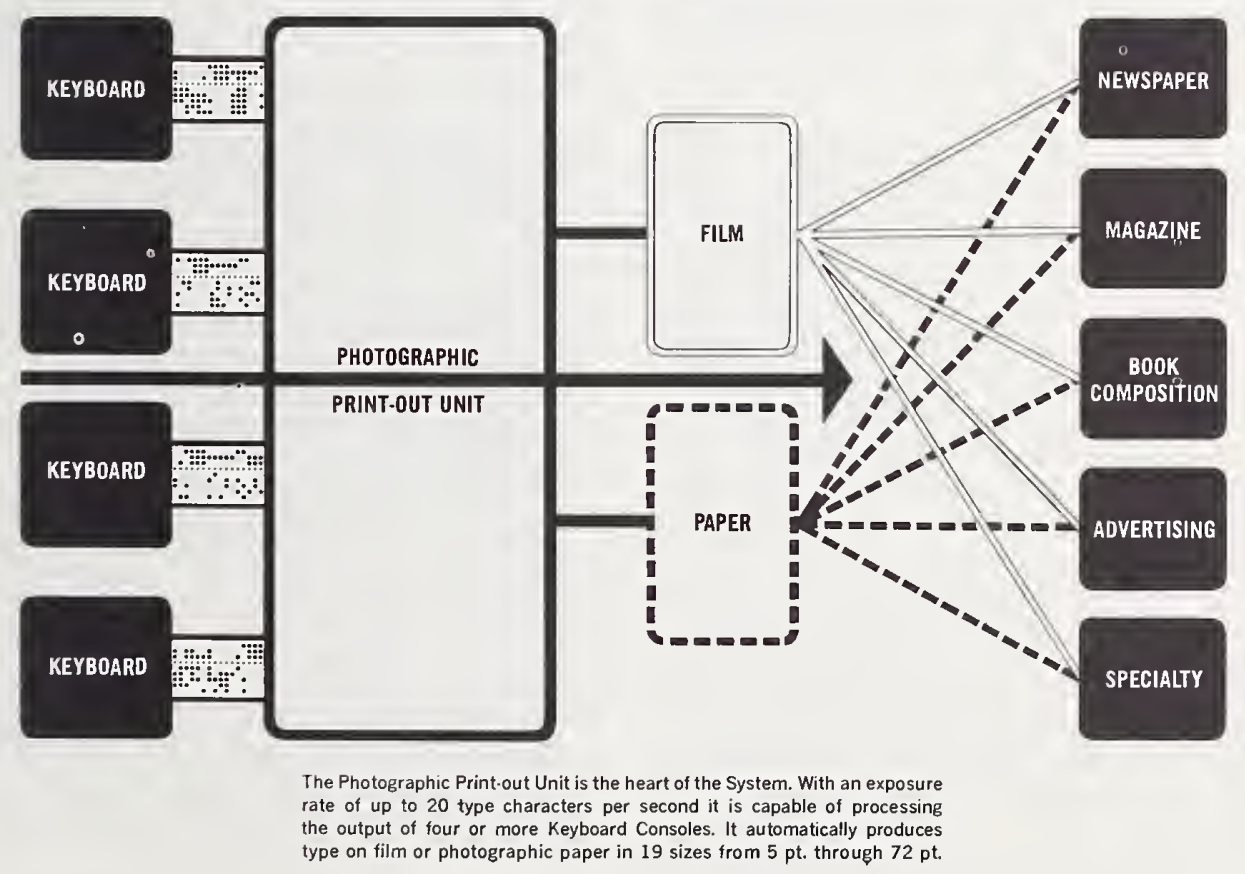

FIGURE 28. Harris-Intertype system. 


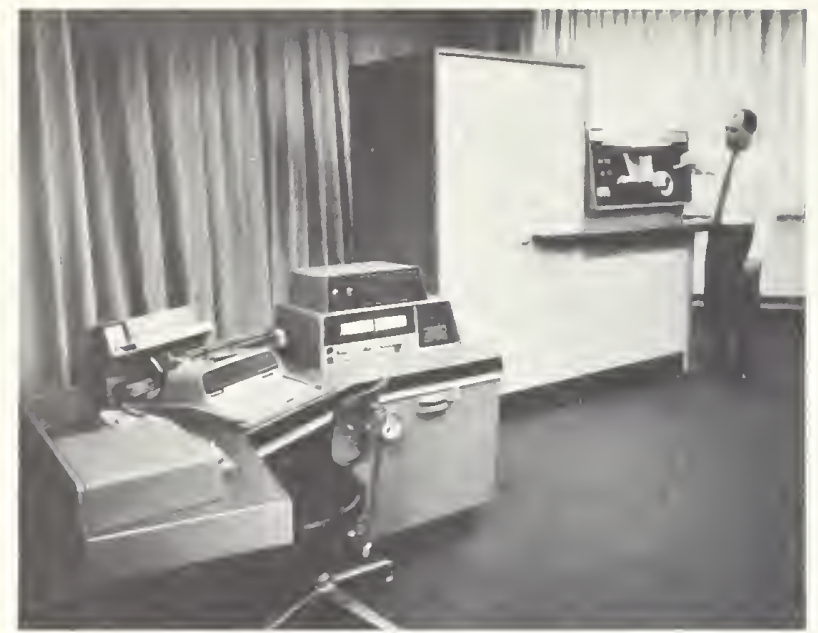

Ficiune 29. Harris-Intertype installation.

The system is composed of two basic units - one, a keyboard used to prepare copy in the form of perforated tape (6-, 7-, and 8-channels) to activate either hot-metal or photocomposition machines, and the other, a relatively high-speed photocompositor unit.

Built-in justification-hyphenation techniques involve the use of various combinations of manual monitoring (Series 100), logical rules (Series 200) and logical rules plus an exception dictionary of up to 10,000 words.

Six-channel tape inputs enable use of four fonts intermixed, and the 7-bit code keyboard allows up to eight fonts to be processed and mixed. It is also possible to accept wire service copy and to rejustify and rehyphenate as necessary. Prior to the 1964. Westprint demonstration, prototype installations were field tested at Graphic Services, lnc., for book composition applications and at the South Bend Tribune.

In quick competition, the Linofilm system was introduced by Mergenthaler Linotype Co., after the appearance of the Photon spinning disc machines. In Linofilm, the master characters are stored on glass grids, one of which is shown in Figure 31. There are 88 symbols on a grid, corresponding to the 88 symbols on an electric typewriter. Eighteen of these grids are stored in a turret, as shown in Figure 32. Choice between these 18 grid fonts is under tape-code control.

Thus 1,584 characters are available under tape control, and additional grids can be substituted manually if needed.

For each type style, there are usually three different grid fonts of 88 characters to give proper proportionality to the characters. The font series are designated A, B, and C. for small, medium, and large point sizes. It therefore uses three different size fonts, each covering a separate portion of the total range of point sizes.

In the Linofilm system, a selected grid font is stationary at the time the character is being photographed. A flash tube exposes the entire 88-

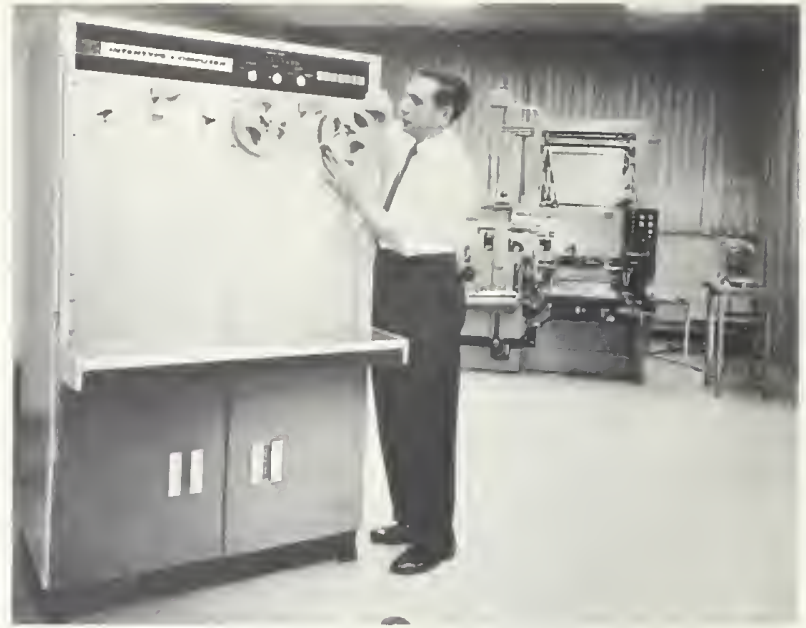

Ficure 30. Harris-Intertype equipment.

character font, and a shutter system consisting of a series of eight shutters masks out all but the one character called for. A series of 88 "lenslets" carry the light to a collimator which projects the character in the proper plane. At this point, the image is magnified by a pair of lenses mounted on a sliding bar which provides the variable magnification. From here the image is redirected by a front surfaced mirror to the film plane. The mirror is mounted on a sliding bar which moves across the page as the line is being exposed.

The Linofilm system consists basically of a keyboard unit and a photo unit. Its keyboard unit

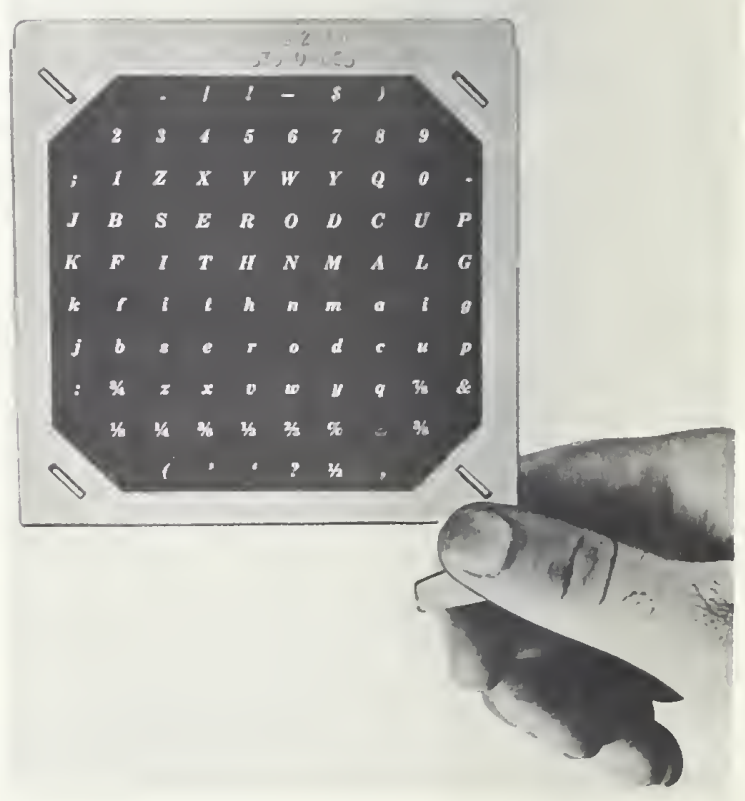

FI,URE 31. Linofilm grid font. 


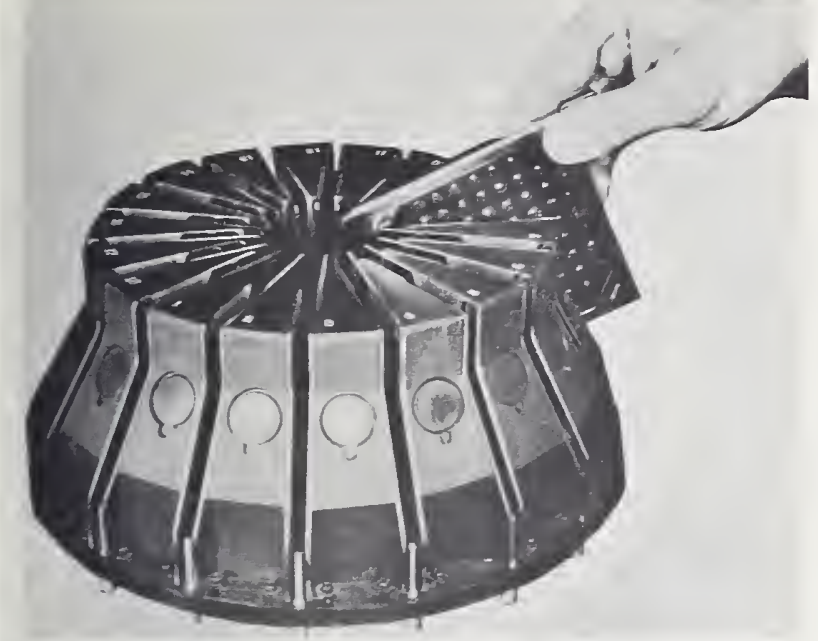

Figure 32. Linofilm wrid font turret.

produces a 15-channel tape which includes character identification and width values.

The photo unit operates from the 15-channel paper tape and automatically changes font and type size and composes onto film at a maximum speed of from 10 to 18 characters per second. Additional time is used up by font changes, lens changes, and the interlock between lines. The output from the Linofilm photo unit is positive film or paper which is processed in a darkroom by photographic processing methods.

Mergenthaler has introduced a Linofilm converter which will convert magnetic tape produced on a computer into 15-channel Linofilm paper tape. Nergenthaler has also introduced the Linofilm "Quick," a photo unit that operates directly from 6-channel Teletypesetter perforated tape.

A pioneering example of the use of a Linofilm photo unit in conjunction with a large general purpose computer was that of the Russian translation project at the IBM Thomas Watson Research Center at Yorktown Heights, New York. Here it was necessary to provide buffering to step down the high speed of magnetic tape (200 microseconds per character as computer output) to that acceptable to the photocomposer (20 milliseconds per character). A set of relays replaced the normal paper tape input action. ${ }^{33}$ This was also the equipment used in Bozman's first work.

The Linofilm system includes the Linofilm Composer which is used for positioning, and for enlarging or reducing any line to exactly the size required with no reference at all to conventional point sizes. This simplifies the work of the make-up man as all he need indicate to the keyboard operator is the type face and size that falls within the extremes of the finished job. The keyboard operator has a simplified job as well since he need not shift from

23 International Business Machines Corp., "Graphic Composing Techniques," 1962. p. $18-19$.

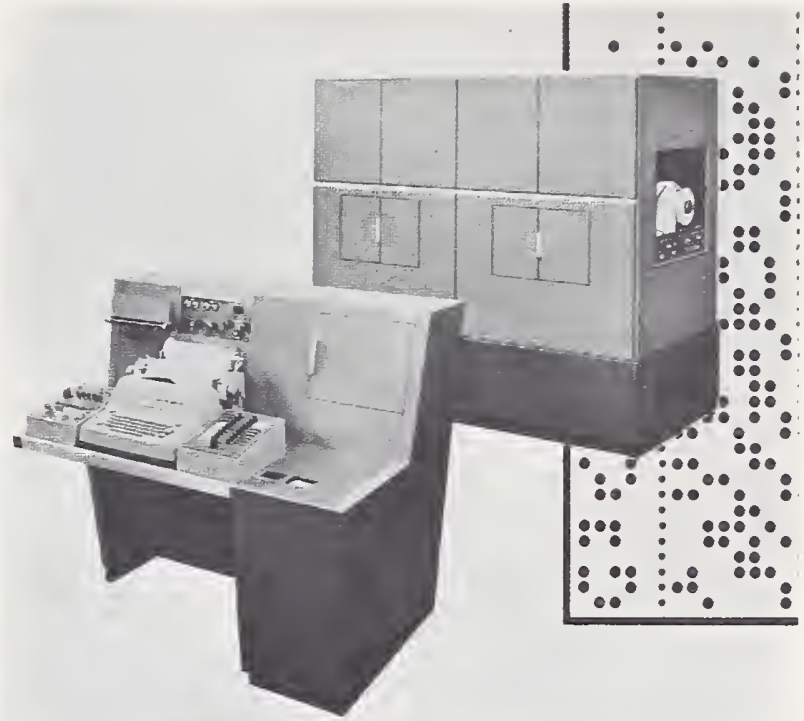

Figure 33. Linofilm system.

one size to another. (A complete Linofilm System is illustrated in Figure 33.)

The composer operator works from a layout on tracing paper which is placed over the screen of the composer. He loads the exposed and processed film from the photo unit into the composer, and by manipulating four simple controls, he can take any type up to 36 point from the photo unit, reduce it two-thirds, or enlarge it to six times: its original size - a range of from 4 to 216 points. He can set lines at any angle up to $90^{\circ}$.

Duncan reports that "the keyboard has recently been modified to include mixing facilities ('Linamix') and editing simplified by a tape merging and reproofing device ('Tape Combiner')." 34

As in the case of the Photon spinning disc machines and other disc-type photocomposers, speed is essentially limited by the mechanical system. "In Linofilm, the shutter, magnification system, and escapement system all have mechanically moving parts with considerable mass to overcome." 35 Nevertheless, "the Linofilm is built like a battleship and produces a fine quality product." 36

The Alphatype, made by the Filmotype Corporation, is comprised of three units. The keyboard unit is an electric typewriter. The recorder unit records characters and controls on a special 10 channel magnetic tape, employing mixed frequencies on the continuously moving tape, which is started and stopped by means of a foot pedal. This tape is not compatible with any computer. A width card for each font provides 15 widths, from 4 to 18 units, for the different symbols. The character width and line justification information are generated by the recorder unit and recorded on the magnetic tape.

\footnotetext{
${ }^{4}$ Duncan, 1964, p. 144.

is Sparks et al., 1964, p. 178
}

${ }^{36}$ Duncan. 1964, p. 144. 


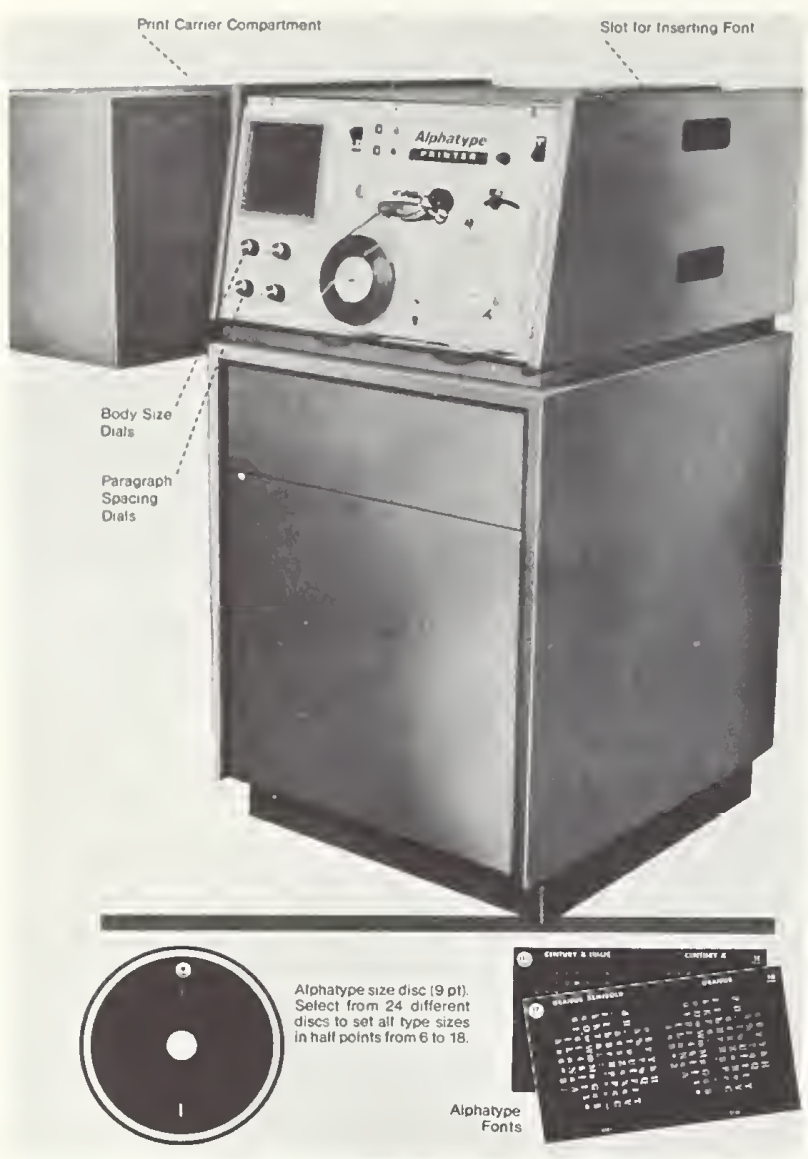

Figure 34. Alphatype photocomposer.

That is, "justification operations are calculated on the recorder and inserted when the carriage return-line-feed button is pressed." 37 This tape is read into the photo unit at a speed 30 percent faster than the recording speed. Maximum recording speed is about 8.5 characters per second, and the corresponding playback speed is about 11 characters per second.

Keyboarding speeds are reported as up to 60 words per minute, and typeset copy "is composed at speeds up to thirteen characters a second on the exposure unit in reverse order onto film or photopaper." 38

The optical characters are contained in a manually-changeable grid of 84 characters. The grid has two positions, automatically selected, and there are 3 shutters, 5 flash tubes, and 21 lenslets to provide selection and optical gathering.

A Japanese spinning disc photocomposer, the Sapton, is reported to have speeds of about 6-7 characters per second. ${ }^{39}$ A special case of a spinning disc photocomposing device is the PHOTODRAFT system of the Keuffel and Esser [K \& E] Company which allows an operator to transcribe

\footnotetext{
${ }^{37}$ Duncan, 1964, p. 147

an Ibid.

39 "Filmsetting and Cold Type Methods", 1964, v, 7
}

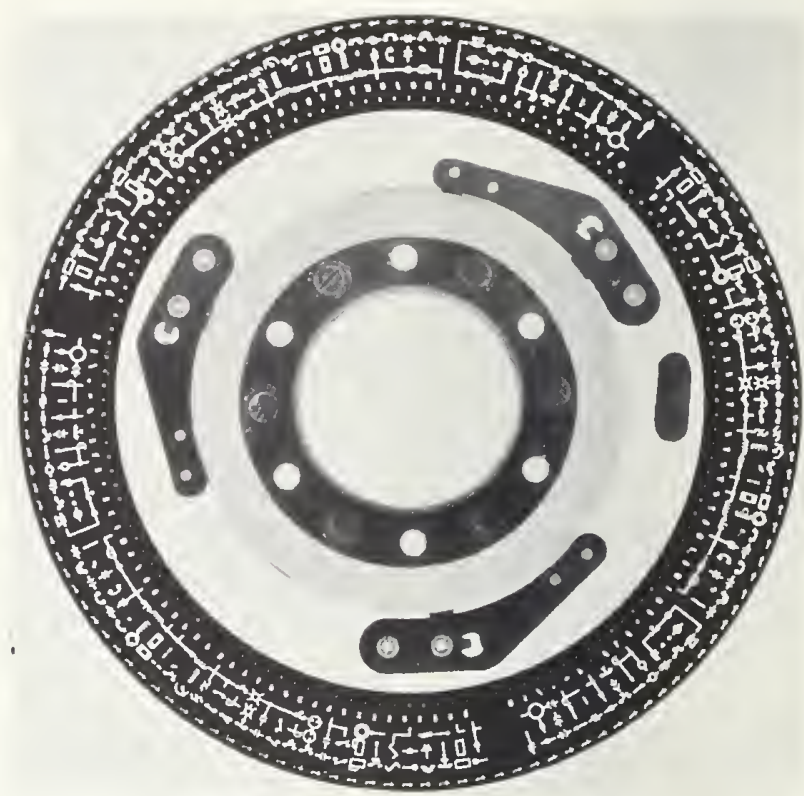

FI,URE 35. $K \&$ E engineering symbol disc.

from an engineer's sketch on graph paper lined for synchronization with the keyboard unit to produce tape to control the photocomposer and prepare a finished schematic. Up to 168 symbols are available, as shown in Figure 35.

Photon's development of the GRACE (Graphic $A$ rts Composing Equipment) for the National Library of Medicine provided the basis for the commercially available equivalent, the ZIP. The Photon "ZIP" or 900 series photocomposers, introduced in 1964, represent a substantial operational breakthrough in the speed barrier, permitting composition at speeds of 300 to 500 characters per second, fully justified lines up to 8 inches in length, with a choice of 264 different characters while operating on a single pass.

Operating principles of Zip are illustrated in Figures 36 and 37 . The only moving parts in ZIP are (1) a travelling lens that traverses the page horizontally composing a line with each sweep; and (2) the film advance mechanism. The mass of the lens is intentionally kept small. Stationary matrix plates contain the character images. By means of an optical device comprising two parallel mirrors, all the images in the same vertical column are directed to the same point of the film. Instead of a single light source, ZIP uses a separate flash tube for each character. The flash tubes flash at the precise time that the travelling lens is in the proper position with respect to the character matrix. The electronic timing system takes into account the proper escapement for each character, the value of word spaces, the location of each character on the matrix plate, and the position of the travelling lens. The film advances to the position for the next line during the lens return stroke. 


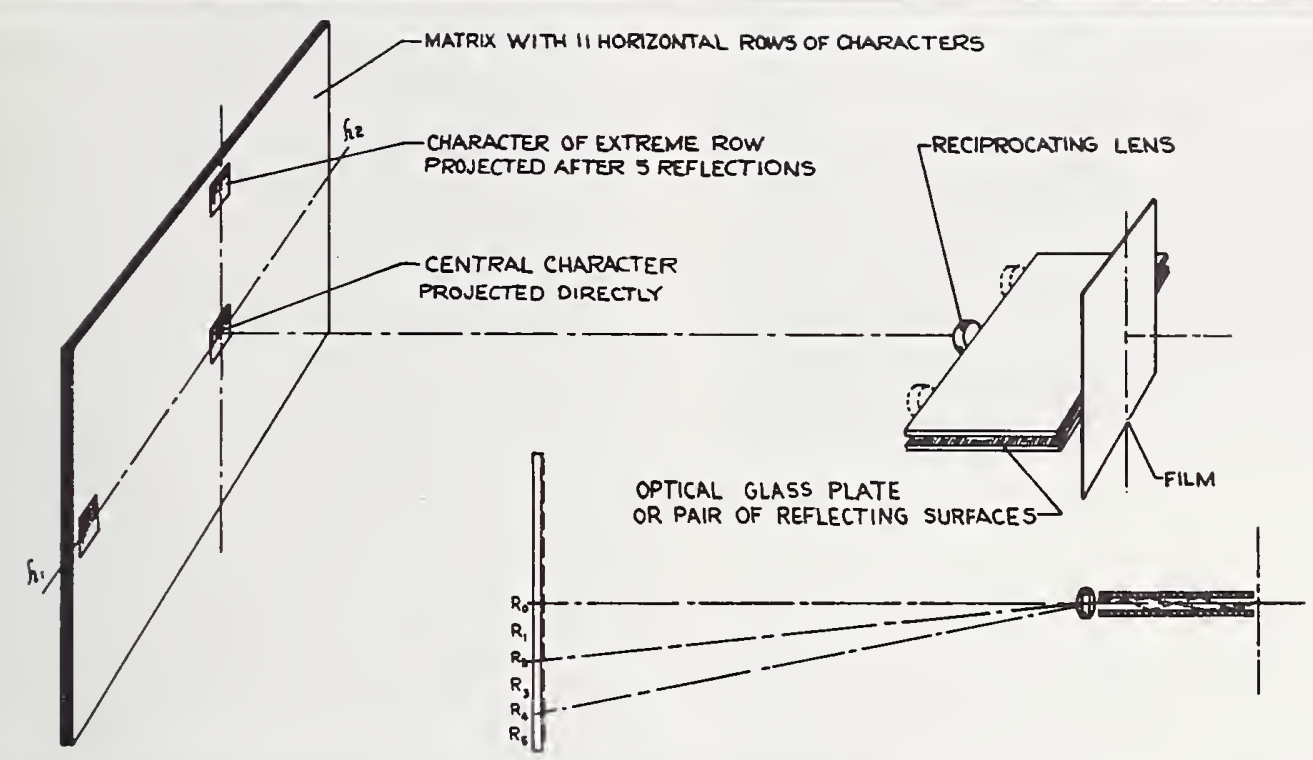

Figure 36. ZIP optical system.

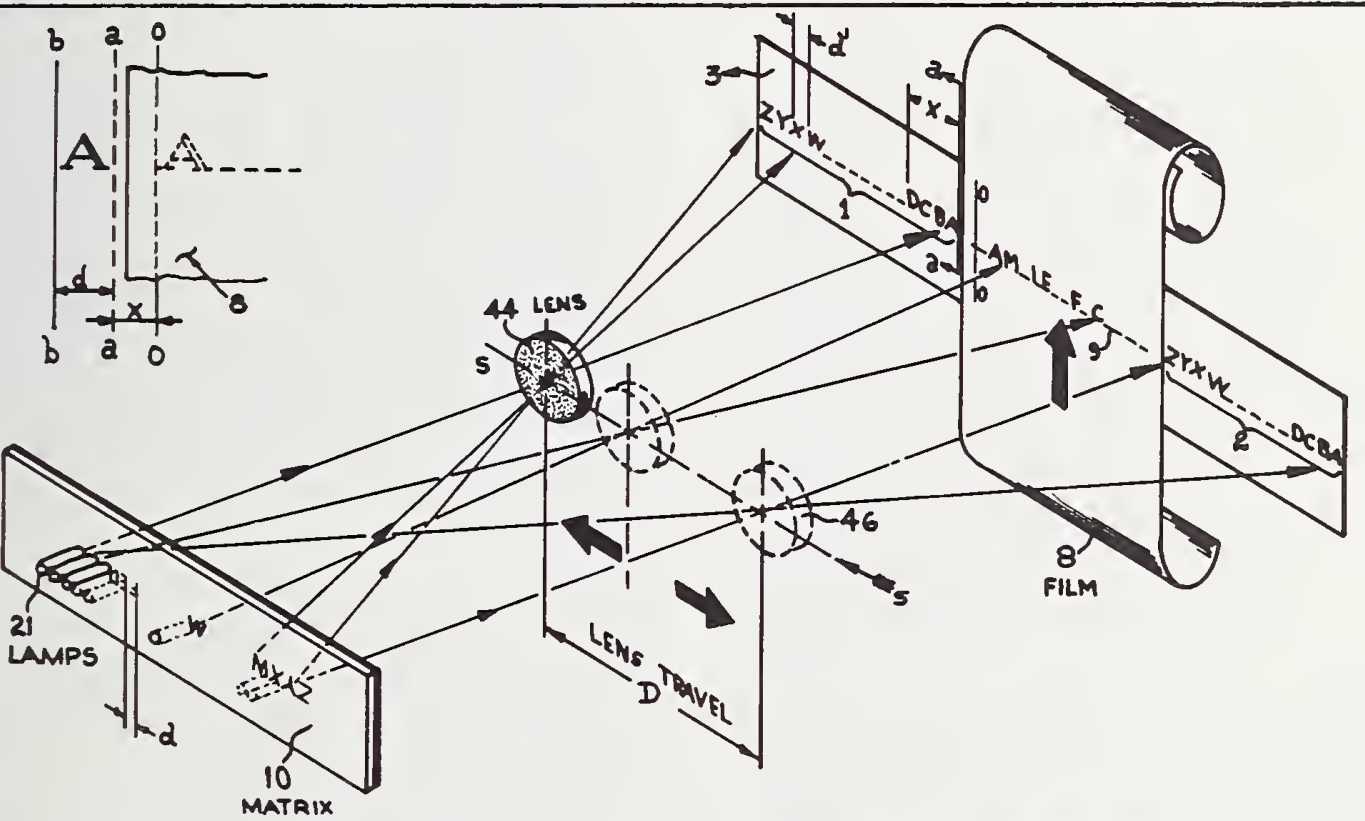

Figure 37. ZIP reciprocating lens.

The type matrixes are manually interchangeable, permitting a wide choice of fonts and symbols.

The first machine of the ZIP class, known as GRACE, was delivered in 1964 to the' National Library of Medicine, at Bethesda, Maryland, where it is currently composing the massive monthly Index Medicus. (Figure 38.)

On the day of installation at NLM (May 20, 1964) one of the co-inventors of the Photon machines said that the new equipment represented "the first radical departure from all conventional typesetring machines as it is 20 times faster than any existing machine on the market." 40 He also stressed another feature of interest to designers of information storage, selection and retrieval systems: "By turning ... knobs [of the control console], you can select a given page from the roll of input magnetic tape, and cause this particular page to be composed. You can extract a page or special article. The National Library of Medicine will

\footnotetext{
40 Moyroud, 1964, p. 106
} 


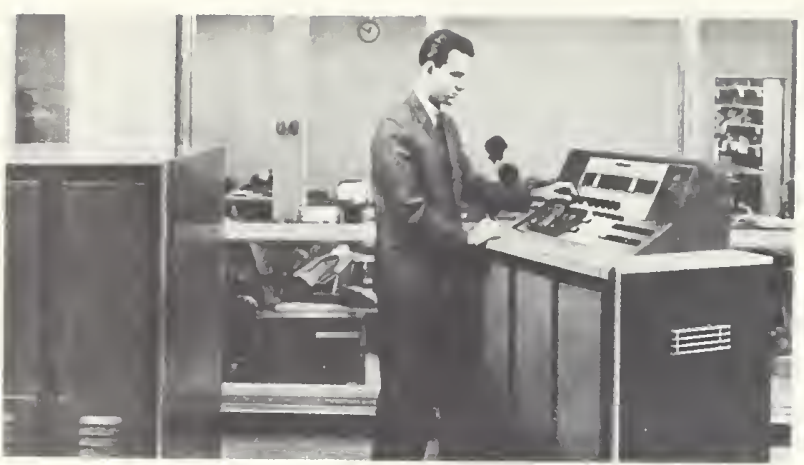

Figure 38. GRACE installation, National Library of Medicine.

utilize this feature to supply a print of an article ..." 41

The Mergenthaler Linotype Company has under development, in cooperation with CBS Laboratories, an all optical-electronic page composing photocomposer to be known as the Linotron. The first Linotrons are scheduled for delivery to the U.S. Government Printing Office and the U.S. Air Force in early 1967.

The Linotron has glass matrix grids of characters, similar to Linofilm grids except that there are 256 characters on a grid, and the grids may be selected and changed under automatic control. The entire grid is steadily illuminated and optically projected onto the light-sensitive cathode of an imagedissecting tube. This image dissector tube is similar in principle to the earliest television camera tubes. It produces scanned video electron beams from all 256 symbols, but only one of these beams is allowed to pass through an electrostatic wire grid selection matrix. This selected beam then passes through an electron multiplier, where it is amplified to become a video brightening signal for a high-resolution cathode ray display tube. The images of the symbols thus formed on this display tube are accurately controlled for size and position by electronic means, are optically imaged and photographed onto film. An entire page is composed while the film is held stationary.

Film advance between pages, and grid changes to accommodate additional fonts are thus the only mechanically moving parts in the Linotron system. A grid change takes about $1 / 2$ second. For this reason, the Linotron is programmed to compose all of the symbols from one grid onto the entire page. Then the grid is changed and the symbols from it are added to the page whenever required, etc. A grid change can occur during film advance to save time.

During composition of a page in the "galley proof mode" the Linotron can compose at a speed of about 10,000 characters per second. "If you can content yourself with lower output quality, the machine can do 10,000 characters per second at 6,8 , and 10

"Moyroud, 1964. p. 109
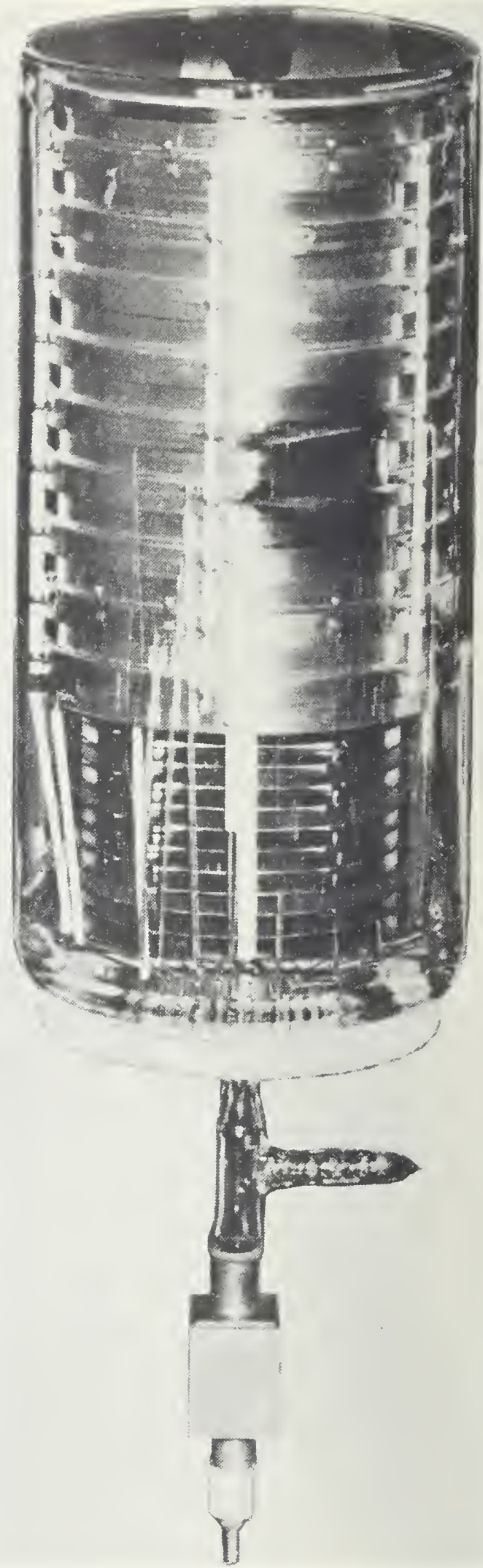

Figure 39. Linotron video device. 
points, or about 5,000 characters per second in all point sizes. What we do here is to cut out the alignment servo. The output is not perfectly aligned, but it is adequate for most proofing purposes." 42 When operating in its normal composing mode, with electronic servo systems operating to provide accurate placement of each symbol, then the speed of the Linotron is about 1,000 characters per second.

The Linotrons being built for the Air Force will also provide for the generation of graphical material, such as line art illustrations, continuous tone illustrations and screened halftone illustrations in a separate video display system, with optical mixing of the lexical and graphical material.

Because of its high speed, the Linotron will be driven by computer-generated magnetic tape, rather than by perforated tape, since the fastest available perforated tape optical readers do not read much above 1,000 characters per second.

As Duncan reported in 1964, "in addition to ZIP several other electronic composers are under development: One in England (with a specification similar to ZIP, but using a rather more highly developed keyboard for inpüt.

At the July 1964, Computer Typesetting Conference, Schwartz described the proposed Alphanumeric Photocomposer system to be operationally coupled with a computer sometime about mid-1965. While not intended to be made commercially available, the system as planned represents an interesting compromise between high speed $(4,000$ characters per second) and a less restricted character set $(270$ at any one time, comprising any 3 of up to 70090 character fonts available on magnetic tape) than most of the high speed character generators.

Some details of the Alphanumeric specifications are as follows: "The maximum line length the system can accommodate is eight inches of 48 pica. Printed type can vary in size from six to fourteen points. The only movable element in the entire system is the film transport which moves the film at constant speed past the character generator. When film is in the proper position for printing, an entire line of information is scanned across the cathode ray tube and is directed to the film via an appropriate lens. ...

"Type fount characters to be printed are previously coded and stored on magnetic tape which acts as a slow access bulk storage medium ... The computer itself can hold in rapid access storage over three founts of 90 characters each. Any of these 270 characters can be intermixed on a line ... When composing with six point characters, the output speed will be approximately 4,000 characters per second. It will be reduced to approximately 850 characters per second when printing fourteen point type." 44

${ }^{42}$ Corrado, 1964, p. 114

43 Duncan, 1964, p. 136

" Schwartz remarks, Computer Typesetting Conference "Proceedings", 1965, pp. 188-191.
Character patterns to be displayed via the CRT generator are encoded with a digitized coding scheme which minimizes the total information needed to be stored so that the equivalent of 63,000 different characters can be stored on a single reel of high density magnetic tape. It is further claimed that "the handling of characters in this manner allows the user the capability of designing, coding, storing, copying, and generating type fount characters of any design or size without dependence upon the manufacturer." 45

A slightly higher speed, and a more restricted character set is provided in the British Rank-Xerox Xeronic equipment, "which currently produces a 26 inch line of ten or eleven characters per inch at a rate of 2,880 lines per minute. The standard repertoire is fifty-six or 112 characters, and charac. ter generator designs have been produced for a number of relatively exotic languages. The output is produced by Xerography ... and pre-prepared material may be superimposed at the output from a store of thirty-two drawings held on a movable film band." 46

Several other phototypesetting machines in the speed range of several hundred characters per second are in various stages of design, prototype development and field installation and test. The CIS [Composition Information Services] Newsletter for August 1, 1965 reports on equipment demonstrated at an exhibition in Paris, including CAE's computerized typesetting system, a new tape merging device for Roboset, the prototype of a Monotype Computer Input Keyboard capable of handling 6-, 7-, or 8-channel tape, as well as the high speed CRT photocomposers of Dr. Rudolf Hell (Digiset) and the K. S. Paul (P \& M) Filmsetter.

"Using optical matrix CRT techniques, the K. S. Paul (P \& M) Filmsetter was designed primarily for text filmsetting at speeds from 150 to 400 lines per minute. This device is now ready for field-testing, and marketing should begin in England next year. A relatively low-cost keyboard, based on the P \& M linecasting tape keyboard, although somewhat more elaborate, has been designed for use with the Filmsetter". ${ }^{47}$

The Digiset developed by Dr. Rudolf Hell of Kiel utilizes an 8-bit character selection code and it sets justified and hyphenated lines of 11,18 , or 28 picas at rates up to 400 characters per second. Dr. Hell claims that higher speeds are possible:

"By means of indirect or direct control input over magnetic tapes the setting speed can be increased up to the limit of 2,000 characters per second. With modern electronic stores with 10 times higher working speed than the stores now in use, even higher setting speeds seem possible if film material and light emission of the picture tube would not ultimately set a limit." 48

\footnotetext{
${ }^{45}$ Ibid, p. 189.

${ }^{46}$ Duncan, 1964, p. 133

${ }^{47}$ CIS Newsletter, Aug. 1, 1965, p. 3

48 "Digiset", n.d., p. 4.
} 


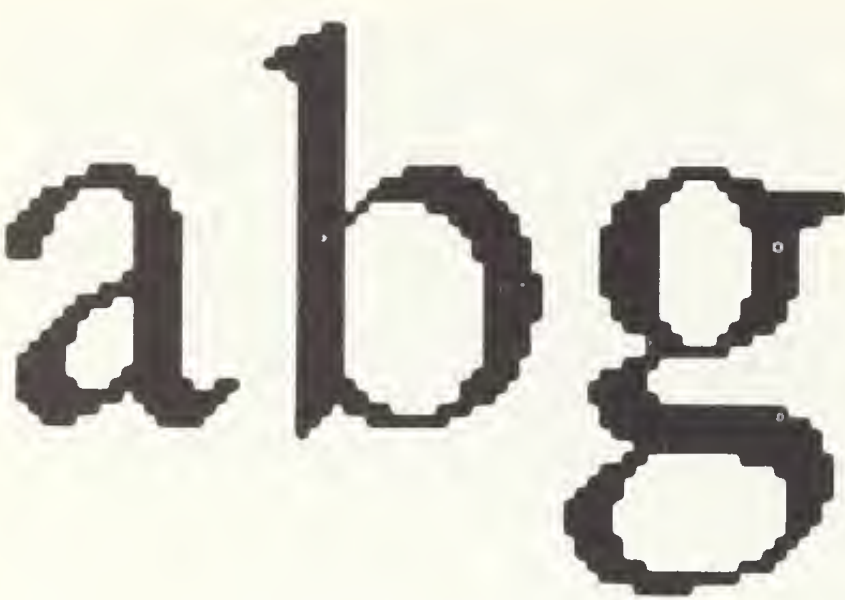

Figure 40. Digiset characters.

Character-dot resolution in the Digiset equipment ranges from $48 \times 10$ to $48 \times 46$ in 6 point type. Figure 40 illustrates enlarged characters as they are generated and viewed on a projection screen. In a telephone directory application, the character set consisted of 180 alphanumeric and other symbols. Character patterns are stored in a core memory of $180,000-190,000$ bit capacity. A character set may be replaced with an alternate set of 180 characters by transfer from auxiliary store in approximately one-half second.

\subsection{Electronic Character Generator Systems}

For the most part, electronic character generators developed to date have such limited repertoires of character sets that they do not as yet offer the typographic quality and versatility required in most scientific and technical documentation applications. An obvious exception is the Linotron development.

Their great virtue, of course, is their extremely high speed which may extend up to and even beyond 60,000-70,000 characters per second. Improvements in size of character repertoire and for rapid switching under automatic control are under active development. In addition, some systems incorporate interesting potentialities for program-controlled, light-pen inserted, or reproduced-from-storage interpolations of graphic material with text, ranging from formatting grids and lines to separate or to emphasize tabular or columnar matter to the incorporation of reproduced photographs with half-tone fidelity.

A number of these developments were foreseen by potential commercial suppliers, at least four or five years ago. In the Syracuse study for the Rome Air Development Center on "Lexical-Graphical Composing Techniques", Buck and his associates report, for example, that: "At the present time, the Recordak DACOM System displays up to 60 different alphabetic, special or numeric characters ... The 14-line raster scan employed in the present DACOM system permits a wide variety of type styles. However, only one font can be used at any given time. A photographic plate, containing 60 characters (the image source of the character generator employed by DACOM) is conveniently inserted into the side of the apparatus . . .

"[However] future DACOM apparatus is proposed to include the following features: 1) complete sets of fonts, as specified, to be displayed inter-changeably with true upper and lower case within each font, italics within each font as required, and a total number of characters ranging to 2000 or more; 2) proportional spacing; and 3) right hand justification." 49

The DACOM (Datascope Computer Output $M$ icrofilmer) equipment was apparently operated only for a year or two at the Washington Service Bureau of the Recordak Corporation, where it was shortly to be replaced by the General Dynamics Stromberg Carlson S-C 4020, despite earlier claims that, although "the unit first produced was geared to the associated IBM 727 tape drive, around 15,000 characters per second . . . with only slight modifications it could handle speeds up to 90,000 characters per second." 50

Before considering the S-C 4020, however, let us briefly outline the principal techniques used for electronic character generation to date, whether as yet used for photocomposition or computercomposed systems or not. Typically, the electronic character generators are computer driven and were developed to meet computer output requirements. Thus, the typical device operates upon the basis of digital codes generated by a computer.

\subsubsection{Techniques of Electronic Character Generation}

In outline, the principal techniques used for electronic character generation are as follows:

"Essentially, there are five distinct electronic and optical methods of forming or generating char. acters from digital codes.

1. The character can be formed by passing an electron beam through a stencil-like cut-out in the shape of a character located between an electron gun and the face of a cathode ray tube (CRT).

2. The monoscope method of character generation utilizes an electron beam that hits a metallized target within a CRT, with characters printed thereon, causing a video signal corresponding to the desired character, which signal is amplified and displayed on a separate CRT face.

3. A digitized matrix is generated wherein the character selected represents a series of intersection points with the raster lines of the matrix displayed on the face of a CRT.

4. A CRT scans a character mask, which is larger than the face of the scanning CRT and outside of its envelope, by using an optical tunnel, and

\footnotetext{
${ }^{49}$ Buck et al., 1961, p. V1-27-28.

so Webster, 1963, p. 27.
} 
the resulting video signal is displayed on the face of a separate CRT.

5. The characters are generated optically by flashing a light behind a rectangular matrix and directing it onto film by means of a pair of parallel mirrors, a traveling lens, and appropriate electronic timing circuitry." 51

\subsubsection{Specific Character Generator Equipment}

First and foremost among the character generator techniques embodied in equipment of direct use in composition-publication systems is the S-C 4020 (Figure 41).

The Stromberg-Carlson Division of General Dynamics Corporation has produced several dozen S-C 4020 systems during the past few years. These systems are photocomposers that operate from magnetic tape at speeds up to 17,400 characters per second. The repertoire of characters is obtained from a stencil matrix inside of a special cathode ray tube, known as a Charactron tube, which serves as the display tube. The repertoire is limited to 64 or 88 symbols, and those symbols which have isolated centers, such as "O" and "A", appear to be broken characters from the point of view of graphic arts quality, because of the visible tiny wires which hold in place the centers of these symbols in the beam-shaping stencil used to shape the electron beam into the shape of the symbol to be displayed. Serifs can be readily generated if they are included in the beam-shaping stencil.

Burger (1964) has described a means whereby a Charactron tube is used in the vector mode, in which the symbol "period" is selected continuously or intermittently, and deflected in accordance with a computer program to generate a variety of symbols.

Duncan comments: "As it is built at present the General Dynamics machine produces either $35 \mathrm{~mm}$ film or a paper copy (or a paper offset plate) and the character repertoire needs enlarging, but it is also capable of handling line diagrams, and adjustments of scale are possible either direct or from the film intermediate, which can be reproduced on a highspeed dry diazo microfilm enlarger . . ." 52

As an example of S-C 4020 graphic composition capabilities, the equipment has been used for generation of system and program flow chart diagrams, such as the automatic drawing of PERT charts, demonstrated by North American Aviation in 1963. ${ }^{53}$

Possible use of S-C 4020 and other General Dynamics equipment for the "lexical-graphical composer" (a proposed system to be capable of imposing illustrations and the like in proper page position with text or translated text) were noted by Buck et al., as follows: "The proposed Composer Printer is an adaptation of General Dynamics/Electronies S-C 4020 High Speed Microfilm Recorder with an F-50 (Tape Adaptor) option. This unit is

\footnotetext{
5. Sparks et al., 1964, p. 178.

s2 Duncan. 1964, p. 134

satamation 9. no. 6.80 (June 1963).
}

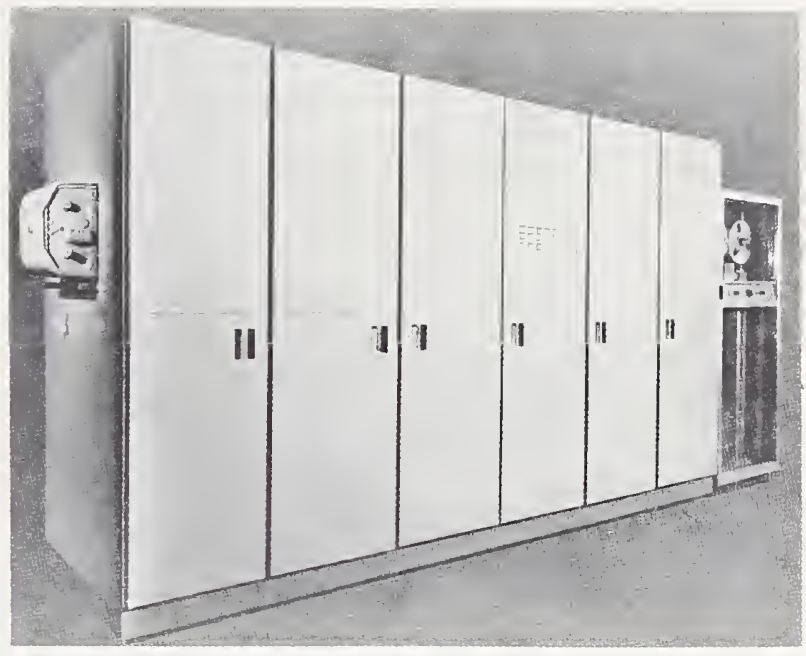

FiguRE 41. SC-4020 equipment.

specifically designed to print out alphanumerics from digital computer code. It is possible to project information previously recorded on film (graphics in this case) superimposing same on the alphanumeric display. The final output of the system is a $35 \mathrm{~mm}$ film." 54

"The S-C 3000 High-Speed Communication Printer . . . is designed to accept binary coded alphanumeric data from remote sources over telephone, telegraph, radio or other communication systems. It prints at rates up to 10,000 words per minute. " 55

"The S-C 5000 High-Speed Electronic Printer . . . (utilizes) the CHARACTRON Shaped Beam Tube in conjunction with the Xerox printer . . 100,000 words (5.000 lines) per minute. ${ }^{\circ 56}$

"The S-C 6000 Facsimile Printer . . receives incoming video signals, created by the [S-C 6400 Facsimile] scanner or other equipment and transmitted to the printer, which reproduces the original material on the face of a cathode ray tube. Print out onto hard copy is achieved by the Xerox process . . 16 pages $\left(8^{1 / 2} \times 11\right)$ per minute. Resolution is equivalent to 100 TV lines per inch." 57

Early disadvantages of the Charactron tube approaches led, for example, to developments at RCA involving fiber optics or optical tunnel techniques.

"The Charaçtron tube has a limitation in the matrix which contains in the order of 42 characters, and every time you change a font, you just den't change that matrix, you have to change the whole big Charactron tube." 58

Thus it was desired to provide a design in which different fonts or character sets could be interchanged and in which resolution would be of relatively high quality. "The fiber optics tube is

\footnotetext{
54 Buck et al., 1961, p. VI-29

ss lbid. p. VI -60

56 Ibid., p. V1-60, Vl-59

${ }^{57}$ Buck et al. 1961, p. VI-61

${ }^{5 *}$ Flint, 1963. p. 126.
} 
designed with 1,000 fibers to the inch in each direction. We don't actually get a resolution of 1,000 lines per inch, but we can come pretty close ... and this resolution is beginning to approach the kind of quality that a printer requires for his work." 59

Moreover, "the fiber optics tube . . can make use of an external matrix which can be inserted at will, so that you can change type faces, and have control over your end product, as you do in a line casting machine . . ."60

As of a year or two ago, it could be stated:

"The RCA developments have been described by Haynes who gives the design speed of 500 characters per second or fifteen lines a second, with a resolution of 400 lines to the inch (sixteen lines/ $\mathrm{mm}$.), and a repertoire of 275 up to 500 characters 'on-line.' It makes use of the 'optical tunnel' to improve the resolution of the system." 61

Developments at CBS Laboratories, particularly the cooperation with Mergenthaler from 1962 onward, have been noted in the discussion on the Linotron system. A related approach has been described as follows: "The CBS Vidiac 389-10 generator with a standard fount of sixty-four characters and the rather low resolution of ten lines per $1 / 8$ in. character will work at a speed of 50,000 characters a second. The modifications proposed for Graphic Arts quality will aim at a resolution of twenty line pairs per $\mathrm{mm}$. and speeds between 1100 characters a second for 6 pt. and 200 characters a second for 18 pt., with positional accuracies to 0.001-in. Basic character repertoire will be 256 chàracters, with alternative blocks of this size readily available for simple switching in and out." 62

Another example is the Videograph Printer which is produced by the A. B. Dick Co. This is a "high speed electronic page printer which is available in two types. One produces hard copy print out of fully edited six bit digital information at speeds of up to 20,000 characters per second. The other type reproduces facsimile copies of graphic material from video signals at rates of up to three $81 / 2^{\prime \prime} \times 11^{\prime \prime}$ pages per second. An electrostatic printing tube is used in both types of printers." 63

"The Alphanumeric Printer consists mainly of a character generator, an electrostatic printing tube, image-fixing device, and the paper transport mechanism. The character generator, which provides video signals to the electrostatic printing tube is essentially a cathode-ray tube having a matrix of small diameter wires fused in and through its face plate. The CRT electron beam, whose strength varies according to the video signal voltage received from the character generator, sweeps over the inner ends of the tube face wires. Electron charges are deposited through the wires onto the dielectric coated paper thus forming latent electrostatic images. . . ."64

\footnotetext{
5* Flint. 1963. p. 126.

(6) Ibid., p. 126.

61 Duncan, 1964, p. 136

${ }^{62}$ Ibid.. p. 136.

${ }^{3}$ Buck et al, 1961, pages VI-5I.

Hat. Ibid. pp. V1-51.
}

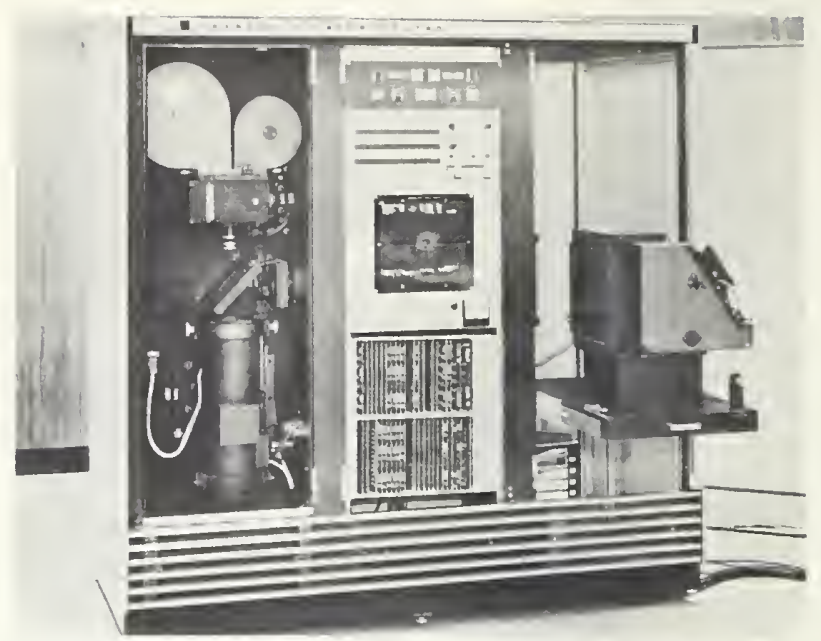

Figure 42. Benson-Lehner Transdata equipment.

"The Electrada Typoscope is a compact, nonflickering display system ... currently capable of performing the display operation at rates of up to 15,000 characters per second.

"Expensive and complex electronic storage and character forming systems are avoided by utilizing a unique shaped beam, direct-view storage tube. Upon receipt of the code for a particular character, the electron beam is passed through a stenciled matrix, deflected to the desired screen location, and stored within the tube by means of a suitable dielectric mesh. A total of sixty-four characters are available in this matrix. . . ."65

"A Simpix electron gun can also be incorporated in the Printapix system ... designed to provide extreme ease of character selection by accepting six channel binary data from a computer and automatically selecting the proper alphanumeric characters. This can be done at rates of up to one million characters per second. The size of the character printed can be varied by means of one voltage thus permitting the generation of subscripts, capitals, and italics (by a slight rotation of the electron beam), etc., utilizing a single character matrix. Extremely high resolution of up to some 150 to 250 printing lines per inch is possible. . . By the use of several tubes or several passes of the paper over one tube, color can also be incorporated in this print out system." 66

The Benson-Lehner Transdata (Figure 42) is also included here because of its claimed high speed of 62,500 characters per second and its optional line drawing and format superimposition features. The usable character set, however, is limited to 63 characters at one time, each defined by a $15 \times 16 \mathrm{dot}$ matrix in CRT character generation. Three variations of character sizes and four different character orientations may be program-selected. The 944 model is claimed to be compatible with various magnetic tape transports and to accept binary-

\footnotetext{
65 Buck et al., 1961, p. VI-39.

${ }_{66}$ Ibid., pp. VI-58,
} 
coded-decimal data. An earlier model, the 943, accepted binary coded data only. Output is to either $35 \mathrm{~mm}$ film or hard copy.

A special advantage offered by this equipment for book composition is cited by Duncan as follows: "The task of producing scrambled and upside down composition that may be required for book production, and may even be necessary for some news. paper operation, seems already to have been solved satisfactorily on one computer output printer-the Transdata. The processed pages can be stored on magnetic tape in any order required, or in random order, and called out in any order desired." 67

\subsection{Computers Used for Typography}

From the slowest photocomposers operating at less than ten characters per second through the intermediate range of several hundred cps to the fastest electronic generation systems it is to be noted that, in general, size of character set must be traded off against speed. In almost all cases, however, other aspects of at least partial automation of the compositor functions can be achieved by built-in computer-like calculations in special keyboards, by the use of small special purpose computers and by programs in large general purpose computers producing as output both text and control codes to operate printing equipment.

\subsubsection{Small Special-Purpose Typography Computers}

If some of the compositor functions are performed in an intermediate computer, there are usually two tapes involved. Both tapes may be perforated or magnetic. or one may be perforated and one magnetic. A code conversion is not always necessary, but if a reasonably complex computer is available as a composition aid, then code conversion generally does not pose a great penalty. In some systems employing a general-purpose computer, the input tape is the one most conveniently generated by the perforation keyboard. Its code may be converted by the computer to the internal code of the computer. After typographic processing, this code may again be converted by the computer to the code and tape employed by the typesetting machine. In computers designed for typography processing, these conversions can be avoided.

The simplest class of special-purpose computers perform the repetitious and arduous tasks of line justification, including variable spacing control and automatic insertion of line formatting controls, as well as automatic or semi-automatic hyphenation control. Computers which accept unjustified tapes and generate justified, hyphenated and formatted tape include the following:

a. Compugraphic Directory Tape Processor

b. Mergenthaler-Compugraphic Linasec

c. Fairchild Graphic-Thompson Ramo Wooldridge Comp/Set Computer

\section{d. Harris-Intertype-Cognitronics Editape Com- puters \\ e. RCA 30 Newscom Computer}

These computers permit faster keyboarding of tape by less skillful operators using simpler machines which are not encumbered by justification counters. Format controls, such as carriage return, inserted by the keyboard operator are either omitted in the tape or ignored by the computer, which inserts new format controls for the justifiedhyphenated output tape. These computers can be readily modified for font, point size and format changes. In the Directory Tape Processor, Linasec and small Editape machines, words requiring hyphenation are displayed to an operator, along with the allowable hyphenation range that will permit justification. The operator must then decide where the hyphen is to go, and press a button corresponding to that position.

In more complex computers, hyphenation is achieved automatically by table look-up or by the application of logical rules to the letter structure of the word. Neither type of system is completely reliable because of ambiguities, dual meanings, and different hyphenations for identically spelled words, and other quirks of natural languages. Some systems use a combination of logical rules plus table look-up for anticipated exceptions.

Use of the less complex devices in practical operation is exemplified by the Linasec installation at Alden Press and the use of Compugraphic Directory Tape Processor for telephone directory listings.

At Alden, the system involves 4 tape punches and 4 tape-operated linecasting machines to produce output of an estimated 2,560 lines per man-day by comparison with 1,472 such lines in the manual system even with the relatively slow (45 characters per second) first model Linasec reader. A console is provided which continuously indicates to the operator the point on hard copy corresponding to the punch position on the output tape.

Morton describes some further details of operation as follows: "If 65 percent of the available space band extension will not expand the line . . . signal lights on the console, over the characters on the hard copy, will indicate the letters following the filled line. The operator will count back the number of spaces from the last line to a proper point for hyphenation, and he will punch a button numbered on the corresponding responses to the number of responses so counted. The computer will then insert a hyphen and an elevator code and continue to operate line after line until the next operator decision is called for.... A change in font is handled by removing the 3 -by-5-inch plug . . . and replacing it with one designed for the particular font to be used. This operation of taking out a plug and putting another in place takes from 5 to 10 seconds." 68

\footnotetext{
Mforton, 1963, pp. 98-100.
} 
Swanson reports use of the Directory Tape Processor for telephone directory listings in part as follows: "After the name and address of a line have been set, one special key stroke signals the Processor to do at least three things:

a. Calculate and insert the leaders required.

b. ln a telephone number composed of letters and digits, make the letters in caps and the digits in bold face.

c. If there are no letters in the telephone number, set the seven digits in bold face." 69

Among the advantages and operational gains claimed are the following: "Our teletypesetter production of 15 pica lines has increased from 100 to 125 lines per hour to 250 to 300 . Eight-hour averages of 310 to 315 lines per hour have been made by some operators using Friden Flexowriters. . . . By using the Directory Tape Processor we have reduced our operators' and linecasting machine errors from 10 percent to 4 percent of all lines cast. . . . In an average telephone listing line of 40 characters, including leaders, we save approximately 20 key strokes. The strokes eliminated are the control and functional ones that are usually difficult for operators to master and, therefore, take more time than the regular alphabetic or numeric keys." 70

Compugraphic also produces the Tabular Tape Processor which uses plugboard wiring to set column widths for multi-column tables. "Replacing the time-consuming, arithmetical process of figuring the "cast' of a table, the TTP requires ... coded instructions on the tapes to tell the TTP where to fill a column with blank space (without regard to how many spaces are required), where to fill with leaders, when the end of a column has been reached. and the end of a line." 71

A recent development for directory composition outside the United States and the United Kingdom is to be noted as follows: "It is reported from Milan that SEAT are just about to change over to magnetic tape storage of Italian telephone directories with computer controlled updating and output ready for composing machines." 72

The RCA 30 Newscom is essentially a cut-down version of the company's 301 computer, having a $20 \mathrm{k}$ core memory, and capable of carrying out line justification and automatic hyphenation following the Los Angeles Times program. When fitted with a 100 character per second reader and punch it produces some 5,400 column lines per hour with higher speed options up to 17,400 lines per hour also available. ${ }^{73}$ Typical Newscom installations are diagrammed in Figure 43, and equipment is shown in Figure 44.

\footnotetext{
es Swanson, 1963, pp. 93-94.

${ }^{70}$ lbid, pp. 92-94.

Yasaki, 1963, p. 31.

Duncan, 1964, p. 149.

Duncan. 1964, p. 146; Dalamalion, 9, No. 12, 67 (1963); Dala Processing, 6, 38, 39 (1964).
}

With respect to the TRW (Thompson RamoWooldridge)-Fairchild equipment (Figure 45) it is reported that it will "run 9,000 newspaper lines per hour with standard equipment. With special equipment this can go up to as high as 27,000 newspaper lines per hour." 74

\subsubsection{General Purpose Computers Used for Typography}

In a special sense, the large, high speed, general purpose computer plays several different roles in modern developments in automatic or semi-automatic typographic quality composition.

In particular, "There are two entirely different uses of the computer, one is to produce something which has gone through the computer because the computer is serving a function in compiling the output and has been an essential part of the mecha. nism. The other is to use the computer to save some of the operations which are now done by the skilled keyboard operator."75

Certainly, the advent of the electronic computer has focused new attention on the problems of automated typographic composition. Automatic data processing has not only made it possible to organize, assemble, and produce many existing types of publications quickly, automatically, and sometimes at lower cost, but it has also created a proliferation of new types of reports, indexes, and publications. The basic content of many new types of reports including for example, key-word-in-context (KWIC) indexes, mathematical tables, accounting and financial reports, and U.S. Census Bureau reports, is produced by the computer.

To date, the natural tendency has been to utilize the high speed computer printout as camera-ready copy without regard to the readability of the output or to the size of the printing run. This creates certain disadvantages including poor typographic quality and higher printing costs resulting from the lower type density of computer printout, and inefficient utilization of white space unless carefully planned.

On the other hand, a manually composed, high quality publication using the computer printout for working copy defeats one advantage of the computer system-its rapid response time. For this reason, several companies are developing high speed composing machines that will operate directly from the perforated tape or magnetic tape produced by a computer.

We have already witnessed the wide usage of mechanical computer output printers for publication of stock market tables, census reports, bookform indexes, and the like. During the next decade, we will undoubtedly witness an expansion of these applications to include machine translation, automatic indexing and abstracting, dictionary com-

\footnotetext{
${ }^{74}$ F. S. Angell, remarks, Computer Typesetting Conference "Proceedings", 1965.

p. 180. p. 78.
} 

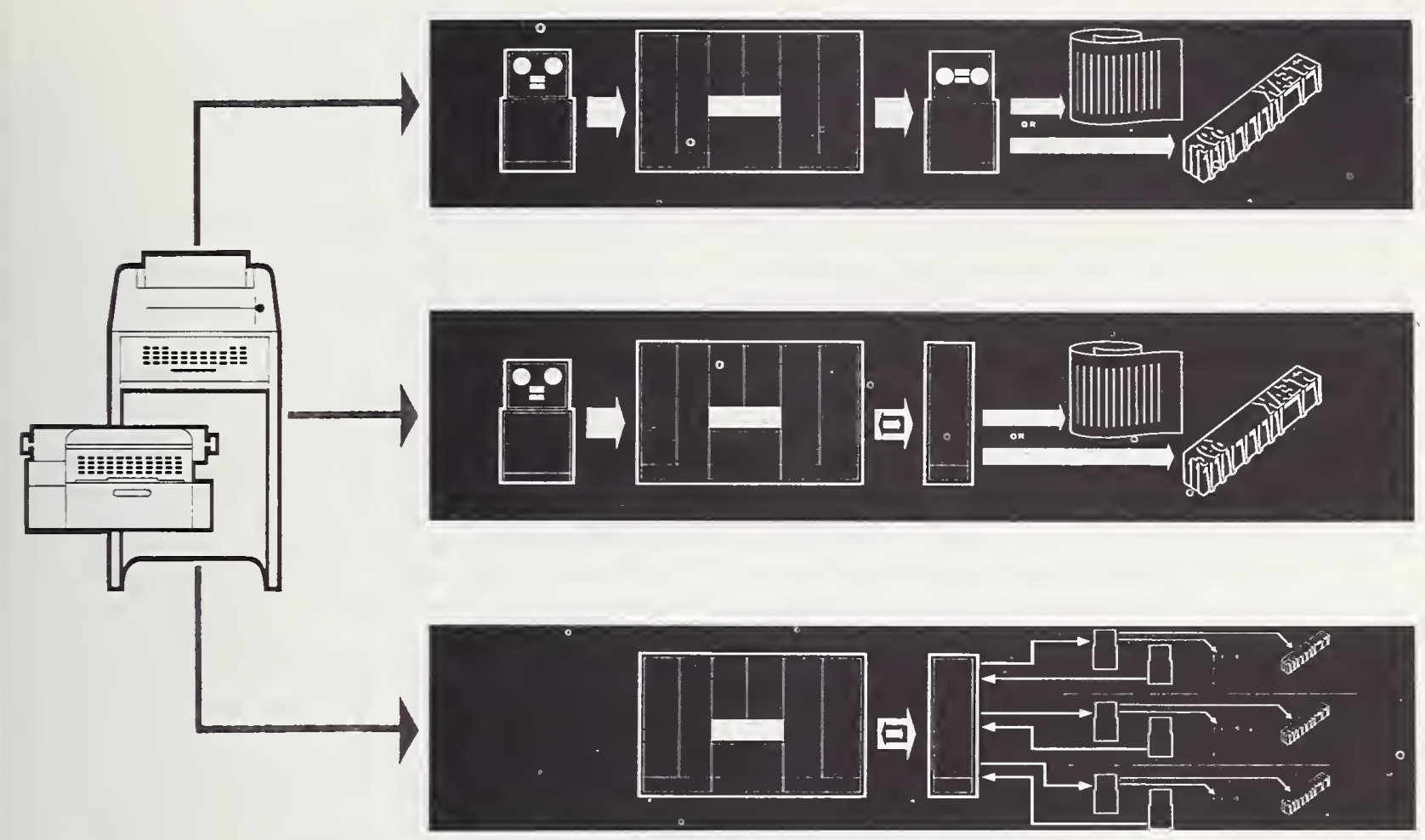

Figure 43. RCA Vewscom system.

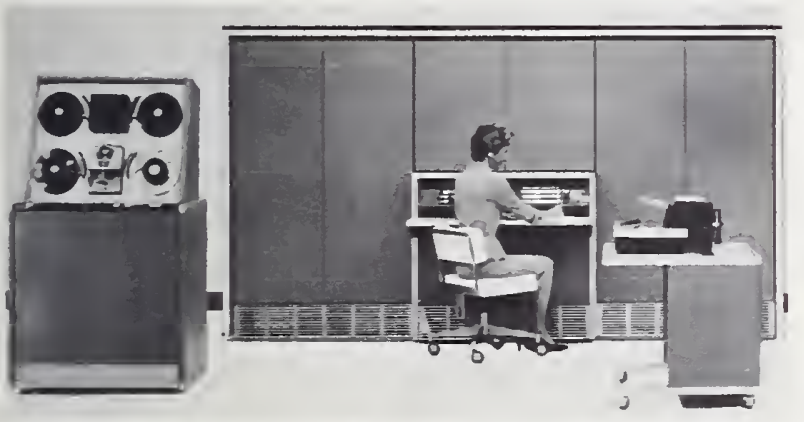

Figure 44. Neuscom equipment.

pilation. display advertising. scientific textbook composition, and many more, through the medium of high speed, graphic arts quality composing machines operable from computer readable codes.

Typical adverse comments on the practice of using high-speed computer printout are as follows:

-A major disadvantage of computer print-out is its poor legibility and its low information density. . . . The U.S. Air Force prints something like a billion and a half pages from computer print-out just in the one area of its stock list catalogues. . . We have found that we can pack three times as much infor. mation a page if we use Linotron typography, which

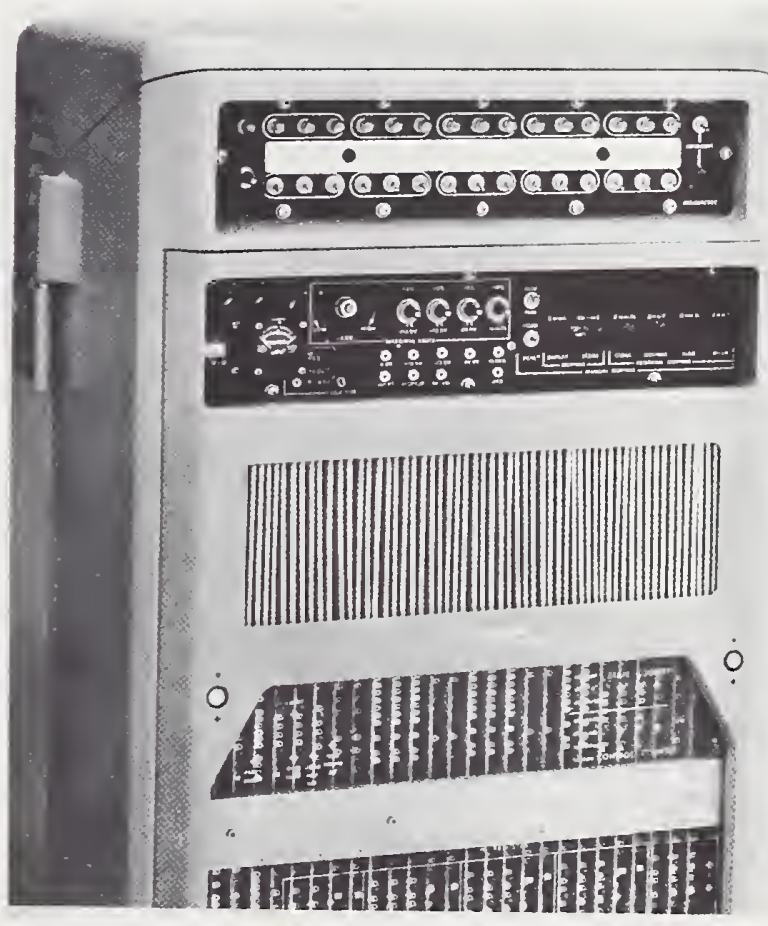

Figure 45. TRW-Fairchild Comp/Set. 
gives quite a saving-more than the cost of the machine" $" 76$

The principal benefits to be derived from employing electronic data processing equipment in a typographic composition system are a reduction in manual clerical effort, a reduction in response time, and an opportunity to use each record for inultiple purposes. The reduction in manual clerical effort can be realized through reducing the amount of keyboarding required for input. Textual matter that is to appear more than once in any publication or that is to appear in more than one publication need be keyboarded only once. The keyboard operator need not be concerned with justification. The data processing equipment can duplicate the record as many times as is required. Response time is reduced by the speed with which electronic equipment can retrieve information from tape, sort it into publication sequences, and produce an edited output. Thus the cut-off time for entering new information into the master record file need not be more than a few hours prior to the time that the edited tape is required.

With the file of textual information stored on magnetic tape, the data processing system can use the same information over again for different publications, for new editions of a previously published document, or as a source of information for responding to literature search requests. Thus, once a record is stored in the file, it may be used for many purposes without requiring further manual operations.

In addition to its normal function in the typographic composition system, the master record file can be 11 sed as a source of information for answering specific inquiries. This adds a literature search capability wherein the allowed complexity of the inquiries and the content of the answers depend on the extent and organization of the master record file. Responses to search requests can be in the form of lists of references to pertinent documents or in the form of references and abstracts, if the texts of abstracts are included in the master record file. In extreme cases, the entire text of selected items may be printed out.

Assuming that keyboarded tapes can be read into a general purpose digital computer, then the subsequent processing for typography (or any other purpose) is a function of the ingenuity of the programmer, the investment in his time, and the limitations in memory size, processing speed, and output capability of the computer.

At the U.S. Government Printing Office, a system has been evolved using Friden LCC (Line Casting Control) Justowriters equipped to punch Teletypesetter code in a l-inch paper tape of the usual variety, with sprocket hole centers in line with code hole centers, rather than advanced-feed sprocket holes as in conventional TTS tape.
These paper tapes are read into an IBM 1401 computer. After processing, the computer produces a 1/2-inch magnetic tape with the composed data. The data is in the format of the Mergenthaler Linofilm photocomposer, except that the data for one Linofilm character (15 bits) is distributed over three consecutive magnetic tape characters, each containing up to 6 information bits. These magnetic tapes are then read into a magnetic-tape-to-Linofilm-perforated-tape converter, which can punch 300 Linofilm characters per second by means of a specially adapted Soroban high speed tape perforator. These Linofilm tapes are then ready to drive the Linofilm photo unit, at its characteristic speed of about 12 characters per second.

It has been the experience at the Government Printing Office, using the above system, that about half of the programming effort and half of the computer time is devoted to control of the format of the printed page. Many formats are now available for production use. A system has been devised for detailed control of the line-by-line formats, including indentations, font changes, point size changes, quadding, carry-over of headings, and the like, using a "punched card image" of the desired format. There is a straight forward correspondence between hole positions punched in the image card and indentations and line spacings for various categories of the printed material.

The list of general purpose digital computers used for some phases of typographic composition is growing steadily. The following is a list of American-made general-purpose computers known to have been programmed and used for typography:

$\begin{array}{ll}\text { RCA 301 } & \text { IBM 7094 } \\ \text { IBM 1620 } & \text { IBM } 360 \\ \text { IBM 1401 } & \text { Honeywell } 800 \\ \text { IBM 701 } & \text { NCR 315 } \\ \text { IBM 704 } & \text { GE 225 } \\ \text { IBM 709 } & \text { Control Data } 8080 \\ \text { IBM 7090 } & \end{array}$

Examples of computers used elsewhere than in the United States for similar purposes include the British Ferranti computers Argus 104. Pegasus, and Orion, the English Electric KDF 9 installed at the University of Newcastle upon Tyne in mid-1964, and also in Great Britain, the Elliott 803 B. As another example, it is reported that, ". . . The Compagnie Europeen d'Automatisme Electronique $(C A E)$. . is now ready to supply a computer typesetting equipment, based on the CAE 510 computer." 77

A special case involves the use of the SDS-910 computer as programmed controller of an automatic character recognition device, the Electronic Retina Character Reader of Recognition Equipment Inc., to accomplish justification, hyphenation and editing for both automatic typesetting from

${ }^{77}$ G. Bafour remarks, "Computer Typesetting Conference Proceedings". 1965. p. 195 
typewritten copy and the input of original text to machine translation systems. ${ }^{78}$

Examples of development of general purpose computer programs and applications to problems of documentation will be discussed in a later section of this report. Perhaps unusual in any area of emerging technologies is the fact that, in general, "the cobbler's children" have been shod. Bozman. Barnett, and Duncan provide well-known examples. The report on Graphic Composing Techniques is another. More recent illustrations are also to be noted:

"All the text has been typeset by computer methods: part of chapter 4 by the Rocappi general purpose computer system, and the rest by the Linasec special purpose computer method." 79

"A data sample of two journal article manuscripts, 20 first pages of journal articles. and journal volume index (for one year) of titles and authors have been processed." 80

"All of the copy in this appendix was typeset automatically, on a tape driven Model 200 Photon, from original journal machine record with the following exceptions.

(1) Figures and multiple line equations were exposed from the original copy and stripped in.

(2) The index machine records were sorted manually, because sort programs were not available at the time the data was processed." 81

IBM has issued a program called Text-90 to accomplish computerized typography, using punched card input of mixed text and controls, for processing on a 7090 computer, including justification, hyphenation and formatting into one or two columns per page. Three magnetic tapes are produced, called a print tape, an exception tape and a master tape. The exception tape is used by the program to produce automatically tables of contents, indexes and lists of misspelled words. After listing, editing and correction, the exception tape is reprocessed by the program to produce the final print tape, which is fed into an IBMI 1401 computer where a special utility program lists the tape on a 1403 chain printer. If a special 120 character chain is employed, then the document is printed in both upper and lower case. If only a standard chain is available, then the document is printed in all upper case. The master tape contains text and controls, and is retained for use in producing revisions (updating) of the document.

The remainder of this report will be devoted to a review of known attempts to automate various portions of the compositor functions, and to some the documentation applications, problems and prospects that can be foreseen. The material to be covered will be arranged somewhat in order of increasing complexity, somewhat in order of chronology, and somewhat in order of function, but it

\footnotetext{
Le Brun, 1963, p. 150; Data Processing Magazine 7, 47 (February 1965).

Ts Allen. 1965. p. viii.

Buckland. 1965. p. 2

Ibid., Foreword to appendix.
}

will necessarily remain somewhat disorganized, since the various techniques, systems and available hardware do not fall clearly into distinctly separable categories. Rather, they overlap in varying degrees as was evident in the present section describing typesetting equipment.

Turning now to semi-automatic and automatic techniques and devices to accomplish many of the compositor functions, we will consider the questions of horizontal line justification and hyphenation, vertical justification and page make-up; the insertion of corrections, editing and merging; the typesetting of two-dimensional graphic material, and the intermixing of typeset text with facsimile replicas of graphic material such as drawings and photographs.

\section{Automation of the Compositor Functions}

Perforated tape itself is an intermediate automation aid, since it can, when fed into a tape reader connected to an "operating unit," operate a typesetting machine, hot metal or phototypesetter, at a consistent speed limited by the typesetting machine rather than by the speed of keyboarding. Thus, maximum utilization is obtained from the composing machine, which may absorb the output from several keyboard tape perforators.

If the perforated tape is the only automation aid employed, then most or all of the compositor functions must be concentrated in the keyboard operator. In such case, it is essential that the same tape and tape code required by the operating unit be generated by the keyboard perforator. Otherwise a code conversion and generation of a new tape is required, which is unnecessarily costly and time consuming, nullifying some of the advantages of perforated tape. Thus, where perforated tape is the only automation aid employed, the tape and code used by the operating unit should be generated by the keyboard perforator.

Magnetic tape systems are being developed employing "stepping motors" to give an incremental advance for each character. These incremental tape systems can perform all of the functions performed by perforated tape, but they do not offer the same ease of visual editing and correction. They can, however, be read into and out of a computer at much higher rates than perforated tapes. Perforators can punch 300 characters per second, and the fastest optical tape readers read less than 2000 characters per second, whereas magnetic tapes are typically "read" and "written" at rates of 10,000 to 100,000 or more characters per second.

Since the principal methods for achieving a desired type density obviously relate to the prohlems of close and even spacing, both horizontally with respect to the line and vertically with respect to the column or page, we shall consider here the problems of horizontal and vertical justification 
and the concomittant difficulties of word-hyphenation or the avoidance of "widows," together with both the difficulties and advantages promised by semi-automatic and automatic aids to the accomplishment of these compositor functions.

\subsection{Horizontal Justification and Hyphenation}

For high typographic quality publications, the typographic composition system must perform both horizontal and vertical justifications. If these functions are to be performed automatically, the equipment used in the system must be able to determine what combination of line structures will minimize the number of hyphenated words, to adjust the spacing between characters so that margins are aligned, to adjust spacing between lines so that subheads are not placed at the bottom of a column, to change margins, to indent lines, and to center lines. Each of these operations can be performed by available data processing systems if the appropriate specifications are included in the computer program, or in the input records, or can be entered via punched cards or punched tape as an input to the cycle.

Barnett, Kelley, and Bailey have summarized the operations required to effect horizontal justification in these terms:

"Justification can be regarded as the expansion of a line of type to some specified length by an increase in the spaces between appropriate pairs of adjacent characters. In describing a justification procedure, it is necessary to specify (i) the minimum space to be left between different pairs of characters, (ii) the spaces which may be expanded, and (iii) the limits that will be placed on the allowed expansion. In general, justification will be effected by increasing the distance between successive words for which some minimum non-zero spacing has been specified. In the simplest case, each such space along a line is increased by the same amount. At times it is necessary to stipulate that only certain of the spaces in a line of type may be expanded. A further method of justification that will be used quite often involves the expansion of non-zero spaces to some preset limit, with the distribution of any further spacing that may be necessary between characters whose fields otherwise would touch." 1

It is to be noted, however. that ". . . Some fixed limit must be placed on the justification range otherwise the word spaces in the justified line might become too large. This would result in a waste of page space and in unacceptable typography that is characterized by "rivers' of space which pervade the text column and make it relatively difficult for the eye to read.".?
In manual control of hot metal casting equipment, Lorizontal line justification is typically accomplished as follows:

"By restablishing the cut-off point fof the line]. the ajerator is satisfied that the aliable width expansion of the space bands, which are arljustalole slid ing wedges, is sufficient to expand the width of the line to the width of the mold at which the line is cast. Thereafter the assembled line containing the matrices and space bands is positioned at the mold face and the space bands in the line are expanded by ram action ... so that the assembled line expands as required to the full width of the mold." 3

In phototypesetter techniques, generally, the space-width considerations required in hot-metal justification processes are replaced by escapement values (i.e., "an escapement can be regarded as the distance between the left edges of the fields of two consecutive characters" ${ }^{4}$ ) and the physical displacements required in the optical system are controlled by escapement codes in the input tape.

"'The Photon machine can be driven by tape that contains a track specification (a track is half an annulus of characters), a lens specification (in effect a scaling factor), and then, for each of the characters to be printed, the sequence number (i.e. position around the disc) of that character, and the escapement (i.e., distance to be allowed between the left edge of that character and the next). Codes to change track number and lens size and to effect carriage returns are interspersed with the sequence number escapement codes where necessary." 5

Some differences between various available photocomposing systems are to be noted, however. If a Linofilm unit is to be used under computer driven control, complete horizontal justification information must be developed by the data processing unit and it must be recorded in such a way as to provide a paper tape that will control the Linofilm photocomposing equipment. This means that information with respect to exact character width values must be available to the computer so that character spacing and line length can be computed accurately.

If a Photon 200 unit with its built-in computer element is used, the adjustment of spacing between characters would be done by the Photon equipment: only the end-of-line signal need be included in the paper tape from the data processing unit. In this case the adjustment of character spacing to align left and right margins is a fairly simple operation even with large alphabets having considerable variation in type widths.

The character width information can be determined from a table during the character code conversion operation. In this way the detailed in. formation required in a Linofilm system could be 
developed by the data processing system in relatively little more time than that required to produce the lesser information required in a Photon system.

In both the case of hot metal casting and photocomposition where tapes are used to operate the equipment, it is obvious that keyboards and tape codes must provide for the insertion of controls to indicate length of lines, relative spacing, quadding, and the like, specifically including an approximate line-width-count for justification purposes. The latter usually requires a complex keyboard with built-in calculators to count the widths of the characters, compute the space remaining in the line and the number of interword spaces used, thus keeping track of the accumulative line deficit.

It is to be noted further that "since different typefaces all have different set, or width values, it is necessary to look up the width value of each character in the selected font and subtract that width value from the total remaining space in the line being set. Individual width cards or magazines which are inserted in the keyboard device are supplied for each type font. The keyboard device also keeps track of the number of interword spaces which have been inserted in the line. These word spaces may be expanded from a minimum to a maximum value thereby providing a justification range of some latitude for the keyboard operator. ${ }^{\circ}{ }^{6}$

In addition to the pioneering Friden LCC-S Justowriter, a number of keyboard-tape punching systems having calculators for brass matrix widths are commercially available, including Robotype, Perfoset, Linomatic, Lino Quick, and others. ${ }^{7}$

A recent device of this type is the DI/AN Controls Inc., punched tape keyboard for high speed typesetting. It is reported to produce "justified, corrected copy on punched paper tape, which controls automatic typesetting machines at rates up to 700 lines of 11-pica newspaper type per hour and 400 characters per minute in book production".

Control functions typically encoded into perforated tape are exemplified by reference back to Figure 19,9 showing the teletypesetter code format. Thus, for example, the quad functions are for use on linecasting machines which have automatic space fill to the margins. The "quad center" code will cause the centering of a short line of text, as in a heading, and will automatically generate equal amounts of blank space to the left and right margins. "Quad left" positions the line to the left and puts all of the blank space to the right, and "quad right" puts all of the blank space to the left.

"Space band" is the normal space used between words. Each one is an adjustable wedge. After the line of matrices (casting molds) and space bands are assembled, the space bands are driven in tightly to fill out the available space, and to equalize the spaces between words in that line.

\footnotetext{
6 Sparks et al., 1964, p. 166

7 See Duncan. 1964. p. 141.

${ }^{8}$ Data Processing Magazine 7, 5 (February 1965).

${ }^{9}$ See page 19 of this report.
}

If additional space or blank areas are needed, then "thin space", "en space", or "em space" can be selected. Em space is the width of letter " $\mathrm{m}$ ", en space is half an em, and thin space is one third of em. Note that in TTS code space band is coded with a single hole in track 3 , and thin space is a single hole in track 0 .

The function "add thin space" is 0 and 3 , and it causes selection of a space band and a thin space at the same time. Thus if it is discovered that the space bands are not adequate to fill out a line, then the perforator operator can go back and punch "thin space" over each space band code in the tape.

"Em leader" and "en leader" are dots used to fill out lines in tables where blank space is not desired.

"Return" resets the the justification counter or printers on the tape perforator. "Elevate" delivers the line of type for casting and also feeds a line of paper if a printer is used with the tape perforator.

The widths of the various characters used in TTS systems are shown in Table 2. Note that all figures (numeric digits) are 9 units wide. This facilitates the composing of aligned columns of numbers.

TABLE 2. Character grouping of unit system matrices (UNIVERS).

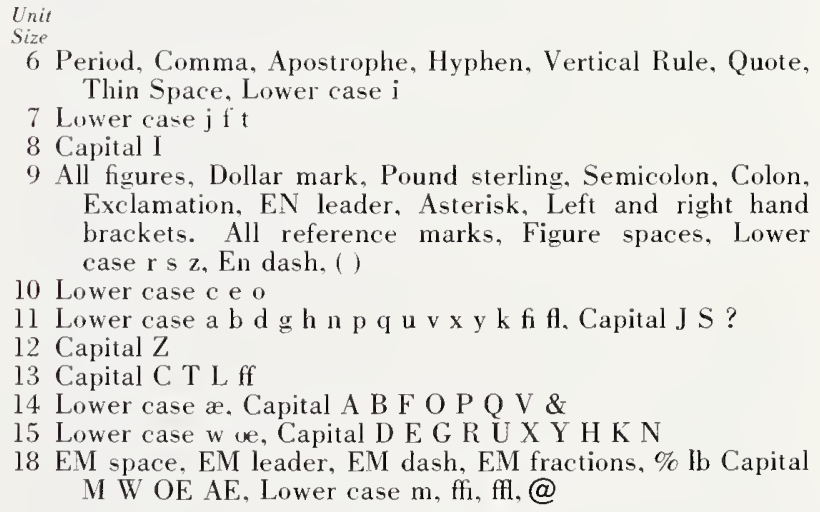

9 All figures, Dollar mark. Pound sterling, Semicolon, Colon, Exclamation, EN leader, Asterisk, Left and right hand brackets. All reference marks, Figure spaces, Lower case $r$ \& $z$, En dash. ()

10 Lower case c e o

11 Lower case a b d $g h n p q u \vee x y k$ fi fl. Capital J S ?

12 Capital Z

13 Capital C T L ff

14. Lower case æ. Capital A B F O P Q V \&

15 Lower case w ve, Capital D E G R U X Y H K N

18 EM space, EM leader, EM dash, EM fractions, \% lb Capital MW OE AE, Lower case m, ffi, ffl,@

Source: TTS Perforator Operator's Manual.

\subsubsection{Equipment and Techniques for Horizontal Justification}

The simplest solutions to problem of right-hand margin justification are (1) to ignore it completely, as in most ordinary typescript, or with respect to arbitrary line lengths in KWIC and other indexes produced by computer printers, (2) to limit justifcation to that which can be achieved by purely automatic means, such as the use of anamorphic 
lens stretching and compression of lines to be reproduced and (3) reliance on strictly manual decision-making.

One technique to improve page space utilization is that of the use of the principle of the anamorphic lens: "use of optical stretching or compression to alter the horizontal dimensions of a line of type without affecting the vertical dimensions... [Examples are] the Optype ... the Cape Grapha. tron ... the NHM Cartoprint automatic camera system". "This technique has also been claimed to achieve justification by purely mechanical means, in the case of the French-built Optype, as follows: "This, by a system of distorting photography, will stretch or compress individual lines of unjustified material until it justifies . .."'n

The methods employed for semi-automatic and automatic justification and word-hyphenation (as required) range from machine calculation of space usage within the line and display of the word in. volved in oversetting for operator decision to fully automatic modes of operation in general purpose computers used to drive copy reproduction devices on either an on-line or off-line basis.

From the manual decision case to the fully automatic systems, several different categories of increasing machine sophistication are available. In computer-driven, automatic systems, the principal methods used to date involve dictionary look-ups. application of pre-supplied 'logical' rules for hypention, look-up of pre-stored probability values for hyphenation between various letter pairs or trigrams, and combinations of these methods. Typically, each method within the available range offers advantages and suffers disadvantages.

The least sophisticated methods, of course, are those of machine "deference" to operator decision whenever a given line cannot be justified by appropriate automatic adjustments of interword and intraword spacing. Two quite different approaches to the man-machine interaction solution are exemplified by the so-called discretionary-hyphen system and by the Linasec devices developed by the Compugraphic Corporation.

"A manual technique for hyphenation, either with or without a computer system . . . was first tried by Louis Moyroud. . . This technique requires the keyboard operator to insert a discretionary hyphen in every long word. The computer or output printer can then utilize or disregard the discretionary hyphen during the actual process of completing horizontal justification. Although the use of discretionary hyphens is estimated to increase keyboard operation time by 2 to 5 percent and storage requirements by 2 percent the simplicity of the technique is somewhat attractive, especially for noncomputer output printing systems." "12 The obvious disadvantage, of course, is

\footnotetext{
10 Duncan. 1964. p. 148.

"O'Dunnell, 1961, p. 151.

sparks et al.. 196i. p. 184
}

the requirement for many decisions and many keystrokes that will not be used.

Operation of the Linasec 1 at Clay, Ltd., has been described by Boyd: "If no word space falls in this (justification) area, the word by which the line is overset is displayed on the cathode ray tube.... When this happens there are three possibilities: firstly, hyphenation by any of the left hand group of buttons at the appropriate point; secondly, if the word is unsplittable it can be removed completely to the next line, in which case the interword button is pressed; thirdly, if both hyphenation and interword have been tried and the word still remains on the screen, it indicates that the remaining 40 percent of band expansion is not sufficient to fill out the line. The monitor then presses the bell button followed by the interword button. This puts a caster stop code in the output tape and add-thin space codes can be punched in by hand or the thin spaces manually inserted on the casting machine." 13

Of the Linasec II device, Duncan remarks: "In the case of the Linasec 11. two functions are paramount. The output tape is coded correctly for the character widths so that no tight or loose lines result, and no redundant codes, and, more important, hyphenation is performed by manual selection on a monitor tube face. Compugraphic, the makers of Linasec, are convinced that this is a more economic solution at present than computer hyphenating routines, at least in applications where time is not the consideration it is in newspaper plants." 14 The newer Linasec is aiso reported to have an increased speed (from 45 to 100 characters per second) and to provide improved scope display and correction features. ${ }^{15}$

\subsubsection{Semi-Automatic and Automatic Hyphenation}

If a requirement for right-margin-justified lines in the printing or reproduction of textual and bibliographic material has in fact been established, then the problem of hyphenation will almost inevitably arise. The sole practical exception to date is the computer-compiled KWIC index with arbitrary line-length truncation and 'wrap-around' principles so that such horrors occur as:

"HEROIDAL WAVE EQUATION PROGRAMMING FOR
FINDING CHARACTERISTIC VALUES OF SP"
"EAN FUNCTIONS OF N VARIABLES USING A SINGLE
MAGNETIC CIRCUIT/STRAIGHTFORWARD WAY OF
GENERATING ALL BOOL"
"IGNMENT METHOD FOR ASYNCHRONOUS SEQUEN-
TIAL SWITCHING CIRCUITS A STATE VARIABLE ASS"
"CHNIQUES IN THE LOGICAL DESIGN OF SWITCH-
ING CIRCUITS RELAY CIRCUIT DESIGN TE"

Note that these examples were taken from just a few pages of Youden's Computer Literature Bib-

\footnotetext{
"1: Boyd, 196.5. p. 26.

it Duncan. 1964, p. 143.

is Morton. 1963, p. 99.
} 
liography, for which the 120-character line format should give rather better results than the twocolumn formats often used. On page 119, the following word breaks occurred at the right end of the title line:

$$
\begin{aligned}
& \text { "PROGRAMMin"[G] } \\
& \text { "COHE"[RENCE] } \\
& \text { "MANUFA"[CTURING] } \\
& \text { "ARB"[ITRARY] } \\
& \text { "C"[HARACTER] } \\
& \text { "COMPONEN"[TS] } \\
& \text { "OPE"[RATING] } \\
& \text { "CLA"[MPED]." }
\end{aligned}
$$

The generally poor typographic quality of such computer print-out indexes in all respects of typographic composition is probably a major factor in deterrents to their use. even when their prompt availability has been hailed as a major new bibliographic breakthrough.

The lack of any regard for 'proper' hyphenation is thus an extreme in the case of the KWIC index. Even for newspaper column composition, where reader tolerance for numerous typographical errors both intra- and inter-line is self-evidently high, concern with respect to improper and misleading hyphenation is obviously important. Such examples of least-tolerable 'goofs' in machine hyphenation include: ' 'han-gover', 'fig-hting', and 'Goodyear" ${ }^{17}$ or '" 'lam-plight', 'ons-laught' and 'however" "18 What might happen with words like "therapist" is all too obvious.

As we have noted, syllables (hence, proper hyphenation) depend on sound rather than letter combinations. It is not possible to develop a set of rules based on small sequences of letters that will invariably result in proper hyphenation [Bafour, 1961]. Ledley and Wilson, in a study of the potential use of computers for the publication of Index Medicus included a subroutine for automatic hyphenation, using one of the possible computer techinques. The resulting program, although fairly lengthy, would make many incorrect hyphenations, even on commonly used words. Ledley and Wilson could have reduced the number of incorrect hyphenations by enlarging their program, but the technique could never be made infallible.

As many as 35 different approaches to this problem have been taken by suppliers of computerdriven typesetting systems. ${ }^{18 a}$ In general, however, the various approaches may be grouped with respect to reliance on logical rules, dictionary look-up,

18 W. I. Youden. "Computer Literature Bibliography 1946 to 1963", National Bureau of Standards Miscellaneous Publication 266, U.S. Government Printing Office, Washington. D.C.. 1965, 463 p.

17 Experience with IBM 1620 at the San Francisco Chronicle as reported in "Signs of the Future?", 1964. p. 88.

18 Jitra routine for computer hyphenation of English, reported by Duncan, 1964 156.

${ }^{182}$ Cf. "The (R)evolution in Book Composition ... 11, 1964. p. 46: "International Business Machines alone has worked on more than 35 approaches to word division. Radio Corporation of America has at least two very different systems in operation," and Blondeau, 1963, p. 84: "In one of our laboratories we have written and developed over 35 approaches to the simple problem of grammatival word division and hyphenation." probability tables, and combinations of these principles.

Automatic hyphenation techniques were first explored by Bafour, Blanchard, and Raymond in France beginning in $1954,{ }^{19}$ with emphasis on the application of logical rules to words of the French language, but with some consideration also given to English. Bafour discusses how it is possible for a computer to determine the hyphenation at the end of a line:

"The information previously supplied to the computer enables it to recognize and distinguish the different letters of the alphabet, the justification spaces, punctuation signs, numbers, etc. The internal circuits are also able to add up the size of the letters and spaces, as they are coded, and to compare the total with the fixed point reached would require expansion of the spaces between the words (or between letters) beyond a tolerance fixed in advance (and always capable of being modified). It is able, at the same time, to recognize if a division between two words is possible or not. If it is not, it is necessary to search within a word for a possible cut. The empirical rules for the French language have been established and effectively proved. It is not possible to enumerate them within the outline of the present description and we will give only the following incomplete summary of them:

Not to cut after less than two letters.

Not to cut so as to leave less than three letters.

Not to cut after a consonant followed by a vowel.

Not to separate certain vowel couples forming an inseparable doublet.

Not to cut after a vowel if it is followed by two consonants which do not form an inseparable doublet.

Not to cut before the letter y.

Not to cut before a punctuation sign.

Not to separate two numbers, etc."

One computer technique that could be used for hyphen minimization would consist of a repetitive application of a line justification routine. Each repetition seeks a better solution for the paragraph as a whole. The iteration would stop when a solution was found that required no hyphens, when the approximation goal has been reached, or when all possible solutions had been tested. After each iteration, the best solution obtained up to that point would be retained for comparison with future iterations. The adjustment of character spacing would be accomplished simultaneously with the minimization of hyphenation.

A computer dictionary look-up operation would provide correct hyphenation for a high percentage of the words in the dictionary. Some English words are hyphenated differently in different usages so even with a dictionary some errors would be expected.

19 Bafour, 1965, p. 194: Sparks et al., 1964, p. 184: Duncan, 1964, p. 152. 
A typical computer-controlled justification-hyphenation process proceeds as follows:

"As characters are received as input, a subtractive process begins, each character, symbol, and space being assigned a unit count. When the number of units exceeds the capacity of a line, the last word is dropped and three questions are asked:

1. Will the expansion of space bands (from a normal 10 units to a maximum 40) fill the line?

2. If not, will the addition of thin spaces suffice?

3. If not, will an en space fill it?

If the answer to all three questions is ' $N o$ ', the 301 goes to the hyphenation routine ...

"After selecting the maximum number of syl. lables to fill the line, the computer returns to the space-band routine-in rare instances, resorting to letterspacing with the longest word, then to the addition of another space between words." 20

Operational examples of semi-automatic or automatic justification and hyphenation have been described in detail in the literature, for example, with respect to the Los Angeles Times, Perry Publications, and the Oklahoma Publishing Company.

At the Los Angeles Times, an RCA 301 computerbased system uses the dictionary look-up approach. Requirements involve not only the initial calculations for reporters' news copy, but also for re-justification (and, obviously, re-hyphenation as necessary) of copy received via wire service tape since, even though the latter may contain justification control data, it is typically for point sizes other than the 9-point type used by the Times. ${ }^{21}$

"The Times began with plans to store a dictionary of some $60-70 \mathrm{~K}$ words. When possible inadequacies and the high cost of this approach became obvious, the decision was made to switch to a logic system ...."22

The system developed is based, so far as practical, on grammatical rules for word division used in Webster's Unabridged Dictionary. More specifically, "the logic is based on the following principles. First, vowel and consonant patterns in a word are classified into one of four basic types. The computer then scans key letter sequences to see if they follow the rules governing this type. If so, an immediate solution is reached. For example, prefixes and suffixes which are commonly used can automatically determine hyphenation. Where exceptions are indicated, they are defined and analyzed by following special subroutines such as testing against letter sequence tables." 23

It is to be noted that the rules required to apply this hyphenation logic "occupy only 5,000 positions of the 20,000 positions of the computer's magneticcore memory, less space than would be required

\footnotetext{
23) Yasahi, 1963, p. 28

21 D. Ellis. remarks. "Computer Typesetting Conference Proceedings". 1965, p. 128.

Yasaki. 1963, p. 28.

23 Sparks et al. 1964. p. 184
}

to store 500 eight-letter words averaging two hyphens per word." 24

Success, in terms of agreement with first-preference Webster word-divisions, has been variously estimated as within the range of 85-95 percent accuracy. ${ }^{25}$ Toothman points to the occurrence of input spelling errors: "Obviously you are going to have some words that are in the dictionary and are not detected because there is a misspelling and so forth. These will be arbitrarily hyphenated. In most cases it will be wrong, and in that case it will be picked up by the proofreader." 26 The computer programs are owned by the Times but are available from RCA for use with the 301 computer. ${ }^{27}$

At Perry Publications in Florida, where an RCA 301 computer is also used, a dictionary look-up approach is the principal basis for automatic hyphenation. One of the reasons for this is the commitment to photo-composition techniques, where the relatively higher costs of page corrections (by comparison with hot-metal line-casting methods) dictate a better than 90 percent accuracy as a minimum performance requirement. Hence, the use of a dictionary involving approximately 40,000 root words of 5 or more characters each has been established. ${ }^{2}$

Some of the operational details that have been reported in the literature are as follows: "The justification process begins with the read-in of approximately $1 \mathrm{~K}$ characters. The input block is... [this] length, paragraphs of some 25 lines being considered the longest received at any one time... While the paragraph is being read in, the unit count of each character begins on a simultaneous mode. Following the space band after each word. a check is made to see if the line is justified. These checks continue until a line is filled, the 301 then branching to subroutines (if necessary) for the insertion of additional spacers and to the dictionary if hyphenation is called for." 29

For greater access speed and efficiency, the stored dictionary is divided between 4 tape units. "Since the RCA 301 computer can search tape in both directions, the tapes are always maintained at a given 'homing' position and the most frequently occurring words, based on their first letter, are located closest to the homing position on each of the 4 tape drives. An index to the positions of the dictionary on ... [these] units is maintained in core memory using the first 2 letters of the word as a key." 30

Thus. "the search begins with a determination of the first two letters in a word; this isolates the word to a particular block. .. . To save time, the most frequently used blocks are grouped around

\footnotetext{
24 Pay, 1963, p. 331 .

"s Thothman, 1963. p. 103: Sparks et al.. 1964. p. 181: "The (R)evolution in Book Comprosition." 1964. p. 47.

2k Touthman. 1963.p. 105.

27 Pas. 1963. p. 330 .

Ex "The (R)evolution in Berk Compesition ... 1\%64, p. 47-48.

23: Yasaki. 1963. 1..30.

su Sparhs ot al. 196t. 1). 183
} 
a middle, homing position. About 98 percent of the word, as a result, are accessible within one second. " 31

Moreover, "if the word being searched is not in the block, it is automatically hyphenated after the third, fifth, or seventh letter. ... Thus, all words which correctly break at these points have been purged from the dictionary." 32 Accuracies of up to 99 percent are claimed. ${ }^{33}$

These two cases represent. respectively, a logicrule approach and a dictionary look-up approach. A third method, that of pre-storing statistical probabilities for correct hyphenation depending upon observed letter sequences is involved in the Oklahoma Publishing Company operation, together with some of the "better aspects" of the logical and dictionary approaches. ${ }^{34}$ This system "utilizes two IBM 1620 computers with 20,000 characters of core memory, IBM tape readers operating at 500 characters per second, and tape punches operating at 50 characters per second . . .

"The hyphenation program begins with an edit of the word to determine the number of syllables, and in some cases determines the hyphen point, or the inability to hyphenate at a point. It next determines the probability of hyphens between any two letters in the word and hyphenates at the most probable point... Accuracy of 94 percent is reported for this program ..." 35

More specifically. "an analysis was made of 40,000 different words with their frequency in order, and the probability of a hyphen occurring before, between, and after every possible letter pair was figured. Then these probabilities were stored in the computer. When a word was to be hyphenated, it was scanned letter pair at a time, thus developing the probabilities of a hyphen occurring at the various points. The most probable points are selected as hyphenation points." 36

The Oklahoma example may perhaps represent a trend, at least in the United States, to the combining of various techniques in a single system. Thus, "the various approaches being taken today seem to be converging toward a combination system-a basic logic program supplemented by a small exception dictionary of words that cannot be broken properly by the rules... Opinions differ, however, on how large the exception dictionary will have to be to achieve this goal. One manufacturer believes it can be reached with 2,000 words, while another maintains it requires 12,000 words." 37

The principal advantage of dictionary look-up approaches is that accuracy can arbitrarily be improved, although short of 100 percent perfection, by the inclusion of more and more words in the

\footnotetext{
31 Yanahi. 1963. p. 30 .

Jbid.

"The IRevolution in Bork Composition . . " 1964. p. 18.

Blondeau, 1963, p. 87.

3 Sparks et al. 1964, p. 184.

Blondeau, 1463. 86-87.

$3 \pi$-The (Rtevolution in Bowk Compusition..., 1964. p. 48.
}

dictionary store. The corresponding disadvantage is, of course, the size and cost of memory storage space required.

Two relatively recent developments envisage the use of large-capacity random access storage devices for this purpose. Announced in 1965 was "a low-cost computer for automatic hyphenation and justification in the publishing field by Automatic Media Systems, Inc.. Boston, Mass. It features a 2 millisecond memory storage of 3.3-million bits, enough for all the 325.000 English word entries in Webster's dictionary. The machine is pre-programmed, and comes with two paper tape readers and punches. Accuracy is reported to be better than 99 percent of all lines hyphenated." 38

Also, at National Cash Register, it is claimed that "we can, if we are pushed, store the whole of the big Webster in one CRAII deck with the hyphena. tion points. in practice one would use two." 39

However, it is by no means clear that even such large dictionaries are fully adequate. Special requirements are imposed by high incidences of proper names in newspaper work, by highly specialized practices in scientific nomenclature, ${ }^{40}$ and by the emergence of new words and word-combinations both in English and in other languages.

Some representative opinions with respect to these considerations are as follows:

(1) " If a dictionary look-up is resorted to then it must be continuously updated and arranged so that the most quickly available items are those most used." 41

(2) ". . A dictionary of some 600,000 words might ultimately be needed. It is possible to compile a hyphenation dictionary of these words for insertion into a random access device. However, the number of words involved and the constant appearance of new words makes this an expensive procedure if it is to be sufficiently comprehensive to be effective." 42

(3) ". . . The problem as regards the Dutch language is not finite, because we make any compound word we want; we string words together without spaces. as is the case in certain other languages such as German. . . ." 43

The last of the above quotations reflects the experience of the Mathematical Center in Amsterdam. Corstius reports further: "We have to . . . [use] a logical process. .. . We tried our solution on 50,000 running words in normal Dutch text. We achieved 99.5 percent accuracy. . . Most mistakes were made in words which were not Dutch but English or French. They often occur in Dutch text. The machine cannot see which language it is." 44

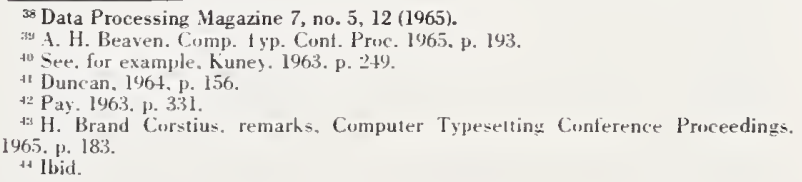


Results reported for other automatic hyphenation programs include successful experiments by Mitra using Ferranti computers ${ }^{45}$ and trials of the TRWFairchild system: "Right now, we are running at 95 percent accuracy. . . " 46 Other programs are the technique developed at the Mathematical Cen. ter in Amsterdam, used for example, in the preparation of an anniversary publication of the Dutch Computer Society; ${ }^{47}$ lBM experimental programs for French, German, and Dutch, ${ }^{48}$ and continuing work at CAE (Compaginie Europeane d'Automatisme Electronique) in applications of the Bafour-type logical system to French, English, German, and ltalian. ${ }^{49}$

"Programme de justification de textes" in the November-December 1964 issue of 'Electro Calcul provides a current account of the CAE logical system for hyphenation of French language texts, as tested with Flexowriter input, giving only three errors in over a thousand word divisions.

An unusual and interesting application of the Dutch justification-hyphenation routine is the automatic typesetting of Braille. "We are going to use the same programme for a TTS machine. For a Braille printer, which is being installed in Delft, Braille characters are rather large, there are only about 30 on a line, so you have to hyphenate frequently, the same programme will be used." 50

"At M.I.T., Assistant Professor Dwight Baumann has developed a high speed Braille printer using a computer as an integral part of the system." 51

In view of the obvious difficulties of achieving proper justification by any set of fixed and invariant rules, it is not surprising that a number of different approaches to semi-automatic and automatic justification and hyphenation have been taken: for example, as previously noted, in a single organization as many as 35 different methods have been tried. Nevertheless, "at the moment, the clash between the strict logic of computers and the largely illogical basis for word division in the English language present programmers with one of their most pressing challenges. In a very real sense, it is the hyphenation problem that continues to exert a restraining influence on the more rapid spread of general-purpose computers beyond the newspaper industry." 52

One special problem with respect to techniques for automatic hyphenation involves the question of ligatures (spatially overlapping character pairs or triples) in a given composition style or type face design. "Provision for hyphenating between characters forming ligatures is not included in many programs currently in use. Programming this re-

\footnotetext{
${ }^{45}$ Duncan, 1964, p. 154.

te F. S. Angell, remarks, "Computer Typesetting Conference Proceedings," 1965. p. 180 .

${ }_{47}^{180 .}$ "Computer Typesetting Conference Proceeding," 1965. p. 183.

t* Duncan, 1964, p. 157

: Bafour, 1965.

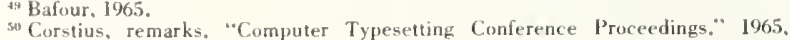

p. 184 .

${ }^{31} \mathrm{Kehl}, 1965$, p. 25

s2 "Computerized Typesetting," 1964, p. 5.
}

quirement is more complex than a first glance would indicate ... [for example, after a hyphenation decision 'of-ficial', it may be necessary to] re-analyze the second $f$ and the $i$ to recombine them into a different ligature". 53

One service center organization, ROCAPPl, has proposed a working solution to this problem as follows: "What you have to do . . . is to perforate 'ffi' normally, forget that ligatures exist altogether, and let the computer programme in its final stage determine, (a) whether or not that ' $\mathrm{ffi}$ ' is in the lower case, (b) whether or not this line involves any letter spacing, and (c) whether it is in a font where you have a ligature available. This is in the [Rocappi] system now." 54

The difficulties associated with semi-automatic and automatic hyphenation are manifold. The first of these is that natural languages typically require word splitting at points of phonetic stress which do not always agree with usual syllable boundaries. ${ }^{55}$ Moreover, the syntactic usage of word forms that are orthographically identical may dictate different hyphenation points, as in the case of "pres-ent" and "pre-sent".56 Some breaks are to be preferred to others largely because of providing greater context expectancy to the reader for the remainder of the word to follow: thus "conferencing", "conflag-ration", "conduct-ance" and "conversa-tion" are certainly to be preferred to the rigorously correct splitting after a prefix. There are also, regardless of the authority used, many exceptions to the usual rules of syllabification.

The existence of variations in both rules and conventions of preferred practice raise the even more fundamental question of what criteria are to be used in judging "proper" hyphenation, as Duncan remarks: "Our first difficulty was to find an authority on what was right and what was wrong, and we found those in English texts confusing and, in some cases, contradictory . . .57

Other corroborative testimony on this difficulty includes the following:

(1) ". . First dictionary preference hyphenation. The only problem that you get into there is which first dictionary, because dictionaries don't agree, either." 58

(2) "One of the first problems to be faced in developing hyphenation programs is what standards to use-word division differs from one dictionary to another. However, a consensus seems to be developing for use of Webster's Unabridged dictionary." 59

(3) "lt must be emphasized that there is no error-free technique for hyphenating a word,

\footnotetext{
5.3 "The (R)evolution in Book Composition . . 11." 1964. p. 48. ${ }^{54}$ C. Barber Comtyp Conf Proc 1965 p. 164.

55 See Duncan. 1964. p. 156.

s6 Statement of C. Barber. Computer Typesetting Conference Proceedings, 1965. p. 107.

${ }^{37}$ C. J. Duncan. remarks. Computer Typesetting Conference Proceedings. 1961. 135 .

SN Tootliman, 1963.p. 103

"The (R)evolution in Book Composition . . " 1964, p. 46.
} 
either automatically or manually. Human keyboard operators do not have perfect recall for the content of Webster nor do they always refer to a dictionary when in doubt." 60

The last point, namely, the incidence of at least some human error in manual hyphenation raises two further questions for system design: whether, on the one hand, errors introduced provide either quantitatively or qualitatively a serious enough situation to warrant expensive equipment and costly proofreading and whether, on the other hand, hyphenation problems might be avoided entirely.

A practical question is the number of lines in running text that must be hyphenated in order to achieve effective horizontal justification.

Operating experiences reported in the literature include the following data:

(1) "The [Los Angeles Times] system generally involves one hyphenation per 7 lines of text." 61 Moreover, in view of the 85 percent average accuracy expected, "lines incorrectly broken are then reset manually after the type has been cast. If the computer hyphenates one line in eight, an accuracy of 85 percent will mean resetting 30 lines in 800 (two lines must be reset for every word division error), or 3.75 percent of all lines cast.," 62

(2) "The magazine Printing Technology, published by Britain's Institute of Printing, Ltd., recently published an article set via the Newcastle installation, in which about 4 percent of the lines set were hyphenated." 63

(3) "We have found that decisions on 8-point on 12 pica will run about one for every five lines. We have also found that when running 8-point on 15 pica, the decisions drop tremendouslya decision every twelve lines." 64

It is to be noted that the situation changes favorably and rapidly the greater the column width and hence the more opportunity for variation in both inter- and intraword spacing. For text page composition without column breaks it is reported that: "On long-measure book work, in the area of 25 picas, manual operators may hyphenate one line in 10, while initial computer experience has produced a record ranging from one line in 100 to one in $1,000 . " 65$

Quantitatively, then, the problem may not be too severe. From a qualitative standpoint, however, the ludicrous cases will of course leap to the eye and be well remembered to the detriment of recollection of the proportion of lines requiring no splitting or ending in correct hyphenations.

A final solution to the problems of automatic hyphenation is therefore that of returning to the "ignore" solution of avoiding or at least minimizing

\footnotetext{
60 Sparks et al.. 1964, p. 185

61 Ibid.. p. 184

62 "The /Rjevolution in Book Composition ... 11," 1964. p. 47.

6.7 "Computer Hyphenation. . " 1964, p. 45

in Morton, 1963, p. 100.

".5 "Compuler Hyphenation

$1964,0.45$
}

word breaks by iteratively resetting a number of lines. This solution has been advocated by Duncan, on the basis of the University of Newcastle experience, as follows: "The first programs were used in processing texts to procure some information, until then totally lacking, on the percentage incidence of word splits in various widths of lines when set within certain spacing limits, and an early decision was taken to try to avoid word breaks as often as possible, i.e., to minimize the difficult part of the problem - hyphenation.

"Within certain limits it appears that word breaks and wider word spacing are interchangeable, and by the processes programmed at Newcastle it is possible to avoid word breaks completely in the wider line lengths common in quality bookwork . . .

"There is already evidence that a computer can do what the better compositor-typographer desiresgoing back over several, perhaps many, lines and adjusting spacing to improve evenness and closeness and avoid awkward splits." 66

It is further reported that "In Britain, experiments in computer justification at the University of Newcastle upon Tyne have attempted to limit the splitting of words to an absolute minimum. The computer is programmed to hold an entire paragraph in its memory until the last line is set. If a line must be hyphenated to justify it, the computer will first try to reset the previous lines in the paragraph to eliminate the word split. If it can"t eliminate the split, it will then set the paragraph with the fewest possible splits." 67

Further evidence of the probable merits of the no-hyphenation solution is offered by the following: "One newspaper recently tried an experiment in which an entire page was set without hyphenation. Loose lines were justified by adding all required space between words. Printed samples were dis. tributed to a number of editors and executives on several papers, and their comments were requested. The experimenters were surprised to discover that no one seemed to miss the hyphens, and that added space between words aroused almost no comment." 68

On the other hand, while "some doubt has been cast upon the value of horizontal justification by psychological studies designed to test its effect on reading efficiency, it has a psychological value rooted in long tradition." 69

In addition to such psychological or aesthetic factors, the gains from introduction of semiautomatic and automatic justification and hyphenation techniques include increased productivity in the keyboarding of copy, gains in the efficiency of hotmetal casting equipment, and type density improvements with less waste space and thus less page and paper cost. These gains, combined, can produce

\footnotetext{
66 Duncan. 1964. p. 154-160.

67 "Computer Hyphenation . . ", 1964, p. 45

68 Ibid.
} 
both "significant cost reductions and faster publication schedules." 70

With respect to the first of these factors, it appears that the keyboard operator works faster and makes fewer errors when stroking unjustified and unliyphenated output copy, than when producing justified tape and making manual hyphenation decisions. Representative operational data reported in the literature include the following:

"Although figures published as to the economics of unjustified tape perforation have varied widely, it is generally conceded that a net productivity increase of 30 to 40 percent can be reasonably anticipated. With keyboarding a major focal point in typesetting costs, this saving can be significant." 71

"We have developed statistics at Perry Publications that indicate they get about 45 percent faster production by having a typist type unjustified paper tape as against typing with the justified." 72

"A 30-40 percent increase in the production of punched tape is claimed by the [Los Angeles] Times using a 301."73

"The most difficult saving to estimate accurately is also the most important: that is, increased keyboard efficiency. Before we committed ourselves to Linasec we made some initial trials with one particular operator, and this showed that an increase from 10,000 ens an hour on justified work to 15,000 ens an hour on unjustified work was possible. In practice, what we hope to achieve is somewhere around 20 percent to 30 percent average increase over a roomful of perforators." 74

Results of Oklahoma Publishing Company's use of 1620:

"1. A computer copy tape requires about 80 percent of the strokes that are necessary for normal teletypesetting operation.

2. The operator perforates about 30 percent faster since he isn't concerned with justifica. tion and hyphenation decisions." 75

"Rocappi, Inc., has estimated that a TTS perforator operator who theoretically could produce 15,000 characters . . . per hour of justified tape would be able to set 25,000 characters . . . of unjustified computer input tape." 76

"The studies revealed that a skilled tape punch operator may be expected to meet production of 2,560 lines per eight-hour day, based on 80 percent productivity of an operator punching and justifying at a working rate of 400 lines per hour. It was estimated that a relatively skilled typist producing copy at 80 words per minute, 6 words per line, might be expected to net approximately 5,120 lines per eight-hour day, based on an 80 percent productivity." 77

\footnotetext{
"Kuney, 1963, p. 2.2.

7 "Computerized Typrsetting," |964, p. t.

72 Toothman, 1963, p. 106.

${ }^{73}$ Yasaki, 1963, p. 28.

Boyd, 1965, p. 31

Blondeau, 1963 , 86

*The (R) 1 os, p. 86

Donk Composition

Morton, 1963, p. 96.
}

A second gain, in some cases, that of increased efficiency of the linecasting equipment itself, has been hailed as unexpected: "An unexpected ad. vantage has been less down time on the linecasting machincs. Since the computer is not apt to set lines either too loosely or tightly, the linecasting machines can operate at steady high speeds without jamming or undue wear." 78

Boyd comments to much the same effect as follows:

"Another saving that computer justification . . . brings is on the linecasting machine itself. Tape processed by Linasec [I] is almost entirely free from either slack or tight lines and also the lines where the keyboard operator has forgotten to press any end-of-line key at all. . . ."

"A tight line in a caster causes a stoppage and loss of production out of all proportion to the fact it is just one tight line. . . ."79

Finally, to the extent that computer justified input tapes minimize machine stops, they also "reduce accordingly the need for monitors and machinists on line casting machines." 80

\subsection{Corrections, Updating, and Merging}

Still another advantage of the introduction of semiautomatic and automatic methods to the composition operations is the provision for making corrections, updating, and merging with greater efficiency, less cost, and higher speed. The problem of the high costs and difficulties of making corrections to standing type, as a result of author changes in proofreading has long been recognized as a special problem in scientific and technical primary publications. It has become so severe that, as Duncan claims, "A number of scientific periodicals, particularly American ones, and a number of book publishers, can only continue in business by refusing all corrections subsequent to acceptance of final typescript." 81

In the pioneering Bafour system it was anticipated:

"Further it is possible to obtain at the same time as the coded tape, a typed copy which is an exact indication of the contents, including the registered signals. It is also possible to produce, after this initial operation, a second tape of the modifications or additions which one wishes to incorporate in the initial setting, by recording them with respect to the initial signals.

"At the input of the computer we only have a reader for two tapes of which the second, much shorter, serves to control modifications of the signals of the first in the desired places."

In particular, even the deletion of a single word, the substitution of a longer word for a shorter and vice versa, or the addition of one or more words

\footnotetext{
$7 \times$ Goodwin. 1964, p. 34

${ }^{79}$ R. Boyd, remarks, Computer Typeseuting Conference Proceedings, 1965, p. 37. so 'asaki, 1963.p. 29

\$1 Duncan. 1964.p. 124
} 
to a single line typically affects far more than the resetting of that one line. "Even if the problem is only that of correcting an improper hyphenationdecision, at least two lines must be reset.

The introduction of photocomposing techniques not only did not help the text revision correction problems - it made them worse.

"In the composition of newspaper text, photocomposition has never been widely employed because of the page revision problem. Part of this problem results from the fact that it takes so long to phototypeset corrections and get them into new plates for printing." 82

Systems involving punched paper tape output as well as hard copy have also been notoriously awkward to use for the insertion of corrections and changes unless tape merging devices have been provided. Otherwise, the operator must reproduce the original tape with careful watch for indicated positions for change and, upon reaching such positions, manually step the tape through character by character until those requiring change occur, then punch their replacements into the new tape and resume machine reproduction until the next correction indication is reached.

The two such systems usually credited with pioneering introduction of calculators for line space counting were also innovators with respect to tape merging and editing functions. Thus, "the Friden Justowriter ... pioneered built-in calculation of the justification-spacing parameters and of tapeediting procedures," 83 and "Robotype is the first automatic merging system specifically designed for printing. The keyboard in this case is a modified IBCI typewriter. It produces a justified tape and also the hard copy on prenumbered paper." 84

Duncan reports further that: "Semiautomatic methods of keying-in corrections were demonstrated quite conclusively by Bafour in his 1958 examples and have been further developed in the Robotype auto correction system in which the input is in coded blocks with tagged lines. " 85

Today, "Linofilm, Robotype, Justowriter and Photon among others all provide facility for merg. ing tapes used in their typesetting systems." 86 The Intertype computer is another example, offering both readers and punches which can be controlled in various merging operations at rates up to 100 characters per second.

A special feature of such automatic tape merging systems may be the provision of means for automatic detection of at least some errors which might be introduced by the keyboarding-punching-processing operations. For example, "The computer produces paper tape and, as it is punching, it adds up the values of each code, just the binary values of every single character, whether it is a character

*z V. M. Corrado, remarks, Computer Typesetting Conference Proceedings, 1965. p. 221.

*3 Duncan, 1964, p. 146

Broyd, 1965, p. 32.

ws Duncan. 1964, p. 164

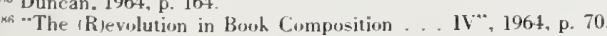

or a function. At the end of each line the programme puts that total out on the tape. At the end of a page, the programme tells the operator it has finished the page, and the operator takes this piece of paper tape and immediately puts it straight back into the computer again ... When this tape is right we take it to our typesetting device, which has ... an output punch ... [that] punches a second tape. At the end of the page we compare the two tapes ... If there is an error, the computer prints out a little note saying "line 4, 7 th character might be an error' ". 87

It is to be noted, therefore, that: "Merging operations offer an obvious and immediate area in which computers can automate the composition process. Merging is involved in many composition jobs-everything from inserting corrections in new type to revising and updating previously printed material." 88

Working with either source manuscripts or revised editions of previously printed material, extensive insertions and deletions may be necessary. The new advanced techniques offer such capabilities as the following:

"This article reports a new approach to the paper tape updating problem, which involves the use of a high-speed electronic digital computer. A computer program that is called the ESI system has been written to enable the 709 computer to revise paper tapes for a Friden Flexowriter ... The facility to update Flexowriter tapes with a computer... implies the facility to produce updated Photon tapes.

"The style of the editing instructions used with the ESl system is illustrated by the following examples ....

"Delete and close from the fifth paragraph through the second occurrence of a paragraph beginning with the name 'Alice' . . .

"Start a new line with the last sentence in the seventeenth paragraph.

"Start a new paragraph with the last two words in the fourth line of the seventh paragraph . . ." 89

Similarly, at Rocappi, it is claimed that "we have the ability to accept input from any known conventional paper tape perforator. We can justify and hyphenate. We have correction procedures to insert and/or delete material at will. In other words, we can kill from the third word on line 90 to the eighth word on line 737 and insert one word, two lines or the Encyclopedia Britannica." 90

Still other prospects are for automatic asseribly by computer of completed galleys, plus editorial changes, deletions and insertions into appropriate page make-up for the completed article or book. This would also involve the merging-in of footnotes, titles, section headings and the like.91 Looking

\footnotetext{
s: C. T. Ross, remarks, "Computer Typeselting Conference Proceedings", 1965 p. 94 .

"The (R)evolution in Book Composition ... Ill", 1964, p. 56

Bs Barnett and Kelley, 1963, pp. 99-101.

${ }^{90}$ C. Barber, remarks, Computer Typesetting Conference Proceedings, 1965, p. 108 s) See Ohringer, 1964, p. 313.
} 
ahead to the multiple processing capabilities of general purpose computers Barnett and Kelley suggest further:

"This approach could be generalized to deal with source material on several input tapes. The niaterial would be selected, organized, and edited in accordance with an edited text description that the computer read from another tape. The description of the material to be copied could use several different addressing systems. These could involve page numbers, chapter and section numbers, document date or author, chapter and section names, and combinations of these and other forms of reference. The ordered selection of material could be augmented by further computer operations, such as sorting, in the production of the final output." 92

As of today, however, while automatic aids to horizontal justification and hyphenation have been extensively tried, with varying degrees of success, the problems of vertical justification and page composition have been attacked sparingly and, usually, on a strictly experimental basis. Thus the conclusions reached in a relatively early study are still highly pertinent today. From "Report on the Study and Investigation for the Design of a Lexical-Graphical Composer-Printer System,"93 in the words of reports of the Mergenthaler Linotype Co., we find the following (page 7):

"The page composition and page make-up functions involve the ordering of alpha-numeric characters into lines of type, the arrangement on makeup of these lines into the text columns of a page, and the allocation of space on the page for related illustrations, all in accordance with specified page formats."

In that study, an examination was made of the most flexible conventional techniques, methods, and equipment for page composition and page make-up, employing hot metal casting of either individual characters or complete lines. Hot metal methods were selected because their extreme flexibility offers a "worst case" from the point of view of duplicating the functions in an automated system. The results of that investigation of hot metal procedures is summarized, in the list of functions given above, which omits manuscript preparation, galley proofs, art work, dummy make-up, proof reading, preparation of table of contents and indexes, and operations leading to printing.

Quoting again from that report (page 7): "To those unfamiliar with the graphic arts processes that lead to the printed page, these results serve as an eye-opening revelation of the complexity of the tasks involved in producing a product that is so casually accepted and taken for granted. To the initiated, these results serve as explicit confirmation of the fact that the incorporation of page composition and page make-up capabilities in a lexical-

${ }_{92}$ Barnett \& Kelley, 1963, p. 108

${ }^{93}$ Mergenthaler Linotype Corporation, 1963, p. 7. graphical composer-printer system is a complicated and formidable task."

Similarly, with respect to a specific job undertaken for Random House, Freiman reports that "on the Linofilm, using the normal set-up with conventional ready-made grids, we discovered that we would have to average about 10 grid changes per line. This made the Linofilm absolutely out of the question because of the slowness thus engendered." 94 The same commentator also suggested, however, that the Linotron with its very high speeds and multiple-pass capabilities would provide an adequate solution to such problems.

Merging in of corrections, insertions and deletions has already advanced so far in newspaper composition work that in the Los Angeles installation, for example, it is claimed that: "Much of the savings generated by computerized typesetting at the [Los Angeles] Times is based on computer insertion of editing changes." 95

Still another advantage of new facilities for automatic or semiautomatic insertion of editorial changes and corrections lies in the area of variously styled editions, revisions, and re-issues of material ranging from training manuals to textbooks and even to the automatic combination of several texts..$^{96}$

Some typical claims with respect to primary publications or secondary publications, or both, are as follows:

"One application is the conversion of source documents into any kind of a composition system, either directly from authors' manuscripts or from previously printed documents into a new format such as hard-cover books into paperbacks." 97

"There is a useful by-product for the book printer in a continuous tape system: that is, that a book once set can be produced in a variety of type faces from the original or corrected tape." 98

"If a new edition is required, then the original tape, with a correction tape, and the new style instructions can be re-submitted to the computer. This provides a substantially new product, but one in which the cost of keyboarding is probably only $10 \%$ of the full cost." 99

"A second type of re-issue involving only alterations in style arises from the use of the computer generated tape with compatible but relatively minor changes in the photo-composing machine. Just exactly what is possible will depend on the output machine used, but a simple example would be composition in $8 \mathrm{pt}$. on $9 \mathrm{pt}$. body to some suitable reduced page width and depth in place of the original which was $10 \mathrm{pt}$. on 11 pt. body composition." 1

Finally, there is the prospect stressed by Corrado that material available in computer-usable form but

\footnotetext{
${ }^{94}$ R. A. Freiman, remarks, Computer Typesetting Conference Proceedings. 1965 pp. 230-231.

ss "The (R)evolution in Book Composition ... 111", 1964, p. 56

Barnett and Kelley, 1963, p. 106.

${ }^{87}$ Merz, 1964, p. 85

gल Boyd 1965, p. 25

Duncan, 1964, p. 162

1 lbid.
} 
not presently reproducible with typographic quality may in the future be re-processed:

"We are preparing a master typography program for a general purpose computer. . . Input to this master typography program can be existing magnetic tape. For example, there may be a tape standing today that was meant to be run on a 1403 line printer. With a minimum number of editorial insertions for what we call an insertion program, the information on that tape can be made sophisticated enough to permit the master typography program to provide all the information for the Linotron." 2

Barnett and Kelley point out, further, that: "In general, the production of a revised publication requires typesetting and proofreading processes that repeat the expense and time delays of the original production, even though the changes may affect only a small fraction of the characters in the text. Changing a few words here and there may cause quite considerable changes in line and page breaks, and 'stripping in' can be very awkward and time consuming." 3

\subsection{Vertical Justification and Page Composition}

Just as close, even spacing desiderata are achieved by line-by-line horizontal justification, so also are vertical justification and page make-up considerations important in terms of the page as a whole.

"The advent of computer actuated type-setting and photosetting devices makes it all the more important and advantageous to focus attention on the problem of finding the most effective formats for the composition of the printed page." 4

One possible computer solution to the problems of horizontal justification and minimized hyphenation requirements has already been reported: that of holding the contents of a set of lines in memory and iteratively re-trying line justifications to improve the entire paragraph or page. This solution is closely related to problems of vertical composition, whether of column or page, especially avoidance of "widows" (i.e., left over at the bottom of a paragraph or page, or carried over to the beginning of the next page).

In this connection, some idea of the difficulties of providing broadly applicable "machine rules in the automation of compositor functions is provided by the example of the programmer who "told the computer that a widow of three characters was possible-whereupon the computer produced a line containing only a period and two closing quote marks." 5

In book composition planning at the Westerham Press, for example, Maclntyre reports: "The items we were interested in controlling were word-spacing,

\footnotetext{
${ }^{2}$ Corrado, 1964, p. 116

${ }^{3}$ Barnett and Kelley, 1963, p. 99.

'Eugene Garfield, panel discussion, Markus, 1962, p. 23.

"The (R)evolution in Book Composition ... II," 196r. p. 47.
}

letter-spacing, removal of widows, short lines, and multiple hyphenations occurring sequentially." 6

Page composition operations at ROCAPPI (which had, in collaboration with RCA, produced over 50 samples of book pages as early as 1963) involve such operations as the following: "The function of Phase III is to make up the matter from the galley output into pages, putting in running heads, folios and chapter headings, picking out footnotes and references, putting in captions, and so on, and at the same time eliminating widows, short lines at the top of a page, hyphenations breaking over a page and other typographical horrors." 7

Another aspect of the page composition problem involves the question of requirements for switching between character sets, beyond the single-run capability of the specific equipment.

With respect to relatively early Linofilm experience, for example, it is reported that:

". . Since point size may be changed only at the beginning of a line, it is not possible to switch from an A grid, which can produce point sizes from six to twelve, to a B grid, which has point sizes from twelve to twenty-four. If it is desired to vary point size within a line, it would be necessary to make two passes, changing point size and film advance to accomplish the desired result." 8

In the case of scientific and technical text involving frequent insertion of mathematical and other special symbols, the problem is particularly severe. For example, Croucher remarked on some of these difficulties, involving several different systems at the London computer-typesetting conference as follows: "We found that we averaged 200 grid changes per page, on Maths, on the old 88 matrix grid", and, "we found the Linofilm difficult because of the fact that we had to bring in practically all the 18 grids even on one page of mathematics." 9

The multiple-pass solution to the problem of changing fonts and hence enlarging the total character set repertoire is also suggested for more recent developments such as Linotron which, to an extent, trades character set size during a run for extremely high speed character generation. Thus, "in area composition ... you can do all of your Century, say, first. At the end of that pass you go back, and while you are going back, you can change the character grid to your Spartan, and then fill in all the holes with your Spartan. Consequently, the number of font changes is only as large as the number of different type faces on a page." 10

Prospects for progress include recognition that: "The computer can assign page numbers, allow predesignated spaces for inserting drawings, photographs or other graphic information, and can insert subject headings and column headings automati-

\footnotetext{
6 MacIntyre, Comp. Type, Conf Proc, 1965, p. 34

7 T. H. V Archer, remarks, Computer Typesetting Conference "Proceedings", 1965 p. 120.

"Graphic Composing Techniques", 1962, p. 22

${ }^{9} \mathrm{G}$. Croucher, remarks, "Computer Typesetting Conference Proceedings", 1965 p. 230 .

${ }_{10}$ Corrado, 1964, p. 114
} 
cally. By minute variation in the leading between lines, the computer can easily accomplish vertical justification so that the bottom and top lines of all columns on the same page will be flush." 11

Barnett and his colleagues also point out that: "The problem of linearizing formulae is really a special case of the more general problem of linearizing two dimensional topologies. A scheme is being developed by the authors, that gives names to objects, and then compounds these names in expressions in which connective symbols and operators are used to indicate topological association, scaling and alignment." 12

"The aim to produce close even spacing throughout is not a mathematical ruling-in fact due to optical variations it calls for slight variations in spacing according to the shape of the encompassing letters, just as do the beginning and ends of lines with some characters or sorts, and some founts. This information can be fed into the computer's memory and used repetitively." 13

Other prospects promised in current computer programming developments include: "A further refinement of spacing concerns rivers of white. It should be possible to define them by a string of numbers or place labels. They can then be searched for, before they occur in the computer output, and possibly avoided.

"Fully automatic editing can be achieved using the type of special language created by Barnett for 'calling' the changes and for finding the correct place to make them using the text itself to indicate the "address'." 14

Finally, the problems of vertical justification and page romposition are related to the problems of area composition as in display advertising, composition of book jackets, and the interpolation of two dimensional graphic material. Barnett considers the latter to be far more difficult as such than those of page makeup:

"Page composition provides a descriptive problem that is less serious, as the subdivision of pages into rectangles of material that can be set as units usually is simpler than the corresponding subdivision of formulae. A simple notation can be used to describe a page by an expression which uses names for items of text, and a nested algebraic notation for the distribution of these, in adjacent rectangles of different sizes." 15

In terms of hardware developments applicable to many cases of page and area composition, one example is Linofilm.

"The Linofilm system ... embodies one important 'first' - the Composer, which is essentially a selective rephotographing device completely under the control of the layout operator. With this editing and re-arranging device the operator can do complicated display work with reductions

\footnotetext{
Sparks et al., 1964, p. 185.

Barnett et al. 1963, p. 270.

Duncan, 1964, p. 160.

14 Ibid.an, p. 164.
Bar.

Barnett et al.. 1963, p. 270
}

down to $2 / 3$ and enlargements up to $6 \mathrm{X}$. The keyboard has recently been modified to include mixing facilities ('Linomix') and editing simplified by a tape merging and reproofing device ("Tape Combiner')."16

"The primary advantage of the rapid display response time of a cathode ray tube is in the editing of texts where several sequential editing steps are required and where the editor cannot proceed with a particular step until he has seen the results of a former step... This rapid response capability is useful in page makeup where rewriting and rejustifying of text are required to fit text items in their allotted spaces." 17

\subsection{Two-Dimensional Composition}

Over and above requirements for a large, versatile character set, pre-prepared and available either directly or indirectly, many applications have need of a capability to insert within a printed page symbols and other material extending in both dimensions beyond the confines of a single line. In scientific and technical information processing, the most obvious examples are mathematical equations and chemical structure diagrams. The two professional societies most obviously concerned have therefore been studying these problems over the past several years.

At the American Mathematical Society use has been made of specially designed Photon discs and a pi-mat attachment for the setting of Critical Reviews of Mathematics. Subscripting and superscripting operations are readily available. At the American Chemical Society, Kuney and his associates have been exploring various approaches to the setting of chemical symbols, as well as other aspects of high-speed, higher-quality machine-aided composition, since 1958.

One obvious alternative in both the mathematical and chemical cases is to abandon the traditional symbols and to use alphanumeric names for them instead. "With the limited keyboard available on the present Flexowriter, most mathematical symbols and Greek characters must be essentially spelled out in abbreviated form. . . . This not only reduces the speed at which the input text can be transcribed but also results in tedious typing for the operators, thus increasing the possibility of errors." 18

The typesetting of such special symbols is claimed to be "one of the most difficult tasks in the modern composing room ... the setting of mathematical equations, chemical structural formulas, and other items that, although part of the text, differ from ordinary text. ..."19 It is also reported that, quantitatively, the number of two-dimensional insertions, from tabular data to diagrams and illustrations in scientific and technical text, ranges from $40 \%$ to $70 \%$ of the total text. ${ }^{20}$ Moreover, require-

\footnotetext{
${ }^{16}$ Duncan, 1964, p. 144.

${ }^{27}$ Buckland, 1963, p. 179

18 "Graphic Composing Techniques", 1962. p. 33

${ }^{19}$ Kraft, 1963. p. 8.

${ }^{20}$ Buck et al., 1961. pp. I-3.
} 
ments for manual setting of complex mathematical and chemical formulas are such that conventional typesetting facilities tend to become saturated. ${ }^{21}$

"The process of preparing chemical structures for printing purposes is complex, slow, and expensive. While much has been done using the characters available in cast metal type, such structures are subject to spillage and breaking of characters, particularly subscripts." 22

Photocomposition techniques have offered some new possibilities. Thus, Barnett reports of Photon developments:

". . Letters, digits, and other symbols to be used as subscripts and superscripts are available on many discs. . . The base lines of such characters are displayed with respect to the normal character base line, so that they are displayed automatically in the appropriate position with respect to the characters which they append". ${ }^{23}$

An example of Linofilm capabilities is provided in Figure 46.

As Feldman notes, "The recently introduced 'cold-type' typesetting machines [such as Photon and Linofilm] appeared to offer promise for the composition of chemical structures, because these machines do have the ability to go from one line to another before either line is finished. However, certain computations still have to be made in this process; there is also a backlash which becomes noticeable when a line is approached once from above and then from below." 24

As previously noted, work on typographic-quality composition by semi-automatic and automatic means began at the American Chemical Society at least as early as 1958. A first approach was the development of a Photon test disc providing 50 lines and angles with which to construct the characters proposed at American Cynamid for the typing of structural formulas. Using this approach, for example, benzene rings can be constructed which range from $1 / 4$ to 3 inches in height. ${ }^{25}$ Then, ". . . to develop compatibility with the input of the Army chemical typewriter, the proposed character design was extended to include:

1. Full-length single and double bonds.

2. Combinations of half-length straight and angle bonds for corners and inserting of alphabetic and numeric characters within the ring.

3. Offset single bond lines to be used to form double bond effects within the inner structure.

4. A complement of miscellaneous characters." 26

A report by the University of Pittsburgh research group working on the Chinese ideograph project exemplifies some of the difficulties but also points to some of the potentialities for other types of

\footnotetext{
U.S. Senate, 1960, p. 117.

32 Kuney and Lazorchak, 1964, p. 303.

${ }^{2}$ Barnett et al. 1962, p. 63.

* Feldman, 1964. p. 205.

${ }^{25}$ Kuney and Lazorchak, 1964, p. 304

${ }_{28}$ Ibid., pp. 304-305.
}

Q. Can formulas be set flush left, flush right or centered?

A. Yes. Quadding of the component lines in formula composition is the same as for normal text, so long as the start of each line is at the same reading (ems and units) on the Keyboard Unit indicator tubes. Likewise each line must end on the same indicator reading. These readings are determined by the cast-off line. The following examples show all three types of quadding

$$
\begin{array}{r}
\int d x F(x)\left[-x \frac{d}{d x} \delta(x)\right]=\int d x\left(x \frac{d F}{d x}+F\right) \delta(x)=F(0) \\
\int d x F(x)\left[-x \frac{d}{d x} \delta(x)\right]=\int d x\left(x \frac{d F}{d x}+F\right) \delta(x)=F(0) \\
\int d x F(x)\left[-x \frac{d}{d x} \delta(x)\right]=\int d x\left(x \frac{d F}{d x}+F\right) \delta(x)=F(0)
\end{array}
$$

Q. Can the formula be centered and the reference number set at the right?

A. Yes, as shown in the following example:

$$
\int d x F(x)\left[-x \frac{d}{d x} \delta(x)\right]=\int d x\left(x \frac{d F}{d x}+F\right) \delta(x)=F(0)
$$

$(32.0-27)$

Q. Can one character be superimposed over another?

A. The examples here show superimposition achieved by the same technique in text lines and in a display formula.

It can be done readily as this $\hbar$ in this justified line and also as shown in the formula below.

$$
=F_{u \cdot . .}\left(\frac{\hbar}{i} \frac{\partial}{\partial q^{\prime \prime}}, q^{\prime \prime} \cdot\right) \partial_{\left(q^{\prime}-q^{\prime \prime},\right)}
$$

FIn,URE 46. Linofilm mathematical setting examples.

stylized two-dimensional graphic material, such as line drawings.

Pertinent findings and observations include the following:

"The input writer is a modified 'Flexowriter' with a keyboard containing the strokes needed to form Chinese ideographs, and keys controlling the operational functions which place the fully formed ideographs in the filmstrip text. The keyboard also furnishes information to a code generator and to the logic system within the machine.

"The keyboard contains 21 basic strokes, 20 ideographic entities, and 11 punctuation marks. Each stroke has been assigned a letter of the alphabet. Special English alphabet key caps have been fitted so that non-Chinese operators can compose on the machine by using Chinese language copy that has been English-language coded in advance by Chinese-English linguists.

"Each stroke generates a 5-bit code which is fed into shift registers and then into a coincidence detector. The magnetic drum . . . has a capacity for storing codes for 10,000 ideographic characters. These characters are made of as many 
as 20 strokes... When coincidence occurs between the stroked character and the same character stored on the drum, a signal is generated.

"Employed in the optical system are an optical tunnel, a vidicon imaging lens and faceplate, and a photographic memory plate (called a character matrix) with a matrix illuminator, containing 160 groups of 16 ideographs each. This system transfers a replica of the character, selected by the operator and located by the logic system, from the character matrix to the input of the video system. The video system electronically scans that group of 16 characters on the character matrix which contains the one demanded by the operator. It then selects the one required and presents it to the operator, on a display kinescope ... for verification.

"A character, generated on the exposure kinescope . . . is transferred by means of the "photograph' key on the keyboard to the optical system and is imaged by means of the lens system onto photographic film.

"When the operator strokes a sequence that defines more than one ideographic character, the first character recognized at the coincidence detector will have associated with it a special pulse indicating ambiguity. In this case, a maximum of four characters can be displayed.

The operator can select the desired character by stroking keys on the keyboard that are especially marked to indicate the quadrant of the display kinescope where the desired character is shown.

"An insertion vidicon camera is built into the machine to allow the insertion of characters not contained in the machine vocabulary.

"If a required character is not present in the machine vocabulary, the operator can place his copy, or a document containing the desired character, on an insertion tray. When the 'insert' key is depressed, the insertion vidicon is activated and will scan the document or other material, and the vidicon system will reflect what it sees on the display kinescope. The operator can relocate the document until he has the display he desires. The zoom lens of the vidicon is then adjusted to display the character in the desired size. $" 27$

\subsection{Specific Photocomposing Equipment}

The possible development of keyboards specifically designed to feed photocomposition systems has been under consideration for a decade or more.

A special case of two dimensional symbol com. position is that required for typing or printing Chinese ideographs. The Lin Yutang typewriter is an example.
"On 8 June 1955 a contract, supported jointly by the Army and the Air Force, was awarded for the development of a prototype machine . . . delivered in December 1959. The Sinotype had a basic vocabulary of 2,333 ideographs and was of an electric-pneumatic-mechanical design. It proved to be quite slow in operation, too sensitive to the shocks of field use, and difficult to maintain." 28

Radio Corporation of America has developed a Chinese ideographic photocomposer for the U.S. Army Natick Research Laboratories. A modified Flexowriter provides keyboard input for 20 complete symbols, 21 strokes, and 11 punctuation marks.

Codes generated by the keyboard address a memory drum, which in turn directs the illumination of portions of an optical character matrix. The character is viewed by a vidicon camera tube and displayed on a verification oscilloscope before photographing. Special characters can be inserted by means of an insertion vidicon. The machine vocabulary is 2500 characters, expandable to 10,000 characters.

Characters are photographed on film in $1.0,12$, 18 , or 24 point size, in a page format of vertical columns set from right to left, or horizontal rows set from left to right.

Methods for reproducing chemical structure information by special typewriter and keyboard or by high speed computer printout have been explored by Jacobs and Feldman at Walter Reed, by workers at American Cyanamid, by Crane and associates at Eastman Kodak, and by Waldo at Monsanto.

In the Army chemical typewriter developments of Jacobus and Feldman, for example, a set of character and conventions of usage are first developed. "Then, as the structure is typed, a tape is perforated with the codes of the characters typed, and the codes of the coordinates of the position of the characters on the typewritten page. . . A computer program, rearranging the output of the ... typewriter so that chemical structures can be typed out line-by-line, was written by Harold L. Manceaux. Grouping according to the $y$-coordinates brings together all characters that appear on the same line; these characters are then spaced proportionately to their $\mathrm{x}$-coordinates. By ordering the $y$-coordinates sequentially, a tape containing a lineby-line output is obtained. Such computer-produced tapes are suitable for driving tape-controlled typesetting machines." 29

It is planned that encoding and typing of structures in accordance with the Army chemical typewriter scheme can be computer-processed to obtain a tape to drive the Photon for setting of chemical structures. ${ }^{30}$ Holm points out, also, that:

"Mechanical input is possible through the Walter Reed Army Institute of Research type-

2* U. Pitt., 1964, pp. 1-2.

${ }^{20}$ Feldmin, 1964, pp. 206-207.

30 Kuney and Lazurchak, 1964, p. 305 


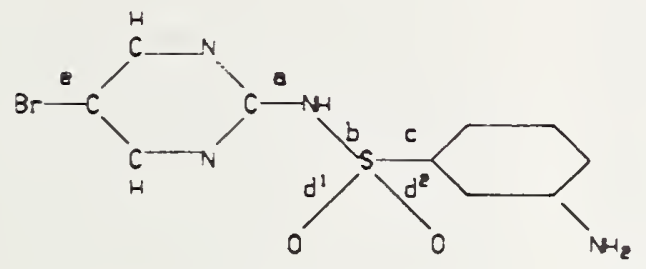

$$
\begin{array}{ccc}
a=1.42 & d^{\prime}=1.50 & \angle \mathrm{ab}=113^{\circ} \\
\mathrm{b}=1.75 & d^{2}=1.50 \text { (ass.) } & \angle \mathrm{bc}=100^{\circ} \\
c=1.80 & p=1.90 & \angle d^{\prime} d^{2}=132^{\circ} \\
\text { (errors uncer:ain) } &
\end{array}
$$

C.S.: $\left\{C_{1}\right\rangle$

$2=0.24$ (Siructure daminated by heavy aloms)

$\mathrm{C}_{32} \mathrm{H}_{3} \mathrm{OO}_{2}$

Lanostenyl iodoaceiate

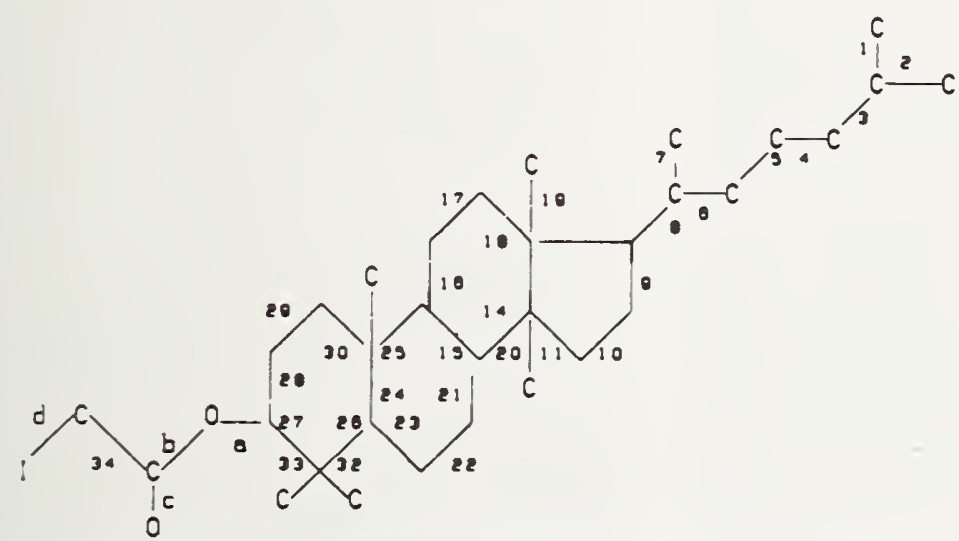

The erystallographic observations are consistent with the chemical

reptesentation of the skeleion shown above. NOTE - The numbers refer io bonds not io aions.

$\begin{array}{lllll}1=1.64 & 7=1.52 & 13=1.64 & 19=1.55 & 25=1.47 \\ 2=1.64 & 8=1.59 & 14=1.67 & 20=1.39 & 26=1.39 \\ 3=1.41 & 9=1.66 & 15=1.38 & 21=1.50 & 27=1.53 \\ 4=1.40 & 10=1.66 & 16=1.61 & 22=1.54 & 28=1.52 \\ 5=1.56 & 11=1.56 & 17=1.69 & 23=1.54 & 29=1.52 \\ 6=1.62 & i 2=1.56 & 18=1.51 & 24=1.39 & 30=1.57\end{array}$

$3 !=1 \cdot 44$

$32=1 \cdot 51$

$33=1.55$

$34=1.52$

$0=1 \cdot 33$

$b=1 \cdot 24$

$c=1 \cdot 19$

$d=-2.02$

C.S.: $\left\{C_{1}\right\}$

Fic,URE 47. Output of Technical Text Compiler.

writer or through optical scanning. However, the capability exists today to take any of these input forms and transform them within computer memory ... if the input generates a unique and unambiguous form. . . " 31

Under U.S. Army sponsorship, also, General Electric investigators and personnel of the Redstone Scientific Information Center have developed an experimental Technical Text Compiler System, utilizing an IBM 7094 computer and the S-C 4020 display capabilities. Input can be accepted from either line-at-a-time devices such as the Flexowriter or from the Army Chemical Typewriter providing X

3) Holm. 1965. p. 162. and $Y$ coordinate information (Burger, 1964). Sample output, as of December 1964, is shown in Figure 47.

Kraft, in 1963, described the Mergenthaler "Diagrammer" as follows: "Mergenthaler's Diagrammer is a prototype device by means of which an operator can project any one of 400 symbols onto a viewing screen, move the projection across the screen to any one of a series of positions, and expose the symbol onto the corresponding position of a D-size (or smaller) photographic film or paper.... In the prototype Diagrammer, the entire symbol complement is composed of electronic symbols and their associated nomenclature. . . ." 32

\footnotetext{
${ }^{32} \mathrm{Kraft}, 1963$, p. 13.
} 
In a Diagrammer model demonstrated in July 1965 a basic character set of 256 symbols is available to the operator at any one time. These are stored on glass slides for optical projection onto a viewing screen. The operator is frec to move a selected symbol about and to change its size as required.

Two other examples of computer output capabilitics are the generation of printout of annotated flowcharts and weather maps, as follows:

"The present approach was to define an algorithm capable of one-pass operation a small (IBM1620) computer, using a Calcomp digital incremental plotter online to produce the annotated flowchart. The use of a digital plotter capable of operation under program control seems largely to have been overlooked in past considerations of automated flow charting. Two advantages are immediately apparent: (1) solid-line unrestricted definition of chart symbols; and (2) an essentiatly unlimited character set. . . "33

"Another approach to handling computer output is to scan and transmit microfilms generated by devices like the General Dynamics S-C 4020, the Data Display dd-80, or the scanning converter built by Burroughs Michigan Laboratory to replace the 30 by 30 inch printer plotters now used with the Weather Bureau's 7094 computer to produce weather maps for facsimile distribution." 34 Another surprisingly early direction of experimental investigations, stemming in large part from the interests of the Rome Air Development Center, has been the question of intermixing textual and graphical material, in high typographic quality output, especially for such areas as output from mechanical translation programs where it is desirable to interpolate equations, charts, drawings, figures and other illustrations including half-tone representations of photographs in the appropriate positions with respect to the machine-translated text. A series of three reports highlight projects at Syracuse University (Buck et al., 1961): IBM ("Graphic Composing Techniques," 1962), and Mergenthaler (Mergenthaler, 1963).

Some of the aspects that are of general and continuing interest include the following:

Syracuse Report, 1961. This survey outlined various system proposals and extensively reviewed the then available technology and equipment, including proposed developments and modifications. Pertinent details, such as planned modification to provide better typographic quality of the S-C 4020 and the CBS character generator, early versions of the Alphanumeric and Videograph systems, and descriptions of equipment not now available (DACOM, Electrada), have been cited as appropriate elsewhere in this report. Two further examples, of peripheral interest to the main topic of this report but reflecting future prospects for two dimensional composition and interpolation of graphic with textual material have to do with a potential mix of character recognition techniques for text and the identification and location of graphic material to be copied or stored for later interpolation in proper page makeup. First, it was noted that:

“. . The microfilm containing text and graphics can be used in an optical projection system. The shutter of the projection system may be controllert by signals established by the text reader to indicate the presence and extent of graphics. . . ." 35

Secondly, with respect to the proposed FMA "Text Discriminator and Reader Programmer", it was reportech that:

"This equipment, as proposed, would be an electronic read-in device incorporating . . techniques to distinguish text from graphics. . . Some of the more promising techniques are:

"l. Frequency response differentiation between text and graphics. Text is characterized by sharp edge and maximum contrast while graphics contain slow changes and all shades of gray.

"2. Recognition of special marks associated with graphics and equations. Generally minus and plus signs will be present in equations, thus providing unique symbols which can be recognized easily. Also the density of marks and equations is not uniform as in the text."

IBM Report, 1962. This report concerns the exploratory work undertaken to tie together the AN/GSQ-16 (photoscopic disc) machine translation system with the special IBM 704-Mergenthaler Linofilm system also used by Barnett. More specifically, the project objectives were to process graphic data through the translator in order to reproduce such data in the printed output, and to improve the typographical quality of the translated-text output.

Some specific details are as follows: "The composing rate is only about 60 words per minute. . . There are usually quite a few font changes per page, and each font change requires several seconds. . . Most articles have a number of untranslatable items, such as charts and pictures. It is necessary to leave blank film in the output, so that the items may later be pasted in their proper positions. A page or text with no font changes or pictures can be printed at about 110 words per minute.

"The translated information would contain a quad space, or tabulate, character between the two sections of the information [i.e., text, and graphics to be interpolated]. This causes the program to quad left the information which is in the output area at the time of the quad space character. Then a character is placed in the output area which sets a zero film advance, and the remaining information is assembled and written out with a quad right end 
of line command. This causes the second section of the information to be written on the same line as the first part. When the line has been written, the normal film advance is restored. and the program continues.

"If the information is to be centered on two or more lines. the total width of the information to be centered has been accumulated in the 704 memory. This value is divided by the number of lines on which the information is to be centered, to get an approximate width for each line.

"If graphical material interrupts a sentence. the sentence must be completed to the nearest punctuation mark (except for a comma) before the necessary $\mathrm{Par}$. and Tab. instructions can be given for the space allotment. Control or edit instructions must not interrupt a sentence or idea. If edit information is inserted in the midst of a sentence. an improper translation may occur.

"If the information is to be centered on two or more lines, the total width of the information to be centered has been accumulated in the 704 memory. This value is divided by the number of lines on which the information is to be centered. to get an approximate width for each line. . . .

"Nost of the work done to prepare the translated information for the Linofilm unit is done by an IBM 704 computer. . . A number of instructions have been added to the 704 which, although not necessary, simplify the working program. For this reason. the particular 704 program which is used is not directly compatible with another 704." 36

Mergenthaler Report, 1963. This report, and its appendices, carry forward still further the question of present and future prospects for "Lexical-Graphical Composing-Printing" (LGCP) systems. Specific consideration is given to machine-controlled composition. reproduction and/or material ranging from line art illustrations. tabular matter and mathematical equations to screened halftone illustrations. Thus: "The tonal scale of the illustration is simulated in the printing process by making the dot pattern structure on the printing press plate a function of the tonal gradations of the original illustration. Thus the dark areas of the original illustration are represented by a dot pattern in which the dots are relatively large and with little white space between the individual large dot areas. . . .

"The output graphic must be a dot pattern structure in which the distribution of the dots over the image area represents a faithful reproduction of the continuous tone input image. It should be noted that since the dot pattern structure does not appear in the input graphic, it must be created within the LGCP system." 37

Other pertinent comments in the literature on present and future prospects for two-dimensional composition and interpolation of graphic material in machine-processed text include the following: First, a negative reaction: "The keyboarding re-

35 "Graphic Composing Techniques". 1962, pp. $7,14,17,18,29$

${ }^{37}$ Mergenthaler. 1963, pp. 13-14. quired to code a complex formula would probably be greater, or at least no easier or less expensive. than direct setting without the intervention of a computer." 38

Secondly, optimistic, including the incorporation of color: "The practical application of such displays as chromatron photo chromic dyes, coupled with such reproduction means as thermoplastic recording or smoke printing will usher in an era of true electronic printing in all the flexibility we have come to expect in printed communication." 39

Thirdly, some specific difficulties: "If the original is screened halftone or a combination cut, however, it is essential that the enlarged output have the same size as the original: inasmuch as it is impossible to change the size of the picture without changing the size of the screen. which will damage the appearance of the halftones.

"Because it is a highly skilled operation, considerable difficulty can be anticipated in incorporating automatic screening into the Lexical-Graphical Composer-Printer. However, such recent developments as the Logelectronics projector may make it feasible." 40

Fourthly, implications for man-machine collaboration: "The paste-and-cut method for transferring equations. graphs. etc., could now be replaced by the post-editor indicating which figure from the source text should be placed where in the output if optical printing techniques are used." 41 Again, “. . Special mathematical symbols which do not appear in the tape as it comes from the printer are added ... on the character display tube by use of the light pen which we have attached to our PDP 4 computer." 42

Fifthly, the prospects for drastically reduced image-storage requirements, with a reasonable facsimile reproducible upon demand. "Much work is under way to store pictorial representation, such as the chemical structure in packed coded or binary form, with the reproduction of the original pictorial form upon request either as a display on film, or printed." 43

Finally, with respect to both present and reasonably predictable future technological developments, we may agree with Duncan's conclusion that: "There appears to be no real technical barrier to the simultaneous or practically simultaneous composition-imposition of both text and graphics." 44

\section{Documentation Applications}

In the broad field of documentation, generally, the introduction of high speed semi-automatic and automatic composition techniques will undoubtedly have its first and greatest impact in the field of

\footnotetext{
38 "Computerized Typesetting", 1964, p. 5.

3 Herbert, Ohlman. Panel discussion, Ifarkus. 1962, p. 25.

40 Kraft, 1963, pp. 7-8.

41 Reitz, 1964, p. 278.

42 Ohringer, 1964. p. 313.

t3 Holm, 1965, p. 166.

+ Duncan, 196\%. p. 135.
} 
primary publication especially that new genus. the publication of computer-compiled and computergenerated materials. This new genus ranges from numeric tables through directories and revised editions of training manuals to text and tables of reports automatically generated by machine reference to and sclection from stored data.

Applications to computer output also include the complementary operations of conversion of text of computer-usable records for storage and subsequent search and selection, on the one hand, and use of computer-usable text for multiple purposes, on the other.

Applications in the area of the more conventional primary publications range from typesetting of text for articles, books and newspapers through the issuance of revised or different editions to the merging in of editing changes at early manuscript or proofing stages and the merging of "core" material with special local variations.

In the area of secondary publications, indexes whether automatically, semi-automatically or manually prepared, announcement journals, computerized book and card catalogs, accession lists, bibliographies generally and demand bibliographies resulting from machine-searches, abstract journals, automatically prepared abstracts, shelf lists, accession lists and output of translations of material from one language to another (whether by man or machine) are all technically promising candidates. A new impetus might be given to the "current contents" concept if cooperating journals could transmit text of their table-of-contents pages by wire to a center which would assemble and reproduce them preserving the original typography if desired.

Special-purpose or more sophisticated applications will probably extend from today's experiments on composition of relatively stylized twodimensional materials such as chemical structures, mathematical equations and drawings to the page composition of both text and graphics, and perhaps eventually to the results of man-machine interaction via display consoles and light pen insertions.

In the remainder of this section we will consider examples of some of these applications which are either in practical production, have been demonstrated experimentally, or have been extensively investigated. Before discussing applications to primary and secondary publications, newspaper and book composition, specialized bibliographic aids and relationships to mechanized storage, selection and retrieval systems and service center operations, however, we will consider prototype general purpose programs for computerized typesetting.

\subsection{General Purpose Computer Programs}

The first of the general purpose computer programs developed for a variety of possible documentation operations is that of Barnett and his associates at the Massachusetts Institute of Technology.

\subsubsection{Barnett and Others at M.I.T.}

The pioneering work of Barnett and his associates at M.I.T. runs a gamut from proper presentation of computer-compiled and computer-generated primary publications and to secondary publication in many forms precisely because its intent is general purpose. Generality is achieved in allowing Flexowriter, punched card, and other forms of input and in program 'packaging', while output versatility (709-driven Photon processing and later, 7090) is as is illustrated in Figure 48.

This was produced from the input record, in part as follows:

[indn77d121s24stl, , 36cnxs l]

EXCERPT FROM ALICE IN WONDERLAND

[nlls 18]

December 6, 1961

[sp4st2, 10, 36st3, 11, 36st4, 12, 36st5, 13, 36st6, $14,36 \mathrm{st} 7,15,36 \mathrm{st} 8,16,36 \mathrm{st} 9,17,36 \mathrm{st} 10,18$, $361 \mathrm{sl} 4 \mathrm{dll} x \mathrm{xs} 2 \mathrm{rl}]$

[sc19scl9] Fury said to

[xs3] a mouse, That

[nlxs6] he met

[xs7] in the

[xs8] house

[xs9] [sc19] Let us

[xs7] both go

[xs6ls12] to law:

[xs5d19]I[ d d l ] will

[xs3] prosecute

[xs2d19] you.

[nlxs3dll] Come, I'll

[nlxs4] take no

[nlxs5] denial:

[nlxs7] We must

[nlxs81sll] have a

[nlxs9] trial[se47]

[nlxs10] For

[xs7]really

[xs6] this

[nl] morning

[nlxs8ls10] I've

[xs6]nothing

[xs5] to do.'

[xs4] Said the

[xs2] mouse to

[xsl] the cur,

[nlxs3] [sc19] Such a

[nlxs41s9] trial,

[xs3] dear sir,

[xs2] With no

[xs2]jury or

[xslls8]judge,

[nlxs2] would be

[xs2] wasting

[xs2] our breath.'

[xs31s7] [sc19]I'll be

[xs2] judge,

[xs2] I'll be

[xs2]jury,' 
[xsl] Said

[xslls6] cunning

[xsl] old Fury:

[xs2] [sc19]I'll try

[xs2] the whole

[nlxs3ls5] cause.

[nlxs4] and

[xs3] condemn

[xs3] you

[xs3] to

[xs3] death.",

[en]

Note should be taken first of the intermix of "program" (i.e., input, processing. and output instructions) and textual data and secondly of the extent of the total input occupied by precedence and special coding. This may involve a high initial keystroking penalty, as mentioned elsewhere.

The anticipatory control subroutine may be of particular significance in integrated publication processes since, as Buckland points out: "Page, volume, and issue numbers are needed for journal citations in an index. This data is not known until the typesetting and page and journal layouts are complete and would therefore. have to be derived from the composition information, whether or not a computer is used to prepare the type." 1

A specific application example is the production of the Union listing of Chinese Communist scientific periodicals prepared by the M.I.T. Libraries. Barnett reports: "The source material has been punched on a Flexowriter, with special symbols representing diacritical marks in an arbitrary correspondence. Certain types of input item are delimited by carriage returns, tabulations, slashes, periods, and combinations of these and a few other simple criteria. The relevant control codes are inserted for new lines, indentations, columnar alignments, changes of type and diacritical marks, by a program that is specific to this application." 2

In general, in the M.I.T. program, ". . The computer is used to set material. organize and to impose elaborate formats mechanically onto material that is keyboarded in a convenient and casual manner, thus avoiding the extensive clerical work and precise typesetting that would otherwise be necessary . . "3 and, "It has been our objective to develop a general purpose program which could deal with a considerable variety of photocomposing problems that were defined in a suitable input language. A language and the corresponding program have been developed that take care of features which are common to many typesetting situations." 4

Some specific details of the computer-composer programs, at various stages of development, serve to illustrate both programming challenges and diverse application potentialities. First, an initial

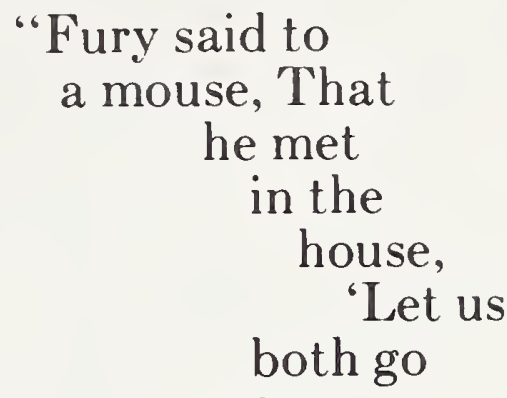

to law:

I will

prosecute

you.

Come, I'll

take no

denial:

We must

have a

trial;

For

really

this

morning

I've

nothing

to do.'

Said the

mouse to

the cur,

'Such a

trial,

dear sir,

With no

jurs or

judge.

would be

wasting

our breath.

- I'll be

judger.

I'll be

jurr:

Sitid

cumning?

(4) lin ling:

olli ir:

He. whole

anis:.

inulo.ill

in

diatlit...

Figure 48. Burnett program output.

Buckland. 1965. p. 8.

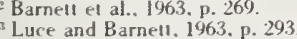

- Barnell el al.. 1963. p. 264 
control code sets certain composing parameters such as page width and page length, sets the type style by table lookup, and selects the type size (usually, the smallest lens size available). ${ }^{5}$ This initial code also controls the setting of body lead (distance between the base lines of two successive lines) and specifies vertical spacing. ${ }^{6}$

The input code, for example, for Flexowriter material contains both the style codes which by suitable conversions will control the selection of the appropriate disc track number of the Photon and other control codes, specify justified or unjustified lines, flush left, centering, or flush right, and, for justification, specification of the criteria to expand or compress interword spaces. ${ }^{7}$ Essentially, such Flexowriter input "is treated as a continuous stream of information which is split into lines in the output by reference (i) to line width and justification considerations, (ii) to control codes in the input that specify the beginning of new lines explicitly or implicitly, and (iii) to paragraph breaks." 8

Next, the input codes are converted by computer program (with the various control codes in the material automatically calling up the appropriate program sub-routines) to a composition-controlling code.

"The disc level, lens size, sequence number, escapement, carriage return and vertical space codes constitute the $W$-code, that is produced by the photocomposing programs and is written on magentic tape, from which punched paper tape is produced off line, using an improvised magnetic to paper tape converter." 9

In terms of the internal computer processing, "The status of the setting process at any instant is recorded by some 70 parameters. These are stored in a 'status' array. . . Whenever a parameter is changed, a record is kept in a 'backtrack' array of the position in the status array of the parameter that has been changed, and of the superseded value. If overset occurs before an interword space is encountered, the contents of the backtrack array may be used to restore the status of the setting process to that which was current when the previous word-end was encountered." 10

It is to be noted further that, in the Flexowriter input case, "The input subroutines for Flexowriter material deal with backspacing and error correction codes in the tape, both in the representation of the text and within interspersed control information. ..."11

With respect to vertical justification and page composition it is noted that: "The "run-on" instruction ... is used to remove a paragraph break, by bringing the first word of a paragraph in the unedited text into a position two blanks beyond the period ending the previous paragraph. If the end of the previous paragraph was too close to the right

\footnotetext{
${ }^{5}$ Barnelt et al., 1962, p. 6I.

"Ibid, p. 64.

[bid, p. 62: 1963, p. 266

[bid, 1962, p. 6].

'Barnett et al., 1963, p. 265

10 lbid, p. 268

"lbid, p. 267
}

margin, the first word of the 'run-on' paragraph would be brought back to the left margin and the blank line between paragraphs closed out ..."12 and "If page breaks are not specified, a text of arbitrary extent is split into pages of the appropriate length by the photocomposing program."13

Programming complexities and attempts to maintain adaptability to future, more sophisticated requirements are further exemplified as follows: "The evolution of the programs that effect the . . . (input code to composition code) conversion has consisted largely of the provision for an ever increasing variety of format requirements." 14

". . A further subroutine that deals with control codes is then called. . . This is switched by the control code to a subroutine that deals with the particular code that has been encountered. This segregation of the effects of individual control codes facilitates continued expansion of the set of codes with a minimum of recompilation." 15

"It can be seen that in some types of applications, certain combinations of control codes would occur repeatedly in the input, if interspersed coding were employed. For this reason, it is convenient to define macros, for an individual job, by suitable statements that precede a text in the input medium, and then to use the names of the macros, between square brackets in the text, whenever necessary. At present 25 macros may be defined for concurrent use ... (incorporating) up to 12 individual control codes." 16 "The nested structure of the program can be extended to include further layers, and this will be done to deal with problems of page composition." 17 "Expansion of the program to deal with larger units of text in a coordinated manner (e.g. providing page justification, arranging 'run arounds' to leave space for diagrams that will be visible when the relevant portion of the text is read) will require increased provision in the program for retrospective modification of the $w$ string that has been formed." 18 "Delayed action codes will make it possible to specify elaborate formats in which changes in style and so forth occur at points, such as line endings, which cannot be anticipated until the spacing considerations have been determined by the $p$ to $w$ conversion."19

The work of Barnett and his M.I.T. colleagues has obvious applicability, because of its generality, to service bureau or service center operations where many different customers may send their work for processing. Duncan says: "The practical result of the M.I.T. team's work to date are best exemplified by the Typrint and Tabprint manuals which editorial staff use to order up their work from the computer." 20

\footnotetext{
12 Barnett an Kelley, 1963, p. 104.

${ }^{13}$ Barnett el al., 1962, p. 65.

"S Barnett et al., 1963, p. 264

15 lbid, p. 268.

16 Barnetl et al., 1963, pp. 268-269.

13 Ibid. p. 268.

${ }^{11}$ Ibid, p. 268.

19 Ibid, p. 269.

${ }_{20}$ Duncan, 1964, p. 153
} 


\subsubsection{Newcastle Program}

At the University of Newcastle-upon-Tyne, in England, consideration of the possible uses of computer techniques in automatic or semi-automatic typesetting apparently began at least as early as 1957-1958. ${ }^{21}$ However, it was not until 1961 that Duncan and his associates at Newcastle received a grant from DSIR (Department of Scientific and Industrial Research) for the experimental investigation of computer programs for this purpose.

Using first a Ferranti Pegasus computer, the output of Newcastle programs could be "reproduced either on a proportionally spaced IBM Executive Typewriter for "economy" work or put through a five- (or eight-) to thirty-one-hole tape converter producing the thirty-one-hole tape to operate a "Monotype' caster or "Monophoto' setter." 22

Duncan and his associates have offered proof of their "pudding", "first a report to the Institute of Printing at the 1963 IPEX including composition for a double spread in Ferranti Computer World, the communication to Printing Technology being set by Newcastle programme. This was followed by the setting of 'Look No Hands!' in the Penrose annual for 1964. ..." 23

Then, in May 1964, the English Electric KDF 9 computer was installed and 8-channel input and output paper tapes were adepted. Present program objectives include the further development of practical programs for use in industry, especially for book make-up work, then the exploration of general purpose programmes for table and display composition including the interpolation of mathematical, chemical, and other special symbols, the continuation of development activities, especially in the area of keyboard design, and the making available of the experimental facilities to other laboratories and to users in the printing industry.

Duncan reports further: "Once the existing programmes have been augmented with an effective editing routine they can form the basis of a series of tests of real work, with an attempt to specify in accurate terms the precise economies of the processing employed. This may very well reveal that a different system is needed before (and perhaps after) computer processing to make sensible economies, and it will then be necessary to try and set up at least a demonstration 'new' system for evaluation purposes." 24

\subsubsection{University of Pittsburgh Program}

At the University of Pittsburgh there has been for some time considerable interest and experimentation in machine processing and machine search of natural language text. These interests have combined the endeavors of the Computer Center staff with those of subject matter specialists,

${ }^{21}$ Duncan, 1965 (b), p. 1.

22 Duncan, 1964, p. 154.

¿Duncan, 1965 (b), p. 1.

st Ibid, p. 2. especially Prof. John Horty and other members of the Health Law Center. In addition to over six million words of computer-readable text of various statutes, the Pitt Center has a cooperative arrangement with the Lancaster Press whereby, for example, computer-readable copy of the text of The Physical Review is available even before it is printed and distributed.

With the acquisition, under a National Science Foundation arrangement, of the former M.I.T. computer-Photon equipment used by Barnett and his associates, the University of Pittsburgh teams are able to explore both aspects of high-speed composition and computer combination potentialities: ". . . This system will supply information to the information specialist which he previously did not have available. . . This includes type font, type size, and page layout in addition to the text. This will be important in identifying words or phrases in italic or bold face, thus providing researchers with an additional tool to enable true information retrieval instead of simply word or document retrieval." 25

More specifically, in the developing Pitt program, many typical difficulties have been encountered and certain anticipatory solutions sought. Ohringer describes some of these as follows: "Problems which we have had to solve to be able to implement our plan included: (1) There did not exist a paper tape reader capable of reading these [typesetting] tapes into a computer. Nor could we locate a company which was interested in designing or building one for us. (2) The codes in these tapes are not directly computer compatible. (3) The meaning of the codes in the tapes vary from printer to printer and often a single printing plant will use several different codes. (4) A complete article is not punched onto a single tape but is generally divided onto several rolls. (5) The editing changes and corrections are not incorporated into the paper tapes. (6) Some special mathematical symbols are omitted from the tape completely. And (7), the character set used by the printing industry contains several thousand characters, compared to less than 50 characters readily accessible to our computer." 26

Planned solutions include provision in the computer system for input from any of the different perforated tapes now used in the publishing and printing industries. "These include tapes which are $5,6,8,15$, and 31 channels wide. Although the codes used on these different tapes are not directly computer compatible we have written the necessary decoding programs which allow the computer to accept and make sense of these different codes." $2 \pi$

The Pitt system also envisages on-line manmachine interaction using techniques of display

\footnotetext{
25 Ohringer, 1964, p. 312.

${ }^{26}$ lbid., p. 312.

${ }_{27}$ lbid., p. 312 .
} 
console and light-pen intervention, especially for the insertion of mathematical and other special symbols.

Special mathematical symbols which do not appear in the tape as it comes from the printer are added and any necessary corrections are made. This is done on the character display tube by use of the light pen which we have attached to our PDP 4 computer." 28

\subsection{Primary Publications}

As the pioneering example of a formal scientific publication typeset by computer-photocomposer combination, Bozman's work with the cooperation of the IBM Mergenthaler team will be considered first. It is noteworthy, also, in demonstrating how effectively proofreading requirements may be reduced or eliminated by the adoption of such techniques.

\subsubsection{Computer-Compiled and Computer-Generated Primary Publications}

At the National Bureau of Standards, William R. Bozman has programmed an IBM 7090 computer, using FORTRAN programming language and FAP symbolic coding subroutines, to generate magnetic tapes in Linofilm code, for the preparation of scientific tabular data. These magnetic tapes are converted to Linofilm 15-channel perforated tapes, which are in turn used to control a Linofilm photo unit.

Some of the details of the first publication are as follows: first, the values for the transition probabilities for the spectral lines of 70 chemical elements were computed by an IBM 7090 and the results were read out onto magnetic tape in binary codeddecimal (BCD) form. Next, using a program coded in the FORTRAN language, the BCD tape was converted to binary coding for the characters making up the numerical values to be included in the tables, for the control codes, and for the formatting instructions (e.g., 10 quad-left codes were used at the end of each page in order to give blank lines between pages so that they could subsequently be cut apart.

This binary coding, output to another magnetic tape, provided the precise bit (punched hole) patterns necessary to operate the experimental magnetic-tape controlled Linofilm installation at IBM's Watson Research Center, thus, "The binary magnetic tape for input to the paper tape converter was recorded in 'records' of 250 computer 'words' of 36 bits each. . . Each computer word contained two characters to be typeset, corresponding to two rows of holes on the 15-channel paper tape. . . .

"The computation of the data and preparation of the BCD tape to the binary tape in Linofilm format took twenty minutes on a 7090 computer: the

2" Ohringer, 1964. p. 313. conversion from the BCD tape to the binary tape in Linofilm format took thirteen minutes. It would have taken about 300 hours to set the table by conventional methods." 29

Bozman notes the following observed advantages:

"The proofreading and correcting of typographical errors would also have taken considerably more time, especially since the table produced was not completely proofread (after proofreading the first few pages without finding an error, a decision was made to check only the first and last line of each page)." In addition, "computer and computer-processed data can now be prepared for publication by an automatic method that retains the high typographic quality of conventional typesetting. Better page formats with less waste space result in less cost for paper, with the added benefit of a smaller size which is easier to handle." 30

". . Automatic setting of a large amount of tabular data processed by computer for commerce, finance, scientific and governmental purposes ... [involves copy which is] relatively unsophisticated, typographically speaking, but for reasons mostly associated with the precision required and the variety needed within the framework, this has been fairly expensive by composition methods apart from the inherent error rate." 31

Errors reside not only in the re-keystroking to provide reproduction copy and/or perforated tapes to control casting equipment but also in the casting equipment itself, and, in addition, human proofreading of copy before final printing is by no means infallible.

Primary publications in the sense both of technical reports and articles in journals that deal with the subject of automatic composition itself have been noted elsewhere in this report. An example of space saving and other advantages to be achieved by the introduction of these techniques to the reproduction of reports and technical papers was provided by 1963 Mergenthaler experiments, as follows:

“. . Two experiments at Mergenthaler Linotype Company. In the first, a 43-page article (standard single-column, typewritten format from an ASTIA listing) was reset and automatically. made up with the assistance of a specially programmed electronic computer. The phototypeset article occupied only 22 pages-only one-half as much as the original typewritten article. In the second, a paragraphstyle parts list, produced directly from computer print-out as eight and one-half by eleven inch, double-column, all capital, 7-point copy, was reset in a 6-point sanserif type in a 3-column format on the same size sheets. This page carried 180 percent more material than the original." 32

"Starting with a typewritten, unjustified manuscript, with illustrations, and with simple editing

\footnotetext{
${ }^{29}$ Buzman, 1963, p. 1.

${ }^{30}$ lbid. p. 4.

31 Duncan, remarks, Computer Typesetter Conference "Proceedings", 1965, p. 6.
32: Kraft, 1963, p. 275.
} 
rules, the contents of the article were fed to an IBM 1401 computer programmed to operate on the input information in accordance with predetermined format rules. The output of the computer was a magnetic tape that carried (in coded form) justified, double-column text that had been properly made up into pages, space having been assigned for the insertion of the illustrations at the most appropriate points in the text. This tape was then fed into a Linofilm Converter to produce a tape that controlled a Linofilm Photographic Unit to produce fully composed pages, phototypeset in an attractive combination of type faces." 33

In a more general sense, potential applications in the area of primary publications are demonstrated by the experience to date in both newspaper and book composition developments.

\subsubsection{Newspaper Composition}

With the minor exception of science news reporting, the problems of newspaper text, classified ad display advertising, and stock market table typesetting have little direct applicability to the problems of handling, processing, and using recorded scientific and technical information. However, it is in this area of application that the greatest progress has been made, to date, in the use of automatic and computer-controlled typesetting techniques.

Text typesetting using unjustified tape has produced gains not only in lower keyboarding cost and increased efficiency but also in column space density, hence lower page and total issue cost. Yasaki cites Los Angeles Times experience as follows: "Any newspaper with $50 \mathrm{~K}$ circulation or about 20 typesetters can justify the rental of hardware for typesetting purposes alone at $\$ 3.5 \mathrm{~K}$ per month, Booth (L. A. Times operations director) says. ( $\$ 3.5 \mathrm{~K}$ is the average rental for a 301 configuration with out the high-speed reader and punch.)" ${ }^{4}$ Similarly, "The RCA 301 can output in excess of 30,000 lines per hour producing justified tape for a 21 in. column in seventeen seconds." 35

Furthermore, "Major benefits of computer controlled typesetting systems are speed and efficiency. Fast breaking stories can be covered in earlier editions and normal typesetting loads can be handled by fewer people. Computer justified lines usually contain more type and thus the news to space ratio is improved." 36

It has been claimed that: ". . . The greatest number of automatic systems to date are in newspaper composing rooms, where raised type is generally used. In these systems, the typesetting is performed by tape-controlled Linotypes, which are controlled by electronic computers to arrange the type properly in the page make-up. Such systems permit the use of high-speed rotary presses, and thus lend themselves to the rapid, economical

\footnotetext{
$33 \mathrm{Kraft}, 1963$. p. 276.

36 Yasaki, 1963 , p. 29.

33 Duncan, 1964, p. 146.

${ }^{35}$ Goodwin, 1964, p. 34.
}

production of the large number of copies demanded. ... ." 37

As of 1964, it could be reported that "more than two dozen daily papers now own, lease, or have ordered computers." 38 More specifically, "The use of computers in printing has made greatest headway in newspaper publishing. The first computers used in production were RCA 301 models installed at the Los Angeles Times and Mirror and the West Palm Beach, Fla., Post-Times almost two years ago. Since then, 22 other computers have gone into operation or are on order by the nation's dailies, according to a recent study by the American Newspaper Publishers Association."39

In the same year, 1964, opinions of newspaper publishers with respect to the introduction of the new techniques, despite possible labor-relation difficulties, could be reported as follows: "The Toronto Telegram had 49 linecasting machines. Publisher John Bassett has been able to scrap seven, immobilize eight others, and put another 25 on part-time production. Nearly all the type for his 60- to 70-page, 200,000-circulation afternoon daily is now produced by just nine automatic, computerfed linecasters, which require only four monitors a shift to keep them running. The 200-man composing room is now about 120 , and wage savings alone are estimated at $\$ 10,000$ a week." 40

" 'If newspapers are to operate efficiently', says publisher John S. Knight, 'they must automate'. Knight's Miami Herald houses an IBM 1620 and an IBM 1460 that set type, bill advertisers, and-in the absence of a strong union - can be harnessed for other labor-saving tasks." 41

The "notable installations" remarked on by Goodwin, as of 1964, included "the Los Angeles Times, Palm Beach Post-Times, and Washington Post with RCA 301 systems, and the Daily Oklahoman Times, Washington Star, and South Bend Tribune with IBM 1620 systems." 42 More recent examples include the use of the NCR 315 in Florida, where "the people at Orlando Sentinel Star claim to be setting more editorial copy by computer, as distinct from classified ads, than any other newspaper"; and the British plans for ". . . The Evening Post-the new evening newspaper to be launched by The Thomson Organisation Limited in September . . . will be printed by the Web-offset process using a new computerised typesetting system and photofilm composition. The photographic composing technique -will be initiated by twelve keyboards coupled directly to a general purpose digital computer - the Elliott 803B." 43

Newspaper composition applications also illustrate some of the additional advantages promised by automatic composition: direct tie-ins to communication links, merging of "core" with peripheral

\footnotetext{
${ }^{37} \mathrm{Kraft}, 1963$, p. 276.

38 "Signs of the Future?", 1964, p. 88

${ }^{39}$ Blundell, $1964,28$.

40 "Signs of the Future?", 1964 , p. 88

al Ibid.

${ }^{42}$ A. H. Beaven, Comp. Type. Conf. Proc., 1965, pp. 191, 193.

${ }^{43}$ The Computer Bulletin 9, 19 (1965).
} 
or locally revised material, and semi-automatic or automatic aids to two-dimensional composition, especially for display advertising. First, internally: "The New York Times columns are compiled on hot metal linecasters from tape produced from a Friden Add-Punch which adds the current price to the preperforated tape carrying the main invariant body of the table." 44

Next, with respect to wire association services or to the various members of a particular newspaper chain where it is desired to merge national with local interest copy. Thus, the same basic copy may be processed many times "to produce columns for editions sold in ten different geographical zones. (These zone editions may differ in their local news content, but contain common state, national, and international news.)" 45

Another example is the Associated Press: "AP in New York have recently installed an IBM 1620 system which puts out six-hole TTS tape direct over the wire news network so that it is punched out at the newspaper plants ready for the line-casters. . . . "Local alterations to wire tape services can be added by a simple tape merging system while the material is held in a magnetic tape or disc file." 46

Yasaki notes, with respect to the Los Angeles Times installation that: "Unlike other computerized typesetting systems, the Times" 301 can accommodate teletypesetter tapes of wire services (Associated Press, United Press International). These tapes already contain justification codes, but for a type size at variance from that used at the Times, and only for one column width. (AP stock market tapes, however, are compatible and being used.) The 301 has been programmed to eradicate justification codes on news story tapes, to re-justify and, if necessary, to hyphenate lines." 47

Although under active investigation and in some cases experimental trial in several different organizations, the potentialities of semi-automatic and automatic aids to typographic composition have been less applied to display advertising than to textual copy. One of the reasons for this has apparently been the line-by-line rather than area or page composition approach so far practically available.

For example, Corrado has reported on different occasions as follows: "Several major computer manufacturers are trying to do computerized display advertising with the conventional phototypesetting machines. They are finding it very hard to justify the use of the computer with their existing, line-byline machines. An area-composition machine like the Linotron is much more suitable." 48

"The basic problem in doing display advertising on a phototypesetter is that existing phototypesetters set type line-by-line. This makes it hard to set copy properly on the page. It would be better if we could set copy in block form, as can be done with an area composition device like the Linotron." 49

However, IBM, RCA, Photon, and other suppliers of equipment have been working with potential customers in this area. For example: "The applications of the Perry Publications and the Los Angeles Times center around text-matter composition.... We (RCA) are getting involved in other areas, particularly with Perry Publications, where they are now setting the Palm Beach Life and parts of the All-Florida Magazine by computer, and where they are now in the advanced stages of developing of procedures for composing display ads on the Photon machine. They are also in experimental stages in the composition of pocket books. . . ." 50 ". . IBM is currently developing a system for setting hot metal display advertising type by computer." 51

Looking toward future progress, Yasaki quotes H. C. Durbin of the American Newspaper Publishers Research Institute, Inc. as prophecying, in 1963, that: "When properly programmed, stories and pictures contained in a computer memory automatically will be justified, composed, capitalized, and positioned in the desired form at extreme speeds. . . Pictures are displayed as halftones in the proper position and size, ready for production of an engraving for letterpress printing, a lithographic plate, or any other system of printing. Tear sheets, including halftone photographs, can be produced on Electrofax paper or by Xerography directly from the face of a cathode ray tube." 52

Moreover, as Spencer comments: "Today we are in the throes of another technical revolution in printing. Metal is gradually being eliminated from composition. Released from its discipline the designer is free to place his lines of type at angles, and he can curve, cut, or display lines and juxtapose one line of type closely against another or he can, if he wishes, superimpose one word upon another." 53

\subsubsection{Book Composition}

While newspaper composition has held the lime. light in terms of practical production operations involving high speed, high quality, automatic composition to date, book composition advantages are at least on the horizon. One of the major gains from semi-automatic and automatic composition techniques is, as we have seen, the relative ease of reissue with or without corrections and updating or in different sizes and type-styles. These possibilities may offer significant advantages for scientific and technical texts. As a representative of the British publishers, Hazel Sun Ltd., has pointed out: "It is our very firm opinion that a prima facie case

\footnotetext{
4t Duncan, 1964, p. 149

15 Pay, 1963, p. 330.

t6 Duncan, 1964. $p$. I 49

47 Yasaki, 1963, p. 28.

ts Corrado, 1964, p. 115.
}

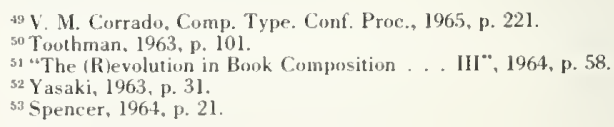


has already been made out for computer typesetting as applied to general printing." ${ }_{54}$

In 1963 it could be reported by representatives of Alden Press that "as innovators we are moving slowly into volume typesetting. We have completed many small commercial jobs and several complete books, including textbooks. We look forward to high-volume production of quality type at reduced costs on a faster production schedule." 55

In the same year, 1963, Westerham Press Ltd., in England, began a research program for computerized typesetting. At ROCAPPI (Research $O$ n Computer $A$ pplications in the Printing and Publishing Industries) book composition developments are under way on a service bureau basis. Here, "one of the first problems encountered was avoiding widows at the tops of pages. This reportedly has been solved by retaining two pages in memory storage until the first line of the third page is checked. If this line is a widow, the machine can then remake the preceding pages to eliminate it. . . . Effort has since been concentrated on programs for handling footnotes, captions and double column makeup." 56

Where the newspaper organization has control over both the format and media of input (and, as in the case of the Los Angeles Times, can impose requirements that reporters write the original version of their copy on special typing equipment), the book publisher typically does not. At Westerham Press for example, the use of a general purpose computer in automatic composition was decided upon:

"We decided on a general-purpose computer for the following reasons. We ascertained that we could accept varying types of imput; by that I mean that we would not be limited to one specific perforator. The tape had to contain certain function codes, and provided the perforators produced these function codes and produced either a five or six-channel tape we could accept them into the computer and translate them into a computer code by means of a translate table. This would enable us to accept a perforated tape from a printer or a publisher or, if necessary, from an author." 57

Blundell claims: "A special advantage of computers for book printers is that type only has to be set once. . . A copy of the book is produced by a print-out device at the same time the computer is performing its justification and hyphenation functions. Corrections in this copy are fed into the computer, which then produces the tape used by the printer." 58

\subsection{Secondary Publications, Specialized Bibliographic Aids, and Integrated Systems}

In the area of the preparation and reproduction of secondary publications, especially announcement

\footnotetext{
${ }^{54}$ Fullick, 1965, p. 23.

ss Worton, 1963. p. 99

s8 "The (R)evolution in Book Composition ... III," 1964, page 59

37 MacIntyre, Comp. Type. Conf. Proc., 1965, p. 34

${ }^{58}$ Blundell, 1964, p. 28.
}

journals, the dilemma between the high speed of computer compilation and the desire for high typographic quality of the product has been particularly acute.

On the one hand, a trend toward computer processing is developing because of such consideration as the following: "As the bulk of the text increases, it becomes increasingly necessary to seek highspeed composition and printing techniques in order to reduce the time between the editorial closing date and the actual press work.

"Response times for large cumulations will be significantly shortened through the mechanization of the prepublication manipulation activities necessary for preparation of the text.

"Although publications are now being produced by conventional techniques, the problems of updating and cumulation have made many of them too expensive to produce as frequently as required." 59

Another gain in the area of secondary publications, particularly abstract journals, announcement journals, and indexes stems from tape or computer merging capabilities. This "lies in the fact that setting takes place during the month concurrently with indexing, whereas with every other method setting commences after the month's issue has been closed and sorted." 60

On the other hand, a number of considerations with respect to secondary publications and other bibliographic tools point toward continuing requirements for good typography. First are questions of space utilization and hence of cost. In the case of many large indexes, for example, both the size of the printing run and the number of pages per volume dictate maximum page space utilization. Other reasons have generally to do with questions of user acceptance, such as the following:

"The typographical quality of bibliographical tools is an important factor, since they are intended for continual visual lookup and as such are subject to the same rigorous demands for quality and legibility as are the publications intended for retrospective search." 61

"Technical information bibliographical guides, in Great Britain at least, are themselves struggling for a wider acceptance, and I do not think that our newly converted consumers will accept standards of visual clarity lower than those we obtain with Varityper." 62

Attempts to provide the timeliness and speed of compilation and reproduction offered by KWIC (and other computer-compiled indexes such as WADEX ${ }^{63}$ ) with improvements in the typographic quality of the product range from the use of a modified 1403 chain printer to provide upper and lower case or to simulate bold face effects to full-scale computerized high speed photocomposition.

\footnotetext{
59 Sparks et al., 1964, p. 188

${ }^{60}$ Coates, 1965, p. 46

61 Sparks et al., 1964, p. 157

62 Coates, 1965, p. 47.

63 WADEX ( $W$ ord and Author Index) is a machine prepared index to Applied Me chanics Reviews, sponsored by the Air Force Office of Scientific Research and produced by the computational facilities at the University of Texas.
} 
"The results of this study indicated that the use of a chain-type mechanical computer printer would recpuire the printing of 100 percent more pages for an issue of Index Medicus than would a specially designed photographic typesetter." 64

We shall consider here as examples the Index Medicus of the National Library of Medicine, the STAR (Scientific and Technical Aerospacc Reports) of the National Aeronautics and Space Administration and the proposals for more complete computerization at the Clearinghouse for Federal Scientific and Technical Information and at the National Library of Agriculture.

The most complex and the most successful largescale computer-photocomposing installation to go into production to date is the MEDLARS (Medical $L$ iterature Analysis and Retrieval $S y s t e m$ ) at the National Library of Medicine. Production of the Index Medicus by means of the fastest computerdriven photocomposer yet in operation began in August 1964. This first issue (see also Figure 3) was comprised of some 600 pages, containing over 9,000,000 characters. and was processed in approximately 16 hours, including set-up, computer run, and correction time. It is not surprising that some have hailed this achievement as "the great bibliographic break-through of our generation". 65

It is especially to be noted that, because of the multi-purpose usage aspects of the overall system design, the question of how much depth-indexing information was to be included in each of several outputs was solved by a "compromise between the conflicting requirements of the publication and retrieval systems... (Previously) the Index Medicus subject section included an average of about two entries per article. For the retrieval system, this level appears to be entirely inadequate and the goal has been raised to an average of eight to ten subject tags per item. . . The publication average was upped 50 per cent ... and the remainder of the selected headings . . . are retained in the computer system for search purposes." 66

The Library of the Office of the Surgeon General, U.S. Army (later, the Army Medical Library, and now, the National Library of Medicine) has been for approximately a hundred years an innovator and advocate of machinable techniques. First, it was John Shaw Billings, appointed as a consultant to this Library in 1865, who collaborated with Herman Hollerith in the original ideas for mechanical processing of information punched into perforated, machine-processable cards and to whom "will always belong credit for having created the most monumental medical bibliography the world has known." 67

Developments since Billings' day have exemplified considerable continuing receptivity to new and improved techniques, specifically including con-

\footnotetext{
BA "The Medlars Story," 1963, p. 54 .

s Taine, 1964, p. 125, remarks of R. T. Esterquest.

Taine, 1964, pp. 123-124.

${ }^{67}$ Rogers and Adams, 1950, p. 271.
}

sideration given to varied possibilities for the mechanization of bibliographic tools for the processing and use of the medical literature. In 1949, "a Committee of Consultants for the Study of Indexes to Medical Literature Published by the Army Medical Library ... was appointed. . . As a result of the committee's activities, certain decisions wcre made in 1950 that radically affected the Library's publications program. The venerable Index-Catalogue, which had been published for three quarters of a century by the traditional letterpress method, was suspended. In its place were issued two separate publications, the annual Printed Catalog and the monthly Current List of Medical Literature, both prepared by the then novel method of shingling individual $3^{\prime \prime} \times 5^{\prime \prime}$ slips or cards into columnar or page formats." 68

Roughly concurrently (that is, in the period 1949 1953), a special project at the Welch Medical Library, Johns Hopkins University, began the exploration of machine methods potentially useful for bibliographic work.

". . a research contract was granted to Johns Hopkins University in October, 1948, by the Medical Research and Development Board, Office of the Surgeon General". ${ }^{69}$ This Army Medical Library Research Project had among its contract objectives that of exploring "existing and projected methods, emphasizing machine methods, applicable to such pilot projects as may be necessary."70 The work at the Welch Library continued for several years, the final report being issued in 1955. Beginning in 1951, the project maintained in punched card form the Current List of Medical Literature. Garfield has stated that this work "clearly demonstrated the ease of converting alphabetic subject heading lists to categorized or classified lists of terms." 71 Ingenious use was made of a modified IBM 101 punched card machine for the preparation of printed subject indexes.

Then, from about 1950 to 1959, "the Current List of Medical Literature . . . was typed on individual $3 \times 5$ cards which were laid out in shingle form by hand for photo-offset publication. In January $1960, \ldots$. Index Medicus was launched as the successor. . . . The entries . . . were typed with the paper tape-typewriter onto Hollerith cards and fed through the Listomatic camera, for photo-offset publication .... (they) have now established the requirement ... to publish Index Medicus with the aid of a computer. . . ."72

Next, the Council on Library Resources made a grant to the NLM for an "index mechanization project" between July 1958 and June 1960. As a result of this project, the production of the Index Medicus since January 1960 used the Listomatic Camera, IBM card punching and handling equipment, and the Friden Justowriter (tape-typewriter with a justi- 
fied right hand margin). Bibliographic citations, author entries, and subject headings were punched onto paper tape by Justowriters and proofread, the tape pernitting automatic duplication of entries on IBM cards in different formats. Collating and sorting equipment produced a mechanically sequenced deck of cards. Proofreading was from a positive paper print produced by the camera. Following correction the film was processed, and columns stripped up for page makeup. The monthly publications were produced by photo-offset. The 12 monthly decks of cards were interfiled by IBM equipment to produce the annual Cumulated Index Medicus of the American Medical Association.

In August 1961 the NLM undertook its MEDLARS project (Medical Literature Analysis and Retrieval System) by contract with the Information Systems Operation Division of the General Electric Co.'s Defense Systems Department. The monthly Index Medicus, the annual Cumulated Index Medicus, the annual printings of Medical Subject Headings, the Bibliography of Medical Reviews, and recurring bibliographies all continued. Under subcontract let to Photon, the Graphic Arts Composing Equipment (GRACE) was to be designed to compose finished copy on film suitable for offset printing. Most demand (nonrecurring) bibliographies and all statistical information to be prepared by the system would be printed by a standard computer printer, in single font copy on continuous paper or on $3 \times 5^{\prime \prime}$ card forms. ${ }^{73}$

The equipment now used in MEDLARS is the developmental prototype of what is now commercially offered, for delivery in the near future, as the Photon ZIP. It is noted that: "GRACE accepts electrical signals from a magnetic-tape transport operating fully under her control. These signals arrive in bursts of coded characters which represent a full line of legible type across the multi-columnar page. This type is made up of a combination of 226 different alphanumeric symbols which are set on film or paper at a rate in excess of 440 characters per second." 74

Operations since August 1964, when GRACE produced its first Index Medicus issue are typically as follows: Abstracts are punched on Flexowriter perforated tape, and these are read via a high speed photoelectric tape reader into a Honeywell $800 \mathrm{com}$ puter having 65,000 words of magnetic core memory. The 10,000 to 15,000 citations per issue are arranged for multiple entry and cross indexing and stored on magnetic tape. Then the computer generates an output tape used to drive a Photon 900-type photocomposer, which composes at a speed of 300 to 500 characters per second, setting the entire Index of nearly $2,000,000$ words in about 16 hours.

It is estimated that with this new system "the monthly issue of Index Medicus will require but five days for completion of the film 'manuscript',

\footnotetext{
${ }^{73}$ National Library of Medicine, 1963 (311), pp. 4-5; Taine, 1964, pp. 119-124

${ }^{74}$ Taine, 1964, p. 122.
}

and recurring bibliographies will be processed in one or two days." 75

The format of the Index Medicus is based on the unit citation. ..."A typical unit entry consists of the following elements: the names of all authors cited in the original publication, the title of the article either in the original English or in English translation, the abbreviated journal title, the volume number, inclusive pagination, date of issue, and a symbol indicating the language of the article if other than English." 76

The MEDLARS experience is important to those concerned with improved handling of scientific and technical information in several ways over and above the high-speed, high-quality secondary publication aspects. First, it is a system involving multiple computer processing and outputs and multiple types of usage of the output material. Record tapes generated in computer processing and containing not only the unit citation data but search-selection tags with respect to subject content, geographical location, type of document, and other tags as well are retained in the master file while copies are also circulated to cooperating organizations elsewhere in the country.

Secondly, the MEDLARS system plan involves consideration of more than one type of usage and more than one type of user requirements. For example, "two basic output techniques [are] available in MEDLARS. For routine man-machine communication, some of the recurring bibliographies and most of the demand searches, a high-speed computer printer will provide single font copy in continuous paper, or on $3^{\prime \prime} \times 5^{\prime \prime}$ card forms. The Index Medicus, Cumulated Index Medicus, Bibliography of Medical Reviews, most of the recurring bibliographies and a small number of demand searches ... [will be produced by] GRACE. . .."77

Finally, the MEDLARS experience has a bearing on size of character repertoires required and minimization of page costs for massive secondary publications of this type. "We need enough fonts to distinguish the subject headings from the citations and to distinguish, within the citations, the beginning of the journal title abbreviation from the author and title. We have a 6-point font, which is a quite small size, probably the limit of what you would want, for the citation itself. We are trying to pack 10,000 characters on a page. Our present load is 5 to 6 million characters a month and will soon be 10 million characters. It costs a lot of money to print that many characters; therefore, we have adopted the smallest type size that we think is at all reasonable." 78

Another example of a secondary publication based on photocomposing techniques is the National Aeronautics and Space Administration's STAR Scientific and Technical Aerospace Reports). In the early 1960's, Information Dynamics Corporation

\footnotetext{
${ }^{75}$ Ibid., p. 123.

${ }^{76}$ Ibid.. p. 112.

${ }^{7 \pi}$ F. B. Rogers, discussion, Sparks et al., 1964, p. 194.
} 
developed for the Scientific and Technical Information Facility (operated by Documentation, Inc., for NASA) a tape-operated system for photocomposing STAR. Although eventually more comprehensive computerization of these operations is planned, time pressures for system installation in advance of some of the more recent technological developments both in computer procrams and in photocomposer equipment dictated the use of a modified Friden $\mathrm{LCC}-\mathrm{S}$ keyboard to produce a paper tape to drive a Photon machine.

Price reports: "Due to time limitations, it was decided to begin operation from the Photon keyboard (on-line) and then at a later date, increase production capacity by driving the Photon with punched tape generated from four off-line keyboards... The Photon was delivered in April, 1962 , and within the month, journal copy was being produced". 79

An illustration of some of the difficulties involved in incompatibility between machine languages is also afforded by the NASA example. The Photon equipment to be used then required a special code and tape format, incompatible with IBM $1401 \mathrm{com}$ puter requirements necessary to process the information used in STAR for storage and retrieval purposes elsewhere in the system. The decision was: "to stay with this unique code rather than use standard eight-channel coding which would have required the design of a special translator for the Photon. For computer input, commercially available equipment would be used to translate the Photon code to IBM 1401 language as an integral part of the tape-to-card conversion." 80

"Searching capability should be derived as an intrinsic by-product of the primary system complex which, in the case of the NASA system, is heavily oriented to scheduled publications. It is necessary to achieve this capability at the lowest possible cost in terms of additional operating dollars and design effort over the basic needs of the system" .81

The Clearinghouse for Federal Scientific and Technical Information (an outgrowth of the former Office of Technical Services) is, like NASA, presently using an interim approach whereby its U.S. Government Research and Development Reports is paper tape produced and transmitted to the U.S. Government Printing Office for composition on Linofilm. System planning for a computer system is, however, well under way.

Similarly, at the Department of Agriculture a comprehensive proposed system, ABLE (Agricultural and Biological Literature Exploitation) would involve computerization of the preparation of the Bibliography of Agriculture. "In our proposed system, the B of A would be composed and printed from a magnetic tape using either a printer with upper and lower case letters or a photocomposition machine.

\footnotetext{
т9 Price, 1963, p. 261

*n Ibid. p. 261 .

Swid. 1964, p. 322
}

"Further computer processing would compose the columns for a page and the entire page would be printed at one time, producing camera copy with no stripping or other hand processing". 82

In each of these applications, systems planning calls for an integrated operation where the information required for announcement or indexing can be automatically merged with files permitting mechanized search, selection, and retrieval. Additional comments on this point include the following:

"Since citations for all announced documents would be composed on Justowriters, the tape used for input to the Photon could also be used as input to the computer search and retrieval system, thereby eliminating repetitive keyboarding of information." 83

"It seems clear from our preliminary analysis that the tapes generated in the program we are proposing may prove to have an importance equal to or greater than their use in the graphic reproduction of scientific documents. Once original papers are available in some form of punched or magnetic tape, then all of the potential of computers is opened to development and use". 84

"...We are looking ahead to the use of computers, both to speed the process of setting structures and to investigate further the usefulness of the output of such a system in the searching and retrieval of chemical structures." 85

An approach combining the ideas of using computer-usable material for multiple purposes and of converting text into machine language form at the earliest possible stages of preparation for publication has been advocated by research workers at ITEK Corporation and more recently at Inforonics.

First, the ITEK Crossfiler is a special purpose device which was developed for the Air Force Cambridge Research Laboratories. It has been designed "to manipulate a machine-interpretable paper-tape record of a Library of Congress catalog card and to produce sets of diversely headed cata$\log$ cards automatically". ${ }^{86}$ Lipetz describes techniques and equipment for simultaneous production of manual and machine-usable book cataloging records as a first step in introducing a machine record system for the book catalog at the Research Library of the Air Force Cambridge Research Laboratories, Hanscom Field, Bedford, Mass. Punched paper tape was created in the course of continued production of $3 \times 5$ inch index cards as similar as possible to those in the existing catalog. The input typing format, designed for a special-purpose computing machine, the Crossfiler, produced sets of catalog cards at less cost than the utilization of a general-purpose computer. Sets of catalog cards were obtained with identical text in the body of card but different tracings (entries at the top of card for title, abridged title, subject, etc.) derived

\footnotetext{
K2 Department of Agriculture, "Report of Task Force ABLE," 1965, p. 4

8.: Price, 1963, p. 261.

Kuney, 1963, p. 24\%

Hs Kuney and Lazorchak. 1964, p. 305.

wharks et al., 1964, p. 170.
} 
automatically from the text contained in the primary punched tape. ${ }^{87}$

Next, a grant from the Council on Library Resources enabled Inforonics to explore, in cooperation with the Library of Congress, the possibilities for multi-purpose recording of bibliographic information in computer-usable form. "The firm [Inforonics] . . . has undertaken to demonstrate that bibliographic information, when suitably punched into a perforated paper tape record by a tapeproducing typewriter, can be reproduced in any form, whether complete or abridged, typewritten or printed in any one of a variety of type faces, as may be required for the various records used in library operations." 88

Pertinent illustrative details of more recent Inforonics developments excerpted from a recent Buckland report are as follows:

"The approach used in this project is to develop a system which identifies the text item content in enough detail to carry out any functional use of that item. The identification does not need to represent the typographic form of the end use, because most aspects of the output form, such as the typeset journal itself, can be machine derived if the identity of the item is known. . . .

"The typographic control word specifies the output form of an information item. The specifications contained are:

Font

Point size

Column width

Quadding (flush left, flush right, center, justify, center justify)

Leading

Set size

Special commands. ...

"A record from a relatively simple input typing operation can be processed to form a multiplicity of high typographic quality, complex output products. Since the scheme developed was output independent, any typesetting machine can be controlled from the input record and the keyboard operator's training is not difficult. . . .

"This recording procedure is different from other text recording approaches, because it sucutifies the journal text item content rather than its typographic form. This procedure allows the primary record to be processed into a variety of secondary products, regardless of their typographic forms. A computer processing system has been developed to produce the primary journal typesetting tape, an author index typesetting tape, and an abstract journal entry typesetting tape. All of these programs operate from a single input manuscript record. . . .

"The processing of the primary record could be extended to create citation index entries, subject index entries, permuted title index entries, ordinary

${ }^{47}$ Lipetz, 1963, pp. 119-124.

*s Council on Library Resources, 1964, p. 1. title index entries, and perhaps an abbreviated announcement publication." 89

Buckland concludes: "The accomplishments so far have demonstrated that it is possible to satisfy both the primary journal publication process and the requirement of data recording for certain secondary uses with a single publishing system." 90

\subsection{Service Centers}

Closely related to the newly emerging applications of book composition is the notion of the service bureau or center which will do automatic typographic quality composition on input from a variety of sources. Already, "service center developmental activities are fermenting and materializing in many sections of the country". ${ }^{91}$ Examples include ROCAPPI (Research $O$ n Computer $A$ pplications in the Printing and Publishing Industries) both in cooperation with other Philadelphia firms (W. B. Saunders and Computer Composition, Inc.) and in England (Hazell Sun Ltd.), Keydata Corporation (Boston), and others.

Developments involving linkages with communication networks will probably encourage the growth of these and other centers. Duncan reported at the 1964 Computer Typesetting Conference that". . . data relay equipment over wired networks . . . is at last available ... of adequate performance and at a reasonably economic cost. We can now expect to see the expansion of service bureaux activities and they may eventually be able to provide computer typesetting programmes for their customers and an off-peak service at acceptable rates." 92

As early as 1963 Morton reported that, at Alden Press, various publishers were exploring the feasibility of "furnishing punched tape for us to process through the computer. This possibility is recognized, as is the possibility of Data-Phone transmission from publisher to typesetter where volume or service demands so indicate." 93

Still another potential advantage of computerized systems is that: "Output printing of auxiliary data stores using high-speed typesetting techniques will be possible and, in the case of national libraries, highly desirable. The attractive feature of this is, of course, the assurance of frequently up dated editions of the class schedules and subject heading list." 94

In this connection, it is of interest to note that proposals for using computer printouts to produce updated and revised UDC (Universal Decimal Classification) schedules have already been made by the United States National Committee for the International Federation of Documentation. ${ }^{95}$

The most extensive service bureau experience to date is undoubtedly that of ROCAPPI. They

\footnotetext{
${ }^{89}$ Buckland, 1965, pp. 2-20.

919 Ibid., p. 3

9 "Computerized Typesetting", 1964, p. 6.

${ }^{92}$ Duncan, Comp. Type. Conf. Proc., 1965, p. 5.

${ }_{93}$ Morton, 1963, p. 100.

${ }_{93}^{93}$ Morton, 1963, p. 100.
${ }_{94}$ Sparks et al., 1964, p. 158.

${ }_{95}$ Mills, 1964, p. 71.
} 
accept from various customers manuscript copy prepared on Justowriter, Dura, Fairchild TTS and other input tapes and formats. This is processed through an RCA 301 computer to prepare justified, merged, and corrected copy for use in typesetting operations. With respect to output they report: "There is a separate programme for each output device, but they all produce the standard RCA 7level paper tape. Obviously, it is nccessary to convert for Linofilm, Lumitype, Monophoto and the new ATF B8 machine. We have at present output programmes for the Linofilm, for the ATF, both $B$ and B8 models, and for TTS linecasting equipment. We are working as well on output programmes for the Monotype and Monophoto and for the range of Photon-Lumitype equipment. . .

"Output is of course coded according to the converter concerned. Thus for the Linofilm our output is coded for a converter produced by the Navigation Computer Corporation in the States. Since the Linofilm tape has 15 channels, we have to produce three rows of five channels per character. The sixth channel is a synchronization hole perforated on the first row at every three-row character, and used by the converter to synchronize the input tape with the output tape. The seventh channel is parity, which is ignored by the converter." 96

A recent example of ROCAPPI efforts is the computerized typesetting of Nuclear Applications, a bimonthly periodical published by the American Nuclear Society.

A special case of service center operation involving dissemination of news and current trade development information to its subscribers is Composition Information Services, Los Angeles, which issues at regular intervals the CIS Newsletter. In the August 1965 issue, for example, coverage was provided to the impact of potential automation and implied re-education as viewed by the 46 th International Association of Printing House Craftsmen, news of a grant for continuation of Newcastle projects for computerized typesetting, and reporting of phototypesetting equipment at an international exhibition in Paris, including Hell, K. S. Paul (P\&M) Filmsetter, CAE, Monotype, Photon, Mergenthaler, and others.

\section{Problem Areas}

Notwithstanding the rapid progress that has been made, in the past two years in particular, toward the use of high speed composition techniques that preserve relatively high typographic quality in the printed output, a number of problem areas remain. The first of these is that of adequate character sets, especially since the new high speed composition techniques (whether linked to computers or not) have in many cases restricted available character repertoires and have in addition raised new problems of encoding and alphabetizing. Another

s. T. H. V. Archer, Comp. Type, Conf. Proc, 1965, pp, 120-121. problem area is that of programming and programming languages.

Questions of new impacts on management and systems planning include problems of changes in job production concepts, in planning for multiple off-line processing, in resolving the problems of requirements for proof copy, and in adjusting to the differences to be faced as automation tends to replace traditional industry practices. Closely related are the questions of human factors and system design, including such specific questions as the re-design of keyboards and the possible roles of automatic character recognition techniques and communication networks in new, integrated systems.

\subsection{Character Sets, Encoding, and Alphabetization}

One of the first and most obvious problems in high-speed photocomposition and/or computercontrolled publication, is that of adequate character sets, especially for scientific and technical information texts. For example, "a large number of symbols is needed to set mathematical equations more than is available on many composing machines." " Another obvious requirement is for diversified character sets, especially in the sense of providing the same characters in boldface, italic, and other styles.

With respect to the latter requirement, some representative comments to be found in the literature are as follows:

"Special attention must be given to the library practice of using changes in type faces or of type fonts to convey certain meanings. . . Mechanization of typesetting for such publications must take these semantic uses of typography into consideration. . . Careful attention must be paid to providing those visual cues which can assist this searching activity. Appropriate use of type size, position, boldface, italics, underscoring, and white space can achieve this result."'2

"The very complexity of concepts in a technical index makes the use of bold type essential for marking a clearway through the apparent maze of indexed material." ${ }_{3}$

". . When you mass bibliographical citations on a page and reduce their size, you place demands on the human eye which must be taken care of by providing a variety of type sizes and a flexibility in placing the images of the characters in the proper place." 4

Traditionally, of course, these requirements have been met by relatively unlimited repertoires in conventional printing equipment. Moreover, type faces were often designed to reflect niceties of both pleasing appearance and space utilization. With the introduction of phototypesetters com-

\footnotetext{
Kraft, 1963, p. 8.

Sparks et al., 1964. p. 161

Coates, 1965, p. 47.

'D. E. Sparks, discussion, Sparks et al, 1964, p. 195.
} 
promises at least as to aesthetic quality of individual characters have often been required. ". . . The concession which printers and their customers had, in effect, made in accepting photosetting ... meant that they had accepted that the type character would have the same proportions whatever size it was reduced to or enlarged to by optical means, rather than each size having its own proportions based entirely on aesthetic considerations. This was a step on the path to the quantitative analysis of typographical work." ${ }^{5}$

.While both the Linofilm and Photon machines have quite satisfactorily large character sets $(1,400-$ $1,500)$, and considerable coverage can be built up with specially designed grids or discs, grid and disc changes substantially reduce effective rate of throughput and/or require multiple passes.

Problems of intra-system compatibility may also arise. For example, with respect to NASA's Photon installation for the production of STAR, ". . . Specifications were prepared for Photon type fonts to be used in conjunction with LCC-S (Line Casting Control-Special) Justowriters. Each font or face had to be compatible in relative width value not only with Justowriter type but with each of the other fonts used on the Photon disc. This latter limitation arose from the fact that although font selection on the disc can be controlled automatically from tape, selection of the corresponding style cards, which provide the escapement or width value for characters in each face cannot be controlled automatically. Therefore, several faces had to be based on a single but common set of escapenent values. . . "The Univers family of type was found to provide the compatibility sought. Medium, bold, and Italic Univers faces could be composed using the single set of escapement values for bold face characters. The Greek alphabet in upper and lower case as well as special mathematical, chemical, and logic symbols were arranged on the disc so that they could be composed and set on the same Univers Bold escapement values." 6

The introduction of electronic character generation techniques as integral parts of high speed composition systems again seriously restricts the size of character sets available on any one pass or run and aggravates the character sub-set switching problems.

The use of computers for output of material to be reproduced and distributed, however, has until very recently imposed either a radical departure from previous standards of acceptability (restriction to a limited, upper- or lower-case only set of no more than 64 characters and symbols) or the painful, costly, and time-consuming alternative of resetting the computer-printer output in accordance with traditional methods and with the all too probable likelihood of introducing an unacceptable incidence of errors.

\footnotetext{
s Fullick, 1965, p. 24

${ }^{6}$ Price. 1963, p. 261
}

Duncan comments on the last point as follows: "Computer users, who have devoted considerable effort and money to produce relatively error-free results, are not likely to tolerate gratuitous errors introduced by other operators using unrelated technologies." 7

In most cases, the common complaint is that "[The upper-case limitation] reduces readability so much that the page layout must be extremely open if the material is to be usable at all. With typeset matter, a much more compact page layout can be prescribed, in fact, the readability of typeset copy can be maintained even if smaller type is used, which produces an even higher degree of compactness." 8

The so-called "upper-case limitation" in the production of bibliographic tools has apparently been a major deterrent to more widespread adoption of automatic data processing capabilities. Clapp, for example, comments as follows:

"Until there is a decent font available, it doesn't pay anyone to put a great deal of bibliographical material into machine-readable form. Until there are large quantities of bibliographical material available in machine-readable form, it doesn't pay many libraries to engage in machine processing." 9

The same commentator has remarked elsewhere: "As long as Mr. Watson would only give us a character font in capital letters there could not be any great fervor to load [machines with bibliographical information records]. If I may say so, the whole picture of automation in libraries from the thirties right down to the present date has been controlled by that uppercase limitation." 10

In a certain sense, a modest degree of typographic quality can be achieved directly from high speed computer-driven line printers, but with necessary compromises in the output speed to improve the appearance of the product. For example, Johnston reports: "We do in fact get a reproduction of bold typeface. The place names are overprinted four times, using what is called a space suppression feature on the machine." 11

The IBM 1403 chain printer involves use of a continuously moving chain consisting of 240 character positions usually divided into 5 sets of 48 characters each, so as to minimize access time to the appropriate characters to be selected. However, by redividing, a character set of 120 can be accommodated, although at significant loss (up to 50 percent- 60 percent) of printer speed. This technique is used, for example, by the Chemical Abstracts Service to provide upper and lower case for the KWIC index, Chemical Biological Activities (Figure 49). In addition, the cover of the Proceedings of the Literary Data Processing Conference [Bessinger et al., 1964] illustrates an experimental concordance of the Gothic Bible which "employs

\footnotetext{
${ }^{7}$ C. J. Duncan, remarks, "Computer Typesetting Conference Proceedings," 1965 p. 3 .

${ }^{3}$ Kraft, 1963, p. 275

${ }^{9}$ V. W. Clapp, discussion, Sparks et al., 1964, p. 194.

${ }_{10}^{10}$ Clapp, 1964, p. 54-55.
} 
carbox omlde and S-exo- ethyl-2- nor bornene-s-endo- corbox amlde moderately heve carbox amlde and s-endo- ethyl-2- nor boriene-s-exo carbox amlde. more effective th FROM 3- ETHYL-4- METHYL- ANO 4-ETHYL-3- METHYL- PYRIOINES.

6.7- BENZD MORPHANS FROM 3- ETHYL-4- METHYL- ANO 4-ETHYL-3- METHYL- PYR1O1NES.

N.N-dl ethyl-S-endo- methyl-2-nor bornene-s-exo- carbox amlde has

one and $178-N, N-d l$ methyl-17- ethynyl andro st-s-en-3- ol do not have androgenlc actlon Inhlblt growth of green color In Euglenagracilis

Inhlbit growth of green color In Euglena gracilis

formation of green colonies of Euglena graclils [effect remalns after removalof agents] CHLOROPLASTS OF EUGLENA GRACILIS AFFECTEO BY FURADANTIN.

moderately Inhlbit growth of of Euglena gracilis and Inhibit formation of green colonies o AS BLEACHING FACTDRS FDR EUGLENA-GRACILIS.

Il gonadotropin increases excretion of estrone estradiol estriol and total neutra

di acetate strongly increases excretion of tri methyl amine and urea In perch IPerca-f STIMULATING HORMONE ON THE EXCRETIDN DF TRI METHYL AMINE ANO UREA IN THE PERCH (PERCA tetracycline antlblotic increase excretion of urea ond sodium In urlne of humen with renol ALLOXAN ON THE AORENAL CORTICAL EXCRETIDN. A COMPARATIVE STUOY IN MALE. FEMALE ANO PREGNAN action of r-cells; blocks excretory action of r-cells of anterlor pltultery of mele EXDPHTHALMDS-PRDOUCING ACTIVITY IN THE SERUM AND IN THE PI CLINICAL ANO EXPERIMENTAL PHARMACOLOGY OF PARENTERAL SPIRAMYCIN.

nicotine response was restored by exposure of tIssues to noradrenaline [thyroldectomy reduce BY OE DXY RIBD NUCLEIC ACIO EXTRACTS OF THE GROWTH INHIBITDRY EFFECTS DF B- AZA GUANIN CHLORPROMAZINE FACILATION JF HEPATDCARCINOGENESIS IN THE FEMALE ISTAR RA ANTIBIOTICS AS BLEACHING FACTDRS FDR EUGLENA-GRACILIS. NIL NOCERE. RENAL FAILURE AFTER GENERAL ANESTHESIA WITH ESTIL.

and amplitude of contraction In fallure of isolated heart [perfused] caused by pentobarbi STRESS IN THE BIPHASIC CHANGES IN FASCICULAR NUCLEDLAR SIZE IN THE RAT ADRENAL:

lncreases stze of nucleoll in fasclcular zone of adrenal glands of rat; hypertrophy is b of nlctating membrane and causes fasclculation [These results show that the nicotinlc and vomlting. vertigo. vasospasm. feeling of coolness and strange taste and does not alter b A CDMPARATIVE STUDY IN MALE. FEMALE ANO PREGNANT RATS.

and causes protelnurla In female human during gynecologlcal surgery glven Intravenou sterone in blood of male and female non-pregnant rat but does not increase content of

sterone in blood of male and female non-pregnant rat but moderetely decreases content o has hyperglycemlc action on female pregnant and non-pregnant and on mele retiaction of of cortlco sterone in blood of female pregnant rat

of corticosterone in blood of female pregnant rat

saluretic action on saline-loaded female rat

carclnogenlc action on Liver of female rat. action increased by chlorpromazlne

OF HEPATOCARCINOGENESIS IN THE FEMALE WISTAR RAT BY 2-ACET AMINO FLUDRENE.

so that before spawning fish is driven towards fresher water by need for osmotlc b fludro cortlsone acetate hormone strongly inhiblts growth 2-acet am Ino fluorene weakly has carclnogenle action on liver of femele FEMALE WISTAR RAT BY 2-ACET AMIND FLUDRENE.

blocked by pretreatment with $9 \alpha-$ fluoro cortlsol

$9 \alpha-$ fluoro cortlsol hormone strongly decreases content of cor alkyl fluoro phenyl carbinols tested for choleretlc actlon [Lowe ALKYL FLUORD PHENYL CARBINOLS WITH CHOLERETIC ACTIVITY.

5- fluoro uracli has toxic action on Escherlchla coll whileo INOUCEO $1 \mathrm{~N}$ E. CDLI K-12 BY 5- FLUORO URACIL.

[as HCl] and pyridoxal iand follc acld

L- lysine leucline histldine follc acld pyrldoxine [as HCl] ond pyrldoxel iend follc THE ACTIDN DF FOLLICLE STIMULATING HDRMDNE DN THE EXCRETIDN DF TRI METHY ACTION DF CHLDR TETRACYCLINE AS A FOOOSTUFF ADOITIVE ON GLYCOLYSIS IN PIG MUSCLE.

thyroxine moderately inhlbits formation of caudal part of Muelleris duct of chlcken embr

formation of strongly inhlblt formation of caudal part of Mueller's duct of chlcken embr hormone does not inhtbit formation of cortlco sterolds in human with non endocrlne

testo sterone hormone increases formation of fructose in accessory organs of castrated bu of Euglena graclils and Inhlblt formation of green colonles of Euglena graclils leffect re estradiol Inhiblts formation of Mueller's duct of chlcken embryo

pressure of mediumi stimulates formation of osmotically sensitive spheroplasts durlng cel wth thyroxine strongly inhlblt formation of strongly Inhlblt formation of caudel pert of growth of and cause atyplcal cell forms in Baclilus megaterium

with the clsold and transold forms of the molecule]

plmelic acld in acld-soluble fraction of Escherichla coll

In lsolated mitochondrial fraction of llver of rat and in lsolated liver of rat

spawning fish is driven towards fresher water by need for osmotlc balance sodium in rabblt and frog

hormone increases formation of fructose in accessory organs of castrated bull, ram. boar, ANO RELATION TD SEMINAL FRUCTOSE.

and does not alter electrical function of heart in human with advanced neoplastic diseas DF EUGLENA GRACILIS AFFECTED BY FURAOANTIN.

nitrofurantoln[furadantin] and nitrofurazone antiblotic moderetely lnhl

DF NITRO FURANS. I. AMINO FURANS

2-substituted S- nitro furans do not Inhlbit growth of staphylococcus aureus, Esc REOUCTION OF NITRD FURANS. 1. AMIND FURANS

drugs, action not altered by ganglionlc blocking agents, mono amine oxidase Inhibltors NIL NOCERE. RENAL FAILURE AFTER GENERAL ANESTHESIA ITH ESTIL.

estll general anesthet lc decreases urinary output and Increases

not cause involution of thymus gland in male rat

and cause involution of thymus gland in male rat given subcutaneously

increases pressure in mamary gland of rabblt; weakly has hypertensive action on rat: we

of catechol amlnes in adrenal glands

Figure 49. Sample page, Chemical-Biological Activities. 
the upper-and lower-case print chain, and . . . a new technique of printing boldface by overstriking the headword in each citation. The Gothic character 'thorn' was synthesized by striking ' $p$ ' over 'b'." 12

The question of available character sets affects also the initial keyboarding operations for input to further machine processing. The shift key on an ordinary typewriter provides a total character set availability that is actually double (or nearly so) the number of keys actually operated by the typist. Where perforated tape is mechanically produced as a by-product of such keystroking, it is usually the case that a non-printing symbol or "precedence code" is punched into the tape as a result of the depression of the shift key. Extensions of this principle (e.g., red- to black-ribbon shifts) multiply the available character set size even though the differentiations may not appear on the hard copy.

Similar considerations apply to the keypunching of standard punched cards by the reservation of certain punch combinations as control and precedence codes. Examples of elaborate code conventions adopted for keypunching of text include reports by Nugent (1959) and by Ray (1962), both of which point out some of the requirements and some of the difficulties encountered with respect to providing a manual for the keypunching of natural language texts. An even more sophisticated encoding-transliteration scheme is detailed by Newman, Knowlton and Swanson (1960) as necessary for the keypunching of patent disclosures in which a very wide variety of boldface, normal, italic faces and of special symbol insertions must be provided for.

Flexible selection from amongst a large variety of available characters and symbols may thus be provided for, but at the high expense of multiple initial keystroking, multiple code-sequence processing and redundant storage requirements for each "actual" character eventually to be reproduced. For example, with respect to the early Barnett experiments using Photon, it is claimed that the proportion of control and precedence coding to actual character-selection identifications could run as high as 75 percent of the total input. ${ }^{13}$ While possibilities for re-design of keyboards and for multipleoutput from a single key depression may alleviate this problem somewhat, ${ }^{14}$ the difficulties of precedence coding to achieve larger and more versatile character sets remain a major problem area.

In this problem area of character sets required. as in most others, a critical difficulty of course is that of defining the problem itself. What are the requirements, both as to coverage and available set size, for various applications, especially including primary and secondary publications involving scientific and technical information?

A special case of precedence coding is the escape character suggested for use in adapting standard

\footnotetext{
${ }^{12}$ Bessinger et al., 1964, Title page.

13"The (R)evolution in Book Composition . . . IV, 1964, p. 68

is See also p. of this report.
}

codes such as ASCII or the British Standards Institute/International Standards Organization 6and 7-bit codes to local variations and substitutions such that larger character repertoires may be accommodated. Chadwick comments:

"This 'Escape' is a vital and very interesting character in this set. .. You use the normal code up to the point where you really require to introduce your own special characters - you encode 'Escape' and from then on you can use your private characters. It is a character which allows you to say you are using the standard code when in fact (maybe only temporarily) you really are not." 15

A few illustrative examples expressed in the literature with respect to this question have been cited in an earlier section dealing with two-dimen. sional composition capabilities of some of the new techniques. More generally, such estimates as the following have been made:

". . Publication of the generally disseminated library products and of the frequently cumulated publications can probably be accomplished with a character set of 300 elements. . . . Larger, more comprehensive, and more carefully edited publications whose content requires representation of a wider range of language and composition elements will probably require character sets of 500 characters or more." 16

"To get an indication of the number of different alphabetical characters that may be used in a single scholarly book, I asked Mr. Theodore Besterman to count the different pieces of type used in his World Bibliography of Bibliographies. This he did, and as shown in the preface to the third edition of this work, he used approximately 1950 different pieces of monotype . . . There are a number of alphabets that are not included in Besterman, as well as mathematical symbols, etc." 17

Added to the number of different symbols, in bibliographic aids generally the use of type style and weight changes are specifically used to "cue" the scanner or searcher to particular types of information presented. For catalog cards alone, there are severe problems, in terms of machine processing, of mixed font, mixed weight and point size, mixed alphabets and formats. In addition, varying conventions of abbreviation, punctuation, and other types of specialized representation have been adopted by different libraries to distinguish the various types of selection, locator or reference clues most convenient to their users in either scanning or filing. For these reasons, it is quite clear that, with respect to already printed cards, "the automatic conversion of files, such as the National Union Catalog, which contain considerable heterogeneous symbolism, is not likely in the near future" ${ }^{18}$ and that, with respect to future preparation, "typeface changes normally used in library output

\footnotetext{
${ }^{15} \mathrm{G}$. Chadwick, remarks, Computer Typesetting Conference Proceedings, 1965, p. 207.

${ }^{16}$ Sparks et al, 1964, p. 161-163.

17 Shaw, 1962, p. 268.

18 King, 1963, p. 20.
} 
printing of, say, the Library of Congress, would require the extensive multiplication of character sets." "19

The question remains of selecting the basic character sets for input, computer processing, and output which may differ in respect to size, nature, and coded structure, but which must be made compatible with each other or convertible from onc set to another. Indicated changes of type font, type face, point size may need to be provided for on input, innored in computer processing, and used as control eues on output. Such changes "put added burdens on the system and make it more difficult to establish a basic character set." 20

Sparks, Berul, and Waite point out further that: "Typographic composition will however present problems of encoding to handle lexical information containing character sets in multiple fonts...

"The final selection of input codes involves many complex factors including computer word length, file organization, and ease of manipulability.

"When processing text requiring character sets beyond those of the standard 47-character set of the IBM 024 or 026 keypunch, the difficulties of alphabetizing are greatly increased... It is also necessary to eliminate from the text functional codes which have no bearing on the alphabetizing sequence. ${ }^{21}$

"The method utilized for alphabetizing will have a direct effect on the interface between output printing requirements and the console display. The reason for this is that some of the possible alphabetizing subroutines are non reversible . .." 22

These difficulties will be aggravated in many documentation applications by the use of non-Roman alphabets, diacritical marks, foreign proper names, and foreign abbreviations (which may involve the use of diphthongs in authors initials), to say nothing of mathematical and other special symbols and the interpolation of other two-dimensional graphic material. For example, Barnett points out with respect to mathematical and chemical structures that "the construction of a convenient and intelligible linear representation of such formulae, that could be punched on a keyboard machine such as a Flexowriter, with a limited character set, is not trivial." 23

Regardless of the size and contents of the input and output character sets, however, in those cases involving computer processing, there are additional special problems of the internal character set which will affect sorting, ordering, filing, and interfiling. "Even though we have not yet agreed on a standard character set for our data processing computers, each computer has a single set built into it. This set is the 'natural' set of that computer, and all other sets are defined in relation to this built-in character

\footnotetext{
19 Sparks et al, 1964, p. 163.

Ibid.

Ibid.. p. 182

Jbid., p. 183

Barnell et al, 196.3, p. 270
}

set. The character set is extremely important in file definition since the character set defines the order of a file once it is sorted." 24

Sequencing records in a typographic composition system requires the application of complex alpha. betizing rules to portions of the text. These rules should be executed by the computer to provide flexibility for response to changing demands.

An examination of the textual content of records as they would appear in a typographic composition system reveals several features that would present major difficulties if the sort keys were taken unaltered from the text. Three examples are given:

1. All characters must have unique codes for proper identification during the output printing process, but some subsets of such distinct characters must be indistinguishable when used in the sort key for alphabetizing and file ordering processes. For example, in printing the following list of town and city names, the distinction between "a" and "á" must be maintained; however, in sorting these names this distinction should be ignored:
Cabot
Cáceres
Cadillac
Cádiz
Calais

If the sort processing did not treat "a" and "á" as equivalent, one must be lower valued than the other. This would give one or the other of these two sequences:

$\begin{array}{ll}\text { Cabot } & \text { Cáceres } \\ \text { Cadillac } & \text { Cádiz } \\ \text { Calais } & \text { Cabot } \\ \text { Cáceres } & \text { Cadillac } \\ \text { Cádiz } & \text { Calais }\end{array}$

Neither of these sequences is in aceord with the usual alphabetizing rules. There are, of course, many other possible examples of this.

2. Some characters in the text must be ignored when sequencing is performed. This is particularly true if precedence coding is used. A precedence code defining a change in type size must have no bearing on the alphabetizing sequence. Similarly, a discretionary hyphen should not be given a value in alphabetizing; otherwise, the results of alphabetizing would depend on characters that might never appear in the printer output.

3 . As a converse to the ignoring of various control codes, some characters must be added when part of the text is used directly as a sort key. The most obvious example of this is in the alphabetizing of proper names such as St. Andre or St. Claire. Such names are normally alphabetized as if the word Saint were fully spelled out.

Rules can of course be developed to provide the alphabetic sequences desired with at most only

\footnotetext{
Patrick and Black, I\%6t, p. 31 .
} 
minor exceptions. These rules can be written into the computer program and executed whenever the questions of proper sequencing arise. Alternatively, the rules can be executed during the processing of input data and the results appended to the record.

The program required to implement the alphabetizing rules essentially requires another code conversion. The full intermediate code would be converted to a subset of that code. It would be neither necessary nor possible to reverse the process since there would not be a one-to-one correspondence between the intermediate code and the subset code.

That part of the conversion to an alphabetizing subset code related to converting one character to another (i.e.. "A" to "a", "a" to "á" or "á" to "a") can be essentially a table look-up operation even when precedence codes have been employed. The elimination of null-valued characters, such as the discretionary hyphen, would require that one additional test be performed on each character. However, the mechanization of rules of the third type described above (i.e., equating St. to Saint) would require several extra program steps to determine when each rule applied.

As an illustration of the type of program involved in the third type of alphabetizing rule, the conversion of St. to Saint might proceed as follows:

A specification that the information field being processed contains proper names, some of which may start with such abbreviations, is included in the computer program when it is compiled. The program converts each character to its subset code in the left to right sequence. Each time an (S) is detected an abbreviation test counter is set to the value one. Each time a (t) is detected the counter is multiplied by two. Each time a period is detected the value of the counter is tested. If the counter value is two, the preceding two characters are replaced by the word Saint in its coded form, followed by a space; otherwise the counter is reset to zero. Any other character also resets the counter to zero.

This solution is illustrative only and undoubtedly not an optimum solution. It is sufficient to show, however, that converting from a full character code to an alphabetized subset code will require as complex and time consuming computer routines as will the input and output code conversion functions. This simple illustration would develop into a substantial computer routine when a full set of alphabetizing rules were included.

\subsection{Programming}

Another major problem area is that of programming. Except in a few special cases with limited requirements for versatility, programs tend to be both complex and large in the sense of demands on direct access memory. In the Government Printing Office's request for proposals for off-line mag- netic tape photocomposition, program and software stipulations illustrate some of the difficulties.

First, the equipment to be proposed should be capable of operating on the function and data codes available from a variety of computers in use and on the ASCII (American Standard Code for Information Interchange).

Next, provisions should be included for the insertion or imposition of at least 20 different graphic formats or overlays or for the generation of horizontal and vertical lines to format the output data.

Third, the proposals should cover the software support needed for system integration between the computers originating data and the high speed composition processes and equipment proposed, including the provision of manuals specifying all the detailed information needed for each specific computer to be accommodated. In particular, requirements for the preparation of magnetic tape using the format and code which will utilize all functional capabilities of the proposed composing equipment, and for core memory requirements in each computer accommodated, must be specified in detail.

The problems of compatibility in receiving tapes from a variety of computers and of telling programmers how to prepare tapes for the composition equipment were considered critical and the GPO request specified that they must be competently solved.

Still other problems of compatibility exist when the input may also include punched cards and a variety of types of punched paper tape. Barnett points out for example that: "The programs that produce... [the internal processing code] from Flexowriter, punched card and other sources have been concerned with a variety of specialized problems - special input routines for binary card images of Flexowriter and other paper tapes, accommodation of different Flexowriter codes, translation of mnemonic format requirements (macros), separation of case in Hollerith texts, and cyclic insertion of format changes in tabular and itemized material. These matters give rise to numerous scanning problems some of which are quite difficult, particularly for the last two of the topics just mentioned." 25

Programming difficulties also relate to the necessity for accommodating a wide variety of formats.

"The complexity of the control code combinations that are encountered in applications of the present program has already created a need for a higher level of input language to be converted into existing control codes by a suitable processor." 26

A programming difficulty somewhat in conflict with the typical complexity requirements is that a language is needed which will encourage good communication between programmers, originators of the data to be printed, editors, publishers, and printers. To date, this has remained a consideration with respect to further progress rather than

\footnotetext{
${ }^{25}$ Barnett et al., 1963, p. 264.
}

26 Ibid., p. 269. 
of current concern. In fact, both the Bozman and Barnett programs have made use of the FORTRAN lanquare (Formula Translation) originally developed primarily for mathematical and statistical processing applications.

Duncan at Newcastle, however, at least in terms of recent plans, intends to use ALGOL $60,{ }^{27}$ also a language originally designed for arithmetic and algebraic operations rather than for large-scale symbol manipulation such as is involved in text and bibliographic processing.

A final difficulty in the area of programming lies in the possible prematurity of programming efforts in the light of the speed with which the compositor technology has developed and is still developing. At the 1964 London Computer Typesetting Conference, for example, the question was raised from the floor: "It strikes me that a lot of people will be wondering whether it is worthwhile to enter into the computer typesetting field with existing forms of hot metal or film setting equipment when, in fact, they now know there are other forms of filmsetting coming along within the next two or three years which will tend to change the picture considerably Will ... programmes be adaptable ... or are people going to have to mark time . . . ?" 28

Several of the London participants were quite optimistic in response to this question, however, and their remarks are generally corroborated by the opinions of Barnett and Duncan, among others, in favor of proceeding promptly even in an ad hoc way. Thus, for example, ". . . I would say that 80 percent of the programming which is being done for current photocomposition will be most useful in the programming for Linotron. . ." 29

"I would agree with Mr. Corrado that a great deal of the programming which we do for computers now will be very useful, irrespective of what output unit we use." 30

"It seems that hazarding an approximation to a useful language, encoding it and trying it on real applications is the most effective way to proceed." 31

". . The best way to improve programmes for computer typesetting is to write them and use them. So far there have been so few written and used that we cannot be sure we know exactly the best way to do the job." 32

Duncan also makes the further significant point that ". . . The programmes that have been used for actual printing jobs have not been subjected to sufficient public scrutiny and measurement, nor for that matter have the styles of printing they have been designed to replace!" 33

\footnotetext{
${ }^{27}$ Duncan, 1965(b), p. 1.

${ }^{2} \mathrm{~F}$. Simmons, remarks, Computer Typesetting Conference Proceedings, 1965 p. 232 .

(1), 232

11. 232 .

V. Corrado, remarks, Computer Typesetting Conference Proceedings, 1965 p. 232 .

:3 Barnetl et al., 1963, p. 264.

atr Duncan, renarks, Computer Typesenting Conference Proceedings, 1965, p. 9.

an Duncan, Computer Typesetting Conference Proceedings, 1965, p, 9.
}

This latter point raises on the one hand questions of management implications and human factors and on the other a continuing challenge to programmers and those who design programming languages. The two foremost exponents and demonstrators of general purpose computer programs for automatic typesetting of a variety of material, Barnett and Duncan, respectively, conclude:

(1) "The definition of convenient languages for the specification of such problems to general purpose programs that could be used for mechanized documentation requires further consideration." 34

(2) "The conclusion that can be drawn now is that computer-controlled photocomposition is of practical utility, and that its further progress depends on increased communication between the writers and the potential users of the relevant programs." 35

(3) "For the future, page composition, built-up formulae, elaborate editing, and human-computer interaction to allow human judgment and aesthetics to override blind application of routine procedures will present programming challenges for many years to come." 36

"The aim must be, having once satisfied the requirements of a reasonable number of standardized formats and styles, to keep the program compilation system so simple that the instruction for a very wide variety of styles can rapidly be assembled from a library of sub-routines, which are simply called up as required from a store. Calling them up for sample pages will then be easy and economic." 37

"As the structure is being analyzed, further checking by the machine is made to determine the accuracy of the input data. The rules for rewriting structures are integrated in the program so that the computer is able to take a sophisticated look at the chemist's rewritten structure and the keypunch operator's work. It will not allow any atom to have too few or too many bonds, nor is a 7-bond permissible with atoms for which ionic bonds are not 'legal'. Improper atom and bond symbols and misplaced characters are recognized by the computer." 38

(Perhaps the programs of the future will specifically take account of variant British-American spellings, as for instance of "program" and "programme" which are obtrusive in many of the pertinent quotations cited in this report.)

"The computer can also be asked to carry out a number of original but useful checks. It can be set to recognize American and British modes of spelling and to correct one into the other. To this can be added editorial functions - such as recognition of person and tense to ensure consistency." 39

\footnotetext{
34 Barnett et al., 1963, p. 271.

as Barnett et al., 1964, p. 120

16 bid, p. 119.

: Duncan, 1964, p. 161

as Waldo, 1962, p. 2.

39 Duncan, 1964, page 160.
} 


\subsection{Implications for Management Planning, Industry Adjustment, and Systems Design}

As in many other areas of application of automatic data processing techniques and related new technological developments, a primary managerial responsibility is recognition of the need to make an overall systems planning approach, looking toward the integration of many now distinct processes, taking maximum advantage of any multipurpose processing possibilities offered and, in general, undertaking a drastic re-thinking of the possible balancings of objectives with resources now available.

The state of the art of automatic typographicquality composition is well enough advanced to underline the importance of sound system design throughout the cycle from initial recording of information to its reproduction and distribution. Because of the prospects for new system throughput capabilities, attention should be paid to the least efficient processes: multiple re-typings of manuscript copy, keyboarding operations in general, which are widely acknowledged to be the limiting factor in the economics of typesetting, ${ }^{40}$ proofreading and corrections to copy after proofing.

\subsubsection{Problems of Author-Editor-Publisher Relationships}

Just as the introduction of truly high speed reproduction processes coupled with various degrees of automation of the compositor functions has an ob. vious present or potential impact upon managerial planning and traditional practice in the publication-printing industries, so also does it have important implications for the traditional authoreditor-publisher relationships.

For example, an important aspect of the NSF grant to the American Chemical Society for the investigation of automatic typesetting advantage was: "A study of changes in the compositor-editorauthor relations that will be required as increased use is made of machines." 41

A first and obvious question is that of authoreditor-compositor responsibilities in the area of editorial changes and corrections after submission of an original manuscript, author revisions to proofcopy, the problem of proofreading generally, the requirements for proof copy, and the relative ease with which suggested editorial corrections may be accommodated easily and economically into the final printed text.

Obviation or minimization of the need for proofreading is one of the major gains expected from increased use of computer-driven typesetting systems, especially for computer-produced or compiled numeric and statistical data.

\footnotetext{
40 Duncan, Computer Typesetting Conference Proceedings, 1965, p. 8: "The (R)evoIution in Book Composition... IV," 1964, p. 67.

ution in Book Composition
Kuney, 1963, p. 249.

42 Duncan, 1964, p. 128.

43 Ibid., p. 128.

HDuncan. 1964. p. 124. also p. 50 of this report.
}

It is of interest to note that the first system concept involving programmable computing devices may have been directed at least in part to this problem:

"Some works cannot tolerate errors... and Babbage is stated to have invented the first computer, his 'differential engine,' to print navigational tables free of them. ..." 42

As first demonstrated by Bozman, the advantages are multiple. The Corliss-Bozman Monograph consisted of 559 pages of numeric data: The first few pages were carefully proofread, but when not a single error was found, it was decided to check only the first and last lines of each page, with significant gains in time and cost of such checking. Moreover, since ". . . the ordinary individual is incapable of detecting errors in large tables of figures, or in nongrammatical symbols, the advantages of automation for such work is obvious." 43

In the case of text, however, problems of user acceptance and human factors go hand in hand with prospects for new methods and media of communication-publication, new management and editorial policies and new user attitudes.

If, as has been claimed, changes suggested by an author after submission of his original scriptcopy are to be heavily penalized or refused ${ }^{44}$ (e.g., "we make the charge for corrections in the typewritten text very low but we make it very high for the final page make-up proofs ..."45), then economic factors alone may reduce requirements for proof copy in the future. On the other hand, as was remarked at the London Typesetting Conference, ". . . The printed message, often in a different format, reads differently to authors. I have found that an author is quite happy with a typescript, but seeing this in type he will want to alter it . . ."46

". . . to try and train every author in the country to accept something which probably looks to their uneducated eyes a great deal worse than the clean typescript they sent to the publishers in the first place is asking too much. . .." 47

Kuney adds: "If the traditional galley proof is to be replaced, editors and authors will need a long process of education and testing to evolve a workable basis for handling any new form of galley proof." 48

There is also a special case involving continuing need for proof copy, at least at the near to last stages, namely, where automatic typesetting methods are used to drive hot metal casting equipment. As noted by Markus: "If computer output tapes could be converted automatically to reprocopy by an errorless composing machine, there would be no need for further proofreading. A Linotype or other hot-metal typesetting machine does

\footnotetext{
45 J. J. Dikkers, remarks, Computer Typesetting Conference Proceedings, 1965 p. 41 .

${ }^{46}$ E. Houghton, remarks, Computer Typesetting Conference Proceedings, 1965 p. 41.

${ }^{17}$ R. Bennett, remarks, Computer Typesetting Conference Proceedings, 1965, p. 40 ${ }^{48}$ Kuney, 1963, p. 249.
} 
not meet these accuracy requirements, even though it can be controlled by punched paper Teletypesetting tape. The reason for this is an inherent residual mechanical error of about 2 percent even in the best hot-metal slug-casting machines. These machine malfunctions mean that copy must be proofread, because an average of one slug in fifty will have errors that require resetting." 49

Assuming that proof copy will continue to be required in at least some stages of the text preparation cycle, some of the policy questions yet to be decided include the question of when and how much proof copy will be required and whether it can be in a form other than that of the ultimate finished product.

"Will computer read-out matter in typed form be satisfactory as intermediate proofs, or will composing rooms be required to submit proofs in the type faces to be used in the book? This will bear heavily on the type of automatic equipment to be used and the new order in which operations are performed." 50

Among the solutions that have been suggested or actively tried for provision of intermediate copy in less than final typographic quality are the use of a 120 or 240 character chain on the IBM 1403 printer ${ }^{51}$ and the uncoupling of the Linotron servo to achieve an output rate of 5,000 to 10,000 characters per second, where the character quality will be the same as the final product but spacing and alignment will be irregular. ${ }^{52}$

Dikkers reports practical experience at the Royal Printing House in Holland, as follows:

"We produce a magazine which we compose on the ATF photocomposer. We use the hard copy of the Flexowriter tape punch which gives Roman and Italics in red as the proof. . . . We are trans. mitting these proofs in a facsimile fashion so that the red does not appear red. We do not have any difficulty at all with that, as they . . just want to see that all the characters which should be there are there." 53

One of the reasons for selecting a general purpose computer at Westerham Press, Ltd., was precisely for the purpose of providing such intermediate stages as a simulated galley-proof in the form of a computer print-out to which necessary corrections would be make, and, at a later stage, page proofs also in the form of computer print-out. ${ }^{54}$

Boyd suggests further that:

As far as the future is concerned, the next objective in a computerized typesetting system must be the application of a satisfactory and economic print-out device which will give a proof for correction and re-perforation of a final corrected

\footnotetext{
40 Markus, 1962, p. 28.

so "The (R)evolution in Book Composition," 1964. p. 56

st C. J. Duncan, remarks. Computer Typesetting Conference Proceedings, 1965 p. 149 .

$32 \mathrm{Yi}$

1. M. Corrado, remarks, Computer Typesetting Conference Proceedings, 1965 219

53 J. J. Dikkers. remarks, Computer Typesetting Conference Proceedings, 1965 40 .

Maclntyre, remarks, Compuler Typesetting Conference Proceedings, 1965, p. 34.
}

tape before starting the high-speed casting or filmsetting operation.", 55

Another consideration, at least in terms of possible future progress, is the question of the extent to which proofreading operations can be mechanized, perhaps through the further development of automatic character recognition techniques.

"Another possible application .. . of a page reader would be the ability to reduce proofreading. To take the original manuscript, read it into a typecomposition system, through a page reader, set the type, make galley proofs, and compare these through a page reader with the original manuscript which was stored on discs or tape, eliminates a percentage of the proofreading time." 56

\subsubsection{The Possible Role of Automatic Character Recognition}

Still another systems planning and management implication is therefore the question of the possible role that automatic character recognition equipment may play in the ongoing development of publication and re-publication processes.

It is quite probable that the reported gains in keyboarding speed, when the operator produces unjustified tape versus justified and word hyphenated tape may be substantially incremented if the operator merely types hard copy, in a pre-selected, character-reader-designed font, which would then be read automatically at rates of 300-500 characters per second, or rather faster than most available perforated tape readers today. Exceptions exist (among them, Digitronics, Omnitronics, and Rheem Electronics) but even in photoelectric punched hole readers operable at up to $1,000-2,000$ characters per second, problems of handling the paper tape and preventing its kinking or tearing point to the desirability of page readers capable of automatic recognition of characters typed on hard copy.

A unique feature of the proposed ABLE system as recently recommended to the National Agricultural Library is the plan to incorporate automatic character recognition techniques in the preparation of the Bibliography of Agriculture.

Thus, "the bibliographic citation would be com. posed as at present except that it would be typed on special paper, formatted for input to an optical scanner." 57

For full text, however, both the need for insertions of editorial changes and corrections and the inherent complexities of all the information contained in a page defy the present capabilities of automatic character reading equipment. With regard to the first point, it is noted that: ". . . In most book composition ... the machine would have to handle editing changes as well as typed matter to make the system economical. If the original manuscript must be retyped to produce a perfect copy

\footnotetext{
ss Boyd. 1965, p. 32

sh Merz, 1964, p. 85

s7 "ABLE", 1965, p. 4.
} 
for the scanner, the typist might as well create a perfect tape and avoid the scanner completely". 58 McIntosh comments even more forcefully:

"By the time you have produced a typescript sufficiently clear to be read by one of these deviceslet alone Mr. Fullick's point about the codes you have to put in - you have already reached the stage when either you can photograph that straight away or you can use the tape which you could have produced in the meantime. I can see no use whatever for reading devices in the field of printing for a long time." 59

The second point relates to even so obvious a question as the rules which would enable the machine reader to distinguish between the variant usages of punctuation symbols - a period used to end a sentence, as a decimal point or as an indication of an abbreviation. More complex is the problem that the present typographical forms of "a typeset publication are inadequate to represent content which might be required by a secondary system. For example, if one wishes to identify, in the manuscript, a referenced publication by the use of italics, the identification will be ambiguous because many other types of text items will also be represented by italics." 60

"Programming such a device will require the resolution of fantastic syntactic problems even if the machine has a universal multifont reading capability. For example, in the citation, J. Chem. Soc. 1964, 1963, which number is the year and which the page number? These are not trivial problems." 61

A third difficulty is, as in the case of output printing, the very large character repertoire required for adequate representation of scientific and technical text. Ohringer comments: "There will be many formulas and special mathematical symbols interspersed with the text. The type fonts used tend to vary greatly, including many Greek letters in addition to many styles and sizes of Roman alphabet. The page arrangement also contains too much variation for present-day optical character readers." 62

"To the extent tape cannot be produced as a by. product of copy preparation, optical character recognition techniques will probably find typesetting application as their versatility improves and their cost reduces." 63

\subsubsection{Keyboard Design and Related Problems}

Solutions to problems of character set that involve the use of a larger number of both character and shift keys for the human operator to manipulate aggravate another problem: that of the design of the keyboard itself. "The central problem of keyboard design is to get a large selection of codes from a limited, properly spaced, simple-to-operate arrangement of keys." 64

Despite the familiar arrangement of the most frequently used letters in English, the "ETAOIN" and "SHRDLU" of the linecasting keyboard, surprisingly little attention has been paid to this problem and techniques of motion study and human engineering have been little used to take into account not only the number of keys but also travel distances, size and shape of keystroking surfaces, finger pressure required, and the like.

It has been suggested that "one possible result of this type of analysis could be a bowl-shaped keyboard, with keys on the sides and rear banked up from the horizontal. . . This would enable the fingers to reach outlying keys by moving in a straight line, rather than in an arc or in two moves, over and down. Another feature would be larger keys on the outskirts, to reduce the need for accuracy in moving fingers long distance." 65

With the time required for human fingers to select and strike keys a limiting economic factor in the preparation of material for publication, various proposed techniques involve either simplifying the time-pressure requirements or multiplying the number of output operations accomplished by a single keystroke. With respect to the first of these proposed improvements, Boyd reports: "A new type of keyboard is one in which the operator starts the depression of the key manually and it is then pulled the rest of the way by a solenoid. . . This has the effect of pulling the key away from the operator's finger and it spurs him on to the next one!" 66

It is also reported that: "An experimental prototype keyboard has already been developed by Mergenthaler Linotype Company, in cooperation with the United States Army. The typewriter-style unit, known as the Army Chemical Typewriter, a triple-shift Flexowriter, produces a punched tape containing three codes for each character struck on the keyboard. One code represents the character selected and the other two record the location of the character on the page . . punched into the tape automatically. . . The codes vary with changes in position of the carriage and roller platen in the typewriter.

"The resulting tape is then fed into a computer, which stores the character codes according to location on the page. When type is set photographically, the characters are read out of the computer in line sequence. . . ." 67

As noted in an earlier section of this report, the use of either built-in spacing, justification, and automatic hyphenation processes or computer programs operating on unjustified tape not only increases keyboarding speed and accuracy but

64 "The (R)evolution in Book Composition . . IV," 1964, p. 68

65 lbid., p. 69.

${ }^{*}$ R. Boyd, Computer Typesetting Conference Proceedings, 1965, p. 157

87 "The (R)evolution in Book Composition . . . IV" 1964, p. 69. 
reduces the skill level required to that equivalent to those of a competent typist. Nevertheless, for many purposes involving large character sets, intermixed text and graphics, man-machine interaction in page display or composition, and the like, more training and new methods of operator training may be indicated. Duncan, for example, recommends: "Wc need to improve keyboarding techniques, by a reconsideration of the problems and the application of new methods of training, such as adaptive teaching machines." 68 Boyd further suggests: "Cybernetic or teaching machines exist for Army Telex operators, and a keyboard training machine would be a natural development from them." 69 Duncan concludes: "Certain it is that we need to be able to measure, assess and improve our keyboarding skills and to develop error reduction and correction routines which will improve the economics of subsequent processing." 70

The greatest promise for future development seems to be in increasing input flexibility of user terminals... We are beginning to see the use of parallel-key devices, based on the stenotype principle ..." 71

In the case of the Barnett computer-photocomposer programs, the use of program "macros" (i.e., the use of a few special codes which automatically call up control subroutines) has been noted as a means to minimize the required number of control code keystrokes. However, if in addition the keyboard itself could be designed so that a pre-determined set of codes would be punched from a single master key, it is estimated that the mix of instruction code strokes in the M.I.T. input could be reduced from 75 percent to 36 percent, a significant gain. ${ }^{72}$

Another example of multiple-output-from singlekeystroke is provided by experimental Chinese ideograph composition techniques.

"There are a number of groups of strokes which do not necessarily have meanings in themselves but which occur often in the stroking of ideographic characters. The keyboard provides for 20 of the most frequently used of these stroke groups, which have been designated entities. If such an entity is present during the stroking of a character, the operator can introduce it by depressing the proper entity key. This action introduces the complete stroking sequence contained in the entity just as though individual keys had been struck to build it up." 73

Other instances of desired changes in keyboard design that have been discussed in the literature include the provision for simultaneously printing characters along the edge of paper tape as their bit patterns are being punched ${ }^{74}$ and a capability to print in many different languages or alphabets

\footnotetext{
Buncan, 1964, p. 166.

B. Boyd, Computer Typesetting Conference Proceedings, 1965, p. 162.

70 Ibid, p. 9.

1 Ohlman, 1963, p. I94

${ }_{22}$ The (R)evolution in Book Composition ... N, 1964, p. 68.

${ }^{72}$ Pitt, 1964, p. I1-I2.

is R. Boyd, remarks, Computer Typesetting Conference Proceedings, 1965, p. 161.
}

with operator control to change rapidly from one to another. ${ }^{75}$

One of the important aspects of the overall system design program being explored at the University of Newcastle, for instance, is the analysis of keyboarding requirements, where it is hoped that psychologists will contribute to the elucidation and investigation of the "fundamental measurements ... needed before there is any real understanding of these activities." 76

It has been suggested, for example, that "another valuable keyboard capability would be the ability to set multi-column work using a single set of instructions for each column, yet producing a completely aligned and made up product from the typesetting machine." 77

\subsubsection{Implications for Systems Design}

The system throughput difficulty has been evidenced to date in various currently expedient approaches to the problems of speed versus quality, in pressures to achieve both higher speed and higher quality, and in increased recognition of implicitly required changes in management philosophy, sys. tems design, and customer attitude. Present and prospective progress, however, is indicated by the portion of multiple-purpose, integrated data processing concepts; by system designs involving multiple off-line production, and by at least some evidences of willingness to re-examine input, service and output requirements of many documentation activities.

One important management and systems planning implication is that in spite of rapid technological innovation and application, today's possibilities encompass a wide range of balanced systems. In particular, ". . . The equation becomes one of large in-plant computers for composition alone, versus large in-plant computers for combined operations, versus large computer centers, versus smaller specialized computers combined with manual operations." 78

Nevertheless, as Duncan points out: "The significant fact at this stage is that the newer systems are more flexible and so will allow a wider variety of machines to be brought into operation. The industry will then be able to buy and use the right machine for the right job, and to seek the right types of work for that particular installation, instead of equipping itself throughout with relatively inefficient general purpose machinery." 79

This means, on the one hand, that sound principles of integrated systems engineering must be employed: "To begin to understand the impact of computers on composition, it must be realized that computerized composition represents far more

\footnotetext{
${ }^{75} \mathrm{~F}$. C. Holland, remarks, Cumputer Typesetting Conference Proceedings, 1965, p. 167.

${ }^{76}$ Duncan, 1965 (b), p. 2

${ }_{77}$ "The (R)evolution in Book Composition ... IV." 1964, p. 69

${ }_{78}$ "The (R)evolution in Book Composition," 1964, p. 57.

${ }^{79}$ Duncan, 1964, p. 123.
} 
than an improvement in the setting of type. Com-

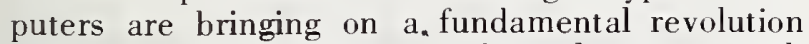
in composition. Studies and applications work already have shown that the real economies lie in reorganizing the composition process-not in merely trying to superimpose computers on present systems." 80

On the other hand, it also needs to be recognized that: ". . . whatever the technological possibilities may be their exploitation involves re-orientation of management policy to a drastic degree." 81

The problem of re-thinking the processing-printing complex arises in terms both of balancing input with high speed in intermediate or final operations and of balancing output with rates of high computer processing.

With respect to the balancing of final output capabilities with high speed computer output rates, an obvious solution is the adoption of multiple offline production systems. Thus, "the terrific speed at which computers produce tape raises some problems. The RCA 301, for example, turns out enough TTS tape to fill a whole newspaper column in 17 seconds, so fast that conventional typesetting equipment can't begin to keep up and batteries of machines must be used." 82

Such batteries may be operated either on-line or off-line through the use of buffers and allocation devices. At The South Bend Tribune plant, for example, "Under guidance of the IBM 1620 computer the buffer selects from the six Harris Intertype Monarch linecasting machines one which is set up for the proper type font and size and column width for the story. These specifications are marked by the editor on the original copy and punched into the first tape along with the copy." 83 Similarly, at The Kansas City Star, an IBM 1620 installation is linked to an allocating capability which feeds twelve linecasting machines.

With respect to multiple on-line production capabilities Duncan reports, "In 'on-line" operation the processing computer is connected directly to a battery of tape-operated line-casters which are setting copy as it comes through. By the use of allocators each machine can be kept loaded with work appropriate to it, and in case of difficulties automatic re-routing to another machine or to a high density magnetic tape storage is possible." 84

This advantage is claimed to be especially valuable when typesetting equipment can be set up so that different machines need handle only a few fonts at a time. A further advantage is the capability of switching from one type of equipment to another to meet production schedule demands. Thus, at Westerham Press it is claimed that: "Within our system we can output to a standard line-caster, or to a photosetter. If the job being computed has

\footnotetext{
80 "The (R)evolution in Book Composition," 1964. p. 56.

${ }^{81}$ Duncan, 1964, p. 166

B2 Blundel, 1964, p. 28.

${ }^{3}$ Goodwin, 1964, p. 34.

Duncan, 1964, p. 146.
}

to change from Linotype Baskerville to Monotype Baskerville, it is possible to recalculate and come out with a tape which after subsequent conversion goes on to a Monotype unit. This may be of only academic interest but we are particularly interested in this for controlling production in a large printing group, where we may have an urgent job which, originally scheduled for one piece of equipment, could be switched to another unit." 85

Other claimed advantages of allotment to a number of different machines are that it enables an operator to control machine loadings independently of the rate of tape output ${ }^{86}$ and that down-time and changeover-time on the typesetting equipment itself can be reduced. ${ }^{87}$

Another important aspect of planning and systems design for multiple off-line production is that of providing for such production at remote locations by tie-in to communication networks. This is already evident in a number of news service applications, where, for example, the Associated Press is using the Data-Phone to transmit justified stock market copy to its customers throughout the country. As Blondeau notes, "this is on the wire minnutes after the markets close in New York." 88

With respect to prospects for future progress for multiple printing at remote locations, Duncan suggests: "As the onset of film composition, of punched tape, or of thin presensitized litho plates reduces the bulk and weight of the work content of printing it becomes a simple matter to convey it rapidly all over the world by jet aircraft, or to transmit it practically instantaneously by communications satellite, or coaxial telegraph cable." 89

Still another advantage of the communication network tie-in is likely to be a quick and reliable depository system for scientific and technical material (both bibliographic data and full text) in reproducible form at many locations. For safety and security reasons also, "simultaneous replication of the printiny surfaces at several locations will be an attractive variation on present-day practice." 90

Multiple inputs to a single high speed processor are also a part of the system design equation. The French CAE system provides for both a double input and a double output which not only increases the throughput speed but also allows the computer, when required, "to accomplish simultaneously two different tasks on independent texts." 91

Another example is the plan for the Thomson Computer Setting System in Great Britain. "Unlike existing computer type setting methods which are being developed in the United States, the new British system allows for direct communication between the operator and the computer.

"The twelve keyboard operators will share the one computer and use it as their slave to justify

\footnotetext{
ss MacIntyre, remarks, Computer Typesetting Conference Proceedings, 1965, p. 35. "s6 "The (R)evolution in Book Composition . . . IV", 1964, p. 70.

${ }^{87}$ Duncan, 1964, p. 162.

Blondeau, 1963 , p. 89

a9 Duncan, 1964, p. 121.

${ }_{90}^{89}$ Duncan, 1964, p. 163.

${ }^{91}$ G. Bafour, remarks, Computer Typesetting Conference Proceedings, 1965, p. 195.
} 
lines, 10 remember type faces and sizes, to store copy for corrections, to construct tables, to identify paper tape and to hyphenate words. Each operator will give his own instructions independently to the computer. The computer will immediately tell the operator if he makes a mistake or gives an impossible instruction. If a mistake has been made the operator can remove it with a touch on the keys." 92

Some of the practical problems in sound systems planning for the introduction of semi-automatic or automatic techniques in the composing room or printing plant include such minor but significant details as the following: "One of the unexpected problems which has occurred is that of winding and unwinding tapes. The photo-electric readers are very sensitive to tight or kinked tape, and if either fault develops the output tape can soon become nonsense. This is the sort of thing that one tends to ignore when one thinks about computers but it can very quickly make a loss out of a profit." 93

Even in relatively slow, relatively unsophisticated equipment systems, such as Linasec I, ". . . The standard clockwork tape winder available in this country is about $5^{\prime \prime}$ or $6^{\prime \prime}$ in diameter, and you have to change these in a remarkably short space on Linasec [I] when you are processing at 100 a second." 94

Another managerial question in terms of an integrated systems approach is that of multiple use, in the same organization, of the capabilities of automatic data processing. First, it is to be noted that "most organizations that are of a size and structure that warrant a large computer are also of a size and structure that could benefit from an 'in-house' facility for the rapid production of varied typography." 95

Secondly, despite the early failure at the Arizona Journal, there are definite prospects that computers available for typesetting purposes may also be used for other productive operations in the same organization. Thus, in one of the on-going newspaper applications, "in addition to using this equipment for composition, Perry also uses it for accounting operations of some 26 or 28 different units of the organization." 96

An important change that is promised is in the area of storage requirements for material that is to be revised, corrected and/or cumulated, and reissued.

"One trip through the mountains of metal stored in the plants of most large commerical and book printers drives home the extremely uncommon utility of compact data storage on tape." 97

In such applications as revised and updated directories, gazeteers, cumulative indexes, recurrent bibliographies and the like, the storage prob-

\footnotetext{
92 The Computer Bulletin 9, 19 (1965)

${ }^{9}$ Boyd, 1965, p. 28.

93 Boyd, 1965, p. 28.

${ }^{2+4}$ R. Boyd, remarks, Computer Typesetting Conference Proceedings, 1965, p. 38.

I.uce and Barnett, 1963, p. 293.

4os Touman, 1963, p. 105.

6. "Computerized Typesetting," 1964, p. 5 .
}

lem for hot metal is notoriously severe and the re-arrangement procedures are slow, costly, and cumbersome. With the use of photocomposition techniques, storage is much more compact and manageable, but the page revision problems are even more critical. As Duncan remarks:". . . Until recently there was no alternative to storing metal and updating it by substitutions, or, if alterations above say 10 percent were involved, by resetting. The use of film or flat photo copy instead of metal did not in general make things easier, as it was less durable and just as difficult to match in as a mixture of new and used hot metal type." 98

The updating and reissue problems constitute one of the principal reasons for high speed requirements in semiautomatic and automatic type composition systems. For example, in typical directory composition applications,". . . you have several hundred listings on a page. If you want to re-set that page and add three names and take one out, the keyboarding which has to be done involves only three lines, but the new machine has to be fast enough to set the other 300 or 400 lines which are on the page. . . "99

At least two special factors may also require recognition in managerial re-thinking of the preparation-processing-publication cycle as the result of the introduction of new techniques. One of these is the question of special copyright considerations, and the other is the related one of ownership of products eventually to be sold, especially where processing and packaging has been shared among several different organizations. Duncan asks: "One complex matter will arise when tape is prepared in one place for processing to saleable copies at another. To whom will it by law belong, and by whom by trade custom can it be used?" 1

Copyright considerations may relate first to the substance of material reproduced, specifically including the use by abstracting services, whose output is copyrighted, of an author abstract which may also be replicated by another organization either as part of its own accession announcements, as part of a storage, search and retrieval system or as part of a demand bibliography.

Next, there is the question of copyrights on material essentially venerated, not merely compiled, by machine systems. This is complicated by the general question of copyrights in translations, especially if the translation is the output of a machine translation program. Further complications arise from the fact that computer programs as such may now be copyrightable under certain conditions.

"There is one final problem that may retard and damage this whole new expansion-that is the problem of copyright and of regulations governing the use of text in computer form." 2

\footnotetext{
94 Duncan, 1964, p. 147.

49 V. M. Corrado, Computer Typesetting Conference Proceedings, 1965. pp. 235-236.

Duncan, 1964, p. 161

Kehl, 1965, p. 26.
} 
Another highly specialized, but potentially troublesome question, is that of copyrights in the typography itself (covered, for example, in the British Copyright Act of $1956^{3}$ ). If the original typography of all or part of copyrighted material is accidentally or otherwise exactly reproduced in subsequent reprocessing and resetting is there a potential infringement? If the typography of a first printing, whether of primary or secondary publication, is computer-produced, what copyrights if any, can be lodged with author, publisher, computer programmer, composition service bureau or printer?

It has also been demonstrated that facsimile transmission may be used for sending copy and proofs to and from various locations. For example, the Portland Printing Company has used Western Union's Broadband Exchange Service for transmission between New York and Boston at rates of one page in four minutes, thereby becoming able to "move copy immediately to our plant, set in type and return out of town proofs within a single working day." 4

\subsubsection{Implications for Industry Adjustment}

In the preceding section, we considered some of the implications to be found in the literature of automatic type composition with respect, primarily, to the design of a single system or to the adoption of the new techniques by a single organization. There are, in addition, some implications likely to affecting the printing and publishing industries as such and thus to point toward requirements for industry-wide adjustments.

First is the question of new publication, reproduction and distribution possibilities. These imply the desirability of looking toward radical re-orientation of publication and dissemination policies and toward the introduction of new types or means of reproduction and distribution.

First, "As printing becomes increasingly involved in data processing techniques, so is publishing involved in the tremendous developments taking place in information retrieval and dissemination... The operation of powerful information retrieval systems may (as in the case of MEDLARS) produce more or different printed matter replacing some of the books and pamphlets already in production." 5

In a study of desired improvements in the communication network for biomedical information, pilot trials have been recommended for: ". . . using advanced techniques in the publication of established biomedical journals, e.g., computer composition, 'phototypesetting', microform editions . . ."6

As the Weinberg Panel report suggests, "the invention of the new retrieval methods is beginning

${ }^{3}$ Reed, 1960, p. 162.

+ CIS Newsletter, Aug 1, 1965, p. 4.

s Duncan, Computer Typesetting Conference Proceedings, 1965, p. 11

"Communication Problems in Biomedical Research", 1963, p. 21. to affect our traditional modes of communication. The traditional forms of the book, journal, and reprint may eventually give way to the machine storage of graphical and digital information and machine-generated copy." 7

Material not now usually typeset - the technical report and the wide variety of computer-compiled or computer-generated products - is likely to be increasingly fed to typesetting equipment in the near future. As Kraft remarks: "Today, the newer developments in typographic composition and reproduction-particularly the development of highperformance phototypesetters and tape-controlled linotypes-have increased the speed and reduced the cost of typesetting to such an extent that typeset copy can compete with other methods of document reproduction in speed and cost." 8

High typographic quality of the bibliographic information may make more feasible the suggestion of Markus that there should be explored "the economic aspects of printing indexes in much smaller type and furnishing a magnifying glass with each volume." 9

Demand publication (whether of original text, supplemental data and appendixes, or specialpurpose bibliographies and index-document listings) imposes additional requirements-often of small production or replication runs and often requiring highly specialized selection-extraction and re-assembly operations. In this area, a suggestion of Duncan's indicates some of the ner technological possibilities: "When the number of offprints required is small then some cheap system of shortrun production is more appropriate than full-scale printing. In these circumstances the photocomposer, or an attachment to it, can compose reprints directly on to a photosensitive short-run plate, or some intermediate suitable for inexpensive conversion." 10

With respect to the printing industry, the clearly implied requirements for industry-wide readjustments range from re-thinking of criteria for accepting jobs and for determining an economic size for a printing run, through the problems of labor relations to the question of education and training of those now engaged in printing operations and of those who will enter the industry in the future. Underlying all these considerations is the recognition that: "There is ever increasing demand for cheap printed matter of good quality. These demands cannot be met by old craft methods." 11

Turning to the mechanics of production of both the traditional and newer forms of publication; Duncan as one of the principal exponents and students of the impact of the new techniques points out that: "The concentration of enormous productive power in a small unit, such as a digital computer or an ultra fast photocomposing machine,

\footnotetext{
7 President's Scientific Advisory Committee, 1963, p. 32

${ }^{8}$ Kraft, 1963, p. 275.

${ }^{9}$ Markus, 1962, p. 21.

${ }^{10}$ Duncan, 1964, p. 162.

${ }^{11}$ Harrison, 1964, p. 1.
} 
inevitably changes the balance of the machines that feed it and those that it feeds. This poses wreat problems of production while completely changing the method of operation and the balance of the plant in use. I believe it also implies, at least to start with, a change in the variety and style of the operations to be performed." 12

Elsewhere, Duncan provides a comprehensive coverage of the problems of printing industry adjustments as follows: "The pressures operating in the printing industry and the deficiencies in means to combat them ... may be summarized as follows:

1. Increase in the total quantity of printed matter required.

2. Increase in the speed at which material is required and in its complexity both of content and design.

3. Increase in the speed with which copy is being fed to composing machines (from wire and business tape and from computers).

4. Increase in production associated with modern high speed rotary presses.

5. Drive for greater productivity ...

6. Competition from newer media, and non-traditional methods.

7. Introduction into the industry of work study ... .

8. Low per capita expenditure on research in the industry as compared with newer growth industries.

9. Low percentage of technologists (as distinct from arts graduates) employed in the industry.

10. Meagre facilities available for advanced instruction and research. ..."13

A more specific, but exemplary, problem of industry-wide concern is that of lack of compatibility between systems and hence the need for conversion capabilities for some years to come.

"A considerable number of code translating machines will be necessary to handle the multifariously coded systems at present in use, and TTS tape, with its unfortunate non-standard sprocket hole position, will have to be converted for processing on general purpose systems." 14

One obvious question is that of possible standardization of perforated tape codes, formats, and processing characteristics.

Duncan suggests that: "We need a moderately increasing degree of standardization, first over the machine processing methods and codes, then over the variety of printing formats and styles." 15

It is also to be noted that ". . . it is likely that we will have to cope with a wide variety of inputs and formats for some time to come... The use of code converters, however, requires very careful attention to code combinations and to the

\footnotetext{
${ }^{12}$ C. J. Duncan, Computer Typesetting Conference Proceedings, 1965, p. 3.

${ }_{13}$ Duncan, 1964, p. 126

14 Ibid., p. 130.

is Ibid, p. 166-167.
}

reservation of codes for control functions and format and geometry of the paper or magnetic tapes." 16

Further, ". . . large amounts of machinery in use today will either have to be altered or replaced, or we shall have to go on using converters and translators for a considerable time, and at considerable expense. The printing industry, particularly the newspaper industry, is the worst off of all from this point of view because practically all the tape operated plant it uses has its own private code, and its own 'tape' format." 17

The fact that presently proposed standardized codes such as ASCII or the even more restricted British-International 6- and 7-bit codes cannot adequately cover the character repertoire required for scientific and technical material, except with extensive use of "escape" characters or precedence coding has been mentioned previously. Finally, it is to be noted that ". . . even if a common language tape could be agreed upon in printing, converters would still be needed to connect computerized composition systems with outside sources of material." 18

Recognizing the need for code standardization for newspapers, the American Newspaper Publishers Association in the summer of 1965 nominated Mr. Harold R. Hoots of the Lindsay-Schaub Newspapers, Decatur, Illinois, to serve as a member of the American Standards Association's Subcommittee X3.2, Codes and Input-Output, and Task Group X3.2.4, Code Development. The Mergenthaler Linotype Co., has had representation on both of those groups for two years, in the persons of $\mathrm{O}$. R. Arne, now with the New York Daily News, and R. J. Donahue.

The need for standardization in this field is evident, and a substantial development effort is indicated, in order that the 96 graphic symbols and half dozen format effectors of ASCII can be extended, in a standard manner, to encompass all of the typographic symbols and controls required in the composition of newspapers.

\section{Summary: The Present State-of- the-Art and Future Prospects}

The state-of-the-art is well indicated in summary by the following. In June 1963 the U.S. Government Printing Office issued a "Request for Proposal for Off-line Magnetic Tape High Speed Photocomposer," which required:

a. typography and formatting by computer processing.

b. computer programs to accomplish this.

c. the use of computer-generated magnetic tape, in several existing tape formats, as off-line control and information input to the photocom. poser.
16 Sparks et al, 1964, p. 182.
17 Duncan, Computer Typesetting Conference Proceedings, 1965, p. 10.
ix "The (R)evolution in Book Composition... II", 1964, p. 70. 
d. the production of photographic image pages (8 810.5 inches) of graphic arts quality.

e. composing in full image width (8 inches) or in two columns, one column at a time.

f. a minimum output speed of 600 characters per second of six point type (lower for larger type).

g. $5,6,7,8,10,12,14$, and 18 point size values.

h. proportional character spacing, computed.

i. justified lines, computed.

j. variable line spacing.

k. horizontal type width condensation under code control to $85 \%$ and $70 \%$ of true width, without reduction in line length (optional).

1. multiple type faces, mixed without restriction.

$m$. electronically controlled inclination of the vertical axis of characters as a means of simulating italics (optional).

n. electronically controlled "stroke width" of characters as a means of simulating bold face characters (optional).

o. resolution of 250 line pairs per inch at a density ratio suitable for platemaking.

p. reduction to one-fourth and one-eighteenth size image areas (optional).

q. at least 20 different graphic overlay formats to be inserted on code command in random order, or means of generating horizontal and vertical lines, to closely specified tolerances, in response to code command.

$r$. operation in various existing magnetic tape codes with transition to the American Standard Code for Information Interchange (ASCII).

Sufficient interest in the above "Request for Proposal" was evidenced, despite the severe requirement, that a Bidders Conference held at the Government Printing Office in August 1963 was attended by representatives of the potential suppliers shown in Table 3.

TABLE 3. Companies represented at a Photocomposer Bidders Conference at Government Printing Office on August 12, 1963.

*1. Alphanumeric, Inc.

2. American Type Founders Co., Inc.

3. Aries Corp.

4. Arnoux Corp.

5. Auerbach Corp.

6. Burroughs Corp.

7. Data Display, Inc.

8. Di/An Controls, Inc.

*9. A. B. Dick Co.

10. General Dynamics Electronics

11. General Electric Co.

12. General Precision, Inc.

13. Harris Intertype Corp.

14. Houston Fearless Corp.

15. Information Dynamics Corp.

16. Inforonics, Inc.

*17. International Business Machines Corp.

18. Itek Corp.

19. Lanston Monotype Co.

20. Litton Systems, Inc.

*21. Mergenthaler Linotype Co.

22. Philco Corp.

*23. Photon, Inc.

24. Radio Corp. of America

25. Shaza Industries
Several other companies are known to be engaged in research in photocomposing. The companies marked with an asterisk (*) submitted formal pro. posals in October 1963, and the final award of a contract was made a few months later to the Mergenthaler Linotype Co., for a photocomposing system based upon its "Linotron" all optical-electronic equipment developed in collaboration with CBS Laboratories.

In terms of technical developments as of August 1965, certain conclusions as to the current state of the art and the prospects for further progress can be drawn. It should be noted, however, that the available and developing technology is such that several different approaches may, as appropriate, proceed concurrently but independently.

First, it should be recognized that the computer, per se, is not necessarily linked to a single high speed composition technique.

It can be claimed that, "automated techniques are applicable to all typesetting methods, although the speed and flexibility of photographic composition provides the most fertile avenue for fully exploiting computer capabilities." 1

Nevertheless, "many people believe that computerized composition will greatly accelerate the development of photocomposition methods and the replacement of hot metal. Others, however, argue that hot metal has a great deal to offer in computerized composing rooms, and it will continue to find a major role in the future." 2

On the other hand, it has been recognized that, "although only one facet of the total picture, the computer has somehow become the symbol, if not the synonym, for automation." 3

There are several good reasons. The most spectacular of the semi-automatic and automatic aids to composition and typesetting is certainly the general purpose digital computer, which has worked its way into nearly all aspects of human activity: business, industry, commerce, science, technology, government, education and the professions.

The computer, then, in one of its many roles, has been gradually and painstakingly assuming an increasingly important position as skilled compositor, to which it is rather ideally suited, since this task involves an enormous repetition of well-defined decision processes. The computer has already demonstrated its ability to eliminate the need for the complex compositor skills from the keyboard operator, to provide an enormous increase in the speed of the compositor functions, and to keep pace with developments in electronic photocomposers which promise typesetting of graphic arts quality at speeds of thousands of characters per second, intermixed with electronically photocomposed line art, screened halftones and continuous tone illustrations.

There is, in addition to high speed typographic quality in black and white, some promise of color

\footnotetext{
1 "Computerized Typesetting", 1964, p. 4.

2 "The (R)evolution in Book Composition . . . III", 1964, p. 61.

3 "Computerized Typesetting", 1964, p. 2.
} 
as well. While, as of 1963 , a Mergenthaler team was pessimistic ("with respect to color capability, the investigations show that there are no practical means, methods or mediums available now or in the near future that will simplify or circumvent the complicated conventional techniques and mediums currently employed for high quality color imaging and reproduction." "4), a practical demonstration of at least limited application of color has already been achieved: "Art and electronics were interestingly combined to produce the cover on the July [1965] issue of Fortune magazine. The designer's specifications were converted to a computer program, punched into 8-channel paper tape and generated on an oscilloscope via a PDP-1 computer. Multiple photographic exposures through two filters added color to the electron tube images. . . .5

Thus the present prospects for automatic, high quality typographic composition at high speed show progress toward such specifications as outlined by Ohlman at the 1961 Annual Meeting of the American Documentation Institute: "What we require is a device that can create a visually attractive page for a competitive price at reasonable speed. It should provide a full variety of type fonts, the gamut of colors, and automatic control of placement and shape, as well as the ability to compose analog matter, such as graphs and pictures, from digital inputs." 6

As we have seen, there are two complementary aspects of the current state-of-the-art in automatic typographic quality composition. They are not necessarily interdependent. Computerized typesetting can be applied as well to hot metal equipment control as to photocomposers and, by suitable planning for multiple off-line production, to relatively slow speed composers and printers and still achieve significant throughput advantages. Conversely, high speed photocomposers may or may not be computer-driven and yet portend significant gains in economy of printing and in timeliness of publication.

Where computers are already involved in the preparation-to-publication cycle, it is reasonably certain that they can also be effectively used to assure high typographic quality of finished products.

However, it is with the combination of high speed photocomposition and computer typesetting control that the most significant gains are beginning to be realized. Some of these are already claimed to be worth as much as the cost of the equipment itself. These include, first, space savings resulting from fewer pages at lower cost, estimated at up to $7.5 \%$ space saved for the same text in newspaper composition (for example, "significant savings, reported to be up to $5 \%$ in the case of the Oklahoman, which will go a long way towards paying for the computer ..." ? far higher savings in the case of print- ing of material produced as a result of computer processing. Thus Forbes remarks:

"Reduction in the book bulk is achieved by getting two pages of information on to one page. Higher readability will generate sufficient savings to pay for the major portion of the initial programming."

The future potential in this area is emphasized by the recognition that in the United States alone, "500 million pages a month are produced by high speed line printers." 8

Significant gains in the bottleneck areas of key. stroking and proofreading have been discussed earlier.

Related research potentialities to be explored include the further development of automatic character reading techniques suitable for use in integrated text processing systems and in further investigations of "the possibility of eliminating writing or typing as a means of originating material is now available in crude form, in the voice-writer, the IBM 'shoebox,' and a similar machine by RCA." 9

As is to be observed with respect to labor relations problems that have already occurred, the implications of the current state of the art of automatic typographic composition are already affecting the question of training and skills required in the composing room and the printing plant. On the one hand, there is the fact that skilled typists producing unjustified tape for subsequent machine processing can replace the skilled compositor in many applications. On the other hand, there are new challenges to the industry to become familiar with and learn to take advantage of the new technological advances.

"As typesetting... moves from mechanics to electronics and a host of sophisticated, alien disciplines, the changes in the approach toward related education and training must be no less radical." 10

Current progress has been hailed as a "movement from sweat to sophistication [and] more efficient systems of printed communication are evolving from the cooperative assault on the problems of typesetting by experts in both printing and electronics." 11

Finally, as several of the pioneering users of the photocomposition-computer combined capabilities have summarized:

"Considered as a typographic quality output device, the tape driven Photon bypasses several cycles of manual keyboard action, proofreading and correction, when the results of a computation are to be published. It provides a means for increasing the readability of results when manual typesetting would not be cheap or fast enough." 12

"Probably the most important improvement, however, is in readability. The printing is not only clear and distinct, but the availability of a choice

\footnotetext{
* Mergenthaler. 1963. p. 17.

CIS Newsletter. August 1, 1965, p. 4

Herbert Ohiman, panel discussion of Markus, 1962, p. 25

'Duncan, 1964. p. 155.
}

8. Schwartz, remarks, Computer Typesetting Conference Proceedings, 1965, p. 76.
"Duncan. 1964, p. 131.
19 "Automation and Education," 1965. p. 1.
"1. Computerized Typesetting." 1964, p. 3.
12 Luce and Barnett, 1963. p. 293. 
of type styles, mathematical symbols, special symbols, and Greek and italic characters to fit each particular job contributes toward the publication of data which is easier to understand and a pleasure to read and use."'13

Duncan warns, however, that:

"The most that can be said for our efforts so far is that they have shown the great extent of the ground work that needs to be done before their successful use can be assured." 14

Many questions of operational analysis, human factors, and user acceptance have not yet been investigated to any significant extent. In the first place, many aspects of these questions involve subjective or qualitative or even aesthetic judgment, for which no quantitative measures have yet been devised. In the second place, even where quantitative measurements are feasible, they have seldom been developed and applied. Duncan (1964) reports, for example, on critical reactions to machinecontrolled interword spacing in the range of 4 to 12 "M" units. He notes that: "All typographers so far consulted consider closer spacing down to 3 M.U. minimum preferable to occasional lines with wider spacing and would prefer an upper limit of about 9 M.U. . . . We must report an actual count of the interword spacing used on 958 lines by a most respectable publishers' printer. $9.4 \%$ of the lines had spacing over 9 M.U.; $4.5 \%$ over 10 M.U., and there were two lines over 13 M.U. So much for the

\footnotetext{
${ }^{13}$ Buzman, 1963, p. 4

14 C. J. Duncan. Computer Typesetting Conference Proceedings, 1965, p. 2.
}

practical solutions adopted by working compositors, and apparently disregarded or unobserved by readers and designers!' 15

How do the problems of improper hyphenation of such and such a percentage of the number of hy. phenated lines compare as between the usual readers of the newspaper, the typewriter-copyreproduced technical report, the computer-compiled KWIC index, the periodical article, the full text of a book on scientific and technical topics?

Again, how serious is an occasional ragged-rightmargin line? Is it better or worse with respect to overall customer acceptance than an occasional (more frequent?, less frequent?) improper or ludicrous hyphenation?

This is but one example of many such questions yet to be explored, and demanding early attention. McIntosh, for example, makes the following pertinent comments:

". . I wonder whether a very small sum of money by comparison with the hundreds and thousands of pounds which are going into this technology at the moment might be devoted to some fundamental study of readability, typefaces, sizes, hy. phenation, justification and supposed standards of typography. If that study were undertaken on a national basis or an international basis - which, as far as I know, has never been attempted-it might eliminate a lot of the problems into which a tremendous amount of effort is going." 16

${ }_{16}^{15}$ Duncan, 1964, p. 155.

${ }^{16}$ A. C. McIntosh, Computer Typesetting Conference Proceedings, 1965, pp. 149 150 



\section{Bibliography}

1. Addressograph-Mulitgraph Corp. A-M Model 960-High Speed Line Printer. Manual of Operation, Cleveland. Ohio. 1961. 19 p.

2. Addressograph-Multigraph Corp. Light Harnessed to Your Paperwork Needs with Electronic Printers by Addressograph-Multigraph. Descriptive Brochure. Cleveland. Ohio.n.d., 6 p.

3. Alden. W. L. Cutting Communication Costs with Facsimile. Data Processing Magazine 6, 11-14 (Sep 1964).

4. Allen. G. S. A Study of Electronics for Printers, Graphic Arts Progress 9, 1-5 (July-Aug 1962).

5. Allen, G. S. Preface, in Institute of Printing, Computer Typesetting Conference, Report of Proceedings, 1965 , pp. vii-viii.

6. Alphatype-Filmotype Corp. Alphatype Photo-Typesetting Facts. Descriptive Brochure. Skokie, Ill., n.d., 4 p.

7. American Documentation Institute. Parameters of Information Science (Proceedings of the Annual Meeting. Philadelphia, Oct 5-8. 1964), Vol. 1, W ashington. D.C.. 1964. $521 \mathrm{p}$.

8. (American Federation of Information Processing Societies)*. Review of Input and Output Equipment Lsed in Computing Systems. Joint AIEE-IRE-ACM Computer Conference, 1952, Vol. 2. American Institute of Electrical Engineers, 1953, 142 p.

9. (American Federation of Information Processing Societies)*. Proceedings of the $W$ estern Joint Computer Conference 1955, Vol. 7. Institute of Radio Engineers. Inc., New York. 1955. 132 p.

10. (American Federation of Information Processing Societies)*. Proceedings of the Eastern Joint Computer Conference 1956, Vol. 10, American Institute of Electrical Engineers. New York. 1957, $149 \mu$.

11. (American Federation of Information Processing Societies)* Proceedings of the Eastern Joint Computer Conference 1957. Vol. 12. The Institute of Radio Engineers. New York. $1958.259 \mathrm{p}$.

12. American Federation of Information Processing Societies. Spring Joint Computer Conference, 1963, AFIPS Conference Proceedings. Vol. 23, Spartan Books, Baltimore. Md.. 1963. $413 \mathrm{p}$.

13. American Standards Association. American Standard Code for Information Interchange. ASA Standard X 3.41963. New York. 1963. 11 p. See also. Proposed Revised ASCII, Communications of the ACII 8, 207-214 (1965).

14. American U'niversity. Machine Indexing: Progress and Problems (Papers presented at the Third Institute on Information Storage and Retrieval, February 13-17. 1961). Washington. D.C., 1962, 354 p.

15. American Type Founders Co.. Inc. ATF High Speed Photographic Typesetter Model B, Descriptive Brochure, Elizabeth, N.J., n.d., 16 p.

16. An Approach to Cost-Cutting in the Printing of Supply Catalogs. Text of an audio-visual presentation prepared by the U.S. Navy, Publications and Printing Service, 1962.

17. Anderson, H. E. Automated Plotting of Flow-Charts on a Small Computer. Comm. Assoc. Computing Machinery 8 , 38-39 (1965).

18. Anger, I. J. and J. E. Reeves Fowkes. Evaluating Phototypesetting Systems: A Basic Approach. Book Production Magazine 79, 68-72 (June 1964).

19. Anthony, L. J. and J. E. Hailstone. Use of Punched Cards in Preparation of Lists of Periodicals. ASLIB Proc. 12, 348-360 (1960).

20. Arnett, S. E. Electrophotography for Graphic Reproduction, The Graphic Arts Monthly 34, 44-49 (Feb 1962).

21. Ashworth, B. P. Refresher Course: The Historical Background to Line Casting, British Printer 74, 132-136 (Sep

* Note that although Proceedings of the Joint Computer Conference were not pubNote that although Proceedings of the Joint Computer Conference were not pub-
lished by the American Federation of Information Processing Societies prior to Volume 20 , they are here grouped in accordance with the volume series numbers.
1961); Construction of Machines for Linecasting. British Printer 74, 132-136 (Oct 1961).

22. Automatic Japanese Filmsetter Sapton-N, Asian Print 4, 70-71 (1961).

23. Automatic Typesetting (First International Technical Information Congress), La Compagine Francaise d'Editions, Paris, $1965,374 \mathrm{p}$

24. Automatic Machine Composition, Litho-Print 4, 201-203 (1961).

25. Automation and Education, CIS Newsletter. 1 Aug 1965, p. 1.

26. Bafour, G. P. A New Method for Text Composition: the B.B.R. Systems, Printing Technology 5, 65-76 (Nov 1961).

27. Bafour, G. P. Vues d'Ensemble sur Les Problemes Actuels et Futurs de la Composition Automatique des Textes, in Automatic Typesetting. 1965, pp. 13-37.

28. Bafour. G. P. La Composition des Textes par les Techniques du Calcul Electronique. Syndicat B.B.R.. Paris, 1958.

29. Bafour, G. P. Le Procede B.B.R. de Composition Automatique des Textes: Principes du Procede et Experiences Preliminaires. Syndicat B.B.R.. Paris, 1958.

30. Bafour, G. P. Le Procede B.B.R. de Composition Automatique des Textes: Specimens, Epreuves et Commentaires Relatifs aux Essais Preliminaires. Syndicat B.B.R., Paris, 1958.

31. Bafour, G. P., A. R. Blanchard and F. H. Raymond. Automatic Composing Machine, U.S. Patent 2,762,485. Patented 11 Sep 1956; filed 21 Mar 1955; (Class 197-20).

32. Bagg, T. C. and M. E. Stevens. Information Selection Systems Retrieving Replica Copies: A State-of-the-Art Report, NBS-TN 157, National Bureau of Standards, Wiashington. D.C., 31 Dec 1961, 172 p.

33. Ball, H. Bureau of Ships Rapid Selector, Ships Journal 10, 6-7 (1961).

34. Barnett. M. P., et al. Composed by Computer, Ferranti Computer World, No. 16. July-Aug 1963.

35. Barnett. M. P. and K. L. Kelley. Computer Editing of Verbal Texts. Part 1, The ESI System, Amer. Documentation 14, 99-108 (1963).

36. Barnett, M. P., K. L. Kelley and M. J. Bailey. Computer Generation of Photocomposing Control Tapes. Part 1: Preparation of Flexowriter Source Material, Amer. Documentation 13, 58-65 (1961).

37. Barnett, M. P., D. J. Moss and D. A. Luce. Computer Generation of Photocomposing Control Tapes. Part II. The PCG System, Amer. Documentation 15, 115-120 (1964).

38. Barnett, M. P.. D. J. Moss, D. A. Luce and K. L. Kelley. Computer Controlled Printing, in American Federation of Information Processing Societies, Spring Joint Computer Conference, 1963, pp. 263-288.

39. BaxendaIe, P. B. Machine Made Index for Technical Literature: An Experiment, IBM J. Research and Development 2, 354-361 (1958).

40. Benson-Lehner Corp. Descriptive Summary BensonLehner/Transdata 944 Ultra-High-Speed Microfilm Printer-Plotter, Publication No. 492 A. Van Nuys, Calif., Feb 1963, 9 p.

41. Bessinger, J. B.. Jr., S. M. Parrish and H. F. Arader [eds]. Literary Data Processing Conference Proceedings September 9, 10, 11, 1964, International Business Machines Corp., White Plains, N.Y.. 1964, 329 p.

42. Berul, L. H. Selecting a System for Producing Higher Quality Announcement Journals, Information Dynamics Corp., Wakefield, Mass., 1962. 53 p.

43. Black. W. W. The Data-Phone and Related Means of Transmission. in Research and Engineering Council of the Graphic Arts Industry, Proceedings of the 13th Annual Conference, 1963. pp. 115-120. 
44. Blondeau. P. The IBAI fomputer in the Graphic Arts, in Research and Enginecring Council of the Graphic Arts Industry, Proceedings of the I3th Annual Conference. 1963, pp. 8t-91.

45. Blundell, II. E. Printing Revolution. Research Push Brings Speedier Typesetting, Other Major Advances. The Wall Street Journal $C L X I V, 1,28$ (10 Dec 1964).

46. Bouffilh, R. and C. Savigny. Assignors to Société Anonyme dite: Areliers Bariguand and Marre. Arcuril (Seine), France. Halic Forming Anamorphotic Device for use Photo-Composition. U.S. Patent 2,976,78.5. Patented 28 Mar 1961; filed 18 Sep 1956: (Class 95-4.5).

47. Bouffili, R. and C. Savigny. Assignors to Socićté Anonyme dite: Ateliers Bariquand and Marre, Arcueil (Seine), France. Optical Line-Justification Device for PhotoComposition, U.S. Patent 2,939,370. Patented 7 June 1960; filed 18 Sep 1956; (Class 95-4.5).

48. Boyd, R. Monitor Controlled Computer Processing of Bookwork, in Institute of Printing, Comp. Typesetting Conf Report of Proceedings, 1965, pp. 25-33.

49. Bozman, W. R. Phototypesetting of Computer Output, An Example Using Tabular Data, NBS Tech. Note 170. National Bureau of Standards. Washington. D.C. 25 June 1963, 6 p.

50. Brooke-Wavell, B. New Computer Output Recorders, British Communications Electronics 5, 928-932 (1958).

51. Browne, J. J. Investigation of Typographic Requirements for Mathematical Composition, App. No. 4 in Mergenthaler linotype Co., Report on the Study and Investigation for the Desion of a Lexical.Graphical Composer-Printer System, 1963, 9 p.

52. Browne, J. J. Output Pare Format for the LC;CP System, App. No. 1 in Mergenthaler Linotype Co., Report on the Siudy and Investigation for the Desien of a Lexical(iraphical Composer-Printer System, 1963. 10 p.

53. Browne, J. J. and M. (;urien. Report on an Investigation of a llethod for Automatic Hyphenation Suitable for Computer Application, App. No. 2 in Mergenthaler Linotype Co., Report on the Siudy and Investigation for the Desion of a Lexical-Graphical Composer-Printer System, 1963. $19 \mathrm{p}$.

54. Buck, C. P., R. F. Pray, III and (;. W. Walsh. Investigation and Study of Ciraphic-Semantic Composing Techniques, RADC-TR-61-58. Final Rept. Contract AF 30(602)2091. Syracuse Univ. Research Inst. June 1961, $1 \mathrm{v}$.

55. Buckland, L. F. The Use of a Cathode Ray Tube Display Console for Editin" Textual Information, in H. P. Luhn [ed], Automation and Scientific Communication. Short Papers, Pt. 2, 1963, p. 179-180.

56. Buckland, L. F. Machine Recording of Textual Information during the Publication of Scientific Journals, Inforonies, Inc.. Maynard, Mass., May 30, 1965, 70 p.

57. Buroer, J. B. High Speed Display of Chemical Nomenclature, Molecular Formula and Structural Diagrams. CIDS Rept. No. 4, (ieneral Electric Co., Huntsville, Ala., 31 Dec 1964, 17 p, Contract DA-01-02-AMC-242(Z).

58. Busa, K. Complete Index Verborum of St. Thomas Aq., Speculm, July 1950, p. 424-425.

59. Busa, R. The Index of all non-Biblical Dead Sea Scrolls Published up to December 1957, Revue de Qumran I, 187-198 (1958).

60. Busa, R. Rapidissimo Composizione di Indici e Concordanze de Parole Mediante Schede Perforate, in XVIII Congresso Mondiole della Documenta, Rome, Sep 1951.

61. Cahn, J. N. A System of Information Systems, Paper in L. H. Hattery and E. M. McCormick [eds], Information Retrieval Management, 1963, p. 21-30.

62. Caldwell, S. H. The Sinotype: a Machine for the Composition of Chinese from a Keyboard, J. Franklin Institute 267, 471-502 (1959).

63. Cameras, Cards Create Catalogs, Systems Manarement (April-June 1961).

64. Carroll, J. M. Trends in Computer Input/Output Devices, Electronics 29, 142-149 (Sep 1956).

65. C.BS Laboratories. VIDIAC (;A-1000 Ciraphics Arts Composer Printer. Proposal CLD No. 152. Stamford. Conn.. 5 May 1961. 37 p.
66. CIS Newsletter, I Aug 1965. Composition Information Service, Los Angeles. Calif., issued at various dates. 67. Citron, J., L. Mart and H. OlsIman. A Permutation Index to the "I'reprints of the International Conference on S(cientific Information", Rept. uo. Sl'-44 (rev). System Development Corp., Santa Monica, Calif., 15 Dec 1959, $37 \mathrm{p}$.

68. Clapp, V. W. Research in Problems of Scientific Information-Retrospect and Prospert, Amer. Document d. tion 14, 1-10 (1963).

69. Clapp, V. W. Libraries and the 'Appercase Limitation', in B. E. Markuson [ed], Libraries and Automation. 1964, pp. 54-5.5.

70. Coates, E. J. Bibliographical Indexes, in Institute of Print in:. Comp. Typesenting Conf., Report of Proceedings, 1965, pн. 44-49.

7l. Communication Problems in Biomedical Research, Report of a Study by the Division of Medical Sciences National Academy of Sciences-National Research Council in cooperation with the Federation of American Societies for Experimental Biolowy and with the Institute for Advancement of Hedical Communication. Washimeton, D.C. Oct 31, 1963, 24 p.

72. Composition Information Services. CIS Newsletter, various issues.

73. Composition Information Services, Computerized Type setting. A Management Report on the State of the Art, Los Angeles, Calif,, Oct 1964, 11 p.

74. Compugraphic Corp. The Complexity of Tabular Composi ion and its I limate Simplification, Descriptive Brochure, Compugraphic Corp., Brookline, Mass., n.d., 7 p.

75. Compngraphic Corp. The LINASEC Computer, Descriptive Brochure, Brookline, Mass., n.d., 3 p.

76. Compunaphic Corp. A New Approach to the Composition of Telephone Directory Listings, Descriptive Brochure, Brookline, Mass., n.d., 5 p.

77. Computer Abstracts, Cambridge Communications Corp. Cambridıe, Mass., Vol. 1, 1959/60.

78. Computer-Directed Typesetting System, Reprinted from Printing Production. Jan 1964, 3 p.

79. Computer Hyphenation Experiments Lead to New Thounhts on Typesetting Quality, Book Production Magazine 79, 45 (Mar 1964).

80. Computer Output Printer, Electronic Technology 38 356 (1961).

81. Computer Sets Ads for New Offset Daily, Editor and Publisher 94, 14 (18 Nov 1961).

82. Computer System Will Speed AP's Stock Market Tables. Editor and Publisher 94, 9-10 (1961).

83. Computers Now Speak with Pictures, Systems Management, (Nov 1962) pp. 30-33.

84. Cone, D. R. High Speed Methods for Printing Computer and Tape Data, presented at Symposium in High-Speed Printing of Computer and Tape Data, in TAl;A, Proceedinus, 13th Annual Meeting, 1961. pp. 181-19.4.

85. Conrad. G. M. Biolowical Abstracts Tools up for the Future, AIBS Bulletin 10, 25-27 (Oct 1960).

86. Control Data Corp. Control Data 8080 Computer-Controlled Typeselting System, Descriptive Brochure, Minneapolis, Minn., 1964, 6 p.

87. Cornelius, M. E. Machine Input Problems for Machine Indexing: Alternatives and Practicalities, in American University. Machine Indexing: Progress and Problems, 1962. pp. 41-49.

88. Corliss, C. H. and W. R. Bozman. Experimental Transition Probabilities for Spectral Lines of Severity Elements, NBS Monograph 53, National Bureau of Standards. Washington, D.C., 20 July 1962,562 p.

89. Corrado, V. M. The Linotron, in Research and Engineering Council of the Fraphic Arts Industry, Proceedings of the 14ih Annual Conference, 1964. pp. 109-118.

90. Corrado, V. M. The Integration of Computers and High Speed Typesenting into the Composing Room, in Automatic Typesening, 1965, pp. 271-306.

91. Council on Library Resources Inc. I.ibrary of Conuress Sponsors Experimental Demonstration Involving Use of Tape-Producing Typewriter for Versatile Automated Printing, Recent Developments No. 129. Washinton, D.C., 4 May 1964. 3 \%. 
92. Crockett. R. (.) New Photon Composin $\%$ Machine Will Set Type from the Output of a Computer, in H. P. Luhn [ed]. Automation and Scientific Communication. Short Papers. Pt. 2. 1963. pp. 317-318.

93. Crosfield Electronics, Inc. Automate Your Composing Room with Roboset Tape Perforators and Linecasting Machine Operating Lnits. Descriptive Brochure. Westbury, N.Y., n.d., 6 P.

94. Croxton, F. E. and W. J. Wilson. Order in the Court of Mixed Notations, Data Proc. Magazine 7,31 (July 1965).

95. Davie, II.A. J. High-Speed Printers, Inst. Radio Engineers $20,675-683$ (1960)

96. Day. II. S. and I. Lebow. New Indexing Patterns for Nuclear Science Abstracts. Amer. Documentation 9. 120-127 (1960).

97. Dewitt, F. Typesetting Takes Too Much Time. Printing Production 91, 41-45, 108, 109 (Feb 1961).

98. Dick. (A. B.) Co. Lexical-Graphical Composer Printer for Rome Air Development Center. Griffiss AFB, N.Y. Proposal No. P61-102. Chicaro. 11l.. 20 Oct 1961, 19 p.

99. Dick. (A. B.) Co. The L se of Videograph Techniques for (iraphic/Semantic Composition in the Baird AtonicIBU Subsystem of Machine Translation. Proposal, Chicaso. Ill. 10 Aue 1960. 18 p.

100. Dick. (A. B.) Co. Videograph Computer Printer for Vational I.ibrary of Medicine MEDLARS Program. Technical Proposal. Vo. P6I-124. Chica:o. Ill.. 1961, 26 p.

101. Documentation, Inc. Final Report to the Division of Tech nical Information, U.S. Atomic Energy Commission on Contract Jo. AT (49-12)-1867, W ashington, D.C., 1961.

102. Duncan, C. J.. in Computer Typesettin!- Conference Pro ceedintrs 1965. Computer Typesettini-A lew Boom? in Automatic Typesettin:. 1965. pp. 39-56.

103. Duncan. C. J. Computer Typesetting Research at the Lniversity of Newcastle-Lpon-Tyne, Composition Information Services. Los An⿺eles, Calif., 13 July 1965. 2 v.

104. Duncan, C. J. Look! No Hands!. in H. Spencer [ed]. The l'enrose Annual, 1961. pp. 121-167.

105. Duncan. C. J. Proposed System of Type Composition. l'rinting Technolo=y 5. 77-86 (Nov 1961).

106. Duncan. C. J., J. Eve, L. Molyneux, E. S. Paqe and II. (;. Robson. Computer Typesettinu: An Evaluation of the Problems, Printing Technology $7,133-151$ (Dec 1963).

107. Dura Business Machines, Inc. Dura Converters for High Speed Courdination of all Machine lan-uawe Codes. Descriptive Brochure. Madison Heiwhts. Mich.. 1962. 2 p.

108. Dura Business Vachines. Inc. Dura Mach 10-Automatic Typing Most Advanced Achievement, Descriptive Brochure, Madison Heirhts. Mich.. 1962, 6 p.

109. Dyson, M. (; and M. F. I.ynch. A Computer-Produced Express Dimest, Chemical Abstracts Service. The Ohio State University, Columbus. Mimeouraph. n.d.. 28 p.

110. Dyson, .I. (;. Current Research at Chemical Abstracts. J. Chem. Documentation I, 24-28 (1961).

111. Epstein. H. The Electrowraphic Recording Technique. in (American Federation of Information Processing Sucieties), l'roceedinurs of the Western Joint Computer Conference, 195.5, pp. 116-118.

112. Elstein, H. and P. Kintner. The Burrou»hs Electrowraphic Printer-Plotter for Ordnance Computin.. in (American Federation of Information Processine Societies). Proceedings of the Eastern Joint Computer Conference. 1956, pp. $73-80$.

113. Fairchild (;raphic Equilument Corp. Fairchild TTS Cniversal Perforator. Descriptive Brochure, l'lainview. 1.. I.. X. Y.. n.d.. 2 p.

114. Fairchild Graphic Equipment Corp. A Key to Increased Composin: Room Profits: More Tyue in Less Time Through Automatic Typecasting, Descriptive Brochure. Plainview, L. I.. I. Y.. n.d., 23 p.

115. Fairchild (;raphic Equipment Corp. Teletypesetter Perforator Operator's Training Reference Manual, Plainview, I.. I., \. Y.. n.d.. 74 p.

116. Fairchild Craphic Equipment Corp. The Teletypesetter System. Descriptive Brochure, Plainview, L. I., N. Y.. n.d.. 4 p.
117. Fasana. P. An Approach to the Mechanization of a Syndetically Integrated Subject Heading Authority File, Special Rept. No. 4. Contract AF 19(604)-8438, ltek Corp.. Lexinuton. Mass., 1963.

118. Feldman, A. A Proposed Improvement in the Printing of Chemical Structures, Which Results in Their Complete Computer Codes, Amer. Documentation 15, 205-209 (1964).

119. Filmsettin $\%$ and Cold Type Methods. A 'Print in Britain Checklist of Machines. Materials and Methods. Print in Britain supplement, Nov 1964, 16 p.

120. Flint, C. M. The Next Step, in Research and Engineering Council of the Graphic Arts Industry. Proceedings of the 13th Annual Conference, 1963, p. 125-127.

121. Flynn, G. A Practical Apulication of Facsimile, in Research and Engineering Council of the Graphic Arts Industry. Proceedings of the 13 th Annual Conference. 1963, p. 108-115.

122. Freeman, R. R. and M. G. Dyson. Development and Production of Chemical Titles, A Current Awareness Index Publication Prepared with the Aid of a Computer, J. of Chem. Documentation 3, 16-20 (1963).

123. Friden, Inc. Compos-o-line Sequential Card Camera, Descriptive Brochure, Rochester, N.Y.. n.d.. 6 p.

124. Friden, Inc. Friden Compos-o-line, Manual of Instructions, Rochester, N.Y., 1961, 23 p.

125. Friden, Inc. Justowriter, Descriptive Brochure, Rochester. N.Y.. n.d.. 6 p.

126. Friden. Inc. Press Mire Justowriter: Automatic TapeOperated Copysetting Machine. Descriptive Brochure. Rochester, N.Y.. n.d.. 11 p.

127. Friden, Inc. Punched Tape Code Converter, Technical Wirin Manual. Rochester, V.Y., 1961, 50 p.

128. Friden, Inc. Technical Manual for Friden Flexowriter Programatic. Rochester, X.Y.. 1962, 49 p.

129. Friden. Inc. Technical Manual for Friden LCC-S Justowriter, Rochester. N.Y., 1961, 37 p.

130. Fullick, R. F. Problems of a Book Printer, in Institute of Printing, Comp. Typesetting Conf.. Report of Proceedings, 1965 , p. 22-24.

131. Fulton, R. L. Visual Input to Computers, Datamation 9, $37-40$ (Aug 1963).

132. Calli. E. J. The Stenowriter: A System for the Lexical Processing of Stenotypy, IRE Trans. Electronic Com. puters EC-11, 187-199 (1962).

133. ('ardner. A. E. Computers and Typesetting. The Graphic Arts Monthly 34, 48-53 (June 1962).

134. (,arfield. E. I L'nified Index to Science. in National Academy of Sciences-National Research Council. I'roceedings of the International Conference on Scientific Information. Vol. I. 1959. v. 461-474.

135. (.arfield. E. Can Citation Indexing Be Automated?. in |'roceediners of the Symposium on Statistical Association Methods for Mechanized Documentation. NBS Misc. Publication 269, Government l'rintin: Office, II ashinet on. D.C... publication pendin.

136. (;arfield. E. Citation Indexes for Science, Science 13., 108-111 (15 July 19.55).

137. Carfield. E. The Preparation of Printed Indexes by Automatic Punched-Card Equipment: A Manual of Procedures. Welch Medical Library, Johns Hopkins Lniv.. Baltimore, Md.. 24 Mar 1953, 16 \%.

138. (; arfield. E. The Preparation of Printed Indexes by Automatic Punched Card Techniques. Amer. Documentation 6. 68-76 (1955)

139. (iarth, W. W. Jr., assignor to (iraphic Arts Research Foundation. Inc. Cambridue Mass. Type Composinw Apparatus. L.S. Patent 2.888.865. Patented 2 June 1959. filed 16 Viar 1956. (Class 95-4.5).

140. Cieneral Dynamics/Electronics. Computer Recorders (S-C 4020). Descriptive Brochure, San Dieøo. Calif. 1961,4 p.

141. (ieneral Dynamics/Electronics. S-C 4020 System Description. Descriptive Material. San Dierro. Calif., n.d.. 13 p.

142. 1.eneral Electric Co. MEDLARS Preliminary Design, Phase 1. Final Technical Rept. to the National Library of Medicine on Contract I'H 86-62-8, W ashinston. D.C. 1962. 143 p. 
143. (,iallanza, F. V. and J. H. Kennedy, Keyword-in-Title (KWIT) Index for Reports, Rept. No. I/CRL-6782, L.awrence Radiation Lab., I niv. of California, Livermore, 14 May $1962,8 \mathrm{p}$.

144. Coldlor, Il. [ed]. P'rocecdings of the 1963 Clinic on Library Applications of Data P'rocessing, Craduate School of library Science, Lniversity of Illinois, Champaien, IIl., $1964,176 p$.

145. Coodwin, P. B. Composinz Room Computer, Data Proc. for Management 6, 34 (Mar 1964).

146. Ciraphic Arts Index, in Graphic Arts Progress. (iraphic Arts Information Service, Rochester Institute of Tech. nology, Rochester, N.Y.

147. Cray, B. S. Automatic Typing of literature Searches, J. Chem. Doc. I, 71-73 (1961).

148. Crea, R. A., E. P. Hanson, R. A. Higonnet and L. M. Moyroud. Type Composin" Apparatus, U.S. Patent 2,988,276. Patented 13 June 1961; filed 7 Jan 1954 (Class 235-151).

1.49. (ireen, F. V. Photowraphy Speeds Directory Printing, reprint from Inland P'rinter/American Lithowrapher, Aur 1961, 2 p.

150. Griffin, M. The Library of Tomorrow, Technical Memorandum ASDJ-M-040, International Business Machines Corp., San Jose, Calif., 1961, 17 p.

151. Grove, J. F. Photocomposition in Book Publishing, Book Production 7\%, 48-56 (Nov 1960).

152. Gull, C. D. Automatic Documentation, Current Systems and Trends in the USA, Rev. Int. Doc. 29, 57-62 (1962): also in Guidelines to Mechanizing Information Systems, in L. H. Hattery and E. M. McCormick [eds], Information Retrieval Management, 1962, pp. 101-110.

153. Hagan, W. C. Kingsport and Computers, Book Production Magazine 79, 46-47 (Mar 1964).

154. Harris-Intertype Corp. Harris-Intertype Demonstrates Electronic Typesetting System, Production News 1, No. 1 (7-13 June 1964), $4 \mathrm{p}$.

155. Harrison, V. G. W. Co-operative Research in the Graphic Arts Industries, in 14. Spencer [ed], The Penrose Annual, 1964, p. $1-8$

156. Hattery, L. H. and E. M. McCormick [eds], Information Retrieval Management, (Papers adopted from Proceed. ings of the American University Fourth Institute on Information Storage and Retrieval 1962), American Data Processing, Inc., Detroit, Mich., 1963, $151 \mathrm{p}$.

157. Hell, R. Digiset, Composition Information Services, Los Angeles, Calif., n.d., 6 p.

158. Herner, M. and B. Kelber. Speed of Announcement 0 . United States Government Research Reports in Technica? Abstract Bulletin, U.S. Government Research Reports, and Nuclear Science Abstracts, 1961-62, Report to the National Science Foundation on Contract NSF-C213, Herner and Co., Washington, D.C., 1962, 13 p.

159. Herner, S. and M. Herner. Factors Governing the Publication of United States Government Research Reports, Report to the National Science Foundation, Herner and Co., Washington, D.C., 1962, $72 \mathrm{p}$.

160. Heynick, L. N., R. J. Wohl and D. H. Andrews. Permanent Writing, Cathode-Ray Recorder, Electronics 29 148-149 (Apr 1956).

161. Hider, T. M., J. J. Rennilson and R. A. Frech, assignors to General Dynamics Corp., Rochester, N.Y., Electronic Printer, U.S. Patent 3,047,871. Patented 31 July 1962; filed 31 Mar 1958, (Class 346-110).

162. Higonnet, R and L. Moyroud, assignors to Graphic Arts Research Foundation, Inc., Cambridge, Mass., Photo Composing Machine, U.S. Patent 2,790,362. Patented 30 Apr 1957; filed 23 Aug 1947, (Class 95-4.5).

163. Higonnet, R. and L. Moyroud, assignors to Graphic Arts Research Foundation, Inc., Cambridge, Mass., Type Composing Apparatus, U.S. Patent 2,951, 428 . Patented 6 Sep 1960; filed 22 Aug 1957, (Class 95-4.5).

164. Hintz, H. P., J. F. Hayden and G. W. Offensend, assiønors to Eastman Kodak Co., Rochester, N.Y., Strip Material Advancing Mechanism, U.S. Patent 2,738,704. Patented 20 Vlar 1956; filed 19 Dec 1952, (Class 88-24).

165. Hadky, W. The Anatomy of an Electronic Typer, Proc. Radio Club of America 37 (Fall 1961) 15 p.
166. Hoffman, R. F. A Survey of Composing Room Materials and Machines, in International Association of l'rintiny House Craftsmen, Printing Progress: A Mid-Century Report, 1959, p. 169-199.

167. Hoisington, E. Report of Task Force ABLE. Agricultural Biological Literature Exploitation, Department of Agriculture, Washington, D.C., Nar $1965,477 \mathrm{p}$.

168. Holm, B. E. Techniques and Trends in Effective Utilization of Enyineering Information, ASI.IB Proc. 17, 134-169 (1965).

169. Hosken, J. C. Survey of Mechanical Printers, in (American Federation of Information I'rocessing Societies), Revicw of Input and Output Equipment Used in Computing Systems, Joint AIEE-IRE-ACM Computer Conference 1952, p. 106-112.

170. How to Slash Composition Costs, Systems 6, 18-19 (Nov 1965).

171. Humphrey, H. H. Aqricultural Research Information and Communication: A Prowress Report, Memorandum from Senator H. H. Humphrey, Chairman, Subcommittee on Reorganization and International Organizations (April 3, 1962), $58 \mathrm{p}$.

172. Information Dynamics Corporation (Formerly Forbes \& Waite). A Compilation of Data on Computer-Output Printers Proposed for MEDLARS, Technical Report to the National Library of Medicine. Information Dynamics Corp., Wakefield, Mass., 1961, 14 p.

173. Institute of Printing, Computer Typesetting Conference, Report of Proceedings, (London University, July 1964), London, Engrand, 1965, 245 p.

174. International Association of Printing House Craftsmen, Inc., Printiny Progress: A Mid-Century Report, Cincinnati, Ohio, 1959, $543 \mathrm{p}$.

175. International Business Machines Corp. Graphic Composing Techniques, Final Rept. on Contract AF 30(602)2527, RADC-TR-61-310, Thomas J. Watson Research Center, Yorktown Heights, N.Y., Feb 1962, 67 p.

176. International Business Machines Corp. Highlights of the IBM Type Composition Proyram. 'Set . . . to 6, White Plains, N.Y., n.d., 8 p.

177. International Business Machines Corp. IBM 63 CardControlled Tape Punch, Manual of Operation, New York, 1958, 126 p.

178. International Business Machines Corp., IBM 870 Document Writing System, Reference Manual, New York, 1961, 84 p.

179. International Business Machines Corp., IBM 1403 Printer: Original Equipment Manufacturer's Information, Reference Manual, New York, 1961, 16 p.

180. International Business Machines Corp., IBM 1404 Printer, IBM 1401 Data Processing Bulletin (1961), 15 p.

181. International Business Machines Corp., IBM 7765 Paper Tape to Magnetic Tape Converter, Reference Manual. New York, 1961, 29 p.

182. Intertype Co., Intertype Handbook of Tape Operation, Descriptive Brochure, Brooklyn, N.Y., 1961, 106 p.

183. Intertype Co., Intertype Linecasting Machines, Descriptive Brochure, Brooklyn, N.Y., n.d., 79 p.

184. Intertype Co., Intertype Monarch, Descriptive Brochure, Brooklyn, N.Y., n.d., 12 p.

185. Intertype Co., Letter Perfect Photocomposition by Fotosetter, Descriptive Brochure, Brooklym, N.Y., n.d., 24 p.

186. Invac Corp., Photoelectric Keyboard, Model PK-144, Descriptive Brochure, Waltham, Mass., n.d., 9 p.

187. Invac Corp., Typewriter-Transmitter/Receiver Model TTR-100, Descriptive Brochure, Waltham, Mass., n.d., $10 \mathrm{p}$.

188. Invac Corp., Typewriter-Transmitter/Receiver Model TTR-200, Descriptive Brochure, Waltham, Mass., n.d., $10 \mathrm{p}$.

189. Janaske, P. [ed.]. Information Handling and Science Information: A Selected Bibliography 1957-1961, American Institute of the Biological Sciences, Washington, D.C., $1962,1 \mathrm{v}$.

190. Jeffree, J. H., assignor to CAPS, Ltd., London, England, Optical Justifying Means, U.S. P’atent 3,051,051. Patented 28 Aug. 1962; filed 7 July 1959 (Class 88-57).

191. Johnston. J. MI. Computers in the Production of TimeTables, in Institute of Printing, Comp. Typesetting Conference, Report of Proceedings, 1965, pp. 49-53. 
192. Kehl, W. B. Computers and Literature, Data Processing Magazine 7, 24-26 (July 1965).

193. Kennedy, J. H. System of Automatic Processing and Indexing of Reports, Rept. No. UCRL-6510, Lawrence Radiation Lab., Univ. of California, Livermore, 12 July 1961, 29 p.

194. King, ( $;$. W. [ed.]. Automation and the Library of Congress (a survey sponsored by the Council on Library Resources, Inc.), Library of Congress, Washington, D.C., 1963, 88 p. Also in The Automation of Library Systems, in B. E. Markuson [ed.], Libraries and Automation, 1964, pp. 233-244.

195. King, P. F., F. Schwertz, G. Mott, R. Rogers, B. Norton, and E. Woodhull, Air Force Teleprinter Project, Final Rept. on Contract AF 33(600)-32251, Haloid Xerox, Inc., Rochester, N.Y., 1958, 21 p.

196. Konigsberg, J. Detailed Analysis of Steps Required to Produce a High Quality Page of Printed Copy Using Conventional Techniques, Methods, and Equipments, App. No. 6 in Mergenthaler Linotype Co., Report on the Study and Investigation for the Design of a Lexical-Graphical Composer-Printer System, 1963, 15 p.

197. Kraft, F. R. Investigation of Optical-Mechanical Projection Approach to the Processing of Graphical and Mathematical Items in the LGCP System, App. No. 5 in Mergenthaler Linotype Co., Report on the Study and Investigation for the Design of a Lexical-Graphical Composer-Printer System, 1963, $17 \mathrm{p}$.

198. Kraft, F. R. The Reduction in Bulk Resulting from the Typesetting of Documents in Modern Composition Systems, in H. P. Luhn [ed], Automation and Scientific Communication, Short Papers, Pt. 2, 1963, pp. 275-276.

199. Kreithen, A. K. The Role of the Computer in the Scientific and Technical Information Facility of the National Aeronautics and Space Administration, Paper Presented at the Symposium on Office Information Retrieval sponsored by the General Services Administration, National Archives and Records Service, 25 Oct. 1962, 11 p. Documentation, Inc., Washington, D.C.

200. Kuney, J. H. Analysis of the Role of the Computer in the Reproduction and Distribution of Scientific Papers, in H. P. Luhn [ed], Automation and Scientific Communication, Short Papers, Pt. 2, 1963, pp. 249-250.

201. Kuney, J. H., R. H. Belknap and B. G. Lazorchak. Progress in Photocomposition, J. Chem. Doc. 1, 44-45 (1961).

202. Kuney, J. H. and B. G. Lazorchak. Machine-Set Chemical Structures, in American Documentation Institute, Parameters of Information Science, 1964, pp. 303-305.

203. Kunkel, G. E. and T. H. Marcum. Hyphenless Justification, Datamation 11, 42-44 (Apr. 1965).

204. Landwehr, J. B., C. McLaughlin, H. Mueller, M. Lichstein and S. V. Pollack. BLNSYS-A 1401 Operating System with Braille Capabilities, Comm. Assoc. Computing Machinery 8, 300-303 (1965).

205. Larkey, S. V. Cooperative Information Processing-Prospectus, Medicine, in J. H. Shera, et al., Documentation in Action, 1956, pp. 301-306.

206. Larkey, S. V. The Army Medical Library Research Project at the Welch Medical Library, Bull. Med. Lib. Assoc. 37, 121-124. (1949).

207. LeBrun, T. Q. An Electronic Retina Optical Character Reading System with Integral Logical Editing and Indexing Capabilities, in H. P. Luhn [ed.], Automation and Scientific Communication, Short Papers, Pt. 2, 1963 , pp. $149-150$.

208. Ledley, R. S. and J. B. Wilson. Investigation of the Use of Digital Electronic Computers in the Publication of the Index Medicus, Final Rept. to the National Library of Medicine on Contract SAph 71251, George Washington Univ., Washington, D.C., n.d., 1 v.

209. Lipetz, Ben-Ami. A Successful Application of Punched Cards in Subject Indexing, Amer. Documentation 11 , 241-246 (1960).

210. Lipetz. Ben-Ami. Compilation of an Experimental Citation Index from Scientific Literature, Technical Rept. IL4000-19, Itek Corp., Lexinuton, Mass., 1961, 25 p.

211. Lipetz, Ben-Ami. Labor Costs, Conversion Costs, and Compatibility in Document Control Systems, Amer. Document atioñ $14,117-122$ (1963).
212. Lombardi, L. A. Machine Translation and Automation of the Printing Process, J. Mach. Accounting 14, 22-26 (Jan. 1963).

213. Luce, D. A., and M. P. Barnett. Practical Operating Experience with a Tape Controlled Photon S-560 Unit, in H. P. Luhn [ed.], Automation and Scientific Communication, Short Papers, Pt. 2, 1963, pp. 293-294.

214. Luhn, H. P. [ed.]. Automation and Scieutific Communication, Short Papers, P't. 1, American Documentation lnstitute, Washingt on, D.C., 1963, pp. 1-128.

215. Lulı, H. P. [ed.]. Automation and Scientific Communication, Short Papers, P't. 2, American Documentation Institute, Washington, D.C., 1963, pp. 129-384.

216. Luhn, H. P. Keyword-In-Context for Technical Literature (KWIC Index), presented at American Chemical Society Division of Chemical Literature at Atlantic City, N.J., 14 Sep. 1959. Rept. No. RC-127, International Business Machines Corp., Yorktown Heiglits, N.Y., 1959, 16 p. Also in Amer. Documentation 11, 288-295 (1960).

217. Luhn, H. P. The Automatic Creation of Literature Abstracts, IBM J. Research and Development 2, 159-165 (1958).

218. Makris, C. J. Survey and Investigation of Character Generators and Display Technique Methods, App. No. 9 in Mergenthaler Linotype Co., Report on the Study and Investigation for the Design of a Lexical-Graphical Composer-Printer System, 1963, 54 p.

219. Markus, J. Computer Experience: Directory Production, Mc(iraw-Hill, Inc., New York. Paper presented at the Institute on Electronics and Automation in Publishing, American University, May 11, 1965, 13 p.

220. Markus, J. Computers in Commercial Publishing, McGrawHill, Inc., New York, 15 Mar 1965, 15 p.

221. Markus, J. State of the Art of Published Indexes, Amer. Documentation 13, 15-30 (1962).

222. Markuson, B. E. [ed]. Libraries and Automation, (Proceedings of the Conference held at Airlie Foundation, Warrenton, Va., 26-30 May 1963, under sponsorship of the Library of Congress, the National Science Foundation, and the Council on Library Resources), Library of Congress, Washington, D.C., 1964, 268 p.

223. Mayo, C. R. Electrostatic Printing Today and Tomorrow, in Research and Engineering Council of the Graphic Arts Industry, Proceedings of the 14th Annual Conference, 1964, pp. 74-79.

224. Mergenthaler Linotype Co. The Diagrammer: a New Precision Tool for Mechanized Production of Symbolic Diagrams, Descriptive Brochure, Brooklyn, N.Y., n.d., 7 p.

225. Mergenthaler Linotype Co. The Linofilm Converter, Descriptive Brochure, Brooklyn, N.Y., n.d., 6 p.

226. Mergenthaler Linotype Co. The Linofilm System: a New Concept in Photocomposition, Descriptive Brochure, Brooklyn, N.Y.

227. Mergenthaler Linotype Co. Linomatic Tape System Operating Unit, Descriptive Brochure, Brooklyn, N.Y., n.d., 4 p.

228. Merwenthaler Linotype Co. Linomatic Tape System Perforator, Descriptive Brochure, Brooklyn, N.Y., n.d., 4 p.

229. Mergenthaler linotype Co. The Linotype Line, Descriptive Brochure, Brooklyn, N.Y., n.d., 22 p.

230. Mergenthaler Linotype Co. Mergenthaler News for Release, Brooklyn, N.Y., 1962, 2 p.

231. Mergenthaler Linotype Co. The Modern and Profitable Make-up Department: Linofilm System Composer, Descriptive Brochure, Brooklyn, N.Y., n.d., 4 p.

232. Mergenthaler Linotype Co. The New Linotype Elektron, Technical Bulletin, Brooklyn, N.Y., 1962, 8 p.

233. Mergenthaler Linotype Co. A Short Introduction to the Composition of Display Mathematical Formulas at the Linofilm Keyboard, Descriptive Brochure, Brooklyn, N.Y., n.d., 10 p.

234. Mergenthaler Linotype Co. The Linotron, Brooklyn, N.Y., n.d., $3 \mathrm{p}$.

235. Mergenthaler Linotype Co. This is Iinofilm, Brooklyn, N.Y., n.d., 12 p.

236. Mergenthaler Linotype Co. Report on the Study and Investigation for the Desion of a Lexical-(iraphical Composer-Printer System, RADC-TDR-63-63, Final rept. 
on Coutract If 30160212624 , Brooklyn, \. ).,31 Jan. 1963. I $\mathrm{v}$

237. Mer\%, M. J., Jr. Optical Character P'age Readers, in Re. scarch and Enpinecrin: Council of the Ciraphice Arts

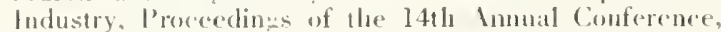
$1964,[$ ए. $8 \mathrm{I}-85$.

238. Vikhaitov, A. I. Problems of Mechanization and Auto. mation of Information Work, Rev. Int. Doc. 29, 49-56 (1962).

239. Mills, J. The I niversal Decimal Classification, Vol. I of the Rutwers Series on Systems for the Intellectual Oranization of Information. S. Artandi [ed.], Ciraduate Sohool of I.ibrary Service, Rutwers University, New Brunswick, N.J.. 1964, 132 p.

240. Monotype Composition Caster, Engineering 192, 850-852 (29) Dec, 1961).

241. The Ilonotype Corp. Vonophoto Filmsetters Simply Explained, Descriptive Brochure, Philadelphia, l'a.. n.d.

242. Mooers. ( $:$ N. The Tale Typewriter I'lan: a Method for Cooperation in Documentation, ASLIB Proc. 12, 277-291 (1960).

243. Moore, J. K., and M. Konenbery. f, enerating HighQuality Characters and Symbols, Electronics 3.3, 5.5-59 (10) June 1960).

244. Worton, C. The Linasec, in Researeh and Eneinerring Council of the firaphic Arts Industry, Proceedinus of the 13th Anmual Conference, 1963, pp. 95-101.

245. Moyroud, 1. Zip, in Research and Enpineerin: Council of the firaphic Arts Industry. I'roceedings of the $141 \mathrm{~h}$ Annual Conference, 1961, p). 106-109.

246. Muloek, I. E., and M. I'. Albert, assignors to International Business Machines Corp., New York. Cathode Ray Tube Printer, U.S. Patent 3,041,947. Patented 3 July 1962; filed 16 Nov, 1959 (Class 95-4.5).

247. McNaney, J. T. Electron Gun Operates High Speed Printer, Electronics 31, 74-77 (26 Sep 1958).

248. National Academy of Sciences-National Research Council. Proceedings of the International Conference on Scientific Information, Washington, D.C., 1959, Vol. I. pl. 1-813; Vol. II. pp. 817-1635.

249. National Library of Medicine, Index Megdicus, Vol. 5, No. 8, Aus. 1964, 1 v.

250. National Library of Medicine, The MEDLARS Story at the National Library of Medicine, Washington. D.C.. Covernment Printin: Office, 1963, 74 p.

251. National Library of Merlicine, The National I.ibrary of Medicine Index Mechanization l'roject, Bull. Med. Lib. Assoc. 49, 1-96 (1961).

252. National Science Foundation, Current Research and De velopment in Scientific Documentation, No. 7 (NSF-6065), Nov 1960, 153 p.; No. 9 (NSF-61-76), Nov 1961, 270 p.; No. 10 (NSF-62-20), May 1962, 383 p., Government Printing Office, Washington, D.C.

253. New Computer Prouram Automatically Prepares Data and Produces Offset Printing Plates for Publication. NBS Standard 7 (Sep. 1962).

254. O'Connor, J. Some Remarks on Mechanized Indexing and Some Small-Scale Empirical Results, in American Iniversity, Machine Indexing: l'rogress and P'roblems, 1962, pp. 266-279.

255. O'DonnelI, B. 1. The Relative Costs of Letterpress and Typewriter Settine. J. Documentation 17, 150-155 (1961)

256. Oettinger, 1. (,., W . Foust, V. (,iuliano, K. Magassy and L. Matejka. Linguistic and Machine Methods for Compiling and Updating the Harvard Automatic Dictionary, in Proceedings of the International Conference on Scientific Information, Vol. 11, 1959, pp. 951-973. National Academy of Sciences - National Research Council.

257. Office Copying - A Technology in Flux, A C \& EN Special Report, Chemical and Engineering News 42, 114-129 (13 July 1964); 42, 84-96 (20 July 1964).

258. OhIman, H. State-of-the-Art: Remote Interrogation of Stored Documentary Material, in H. P. Luhn [ed.|, Automation and Scientific Communication, Short Papers, Pt. 2, 1963, pp. 193-194.

259. Ohtman, H. Pro a Sperial IR Lanquaze, Comm. Assoc. Computin: Machinery 5. 8-10 (1962).

260. Ohringer, I.. Accumulation of Natural Lanwuge Text for Computer Manizulation. in American Documentation
Institute, Parameters of Luformation Science, 1964. pp. $311-313$.

261. Orehard-Hays, W. General Data Files and Processing Operations, General Information Manual, IBM Conference on Information Retrieval, Sep 1959. International Business Mar.hines Corp., New York, N.Y., 1959.

262. Orton, F. E. A I nified Subject Index 10 Science I.iterature, Amer. Documentation 12, 32-34 (1961).

263. Patrick, R. L., and D. V. Black. Index Piles: Their Loadine and Organization for ['se, in B. E. Marhuson [ed.], L, braries and Automation, 1964, p1. 29-53.

264. Pay, R. G. Typesetting by Computer, Data Processing 5 , 326-333 (1963).

265. The P'enrose Survey, in H. Spencer [ed.], The Penrose Annual, 1964, pp. 289-300.

266. Photocomposition by Computer Program, NBS Technical News Bulletin 46, 145-146 (1962).

267. Photon Corp. Introducin̈ a New Era of I'eah Composine Room Efficiency: I'hoton Series 540, Descriptive Brochure, Wilminuton. Mass., n.d., 4 p.

268. Photon Corp. Photon Composition, Descriptive Brochure, Wilmington, Mass., n.d., $17 \mathrm{p}$.

269. Photon Corp. Whoton Zip-Model 900 Hiıh Speed Com. puter l'hoto-Typesetter, P'roduet Report, Wilmin„ton. Mass., n.d., 12 \%.

270. P'hoton Corp. I'hoton 200-New Dimensions in Photo. composition, Wilmineton, Mass., n.d., 18 p.

271. Photon Corp. The ZIP, a Hiah Speed l'rint-Out Device, Descriptive Brochure, Wilmington, Mass., n.d., 2 p.

272. I'hototypesetting l'ichs L p' the l'are, Business Weeh. MI. 156-158(14 Dec. 1963).

273. I'ittsburwh Lniv. Development of Chinese lileographic Composing Machine, Rept. on Contract DA 36-039AWC-3785(x). l'ittsbureh, l'a.. July 1964, 12 p.

274. Potter Instrument Co.. Inc. Potter Model L.P-600 Hi: hSpeed I'rinter, Descriztive Brochure, I'lainview, L. I., N.Y., n.d.. 2 p.

275. I'resident's Science Advisory Committee, Science, t;overnment, and Information (the Weinbers Report), Washinston, D.C., Government I'rinting Office, 10 Jan. 1963, $52 \mathrm{p}$

276. Price, W. H. A Computer Oriented Photo-Composition System for STAR, in H. P'. Iuhn [ed.], Iutomation and Scientific Communication Short Papers, P'. 2. 1963 , pp. 261-262.

277. Printing lbstracts, v. 1-1945. P'rinting, Packaging and Allied Trades Association, Potra House, leatherhead. Surrey, Enpland, 1945.

278. I'rintin' Breakthrounh Demonstrated. The Palm Beach Post-Times LIV, 1 (14 Jan 1963).

279. Prints Mailing Labels at Computer Speed, Product Engjneering 32, 76-79 (20 Feb. 1961).

280. Proceedings of the Librarians' Convention Helal in New York City, September 15, 16, and 17, 1853, Reprinted for William H. Murray, The Torch Press, Cedar Rapids, Iowa, 1915, $63 \mathrm{p}$.

281. Productivity up $50 \%$ in Cold-Type Ad Slıop, Editor and Publisher, (Mar. 26, 1960) p. 15.

282. Programme de Justification de Textes, Electro Calcul. 6, 51-53 (Nov-Dec 1964).

283. Punching vs. Recording of Paper Tape, Automatic Control 15, 41-43 (Dec. 1961)

284. Radio Corp. of America, Chinese Ideographic I'hotocomposer, (Developed by RCA under contract to U.S. Army Natick Research Laboratories), Descriptive Brochure, Camden, N.J., n.d., 4 p.

285. Radio Corp. of America. RCA 301 Computer for Newspapers, Descriptive Brochure, Camden, V.J., n.t., 6 p.

286. Recker, G. Systen Speeds Cold-Type Composition, The Graphic Arts Monthly 32, 8-10 (June 1960).

287. Recordak Corp. The Recordak Listomatic Camera-Model 3, Descriptive Brochure, Rochester, N.Y.. 1962, 6 p.

288. Recordah Corp. Recordah Listomatic Film Processor, Rochester, N.Y., 1959, l p.

289. Reed, J. B. Some Impediments to Co-operation in Translations: Copyright and Other Matters, ASLIB Proc. 12, 158-166 (1960). Conference on Technical Translation. London, 15 Jan. 1960. 
290. Reitz, G. The Use of Consoles in Machine Translation, in American Documentation Institute, Parameters of Information Science, 1964, p. 277-283.

291. Research and Enqineering Council of the Graphic Arts Industry, Inc. Proceedings of the 13th Annual Conference, (Chicago. Ill., 20-22. Nay 1963). Washington, D.C., 1963. 132 p.

292. Research and Engineering Council of the Graphic Arts Industry, Inc. Proceedings of the 14th Annual Conference, (Rochester, N.Y., 18-20 May 1964), Washington, D.C., 1964, 125 p.

293. Resnick, A. The "Information Explosion" and the User's Need for Hard Copy, in American Documentation Institute. Parameters of Information Science. 1964, p. 315317.

294. The (R)evolution in Book Composition, Special Report. Part I, Computers Are Here-What Now? Book Production Mag. 79, 54-60 (Feb 1964); Part II, Computers in '64. Year of Transition from Theory to Practice, 79, 44-50 (Mar 1964); Part III, What's Ahead for Computers, 79, 55-61 (Apr 1964); Part IV, The Systems Concept Key to Computer Profits, 79, 67-73 (May 1964).

295. Rhoads, W. L. Summaries of the TAGA Papers, Gravure, (Oct. 1961), pp. 20-24: Part 2 (Nov. 1961), pp. 20-21, 100-101; Part 3 (Dec 1961), pp. 24, 26. 29, 32 .

296. Rich. R. P., and A. G. Stone. Method for Hyphenating at the End of a Printed Line, Comm. Assoc. Computing Machinery 8, 444-445 (1965).

297. Richter. D. How Many More New Journals? Nature 186, 18-19(1960).

298. Rogers. F. B. Output Printing: Introductory Remarks. in B. E. Mlarkuson [ed.], Libraries and Automation. 1964. pp. 191-193.

299. Rogers, F. B., and S. Adams. The Army Medical Library's Publication Program, Texas Reports on Biolory and Medicine 8, 271-300 (1950).

300. Rossetto. L., 6. F. Squassoni. E. F. Coleman, and W. S. Gorrill, assignors to Mergenthaler Linotype Co., New York. Photocomposing ITachine, U.S. Patent 2,847,919. Patented 19 Aug. 1958; filed 13 Mar. 1953 (Class 95-4.5).

301. Rossheim, R. J. Nonmechanical High-Speed Printers (in American Federation of Information Processing Societies), Review of Input and Output Equipment Used in Computing Systems, Joint AIEE-IRE-ACM Computer Conference 1952, pp. 113-117.

302. Savage, T. R. The Preparation of Auto-Abstracts on the IB.I 704 Data Processing System, IB.I Research Center, Yorktown Heights. N.Y., 17 Nov, 1958, 11 p.

303. Schmidt, E. I. ASDD and Research Library Procedure Ianual, Special Rept., International Business Machines Corp.. San Jose, Calif., 1962. 45 p.

304. Seehof, J.. II. Armstrong, G. Farley. M. Leinberger. MI. Markakis, and S. Smithbery. The National Cash Rey. ister High Speed Marnetic Printer (in American Federation of Information Processing Societies), Proceedings of the Eastern Joint Computer Conference 1957, Washinton, D.C., Dec. 9-13, 1957, pp. 243-250.

305. Segel, R., T. Abbott, Jr., and J. Seehof. High Speed Printer Survey, Part I. National Cash Register Co., Hawthorne. Calif., 15 Jan 1958. 43 p.

306. Setting Type by Electricity, Composition Information Services, Los Angeles, Calif., Reprint from The Inland Printer, Apr 1899, 1 p.

307. Shaw, R. R. Parameters for Machine Handling of Alpha betic Information, Amer. Documentation 1.3, 267-269 (1962).

308. Shepard, F. H., Jr. Method and Apparatus for High Speed Printing. British Patent 825, 646. Patented 16 Dec 1959: filed 13 Aug 1956

309. Shepard 's Citations, Shepard's Citations, Inc., Colorado Springs, Colo., Vol. 1, 1890.

310. Shepard, F. H., Jr. Teleprinter TT-199 (XW-1)/GG. RADC-TR-60-12. Final Rept. on Contract AF 30(602)1792. Shepard Laboratories, Inc., Summit, N.J., 1959, $10 \mathrm{p}$.

311. Shera, J. H., A. Kent and J. W. Perry [eds]. Documentation in Action (Proceedings of Conference on the Practical Utilization of Recorded Knowledge Present and
Future), Reinhold Publishing Corp., New York, 1956, $471 \mathrm{p}$.

312. Shih, B. P. N. and R. L. Snyder. International Únion List of Communist Chinese Serials, M.I.T. Libraries, Cambridıe, Mass., 1963.

313. Shilling, C. W. Requirements for a Scientific MissionOriented Information Center. Amer. Documentation 14. 49-53 (1963).

314. Signs of the Future?, Newsweek 9, 88 (Nov. 1964).

315. Simplified Approach to Mathematical Printing, British Print 74, 137-140 (1961).

316. Skolnick, H. An Experiment in Photocomposition, J. Chem. Doc. 2, i (1962).

317. Smith, F. H. Computers and Filmsetting, Litho-Print 5 9. 11, 12, 15 (Jan. 1962).

318. Smith, F. H. Computers and Filmsetting, Print in Britain 9, 305-307 (1962).

319. Smith, H. O. The Future Import of Photocomposition, Printing Technology 5, 7-22 (Nov. 1961).

320. Smith-Corona Marchant, Inc. Automatic Writing Systems: Tape and Card 1963 Price List, Descriptive Brochure, New York, n.d., 4 p.

321. Sparks. D. E., L. H. Berul and D. P. Waite. Output Printing for Library Mechanization, in B. E. Markuson [ed]. Libraries and Automation, 1964, p. 155-190.

322. Special Computer for Printers, Business Week (7 Mar 1964) p. 102.

323. Spencer, H. The Responsibilities of the Desinn Profession, in H. Spencer [ed], The Penrose Annual, 1964, p. 18-23.

324. Spencer, H. [ed.]. The Penrose Annual, A Review of the Graphic Arts, Hastinøs House, New York, Vol. 57, 1964, $300 \mathrm{p}$.

325. Stevens, M. E. Availability of Machine Usable Natural Language Material, in American University, Machine Indexing: Progress and Problems, 1962, pp. 58-75.

326. Strand Engineering Co. Datronics 200 General Purpose Display System, Descriptive Brochure, Ann Arbor, Mich. 1961, $34 \mathrm{p}$.

327. Swanson, O. D. The Directory Tape Processor, in Re search and Engineering Council of the Craphic Arts Industry, Proceedings of the 13 th Annual Conference 1963, pp. 91-95.

328. Swid, R. E. A Pragmatic Approach to Retrospective Searching, in American Documentation Institute, Param eters of Information Science, 1964, pp. 319-322.

329. Symposium on High-Speed Printing of Computer and Tape Data, in TAGA, Proceedings of the 13th Annual Meeting, 1961.pp. 177-240.

330. TAl;A (Technical Association of the Graphic Arts, Inc.), Proceedings of the 13th Annual Meeting, June 12-14, 1961, Columbus, Ohio, Rochester, N.Y., 1961, 301 p.

331. Taine, S. I. Bibliographic Data Processing at the National Library of Medicine, in H. Goldhor [ed.], Proceedings of the 1963 Clinic on Library Applications of Data Proc essing, 1964, pp. 109-132.

332. Taine, S. I. The Future of the Published Index, in American University, Machine Indexing: Progress and Problems, 1962, pp. 144-169.

333. Tape Operated Linecasting-Developments Merit Renewed Attention, The Typographer 4, 3 (Mar 1962).

334. Tape Processor Speeds Phone Directory Composition, Printing Production (Dec. 1961)

335. Tasman, P. Indexing the Dead Sea Scrolls by Electronic Literary Data Processing Methods. International Business Machines Corp., New York, 1958, 12 p.

336. Technical Announcement Bulletin, Defense Documentation Center, Washington. D.C., No. 65-19, 1 Oct. 1965 $1 \mathrm{v}$

337. Teletypewriter has Capability of 3000 Words per Minute, Aviation Week 69:19 (Nov. 1958).

338. Tholstrup, H. L. Perforated Storage Media, Electrical Manufacturing 62, 53-61 (Dec 1958).

339. Toothman, N. E. The RCA 301 Computer and Typesetting, in Research and Engineering Council of the Graphic Arts Industry, Proceedings of the 13th Annual Conference, 1963, p. 101-108.

340. The Troubled Tide of Automation. Time 86, 45 (16 July 1965). 
341. Typesetting by Computer, Ferranti Computer World, issue of July- Aur. 1964 .

342. U.S. Atomic Eneryy Commission. Terhnical Information Services of the United States Atomic Eneroy Commission, Rept. No. TID-485 (4th Rev.), Division of Technical Information, Washington, D.C., 1962.

343. 1.S. Coneress. House Committee on Science and Astronautics, Dissemination of Scientific Information, Report of the Committee, House Rept. No. 1179, 86th Cong., lst sess., Government Printing Office, Washington, D.C., 1959,12 p.

344. Varitypor Corp. Fotolist Automatic Developer, Descriptive Brochure, Newark, N.J., n.d., 3 p.

345. Varityper Corp. Presenting a New Compact Model Fotolist System Model Ninety, Descriptive Brochure, Newark, N.J., 1962

346. Varityper Corp. The Story of the Fotolist System, Descrip. tive Brochure, Newark, N.J., 1960, 12 p.

347. Varityper Corp. Varityper Series 800 I'hotocomposing Machines, Descriptive Brochure, Newark, N.J., 1963, 7 p.

348. Veilleux, M. I'. P'ermuted Title Work Indexing Procedures for a Man/Machine System, in American University, Machine Indexing: Progress and Problems, 1962, pp. $77-111$.

349. Voigt, V. J. Report on Seriats Computer Project, Iniversity Library and UCSD Computer Center, University of California. San Dieso, La Jolla, Mimeograph, 1962, 32 p.

350. Waite, D. P. Nicrofilm Card is Information Medium for Space Aseney, Systems Manawement (Nov. 1962) p. 22-29.

351. Walden, L. M. Equipment for Type Setting, U.S. Patent 2. 831, 410. Patented 22 Apr 1958; filed 9 Oct 1950 , (Class 95-4.5).

352. Waldo, W. H. Searching Two-Dimensional Structures by Computer, J. Chem. Doc. 2, 1-2 (1962).
353. Washington Enuineering Services Co. Survey of Research on 'Typographical Effectiveness, Summary Rept. to Navy Bureau of Ships on Contract No. 6s 76029 , Bethesda, Md., 1959, 68 p.

354. Webster, E. The Impact of Non-Impact P'rinting, Datamation 9, 24-30 (Sept. 1963).

355. West, R. E. High Speed Readout for Data Processin., Electronics 32, 83-85 (29 May 1959).

356. Winkler, J. H. Research in the (iraphic Arts, in International Association of Printing House Craftsmen, Printine Progress: A Mid-Century Report, 1959. pp. 49-80.

357. Wooster, H. Possible Effects of Current Research in Automatic Information Handlin: on Technical Writing and Publishing, Paper l'resented to the Technical Publishing Society, San Diego, Calif., May 22, 1959.

358. Wyllie, J. C. Science-Technology Subject.Hcading I ists in English, Recent or Still Useful in P'rogramming Information Retrieval Systems, Lniversity of Viruinia Library, Charlottesvilte, Apr. 1965, 8 p.

359. Yasaki, E. The Computer and Newsprint, Datamation 9, 27-31 (Mar. 1963).

360. Youden. W. W. Characteristics of Programs for KWIC and Other Computer-P'roduced Indexes, in H. P'. Luhn [edt.], Automation and Scientific Communiration, Short P'apers, I'ı. 2, 1963, pp. 331-332.

361. Youden, W. W. Computer Literature Bibliosraphy 1946 to 1963, NBS-MP-266, National Bureau of Standards, Waslington, D.C., 31 Mar. 1965, 463 p.

362. Young, C. J., and H. (i. (ireig. Electrofax Direct Electro photographic Printing on Paper, RCA Review 15, 469-484 (1954).

363. Young, D. Faith, Hope, and Computer, Business Automation 6, 20-24 (Dec 1961). 


$$
\begin{aligned}
& 6706029 \\
& 0.471
\end{aligned}
$$

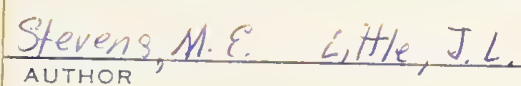

$\frac{\text { Automat ie Typographic -Quality Typesetting }}{\text { TITLE }}$

Techniques: A State of the -Art Review 
U.S. DEPARTMENT OF COMMERCE

WASHINGTON, D.C. 20230
POSTAGE AND FEES PAID

U.S. DEPARTMENT OF COMMERCE

OFFICIAL BUSINESS 